\title{
Open Automated Demand Response Communications Specification (Version 1.0)
}

\author{
Mary Ann Piette, Girish Ghatikar, Sila Kiliccote, Peter Palensky, Charles McParland \\ Lawrence Berkeley National Laboratory
}

Ed Koch, Dan Hennage

Akuacom

The work described in this report was coordinated by the Demand Response Research Center and funded by the California Energy Commission (Energy Commission), Public Interest Energy Research (PIER) Program, under Work for Others Contract No. 500-03-026 and by the U.S. Department of Energy under Contract No. DE-AC02-05CH11231. 


\section{DISCLAIMER}

This report was prepared as the result of work sponsored by the California Energy Commission. It does not necessarily represent the views of the Energy Commission, its employees or the State of California. The Energy Commission, the State of California, its employees, contractors and subcontractors make no warrant, express or implied, and assume no legal liability for the information in this report; nor does any party represent that the uses of this information will not infringe upon privately owned rights. This report has not been approved or disapproved by the California

Energy Commission nor has the California Energy Commission passed upon the accuracy or adequacy of the information in this report.

\section{Disclaimer}

This document was prepared as an account of work sponsored by the United States Government. While this document is believed to contain correct information, neither the United States Government nor any agency thereof, nor The Regents of the University of California, nor any of their employees, makes any warranty, express or implied, or assumes any legal responsibility for the accuracy, completeness, or usefulness of any information, apparatus, product, or process disclosed, or represents that its use would not infringe privately owned rights. Reference herein to any specific commercial product, process, or service by its trade name, trademark, manufacturer, or otherwise, does not necessarily constitute or imply its endorsement, recommendation, or favoring by the United States Government or any agency thereof, or The Regents of the University of California. The views and opinions of authors expressed herein do not necessarily state or reflect those of the United States Government or any agency thereof or The Regents of the University of California.

Ernest Orlando Lawrence Berkeley National Laboratory

Attention: Mary Ann Piette

Demand Response Research Center

One Cyclotron Road, MS 90R3111

Berkeley, CA 94720. United States of America

E-mail: $\underline{\text { AutoDR@lbl.gov }}$

Website: http://openadr.lbl.gov/ 



\section{Acknowledgements}

The work described in this report was coordinated by the Demand Response Research Center and funded by the California Energy Commission (Energy Commission), Public Interest Energy Research (PIER) Program, under Work for Others Contract No. 500-03026 and by the U.S. Department of Energy under Contract No. DE-AC02-05CH11231.

The authors would like to thank the Technical Advisory Group (listed below) for their assistance in this document. We also want to acknowledge Chris Scruton, Ivin Rhyne, Kristy Chew, Martha Brook, and Mike Gravely at the California Energy Commission along with Ron Hofmann and Roger Levy, consultants to the California Energy Commission and LBNL, and David Watson, former LBNL employee, for their ongoing support. We also want to thank Nance Matson for her assistance in finalizing this document. 


\section{Preface}

The California Energy Commission's Public Interest Energy Research (PIER) Program supports public interest energy research and development that will help improve the quality of life in California by bringing environmentally safe, affordable, and reliable energy services and products to the marketplace.

The PIER Program conducts public interest research, development, and demonstration (RD\&D) projects to benefit California.

The PIER Program strives to conduct the most promising public interest energy research by partnering with RD\&D entities, including individuals, businesses, utilities, and public or private research institutions.

- PIER funding efforts are focused on the following RD\&D program areas:

- Buildings End-Use Energy Efficiency

- Energy Innovations Small Grants

- Energy-Related Environmental Research

- Energy Systems Integration

- Environmentally Preferred Advanced Generation

- Industrial/Agricultural/Water End-Use Energy Efficiency

- Renewable Energy Technologies

- Transportation

Open Automated Demand Response Communications Specification is the final report for the Open Automated Demand Response project by the Demand Response Research Center (contract number 500-03-026), conducted by Lawrence Berkeley National Laboratory. The information from this project contributes to PIER's Energy Systems Integration Program.

For more information about the PIER Program, please visit the Energy Commission's Website at www.energy.ca.gov/research/ or contact the Energy Commission at 916-654-4878. 



\section{Table of Contents}

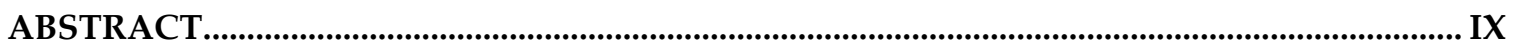

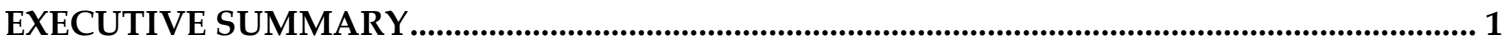

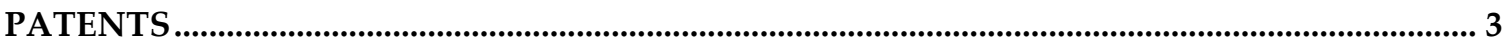

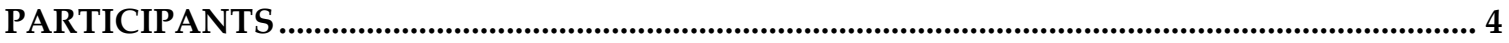

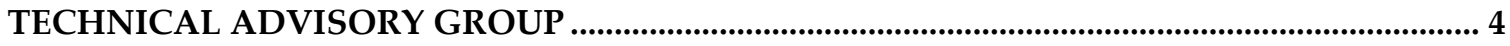

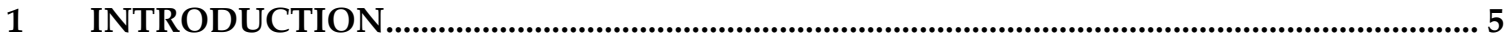

2 SCOPE

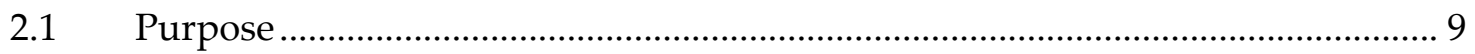

2.2 Reason

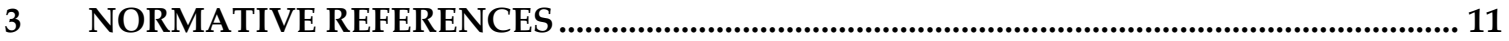

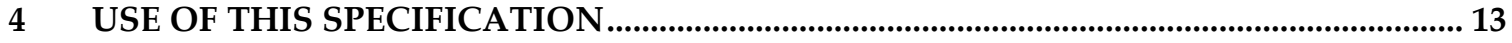

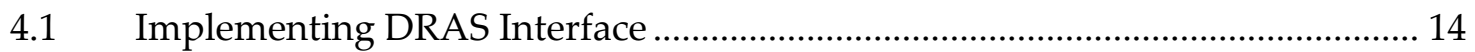

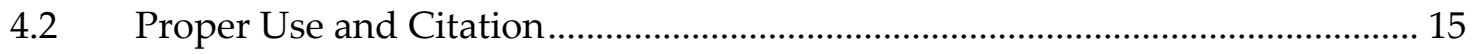

$5 \quad$ DRAS REQUIREMENTS ….................................................................................................... 17

5.1 General Role of DRAS in Demand Response Programs and Dynamic Pricing 17

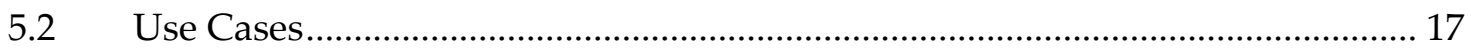

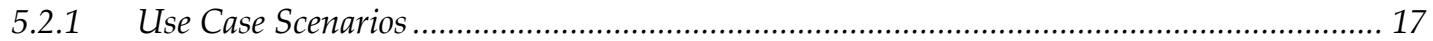

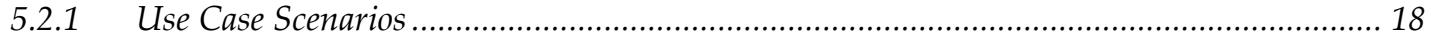

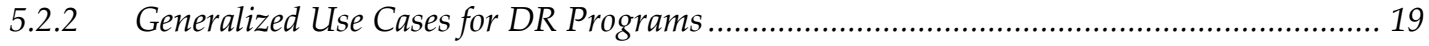

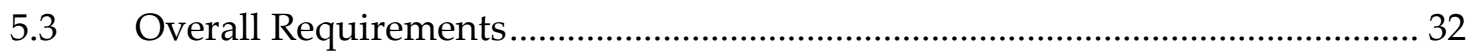

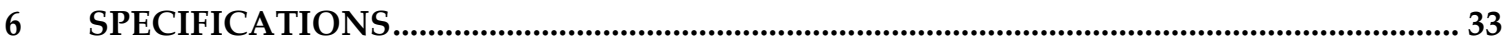

6.1 Automated Demand Response Architecture ….................................................. 33

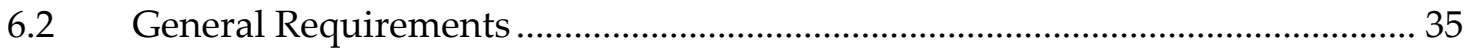

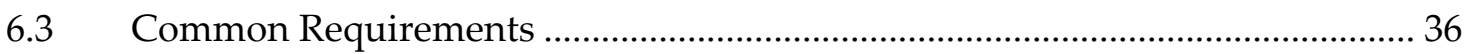

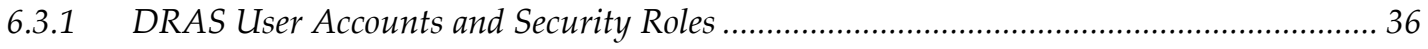

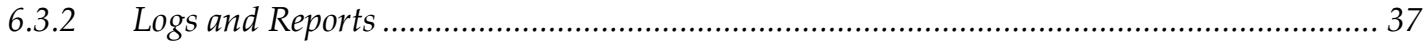

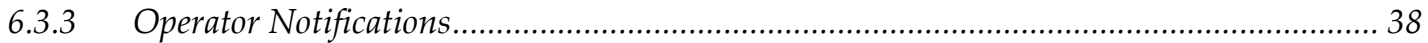

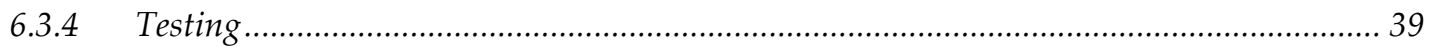

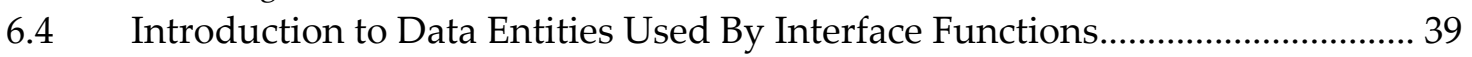

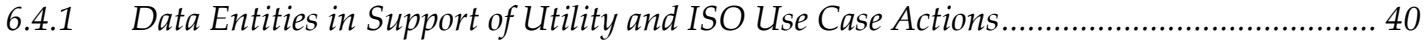

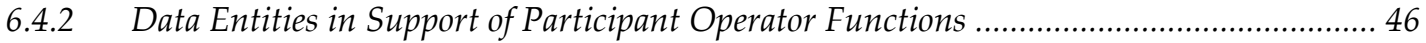

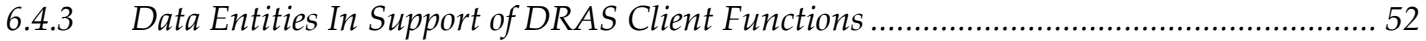

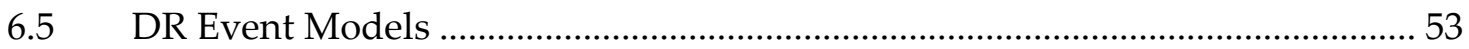

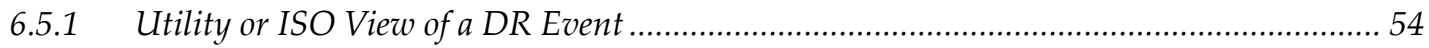




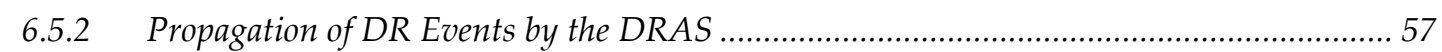

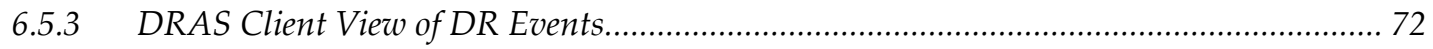

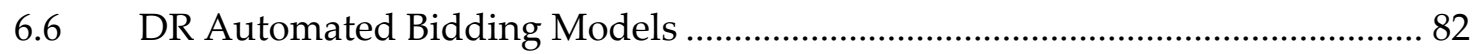

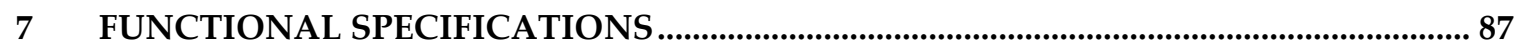

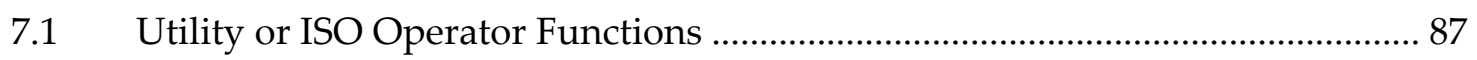

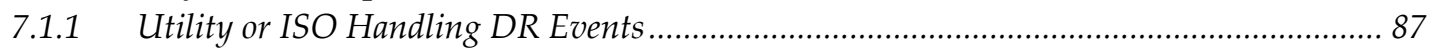

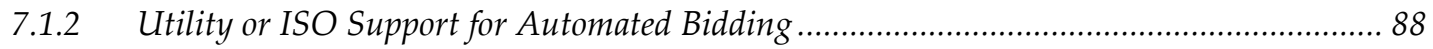

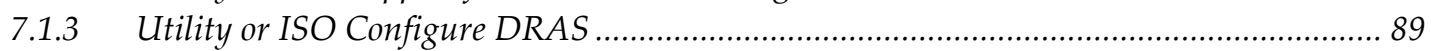

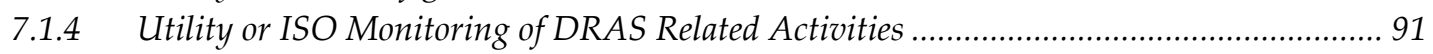

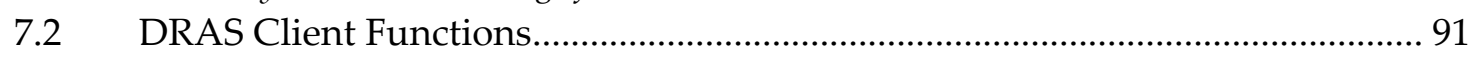

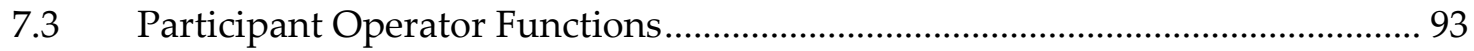

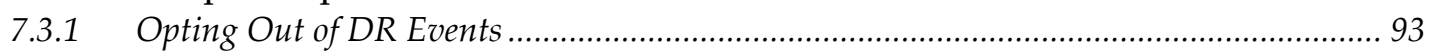

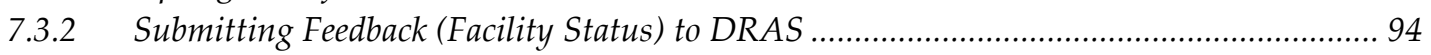

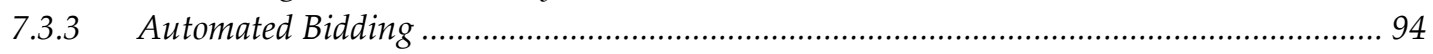

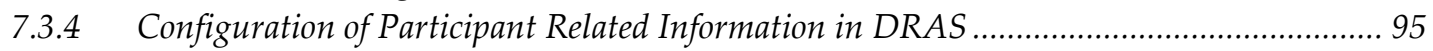

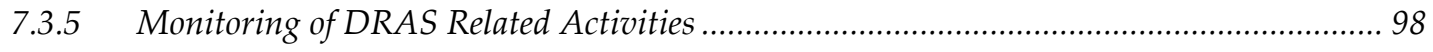

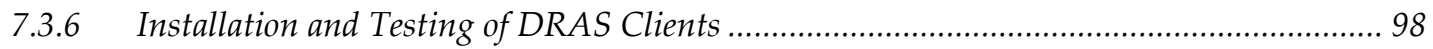

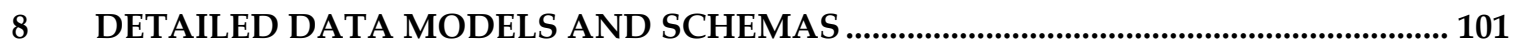

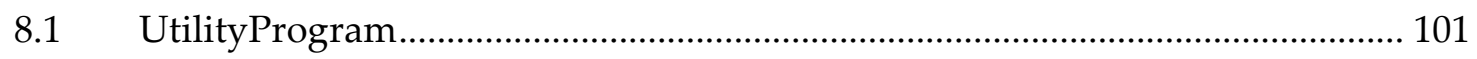

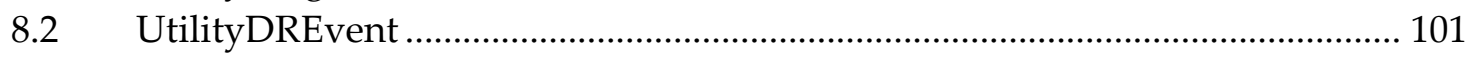

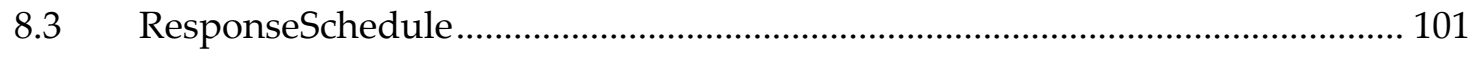

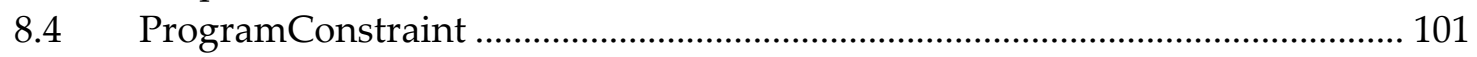

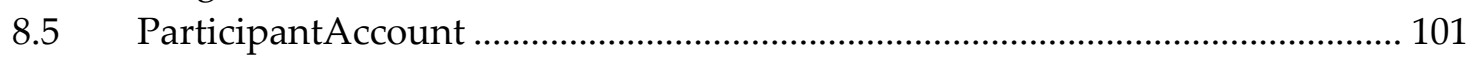

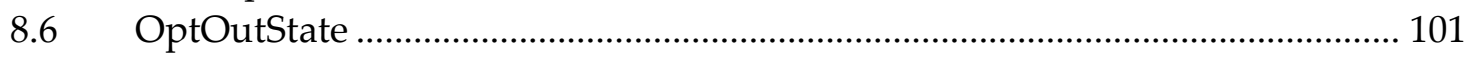

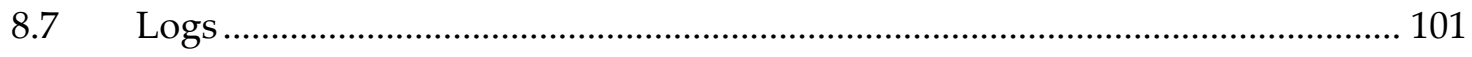

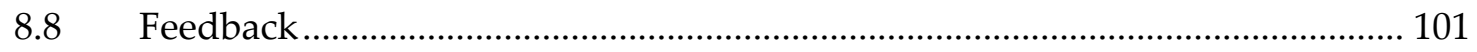

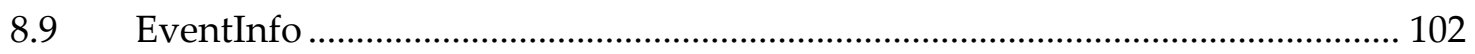

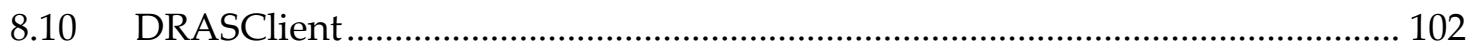

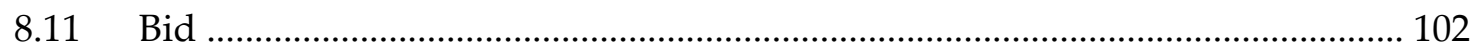

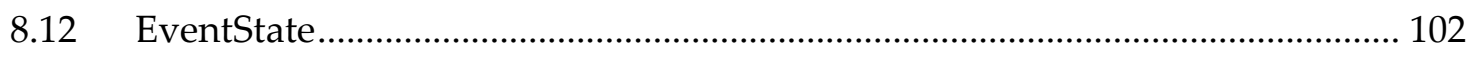

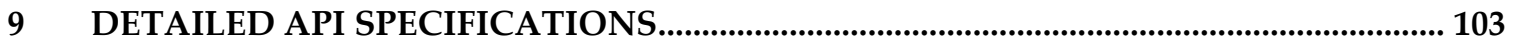

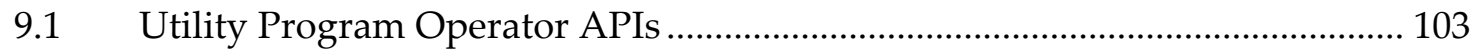

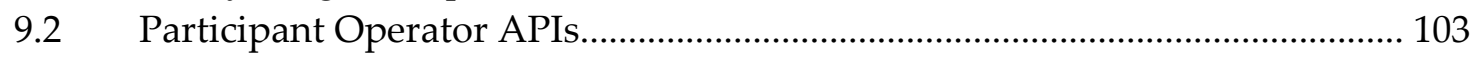

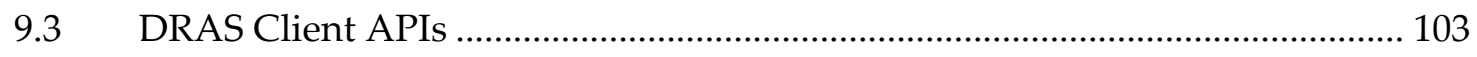

9.3.1 Use of Simple REST Services to Exchange DR EventState Information ........................... 104

9.3.2 Use of Simple SOAP Services to Exchange DR EventState Information ............................ 105

9.3.3 Use of BACnet Web Services to Exchange DR EventState Information............................. 107

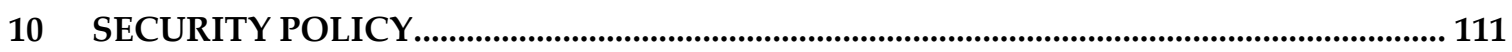

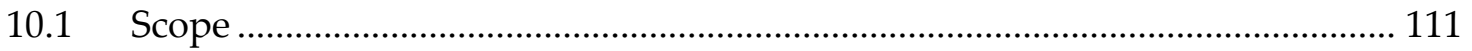

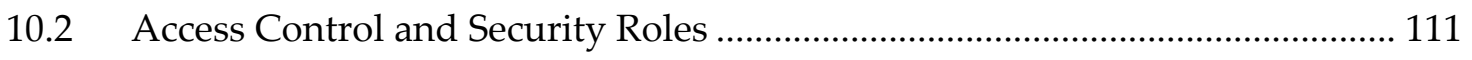

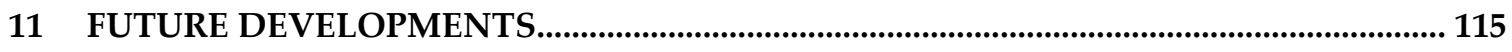


12 DEFINITIONS, ACRONYMS AND ABBREVIATIONS...

APPENDIX A: XSD SCHEMA FILES..

APA-1

APPENDIX B: WSDL INTERFACE FILES ......................................................................................APB-1

APPENDIX C: SECURITY ANALYSIS AND REQUIREMENTS..............................................APC-1

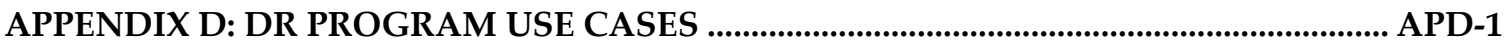




\section{List of Figures}

Figure 1. DRAS Client Interfaces........................................................................... 14

Figure 2. Automated Generic Event-Based Program (GEBP) Use Case............. 24

Figure 3. Generic Bidding Process (GBP) .......................................................... 29

Figure 4. General Automated Events Architecture with Standalone DRAS ..... 34

Figure 5. General Automated Bidding Architecture with Standalone DRAS ... 34

Figure 6. Use Case References to Functional Specification ................................... 35

Figure 7. Utility Issued DR Event Entity …………........................................... 42

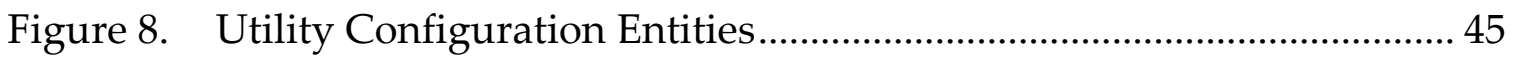

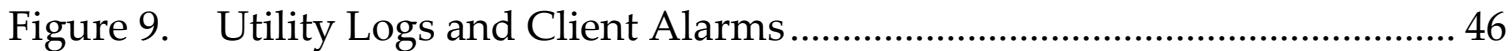

Figure 10. Participant Configuration Entities...................................................... 48

Figure 11. Participant Submit Bid Entity.......................................................... 49

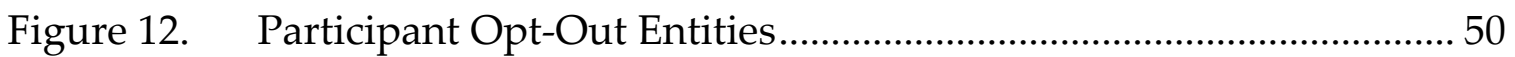

Figure 13. Participant Feedback Entities ............................................................. 51

Figure 14. Client Alarms and Utility Logs ........................................................ 52

Figure 15. DRAS Client DR Event State Entities................................................. 53

Figure 16. DR Event Model (Utility or ISO View) .............................................. 55

Figure 17. State Transition Diagram ................................................................. 55

Figure 18. Relevant Attributes and Structures for Event Propagation .............. 58

Figure 19. Sample Configuration - Participant Accounts and DRAS Clients.. 60

Figure 20. DR Event Propagation for Program P2-All Participant Accounts . 61

Figure 21. DR Event Propagation for Program P2

-Specific Participant Accounts A3, A4

Figure 22. DR Event Propagation for Program P1 .................................................

-Specific Participant Account A1 ........................................................ 63

Figure 23. DR Event Propagation for Program P2-Groups G2, G4 .................. 64

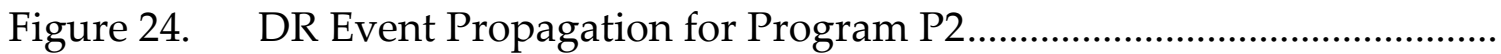

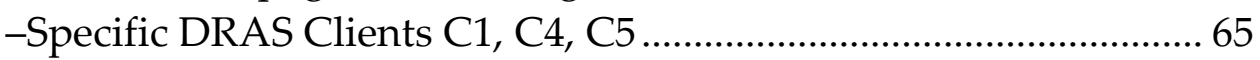

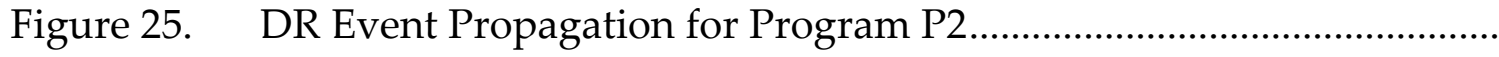

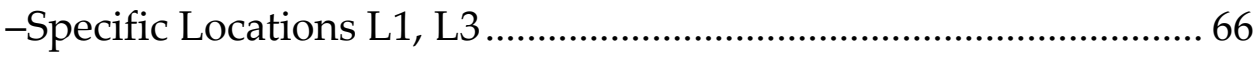

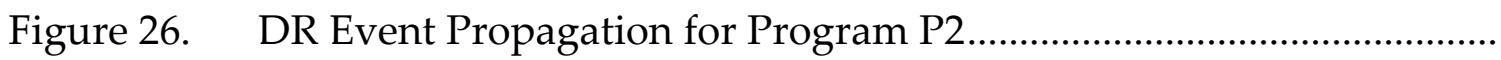

-Groups G2 and Participants A4 Specified ............................................ 67

Figure 27. DR Event Constraint Model

(Which DRAS Clients Receive the Event) ……………...................... 69

Figure 28. Program Constraints and Filters.................................................. 70

Figure 29. DR Event Window Depending Upon Filter Constraints ................. 71

Figure 30. DR Event Notification Time Depending Upon Filter Constraints.. 72 
Figure 31. DR Event Duration Depending Upon Duration Constraints...................... 72

Figure 32. PUSH Model Sequence Diagram...................................................... 74

Figure 33. PULL Model Sequence Diagram ……….......................................... 74

Figure 34. DR Event State Model (Simple Client View) ...................................... 80

Figure 35. DR Event Model (Utility or ISO View) .............................................. 80

Figure 36. Transition Diagram for a General DR Event....................................... 81

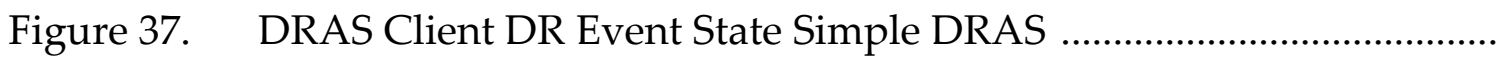

Client State Transition Diagram ......................................................... 82

Figure 38. State Transition Diagram for a Participant's Bid State ..................... 84

Figure 39. Bidding Sequence Diagram ............................................................ 85

Figure 40. REST DRAS Client PULL Interaction Sequence Diagram .............. 104

Figure 41. Simple SOAP PUSH Model Sequence Diagram............................... 106

Figure 42. Simple SOAP PULL Model Sequence Diagram ................................ 106

Figure 43. BWS PUSH Model Sequence Diagram ......................................... 108

Figure 44. BWS PULL Model Sequence Diagram .............................................. 108

Figure 45. DRAS Communication Partners with Different Security Levels.. 114

\section{List of Tables}

Table 1 Relevant Sections of the Specification ………………................................. 13

Table 2 DR Program Execution ............................................................................ 21

Table 3 OperationStateSpec Entity ........................................................................... 79

Table $4 \quad$ Required Services and Supported Communication Models ......................... 104

Table 5 Security Roles of Interfaces .................................................................. 112 


\begin{abstract}
The development of the Open Automated Demand Response Communications Specification, also known as OpenADR or Open Auto-DR, began in 2002 following the California electricity crisis. The work has been carried out by the Demand Response Research Center (DRRC), which is managed by Lawrence Berkeley National Laboratory. This specification describes an open standards-based communications data model designed to facilitate sending and receiving demand response price and reliability signals from a utility or Independent System Operator to electric customers. OpenADR is one element of the Smart Grid information and communications technologies that are being developed to improve optimization between electric supply and demand. The intention of the open automated demand response communications data model is to provide interoperable signals to building and industrial control systems that are preprogrammed to take action based on a demand response signal, enabling a demand response event to be fully automated, with no manual intervention. The OpenADR specification is a flexible infrastructure to facilitate common information exchange between the utility or Independent System Operator and end-use participants. The concept of an open specification is intended to allow anyone to implement the signaling systems, the automation server or the automation clients.
\end{abstract}

Keywords: demand response, buildings, electricity use, automation, communications, open standards, data models, specifications. 


\section{Executive Summary}

The development of the Open Automated Demand Response Communications Specification, also known as OpenADR or Open Auto-DR, began in 2002 following the California electricity crisis. In California, the United States, and abroad many utilities, governments, electric Independent Systems Operators and others have been pursuing demand response to manage the growing demand for electricity and peak capacity of the electric systems. Demand Response (DR) has been defined as “...action taken to reduce electricity demand in response to price, monetary incentives, or utility directives so as to maintain reliable electric service or avoid high electricity prices ${ }^{1}$." OpenADR is one element of the Smart Grid information and communications technologies that are being developed to improve optimization between electric supply and demand.

The research that led to this document was funded by the California Energy Commission's Public Interest Energy Research Program. The work has been carried out by the Demand Response Research Center (DRRC) which is managed by Lawrence Berkeley National Laboratory. The initial goal of the research was to explore the feasibility of developing a low cost communications infrastructure to improve the reliability, repeatability, robustness, and cost-effectiveness of demand response in commercial buildings. One key research question was: could today's communications and information technologies be used to automate demand response operations of commercial buildings using standardized electricity price and reliability signals? Six years of research, development, and demonstration have led to this open data model. This document outlines a communications specification to exchange signals to enable demand response in end-use participant or customer systems.

Open Automated Demand Response is a communications data model designed to facilitate sending and receiving of DR signals from a utility or independent system operator to electric customers. The intention of the data model is to interact with building and industrial control systems that are pre-programmed to take action based on a DR signal, enabling a demand response event to be fully automated, with no manual intervention. The OpenADR specification is a highly flexible infrastructure design to facilitate common information exchange between a utility or Independent System Operator and their end-use participants. The concept of an open specification is intended to allow anyone to implement the signaling systems, the automation server or the automation clients.

\section{Definition of Open Automated Demand Response Communications}

OpenADR Communications have the following defining features:

${ }^{1}$ U.S. Federal Energy Regulatory Commission (FERC), 2007 Assessment of Demand Response and Advanced Metering, Staff Report, available: http://www.ferc.gov/legal/staff-reports/09-07-demandresponse.pdf. 
- Continuous, Secure, and Reliable - Provides continuous, secure, and reliable two-way communications infrastructures where the clients at the end-use site receive and acknowledge to the DR automation sever upon receiving the DR event signals.

- Translation - Translates DR event information to continuous Internet signals to facilitate DR automation. These signals are designed to interoperate with Energy Management and Control Systems, lighting, or other end-use controls.

- Automation - Receipt of the external signal is designed to initiate automation through the use of pre-programmed demand response strategies determined and controlled by the end-use participant.

- Opt-Out - Provides opt-out or override function to participants for a DR event if the event comes at a time when reduction in end-use services is not desirable.

- Complete Data Model - Describes a rich data model and architecture to communicate price, reliability, and other DR activation signals.

- Scalable Architecture - Provides scalable communications architecture to different forms of DR programs, end-use buildings, and dynamic pricing.

- Open Standards - Open standards-based technology such as Simple Object Access Protocol (SOAP) and Web services form the basis of the communications model.

OpenADR has been field tested in a number of DR programs in California. While the scope of this communications specification focuses on signals for DR events and prices, significant research has explored the controls strategies and techniques to automated DR in commercial buildings and industrial facilities. This specification also covers the signaling data model and does not cover information related to specific DR electric reduction or shifting strategies.

The Open Auto-DR Communications Specification is designed to facilitate automating demand response actions at the customer location, whether it is electric load shedding or shifting. We are often asked if the communications data model can be used for continuous operations, every day. The answer is yes. Many emergency or reliability DR events occur at specific times when the electric grid is strained. The Open Auto-DR communications are designed to coordinate such signals to building or industrial control systems. Open Auto-DR is also designed to provide continuous dynamic price signals such as hourly day-ahead or day-of real time pricing. With such price information an automated client can be designed to continuously monitor these prices and translate this information into continuous automated control and response strategies within a facility.

\section{Potential Benefits}

OpenADR will provide benefits to California by both increasing the number of facilities that participate in demand response, and reducing the cost to conduct frequent and persistent participation in demand response. Furthermore OpenADR will improve the 
feasibility of achieving the state's policy goals of moving toward dynamic pricing, such as critical peak or real time pricing, for all customers. Increasing participation in demand response reduces the need for new electric supply, reduces the need for new transmission and distribution systems, and helps reduce overall electricity prices.

The OpenADR Communications Specification provides the following benefits:

- Open Specification-Provides a standardized DR communications and signaling infrastructure using open, non-proprietary, industry-approved data models that can be implemented for both dynamic prices and DR emergency or reliability events.

- Flexibility-Provides open communications interfaces and protocols that are flexible, platform-independent, interoperable, and transparent to end-to-end technologies and software systems.

- Innovation and Interoperability-Encourages open innovation and interoperability, and allows controls and communications within a facility or enterprise to build on existing strategies to reduce technology operation and maintenance costs, stranded assets, and obsolesce in technology.

- Ease of Integration-Facilitates integration of common Energy Management and Control Systems (EMCS), centralized lighting, and other end-use devices that can receive a relay or Internet signals (such aseXtensible Markup Languge [XML]).

- Remote Access- Facilitates opt-out or override functions through a participant Web portal to manage standardized DR-related operation modes to DR strategies and control systems.

\section{Future Research}

The DRRC will continue to conduct research to support broader development and deployment of OpenADR. Future work will include continued collaboration with formal industry standards development organizations to harmonize these data models with related efforts. The DRRC will also continue to evaluate end-use DR control strategies for homes, large and small commercial buildings, and industrial facilities.

\section{Patents}

Attention is called to the possibility that implementation of this specification may require use of subject matter covered by patent rights. By publication of this specification, no position is taken with respect to the existence or validity of any patent rights in connection therewith. The California Energy Commission, Lawrence Berkeley National Laboratory, and the Demand Response Research Center shall not be responsible for identifying patents or patent applications for which a license may be required to implement this specification or for conducting inquiries into the legal validity or scope of those patents that are brought to its attention. 


\section{Participants}

The OpenADR Working Group had the following members:

Ed Koch, Chair, Akuacom

Girish Ghatikar, LBNL Mary Ann Piette, LBNL Dave Robin,

Dan Hennage, Akuacom Peter Palensky,

Sila Kiliccote, LBNL Univ. of Pretoria

Automated Logic

Controls

David Holmberg, NIST

Jim Butler, Cimetrics

Charles McParland, LBNL

\section{Technical Advisory Group}

The following members of the Technical Advisory Group (TAG) members had executive oversight of the OpenADR specification and contributed by review and approval of the specification. Some organizations and affiliations have deputies for continued participation and voting.

\begin{tabular}{|l|l|}
\hline Organization & Member \\
\hline Pacific Gas and Electricity & Peter Chan \\
\hline Pacific Gas and Electricity & Albert Chiu \\
\hline Southern California Edison & Kevin G. Wood \\
\hline Southern California Edison & Eric Parker \\
\hline San Diego Gas and Electric & Terry Mohn \\
\hline San Diego Gas and Electric & Julia Mendoza \\
\hline California Independent Systems Operator & Walt Johnson \\
\hline California Independent Systems Operator & John Goodin \\
\hline National Institute of Standards and Technology & David Holmberg \\
\hline California Energy Commission & Ivin Rhyne \\
\hline Levy Associates & Roger Levy \\
\hline California Institute of Energy and Environment & Ron Hofmann \\
\hline California Institute of Energy and Environment & Gaymond Yee \\
\hline University of California Berkeley & Nate Ota \\
\hline University of California Berkeley & Alex Do \\
\hline Grid Net & David Watson \\
\hline Sacramento Municipal Utility District & Alvaro Mendoza \\
\hline Electric Power Research Institute & Bill Howe \\
\hline Electric Power Research Institute & Chuck Thomas \\
\hline EnerNex Corporation & Eric Gunther \\
\hline EnerNex Corporation & Grant Gilchrist \\
\hline
\end{tabular}




\section{Introduction}

The development of the Open Automated Demand Response Communications Specification, also known as OpenADR or Open Auto-DR, began in 2002 following the California electricity crisis. In California, the United States, and abroad many utilities, governments, electric Independent Systems Operators and others have been pursuing demand response to manage the growing demand for electricity and peak capacity of the electric systems. Demand Response (DR) has been defined as "...action taken to reduce electricity demand in response to price, monetary incentives, or utility directives so as to maintain reliable electric service or avoid high electricity prices ${ }^{1}$." OpenADR is one element of the Smart Grid information and communications technologies that are being developed to improve optimization between electric supply and demand.

The research that led to this document was funded by the California Energy Commission's Public Interest Energy Research Program. The work has been carried out by the Demand Response Research Center (DRRC) which is managed by Lawrence Berkeley National Laboratory. The initial goal of the research was to explore the feasibility of developing a low cost communications infrastructure to improve the reliability, repeatability, robustness, and cost-effectiveness of demand response in commercial buildings. One key research question was: could today's communications and information technologies be used to automate demand response operations of commercial buildings using standardized electricity price and reliability signals? Six years of research, development, and demonstration have led to this open data model. This document outlines a communications specification to exchange signals to enable demand response in end-use participant or customer systems.

Open Automated Demand Response is a communications data model designed to facilitate sending and receiving of DR signals from a utility or independent system operator to electric customers. The intention of the data model is to interact with building and industrial control systems that are pre-programmed to take action based on a DR signal, enabling a demand response event to be fully automated, with no manual intervention. The OpenADR specification is a highly flexible infrastructure design to facilitate common information exchange between a utility or Independent System Operator (ISO) and their end-use participants. The concept of an open specification is intended to allow anyone to implement the signaling systems, providing the automation server or the automation clients.

OpenADR Communications have the following defining features:

- Continuous, Secure, and Reliable - Provides continuous, secure, and reliable twoway communications infrastructures where the clients at the end-use site receive

\footnotetext{
${ }^{1}$ U.S. Federal Energy Regulatory Commission (FERC), 2007 Assessment of Demand Response and Advanced Metering, Staff Report, available: http://www.ferc.gov/legal/staff-reports/09-07-demandresponse.pdf.
} 
and acknowledge to the DR automation sever upon receiving the DR event signals.

- Translation - Translates DR event information to continuous Internet signals to facilitate DR automation. These signals are designed to interoperate with Energy Management and Control Systems, lighting, or other end-use controls.

- Automation - Receipt of the external signal is designed to initiate automation through the use of pre-programmed demand response strategies determined and controlled by the end-use participant.

- Opt-Out - Provides opt-out or override function to participants for a DR event if the event comes at a time when reduction in end-use services is not desirable.

- Complete Data Model - Describes a rich data model and architecture to communicate price, reliability, and other DR activation signals.

- Scalable Architecture - Provides scalable communications architecture to different forms of DR programs, end-use buildings, and dynamic pricing.

- Open Standards - Open standards-based technology such as Simple Object Access Protocol (SOAP) and Web services form the basis of the communications model.

We refer to OpenADR as a "communications data model" to facilitate information exchange between two end-points, the utility or ISO and the facility. It is not a protocol that specifies "bit-structures" or "semantics" as some communications protocols do. In some references the term "system," "technology," or "service" is used to refer to the features of OpenADR.

OpenADR is in use in over 200 facilities in California providing an automation system for several DR programs. These programs provide over $50 \mathrm{MW}$ of DR in commercial and industrial facilities. Several reports present the history of the automated DR research ${ }^{2}$. While the scope of this communications specification focuses on signals for DR events and prices, significant research has explored the controls strategies and techniques to automated DR in commercial buildings ${ }^{3}$. This specification also covers the signaling data model and does not cover information related to specific DR electric

${ }^{2}$ These reports are available at http://drrc.lbl.gov/drrc-pubsall.html:

- Piette, M.A., S. Kiliccote, G. Ghatikar, Design and Implementation of an Open, Interoperable Automated Demand Response Infrastructure, Proceedings of the Grid-Interop Forum, October 2007, LBNL-63665.

- Koch, E., M.A. Piette, Architecture Concepts and Technical Issues for an Open, Interoperable Automated Demand Response Infrastructure. Proceedings of the Grid-Interop Forum,. October 2007. LBNL-63664.

- Piette, M.A, D. Watson, N. Motegi, S. Kiliccote Automated Critical Peak Pricing Field Tests: 2006 Pilot Program Description and Results. August, 2007. LBNL-62218.

${ }^{3}$ Motegi, N., M.A. Piette, D.S. Watson, S. Kiliccote, P. Xu. Introduction to Commercial Building Control Strategies and Techniques for Demand Response. May 2007. LBNL-59975. 
reduction or shifting strategies. This communications specification has also been used for automating DR in industrial facilities.

The Open Auto-DR Communications Specification is designed to facilitate automating demand response actions at the customer location, whether it is electric load shedding or shifting. We are often asked if the communications data model can be used for continuous operations, every day. The answer is yes. Many emergency or reliability DR events occur at specific times when the electric grid is strained. The Open Auto-DR communications are designed to coordinate such signals to building or industrial control systems. Open Auto-DR is also designed to provide continuous dynamic price signals such as hourly day-ahead or day-of real time pricing. With such price information an automated client can be designed to continuously monitor these prices and translate this information into continuous automated control and response strategies within a facility.

The Open Auto-DR Communications Specification provides the following benefits:

- Open Specification-Provides a standardized DR communications and signaling infrastructure using open, non-proprietary, industry-approved data models that can be implemented for both dynamic prices and DR emergency or reliability events.

- Flexibility-Provides open communications interfaces and protocols that are flexible, platform-independent, interoperable, and transparent to end-to-end technologies and software systems.

- Innovation and Interoperability-Encourages open innovation and interoperability, and allows controls and communications within a facility or enterprise to build on existing strategies to reduce technology operation and maintenance costs, stranded assets, and obsolesce in technology.

- Ease of Integration-Facilitates integration of common Energy Management and Control Systems (EMCS), centralized lighting, and other end-use devices that can receive a relay or Internet signals (such as XML).

- Remote Access- Facilitates opt-out or override functions through a participant Web portal to manage standardized DR-related operation modes to DR strategies and control systems.

This report has the following structure. It begins with a section that outlines the scope, purpose, and reason for the OpenADR specification, followed by an introduction to the use of the specification and implementation concepts. Next we present the DR Automation Server (DRAS) requirements, component and functional specifications, followed by the data models and schemas. The final sections discuss the application program interfaces, security policies, and future plans. The future plans for OpenADR include additional collaboration with formal standards groups. A set of appendices contain support schema and interface files, a discussion of security issues, and DR program use cases. 


\section{Scope}

The Open Automated Demand Response Communications Specification defines the interface to the functions and features of a Demand Response Automation Server (DRAS) that is used to facilitate the automation of customer response to various Demand Response programs and dynamic pricing through a communicating client. This specification, referred to as OpenADR, also addresses how third parties such as utilities, ISOs, energy and facility managers, aggregators, and hardware and software manufacturers will interface to and utilize the functions of the DRAS in order to automate various aspects of demand response (DR) programs and dynamic pricing..

\subsection{Purpose}

The success of DR programs and dynamic pricing developed by utilities and ISOs depend upon timely and reliable communications of events and information to the participants that are participating in the DR programs and dynamic pricing. If the DR communications being sent can be automatically translated into load sheds or shifts by the participants without the need for human intervention, then the process of participating in the demand response programs can be made more cost effective, reliable, and easy to implement. This OpenADR specification provides a software interoperability framework, benefit facilities and public or private industry, enable innovation, and ease availability to the widest range of facilities for the present and in the future. This specification describes a suite of functions and capabilities that will allow the automated communications of DR information exchange between utilities or ISOs and their participants. OpenADR is part of the new Smart Grid technologies such as advanced information, control, and communications technologies. These technologies are designed to help optimize the linkages between electric supply and demand.

\subsection{Reason}

Some participants such as aggregators and large corporations have wide spread geographical operations across multiple electrical jurisdictions and thus must deal with multiple utilities. Likewise utilities and ISOs must perform systems integration and testing with each of their participants that participate in a DR program. By using a standardized interchange mechanism like the DRAS across multiple utilities and/or ISOs, the effort and cost of participating in demand response programs and dynamic pricing will be lessened. 


\section{Normative References}

The following referenced documents are useful for the application of this document. For dated references, only the edition cited applies. For undated references, the latest edition of the referenced document (including any amendments or corrigenda) applies. The use of the OpenADR specification does not require Building Automation Control NETwork (BACnet) implementation.

- "BACnet/WS Web Services Interface," ANSI/ASHRAE Addendum Cc to ANSI/ASHRAE Standard 135-2004.

- Request for Comment (RFC): RFC 2246: The Transport Layer Security (TLS) Protocol Version 1.0, Internet Engineering Task Force, Jan 1999. 


\section{Use of this Specification}

This document is designed to specify a set of functions that must be implemented on a so called DRAS. As previously discussed, the DRAS is an infrastructure component that is used for the automated delivery of DR event information to facilities and aggregators. The documentation is intended to satisfy the following:

- Allow utilities and ISOs the ability to interface their Information Technology (IT) infrastructure to a compliant DRAS.

- Allow control manufacturers to interface their EMCS or other controls to a compliant DRAS.

- Allow a variety of operators (e.g. facility and participant operators) to gain an understanding of the level of control in their participation in DR programs and dynamic pricing utilizing a compliant DRAS.

- Allow IT personnel to create user interfaces (UI) for both the utility or ISO and the participant operators of a compliant DRAS.

- Allow third parties to build a compliant DRAS or clients that may receive DR signals from a DRAS or DRAS Client.

This document can be used to satisfy the requirements of a number of entities as described above. It is not necessary that each entity read this document in its entirety. Table 1 gives guidance on the relevant sections of the OpenADR specification that should be read depending upon their requirements.

Table 1 Relevant Sections of the Specification

\begin{tabular}{|l|l|}
\hline Entity & Relevant Sections \\
\hline Utility or ISO interfacing their IT infrastructure to $3^{\text {rd }}$ party DRAS & $0,6,7.1,8,9.1,9.2,10$ \\
\hline $\begin{array}{l}\text { Control manufacturers building equipment to interface to the } \\
\text { DRAS }\end{array}$ & $\begin{array}{l}0,6.1,6.4 .3,6.5 .3,7.2,8.12, \\
9.3,10\end{array}$ \\
\hline Facility and Participant Managers & $0,6,7.3,8,9.2,10$ \\
\hline $\begin{array}{l}\text { Web designers and programmer building Participant user } \\
\text { interfaces. }\end{array}$ & $0,6,7.3,8,9.2,10$ \\
\hline Web designers and programmer building utility user interfaces. & $0,6,7.1,8,9.1,9.2,10$ \\
\hline Implementers of a DRAS & ALL \\
\hline
\end{tabular}




\subsection{Implementing DRAS Interface}

This OpenADR specification is intended to specify the various functions that must exist in a complaint DRAS. It is not intended to specify the precise technology or implementation details of each of the functions in the interface. For example, although this document may specify that SOAP Web services must be used and a Web Service Description Language (WSDL) file may be given for the interface, there is no requirement that a specific language or computing platform be used to actually implement the DRAS. The same is true for many of the data models and entities. While a precise eXtensible Mark-up Language (XML) schema may be given to facilitate the exchange of various pieces of information, there are no requirements on how that information is stored internally or what if any database schemas are used. Also, the look and feel and implementation of the DRAS user interface is outside the scope of this OpenADR specification.

In addition this document describes three distinct types of interfaces which depend upon on what entity is interfacing to the DRAS and include:

- Utility and ISO Operator Interfaces

- Participant Operator Interfaces

- DRAS Client Interfaces

The following diagram, Figure 1, shows the context for these three types of interfaces.

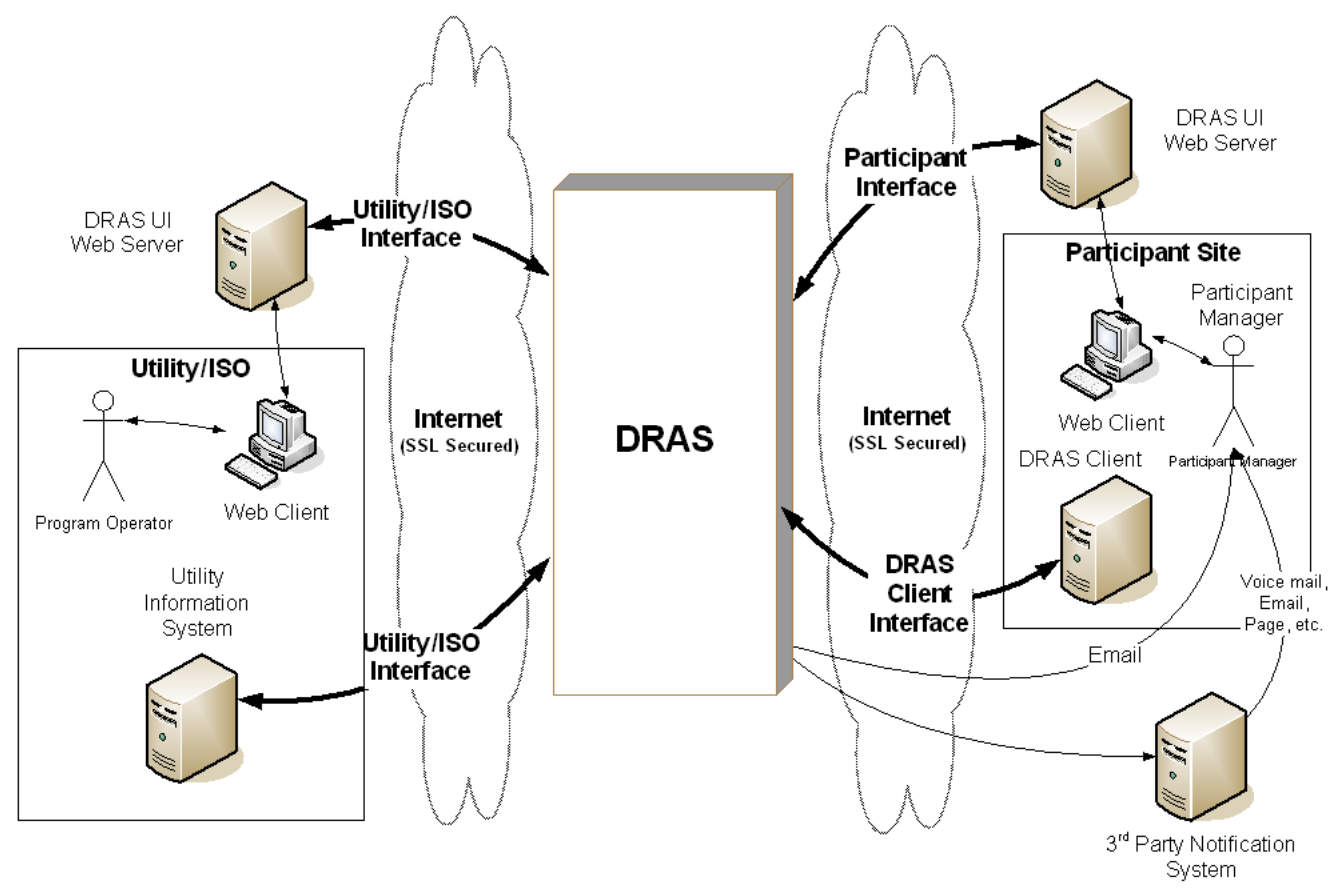

Figure 1. DRAS Client Interfaces 
The purpose of the OpenADR specification is to promote interoperability among various parties. Depending upon how the DRAS is deployed there may not be a requirement for interoperability among one or more of the interfaces described above and thus there may not be a requirement that a DRAS that is compliant with this OpenADR specification implement those interfaces. For example:

- The DRAS is developed by a third party separate from the utility or ISO and the operator interfaces are developed by third party developers. In this case all three interfaces would need to exist for a fully compliant DRAS.

- The DRAS is fully integrated and owned within a utility's IT infrastructure and thus interface group (1) is not necessary. Furthermore the utility developed the Web pages for the various operators and thus interface group (2) is not required. Interface group (3) is still required.

- The DRAS is developed by a third party separate from the utility or ISO, but the operator interfaces were developed by the same party providing the DRAS and have been fully integrated with the DRAS. In this case all interface groups (1) and (3) need to exist, but not (2).

Note that while interface groups (1) and (2) may or may not be required, interface group (3) is always required for a compliant DRAS.

When implementing a DRAS it will be important to specify with which of the interface groups given above the DRAS is compliant. The detailed Application Programming Interface (API) specifications of Section 9 are divided into three sections corresponding to the interface groups given above. In order to be compliant with one of the above sections it will be necessary to implement all of the functions given in the corresponding section of Section 9.

\subsection{Proper Use and Citation}

The proper citation of this OpenADR specification is as follows:

"The (subsystem in question) shall meet or exceed the requirements established in Open Automated Demand Response Communications Specification (Version 1.0)".

Modifications to the OpenADR specification to meet specific circumstances of the user are permissible, so long as they are clearly identified in supporting documentation which accompanies the specification as part of a procurement process. When this is desired, it may be stipulated as in the citation as exampled below:

"The (subsystem in question) must meet the requirements established in Open Automated Demand Response Communications Specification (Version 1.0) for the portion of the OpenADR specification that they are implementing."

Users are strongly discouraged against making generic or unspecific statements such as "(subsystem in question) shall meet all applicable sections of DRRC, Open Automated Demand Response Communications Specification." Such statements create the potential 
for differing assessments by the user and the vendors or supplier as to what is applicable. 


\section{DRAS Requirements}

\subsection{General Role of DRAS in Demand Response Programs and Dynamic Pricing}

The DRAS is an infrastructure component in Automated Demand Response programs that facilitates the communications among the entities (e.g. utilities, ISOs) that produce and distribute electricity and the entities (e.g. facilities and aggregators) that manage the consumption of electricity.

The purpose of the DRAS is to automate the various communication channels necessary for Automated Demand Response programs and dynamic pricing. Such communications include varied price and reliability related messages and information that are sent from utilities or ISOs to the various parties that manage the consumption of electricity in order to curtail the consumption of electricity during peak periods.

\subsection{Use Cases}

This section presents a typical use case of Automated Demand Response programs with the focus being the role of the DRAS in those programs and dynamic pricing. The use case presented in this section is a generalization. Appendix D contains use cases for specific DR programs and dynamic pricing, including detailed descriptions of the symbols and nomenclature used in the use case diagrams. The following roles are used in the use cases.

\subsubsection{Use Case Scenarios}

\subsubsection{Utility Based Roles}

- Utility Program Operator. This is a human operator that manages various aspects of the utility's DR programs and dynamic pricing.

- Program Notifier. This is a computer sub-system or human operator that is responsible for notifying the participants of the DR events and related information.

- Program Settlement. This is a computer sub-system or human operator that is responsible for performing the settlements associated with DR programs and dynamic pricing by measuring the usage of electricity on a per participant basis and feeding the information into the utility's billing system.

\subsubsection{DRAS Roles}

- Event Notifier. This is a sub-system of the DRAS that notifies the participants about DR events initiated by the utility. This is specifically designed for the machine to machine communications necessary to automate the DR program.

- RTP Notifier. This is a sub-system of the DRAS that notifies the participants about real-time pricing (RTP) information as it becomes available. This is 
specifically designed for the machine to machine communications necessary to automate the DR program.

- Program Notifier. This is a sub-system of the DRAS that notifies participant operators of various events related to DR programs and dynamic pricing.

- Bidding Proxy. This is a sub-system of the DRAS that acts as an automated bidding proxy for DR programs and dynamic pricing that require participants to submit bids to the utility.

\subsubsection{DRAS Client Roles}

- DRAS Event Client. This is a sub-system of the DRAS Client and is responsible for notifying the facility's automation sub-systems about DR program events.

- DRAS Feedback Client. This is a sub-system of the DRAS that provides feedback to the DRAS concerning what is happening in a facility in response to a DR event.

- DRAS Operator. A human actor with the responsibility of creating other users.

\subsubsection{Participant Roles}

Participants are the customers of the utilities or ISOs that are participating in the DR programs and dynamic pricing. In general there will be one or more operators as part of the participant's organization that is responsible for managing various aspects of their involvement in the DR program. Within the context of the use cases, there are the following roles:

- Facility Manager. A human operator responsible for managing various aspects of the facility related to the DR program. Within the context of this document a facility manager may also be referred to as a "Participant Manager" or a "Participant Operator".

- Aggregator Manager. A human operator responsible for managing various aspects of the aggregator's participation in the DR program.

\subsubsection{Use Case Scenarios}

Each use case is presented with three broad scenarios:

- Program Configuration

- Program Execution

- Program Maintenance

Each of these scenarios discusses the actions taken by the various roles within that scenario.

\subsubsection{Program Configuration}

Program configuration consists of the actions taken to set up and automate a specific DR program with the emphasis being on the configuration of the DRAS to participate in the program. In some cases, configuration activities may be listed that are not related to the 
DRAS. These activities are listed only to give completeness to the overall DR program and will not be covered in detail.

\subsubsection{Program Execution}

Program execution consists of the actions taken to actually execute and participate in the Auto-DR program. This is the set of actions required for the utility to send DR-related information to the participants that are participating in the program. Emphasis is placed on the actions related to the DRAS. In some cases there may be activities listed those are not related to the DRAS. These activities are listed only to give completeness to the overall DR program and will not be covered in detail.

\subsubsection{Program Maintenance}

Program maintenance consists of the actions related to maintaining a DR program such as changing configurations, generating reports and monitoring DRAS activities.

Emphasis is placed on the actions related to the DRAS. In some cases, activities may be listed that are not related to the DRAS. These activities are listed only to give completeness to the overall DR program and will not be covered in detail.

\subsubsection{Generalized Use Cases for DR Programs}

Table 2 is a spreadsheet that shows the various demand response functions (actions) as they exist across the various use cases documented in Appendix D and is useful for identifying actions which are common across all programs and dynamic pricing. Note that the actions are generalizations of the various actions across all the programs and dynamic pricing listed above.

The following generalizations can be made with respect to the operation of the DRAS:

- The use cases which include the use of aggregators are very similar to the use cases which operate directly with facilities and facility managers; therefore it is reasonable to treat the aggregator roles and the facility manager roles to be equivalent.

- All the use cases that are only for propagating events have very similar sets of steps for the various scenarios. The differences are related to the type of information transmitted with the events.

- All the use cases that automate the bidding process include a very similar set of steps.

- Although the bidding process is linked to specific events there is not a strong coupling between the steps used in the bidding process and the steps involved in propagating events.

In analyzing the DR programs and dynamic pricing so far one can see that there are two general classes of functions:

- Actions related to the automation of DR event notification. 
- Actions related to the automation of the DR bidding process.

Based upon this analysis it is possible to generate general use cases that cover these two functional classes. These use cases are presented in the subsequent sections. 


\section{Table 2 DR Program Execution}

\begin{tabular}{|c|c|c|c|c|c|c|c|c|c|c|c|c|c|c|}
\hline \multirow[b]{3}{*}{ Program } & & \multirow{2}{*}{\multicolumn{3}{|c|}{ Configuration }} & \multicolumn{8}{|c|}{ DR Program Execution } & & \\
\hline & & & & & \multicolumn{5}{|c|}{ Actions on DRAS } & \multicolumn{3}{|c|}{ Actions By DRAS } & \multicolumn{2}{|c|}{ Maintenance } \\
\hline & & $\begin{array}{c}\text { Configure } \\
\text { Program }\end{array}$ & $\begin{array}{l}\text { Configure } \\
\text { DRAS Client } \\
\text { Connection }\end{array}$ & $\begin{array}{c}\text { Configure } \\
\text { Bids }\end{array}$ & $\begin{array}{l}\text { Request } \\
\text { Bids }\end{array}$ & $\begin{array}{l}\text { Initiate } \\
\text { DR } \\
\text { Event }\end{array}$ & $\begin{array}{l}\text { Program } \\
\text { Opt Out }\end{array}$ & $\begin{array}{l}\text { Set } \\
\text { Load } \\
\text { Status }\end{array}$ & $\begin{array}{l}\text { Set } \\
\text { Current } \\
\text { Bids }\end{array}$ & $\begin{array}{c}\text { Send } \\
\text { DR } \\
\text { Event } \\
\text { Info }\end{array}$ & $\begin{array}{l}\text { Notify } \\
\text { Request } \\
\text { for Bid }\end{array}$ & $\begin{array}{l}\text { Notify } \\
\text { Bid } \\
\text { Status }\end{array}$ & $\begin{array}{l}\text { Utility } \\
\text { Operator } \\
\text { Reports }\end{array}$ & $\begin{array}{c}\text { Client } \\
\text { Reports }\end{array}$ \\
\hline \multirow{5}{*}{ CPP } & $\begin{array}{l}\text { Utility } \\
\text { Operator }\end{array}$ & $x$ & & & & & & & & & & & $x$ & \\
\hline & $\begin{array}{l}\text { Utility } \\
\text { Program } \\
\text { Notifier }\end{array}$ & & & & & $x$ & & & & & & & & \\
\hline & $\begin{array}{l}\text { Utility Info } \\
\text { System }\end{array}$ & & & & & & & & & & & & & \\
\hline & DRAS Client & & & & & & & $x$ & & $x$ & & & & \\
\hline & $\begin{array}{l}\text { Client } \\
\text { Operator }\end{array}$ & & $x$ & & & & $x$ & & & & & & & $\mathrm{X}$ \\
\hline \multirow{5}{*}{ DBP } & $\begin{array}{l}\text { Utility } \\
\text { Operator }\end{array}$ & $x$ & & & & & & & & & & & $x$ & \\
\hline & $\begin{array}{l}\text { Utility } \\
\text { Program } \\
\text { Notifier } \\
\end{array}$ & & & & $x$ & $x$ & & & & & & & & \\
\hline & $\begin{array}{l}\text { Utility Info } \\
\text { System }\end{array}$ & & & & & & & & $x$ & & & & & \\
\hline & DRAS Client & & & & & & & $x$ & & $x$ & & & & \\
\hline & $\begin{array}{l}\text { Client } \\
\text { Operator }\end{array}$ & & $x$ & $\mathrm{x}$ & & & $\mathrm{x}$ & & & & $\mathrm{x}$ & $x$ & & $\mathrm{x}$ \\
\hline \multirow{5}{*}{ CBP } & $\begin{array}{l}\text { Utility } \\
\text { Operator }\end{array}$ & $x$ & & & & & & & & & & & $x$ & \\
\hline & $\begin{array}{l}\text { Utility } \\
\text { Program } \\
\text { Notifier }\end{array}$ & & & & $x$ & $x$ & & & & & & & & \\
\hline & $\begin{array}{l}\text { Utility Info } \\
\text { System }\end{array}$ & & & & & & & & $x$ & & & & & \\
\hline & DRAS Client & & & & & & & $x$ & & $x$ & & & & \\
\hline & $\begin{array}{l}\text { Client } \\
\text { Operator }\end{array}$ & & $\mathrm{x}$ & $x$ & & & $x$ & & & & $x$ & $x$ & & $x$ \\
\hline
\end{tabular}




\begin{tabular}{|c|c|c|c|c|c|c|c|c|c|c|c|c|c|c|}
\hline \multirow[b]{3}{*}{ Program } & & \multirow{2}{*}{\multicolumn{3}{|c|}{ Configuration }} & \multicolumn{8}{|c|}{ DR Program Execution } & & \\
\hline & & & & & \multicolumn{5}{|c|}{ Actions on DRAS } & \multicolumn{3}{|c|}{ Actions By DRAS } & \multicolumn{2}{|c|}{ Maintenance } \\
\hline & & $\begin{array}{l}\text { Configure } \\
\text { Program }\end{array}$ & $\begin{array}{l}\text { Configure } \\
\text { DRAS Client } \\
\text { Connection }\end{array}$ & $\begin{array}{c}\text { Configure } \\
\text { Bids }\end{array}$ & $\begin{array}{l}\text { Request } \\
\text { Bids }\end{array}$ & $\begin{array}{l}\text { Initiate } \\
\text { DR } \\
\text { Event }\end{array}$ & $\begin{array}{l}\text { Program } \\
\text { Opt Out }\end{array}$ & $\begin{array}{l}\text { Set } \\
\text { Load } \\
\text { Status }\end{array}$ & $\begin{array}{l}\text { Set } \\
\text { Current } \\
\text { Bids }\end{array}$ & $\begin{array}{c}\text { Send } \\
\text { DR } \\
\text { Event } \\
\text { Info }\end{array}$ & $\begin{array}{l}\text { Notify } \\
\text { Request } \\
\text { for Bid }\end{array}$ & $\begin{array}{l}\text { Notify } \\
\text { Bid } \\
\text { Status }\end{array}$ & $\begin{array}{l}\text { Utility } \\
\text { Operator } \\
\text { Reports }\end{array}$ & $\begin{array}{c}\text { Client } \\
\text { Reports }\end{array}$ \\
\hline \multirow{5}{*}{ BIP } & $\begin{array}{l}\text { Utility } \\
\text { Operator }\end{array}$ & $x$ & & & & & & & & & & & $x$ & \\
\hline & $\begin{array}{l}\text { Utility } \\
\text { Program } \\
\text { Notifier }\end{array}$ & & & & & $x$ & & & & & & & & \\
\hline & $\begin{array}{l}\text { Utility Info } \\
\text { System }\end{array}$ & & & & & & & & & & & & & \\
\hline & $\begin{array}{l}\text { DRAS } \\
\text { Client }\end{array}$ & & & & & & & $\mathrm{x}$ & & $\mathrm{X}$ & & & & \\
\hline & $\begin{array}{l}\text { Client } \\
\text { Operator }\end{array}$ & & $x$ & & & & $x$ & & & & & & & $\mathrm{x}$ \\
\hline \multirow{5}{*}{ PDC } & $\begin{array}{l}\text { Utility } \\
\text { Operator }\end{array}$ & $\mathrm{X}$ & & & & & & & & & & & $\mathrm{x}$ & \\
\hline & $\begin{array}{l}\text { Utility } \\
\text { Program } \\
\text { Notifier }\end{array}$ & & & & & $\mathrm{x}$ & & & & & & & & \\
\hline & $\begin{array}{l}\text { Utility Info } \\
\text { System }\end{array}$ & & & & & & & & & & & & & \\
\hline & $\begin{array}{l}\text { DRAS } \\
\text { Client }\end{array}$ & & & & & & & $x$ & & $\mathrm{x}$ & & & & \\
\hline & $\begin{array}{l}\text { Client } \\
\text { Operator }\end{array}$ & & $x$ & & & & $\mathrm{X}$ & & & & & & & $\mathrm{x}$ \\
\hline \multirow{5}{*}{ РCT } & $\begin{array}{l}\text { Utility } \\
\text { Operator }\end{array}$ & $x$ & & & & & & & & & & & $\mathrm{x}$ & \\
\hline & $\begin{array}{l}\text { Utility } \\
\text { Program } \\
\text { Notifier }\end{array}$ & & & & & $x$ & & & & & & & & \\
\hline & $\begin{array}{l}\text { Utility Info } \\
\text { System }\end{array}$ & & & & & & & & & & & & & \\
\hline & $\begin{array}{l}\text { DRAS } \\
\text { Client }\end{array}$ & & & & & & & $x$ & & $x$ & & & & \\
\hline & $\begin{array}{l}\text { Client } \\
\text { Operator }\end{array}$ & & $\mathrm{x}$ & & & & $\mathrm{X}$ & & & & & & & $\mathrm{x}$ \\
\hline
\end{tabular}




\begin{tabular}{|c|c|c|c|c|c|c|c|c|c|c|c|c|c|c|}
\hline \multirow[b]{3}{*}{ Program } & & \multirow{2}{*}{\multicolumn{3}{|c|}{ Configuration }} & \multicolumn{8}{|c|}{ DR Program Execution } & & \\
\hline & & & & & \multicolumn{5}{|c|}{ Actions on DRAS } & \multicolumn{3}{|c|}{ Actions By DRAS } & \multicolumn{2}{|c|}{ Maintenance } \\
\hline & & $\begin{array}{l}\text { Configure } \\
\text { Program }\end{array}$ & $\begin{array}{c}\text { Configure } \\
\text { DRAS Client } \\
\text { Connection }\end{array}$ & $\begin{array}{c}\text { Configure } \\
\text { Bids }\end{array}$ & $\begin{array}{c}\text { Request } \\
\text { Bids }\end{array}$ & $\begin{array}{c}\text { Initiate } \\
\text { DR } \\
\text { Event } \\
\end{array}$ & $\begin{array}{l}\text { Program } \\
\text { Opt Out }\end{array}$ & $\begin{array}{l}\text { Set } \\
\text { Load } \\
\text { Status }\end{array}$ & $\begin{array}{c}\text { Set } \\
\text { Current } \\
\text { Bids }\end{array}$ & $\begin{array}{c}\text { Send } \\
\text { DR } \\
\text { Event } \\
\text { Info }\end{array}$ & $\begin{array}{l}\text { Notify } \\
\text { Request } \\
\text { for Bid }\end{array}$ & $\begin{array}{l}\text { Notify } \\
\text { Bid } \\
\text { Status }\end{array}$ & $\begin{array}{l}\text { Utility } \\
\text { Operator } \\
\text { Reports }\end{array}$ & $\begin{array}{l}\text { Client } \\
\text { Reports }\end{array}$ \\
\hline \multirow{5}{*}{ RTP } & $\begin{array}{l}\text { Utility } \\
\text { Operator }\end{array}$ & $x$ & & & & & & & & & & & $x$ & \\
\hline & $\begin{array}{l}\text { Utility } \\
\text { Program } \\
\text { Notifier }\end{array}$ & & & & & $\mathrm{x}$ & & & & & & & & \\
\hline & $\begin{array}{l}\text { Utility Info } \\
\text { System }\end{array}$ & & & & & & & & & & & & & \\
\hline & $\begin{array}{l}\text { DRAS } \\
\text { Client }\end{array}$ & & & & & & & $\mathrm{x}$ & & $\mathrm{x}$ & & & & \\
\hline & $\begin{array}{l}\text { Client } \\
\text { Operator }\end{array}$ & & $\mathrm{x}$ & & & & $\mathrm{x}$ & & & & & & & $\mathrm{x}$ \\
\hline
\end{tabular}




\subsubsection{Generic Event-Based Programs (GEBP)}

As stated, there are many similarities in the sequence of steps used to propagate events in the various use cases. The use case presented in this section represents a generalization of those use cases into a Generic Event Based Program (GEBP) (Figure 2). which is based on the General (GEN) use case diagram. Note that the following generalizations have been made:

- There is no distinction between the various types of participants that interface to the DRAS or participate in the program.
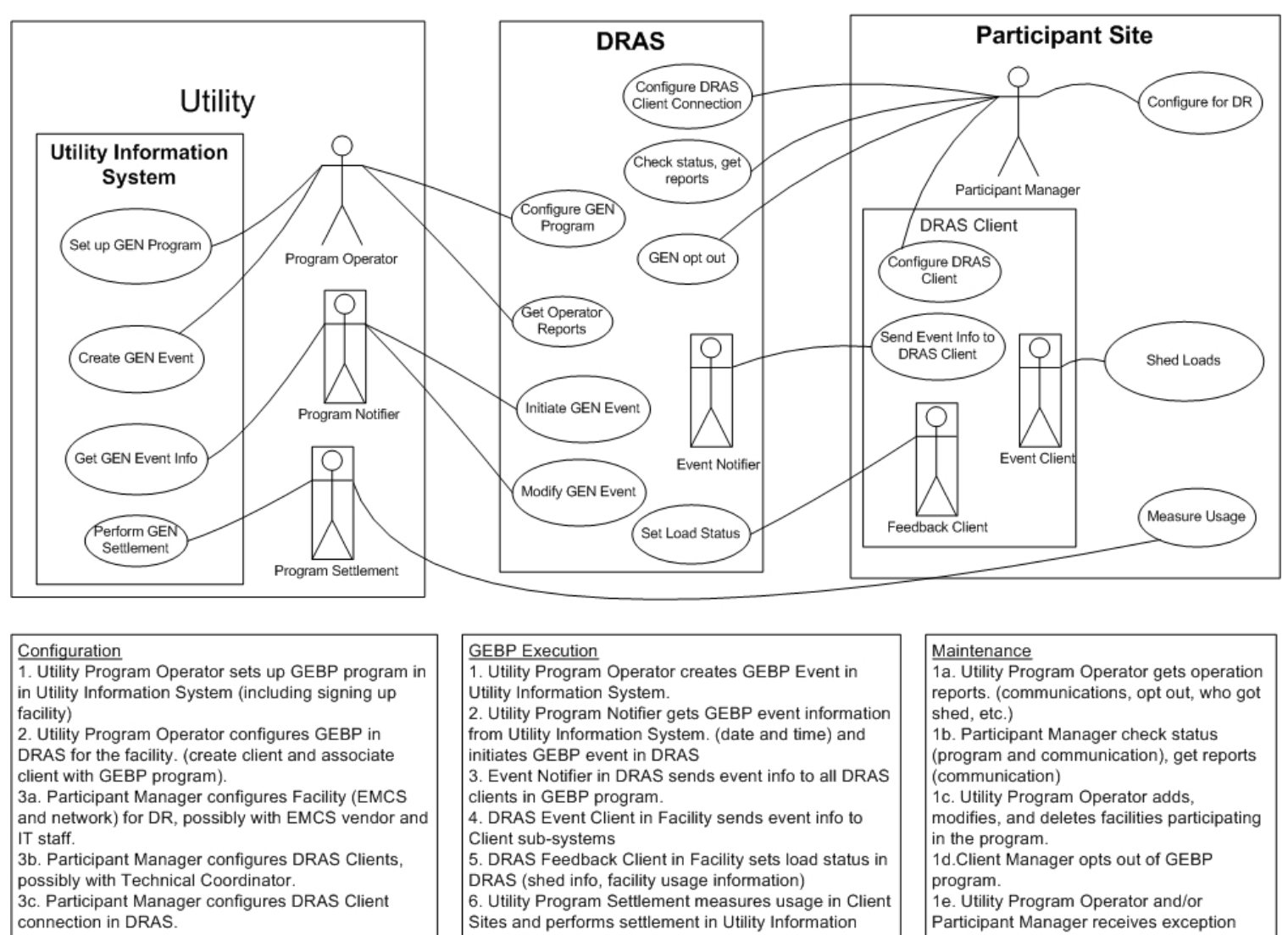

3b. Participant Manager configures DRAS Clients, possibly with Technical Coordinator.

3c. Participant Manager configures DRAS Client connection in DRAS.

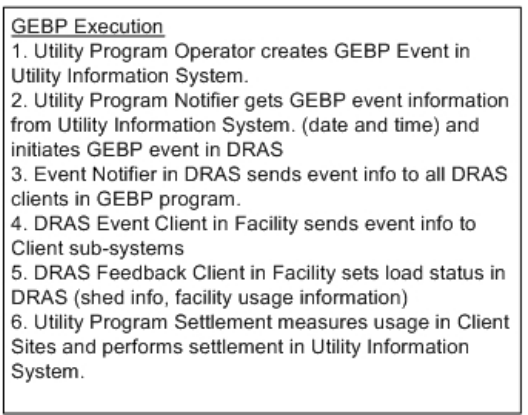

Maintenance

1a. Utility Program Operator gets operation reports. (communications, opt out, who got shed, etc.)

1b. Participant Manager check status (program and communication), get reports (communication)

1c. Utility Program Operator adds, modifies, and deletes facilities participating in the program.

1d.Client Manager opts out of GEBP program.

program.
1e. Utility Program Operator and/or Participant Manager receives exception notification from DRAS in case of error. (Not shown in diagram)

Figure 2. Automated Generic Event-Based Program (GEBP) Use Case

\subsection{GEBP Configuration}

This includes entering all the information necessary for the participant to participate in the GEBP DR program and involves the following actions:

1. The utility program operator sets up the GEBP program in their Utility Information System (UIS). This includes signing up participants and entering all required information necessary for the participants to participate in the GEBP program into the UIS. The details of this process are beyond the scope of this document. 
2. The utility program operator configures the GEBP in the DRAS for the facility. This includes entering information into the DRAS to allow the participant manager to access the DRAS and set up their DRAS Client so that it may communicate with the DRAS. It includes entering the following information:

- Program definition:

o Program schedule constraints such as time of day, duration, etc.

o Type of information (e.g. prices, levels, etc.) specified as part of a DR event.

o Program event information provided to DRAS Client for signal mapping

- Utility assigned account number used for settlement

- Participant identification

- Participant password

- Geographic location

- Grid location

3a. The participant manager configures the participant site's EMCS or network for DR, possibly in conjunction with the EMCS vendor and IT staff. This could include programming the EMCS to respond to DR events and shed or shift loads appropriately. The details of this are beyond the scope of this document.

3b. The participant manager configures the DRAS Clients. DRAS Clients may take many forms, both in terms of hardware and software. This step configures the DRAS Client so that it can communicate with the facilities' systems that are responsible for managing the loads. The details of the process are beyond the scope of this document.

3c. The participant manager configures the DR program parameters and DRAS Client connection in DRAS. This step establishes the connection between the DRAS Client and the DRAS. Typically this includes the following types of information:

- Identification and password of the participant

- Contact information (phone number, pager, email address, etc.)

- DRAS Client communications parameters

- DRAS IP address

o Identification

o Password

o IP connection information

o Polling frequency (if DRAS Client is polling)

- Optional - Load reduction potential (per time block per level)

- Exception parameters 
- Opt-out dates

Note that this action is currently depicted as occurring in the DRAS, but depending upon how this step is subsequently implemented in a future formal OpenADR standard; this may be performed in the DRAS Client and not in the DRAS.

\subsection{GEBP Execution}

The set of actions to execute GEBP events include the following steps:

1. The utility program operator creates the GEBP DR event in the Utility Information System. In this step, a program operator schedules a GEBP event in the Utility Information System. The details of this is process are beyond the scope of this document.

2. The utility program notifier gets the GEBP DR event information from their Utility Information System and initiates the GEBP DR event in the DRAS. The information sent to the DRAS by the utility program notifier sub-system includes the following information:

- Program type

- Date and time of the event

- Date and time issued

- Geographic location

- Participants list (account numbers)

3. The event notifier in the DRAS sends the GEBP DR event information to the appropriate DRAS Clients in the GEBP DR program. The GEBP DR event information sent to the DRAS Clients includes the following:

- Utility event information for intelligent DRAS Clients

o Date and time of the event

o Date and time issued

o Geographic location (optional)

o Mode and pending signals

- Mode signal levels for simple DRAS Clients (e.g. normal, moderate, high)

- Event pending signal for simple DRAS Clients (e.g. yes/no, or simple quantification of how far in advance notification is to be sent)

As part of this interaction, there is a confirmation message sent by the DRAS Client to the DRAS to indicate that it has received the DR event information. In addition there is an indicator of whether a DRAS Client is opting in or out of a DR event when it sends the confirmation message. This allows the opt-out function to be performed on the automation equipment in the facility and sent to the DRAS as part of the DRAS and DRAS Client interaction. 
4. The DRAS event client at the participant site sends the event information to the participant systems responsible for load shed. The details of this process are beyond the scope of this document.

5. The DRAS feedback client at the participant site sends the system load status to the DRAS. This is a feedback mechanism that is used to record how the participant site responded to the DR event or status of the facility outside an event (e.g. near real time load). It includes the following information:

- Program identifier

- Facility identifier

- Date and time of the event (shed or shift)

- Shed data in $\mathrm{kW} / \mathrm{kWh}$

- Near real time load

- Load reduction end uses (Heating, ventilation and air conditioning [HVAC], lighting)

- $\quad$ Event Type (Day-ahead, Day-of)

6. The utility program settlement program measures usage in a facility and conducts the settlement activity in the Utility Information System. This process is beyond the scope of this document.

Note that one of the functions not included in the steps given above is the ability for the utility program operator to modify or cancel an already issued DR event. This is shown in Figure 2 as "Modify GEN Event."

\subsection{GEBP Maintenance}

This scenario consists of a set of actions which are necessary to maintain the GEBP program. Unlike the configuration and execution scenarios that have a prescribed set of steps, this set of actions are the possible actions that may be performed by the various roles at unrelated times.

1a. The utility program operator gets the operation reports. The participant manager can get the following status information from the DRAS at any time:

- Event status (for all participants):

o current outstanding events

- Load reduction potential based upon all participants in program (optional)

- Feedback from DRAS Clients (for those that have optional feedback)

o Event logs

1b. The participant manager checks the status. The utility program operator can get the following status information from the DRAS at any time: 
- DRAS Client communications status:

o Current status

o Last contact

o Current signals levels

o Current participant manual control levels (opt-out)

o Communication logs

o Signal logs

o Manual control logs

- Event status (same as above)

1c. The utility program operator adds, modifies, and deletes facilities participating in the program. This is similar to the original configuration step.

$1 \mathrm{~d}$. The participant manager opts out of the DR program. At any time, the participant manager can opt out of the DR program on the DRAS. When in an opt-out condition, DR events are not propagated to the DRAS Client. The opt-out can be either for the entire program or a single event. There is a method as part of the participant interface to allow operators to opt out of a DR event at any time on the DRAS.

1e. The utility program operator and/or the participant manager receive an exception notification from the DRAS when there is an error (this is not shown in the diagram). When an error condition occurs in the DRAS which may require some sort of action by either the participant manager or the utility program operator, the DRAS will send a message to the respective operators via email, voice or pager. Note that this alarming interface does not cover exception conditions that are part of the DRAS operation and managed by the DRAS operator. Such exceptions might include machine and platform specific exceptions such as "out of disk space" and are outside the scope of this document. The types of exceptions covered by this interface include DRAS Client communications failure.

\subsubsection{Generic Bidding Programs (GBP)}

This section presents a use case for Generic Bidding Programs (GPB, Figure 3). This is intended to represent how a generalized bidding process may be automated by the DRAS. This covers only the bidding and bid acceptance process and does not cover the event propagation process which was presented in the previous section. 


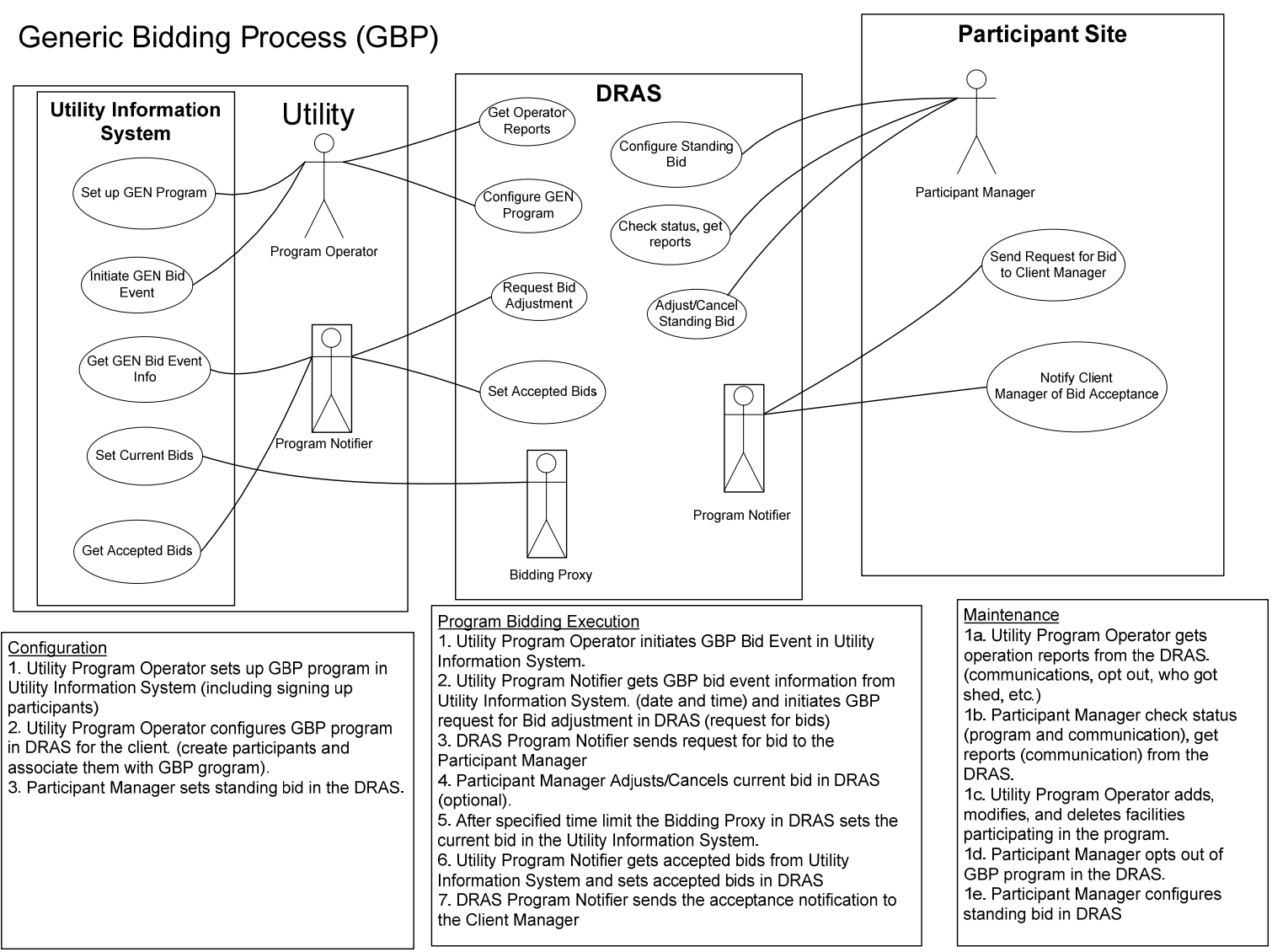

Figure 3. Generic Bidding Process (GBP)

\subsection{GBP Configuration}

This includes entering all the information necessary for the participant to participate in the DR program and involves the following actions.

1. The utility program operator sets up the GBP program in the Utility Information System. This includes signing up participants and entering all required information necessary for the participant to participate in the GBP program into the UIS. The details of this process are beyond the scope of this document.

2. The utility program operator configures the GBP in the DRAS for the facility. This includes entering information into the DRAS to allow the facility operator to access the DRAS and set up their DRAS Client so that it may communicate with the DRAS. It includes entering the following information:

- Program definition:

o Program schedule constraints such as time of day, duration, etc.

o Type of information (e.g. prices, levels, etc.) specified as part of a DR event.

o Program event information to DRAS Client signal mapping

- Utility assigned account number used for settlement 
- Participant identification

- Participant password

- Geographic location

- Grid location

- Manager contact information (email address, telephone, pager number, etc.)

3. The participant manager sets standing bid in the DRAS. This is the bid that will be automatically placed by the DRAS when a request for bid comes from the utility. It includes the following:

- Load reduction bids per time block (price and load amount)

\subsection{GBP Execution}

The bidding process includes the following steps:

1. The utility program operator creates a GBP bidding event in Utility Information System. In this step, a program operator schedules a GBP bidding event in the Utility Information System. The details of this process are beyond the scope of this document.

2. The utility program notifier gets GBP bidding event information from the Utility Information System and initiates GBP Request for bids in the DRAS. The information sent to the DRAS by the utility program notifier sub-system includes the following information:

- Program type

- Date and time of the event

- Date and time issued

- Geographic location

- Participant list (account numbers)

- Request For Bids (RFB) issue date and time

- $\quad$ RFB close time

- Price offered for load reduction per time block

3. The DRAS program notifier sends request for bid to the participant manager. This notification typically comes in the form of an email, phone call or page.

4. The participant manager can adjust or cancel the current bid in the DRAS. This is an optional step and allows the manager to adjust their bid for that particular event. If this step is not performed then the DRAS will submit the standing bid after the end of the bid period.

5. After the bidding time limit has expired, the bidding proxy in the DRAS sets the current bid in the Utility Information System. The information sent by the DRAS includes the following for each participant:

- Participant account number 
- Load reduction bids per time block

6. The utility program notifier gets the accepted bids from the Utility Information System and sets the accepted and rejected bids in the DRAS. The information concerning the accepted bid includes the following:

- Participant list (account number)

- Accept or Reject

- Load reduction bids per time block (for verification)

7. The DRAS program notifier sends the acceptance or rejection notification to the participant manager via phone, email, and/or page.

\subsection{GBP Maintenance}

This scenario consists of a set of actions which are necessary to maintain the GBP program. Unlike the configuration and execution scenarios, this set of actions is less a prescribed set of steps and more a set of possible actions that may be performed by the various roles at unrelated times.

1a. The utility program operator gets the operation reports. The utility program operator can get the following status information from the DRAS at any time :

- Event status (for all participants)

o current outstanding events

- Load reduction potential based upon all participants in program (optional)

- Feedback from DRAS Clients (for those that have optional feedback)

o event logs

- Bids and signal levels per time block for current events (for all participants, individually and those grouped together)

1b. The participant manager checks status. The participant manager can get the following status information from the DRAS at any time.

- DRAS Client communications status

o current status

o last contact

o current signals levels

o current participant manual control levels (opt-out)

o communications logs

o signal logs

o manual control logs

- Event status (same as above)

- Bids and signal levels per time block for current events 
1c. The utility program operator adds, modifies, and deletes facilities participating in the program. This is similar to the original configuration step.

$1 \mathrm{~d}$. The participant manager opts out of the DR program. At any time, the participant manager can opt out of the DR program on the DRAS. When in an opt-out condition, DR events are not propagated to the DRAS Client. The opt-out can be either for the entire program or a single event. In addition the DRAS does not propagate bids to the utility IT system for participants that have opted out of a DR event.

1e. The participant manager configures a standing bid in the DRAS.

\subsection{Overall Requirements}

This section presents a general list of requirements for the DRAS that may be independent of specific use cases. The general high level requirements include:

- Should use industry accepted methods and standards to ease integration and access to DRAS services from third parties.

- Must follow a well established set of security policies to insure that all exchanges of information are authenticated, private, and maintain integrity of the information being exchanged.

- Must allow easy integration with end user facility IT infrastructure:

o Ease in dealing with firewalls

o Good IT network citizen (i.e. no security risk, insignificant network load, etc).

- The latency of DR events sent from the utility to the end user should be no more than 1 minute, depending upon the configuration of the interaction between the DRAS and DRAS Client.

- The DRAS must maintain accurate time within 15 seconds.

- The DRAS should have a means to allow participants to participate in multiple DR programs and dynamic pricing through the same DRAS.

- The DRAS should recover gracefully from facility faults with minimum lost data. Examples of such faults might be power failures or connectivity loss. 


\section{Specifications}

This section gives specifications for the various components and functions of the DRAS.

\subsection{Automated Demand Response Architecture}

Figures 4 and 5 show the component and system architecture that interface with the DRAS to manage the actual automated DR events. Likewise the Figures show the architecture of the components and systems that interface to the DRAS to automate the submissions of bids by participants in a DR program that requires bidding.

The look and feel and implementation of the User Interface (UI) used to perform various functions on the DRAS communications is outside the scope of this specification. Thus, the DRAS UI Web Server and the Web Client are shown outside the realm of the DRAS. What is standardized is the exchange of information with the DRAS that allows a UI to be built that can be used to view and manipulate the information exchanged with the DRAS. In theory it is possible to support the UI on the DRAS and particular implementations of the DRAS may do this, but how the user interface is implemented is not part of this specification. By using the standardized information exchange specified in this specification, it will be possible for third parties to implement a UI with their own look and feel and still interact with the DRAS in a standards-based fashion.

The figures include a so called "Third Party Notification System." This sub-system is responsible for notifications to facility operators using various existing technologies such as phone, pages, email, fax, etc. The purpose of showing this as a separate component is to highlight the fact that certain types of notifications (such as voice mail) will not be part of the specification and may be provided by third party systems. The systems may be part of the utility infrastructure, but in the most general case they are a standalone service as depicted in the diagrams. At a minimum, the DRAS must support direct e-mail notification to the facility that includes exception handling and bidding information. This is separate from the notifications that may be provided by a third party notification system.

It is important to note in the architecture shown in Figures 4 and 5 that the DRAS itself is depicted as a standalone service from both the utility and participant's IT infrastructure. This is the most general case and is what is used as the main use case in this OpenADR specification. In fact, specific incarnations of the DRAS may be integrated within either the utility or participant's IT infrastructure and services and thus the interfaces to the DRAS may not be implemented as depicted in these figures. 


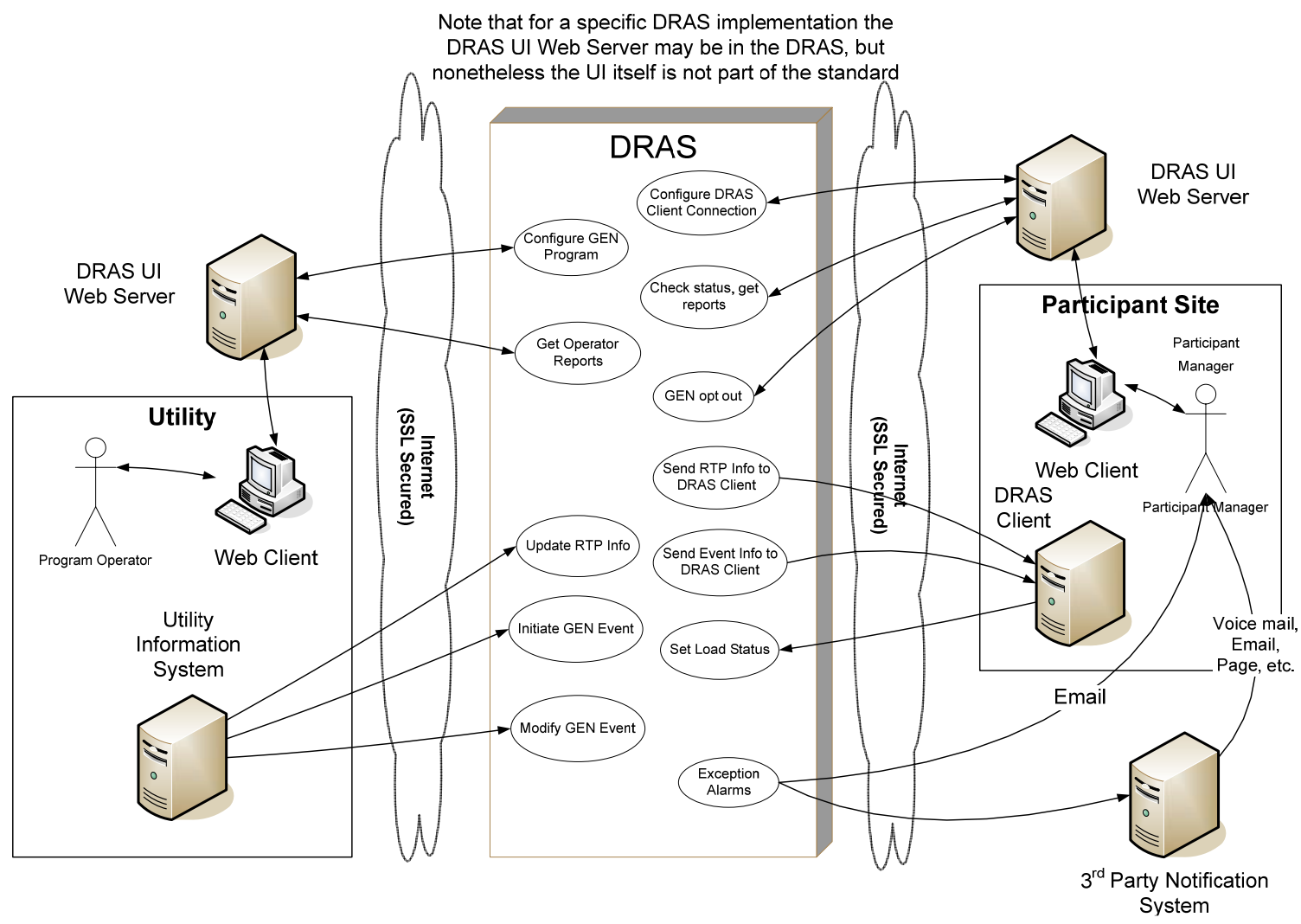

Figure 4. General Automated Events Architecture with Standalone DRAS

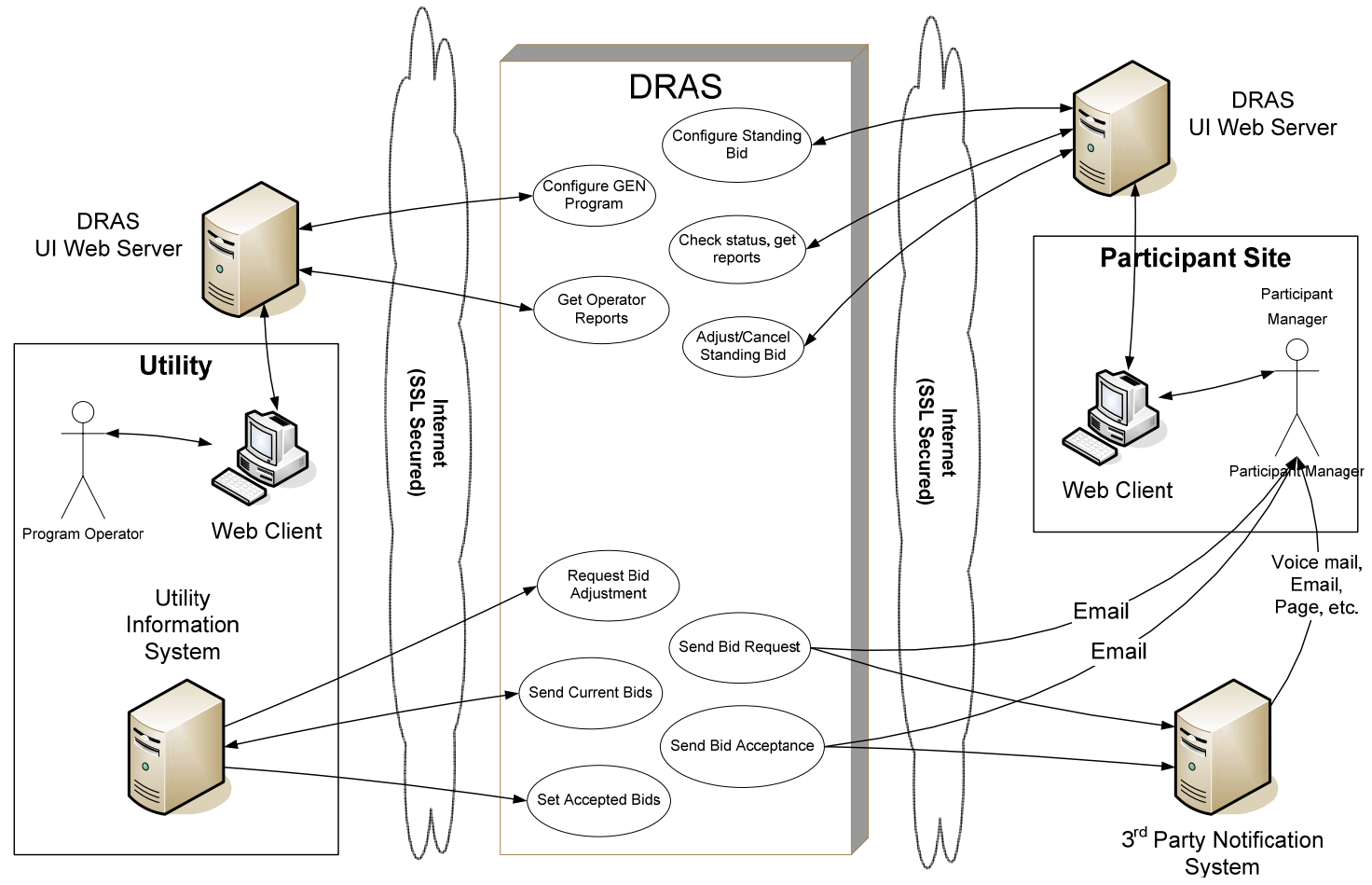

Figure 5. General Automated Bidding Architecture with Standalone DRAS 


\subsection{General Requirements}

In analyzing various use cases and the architectures in the previous section, it is possible to group the various functional requirements as shown in Figure 6.

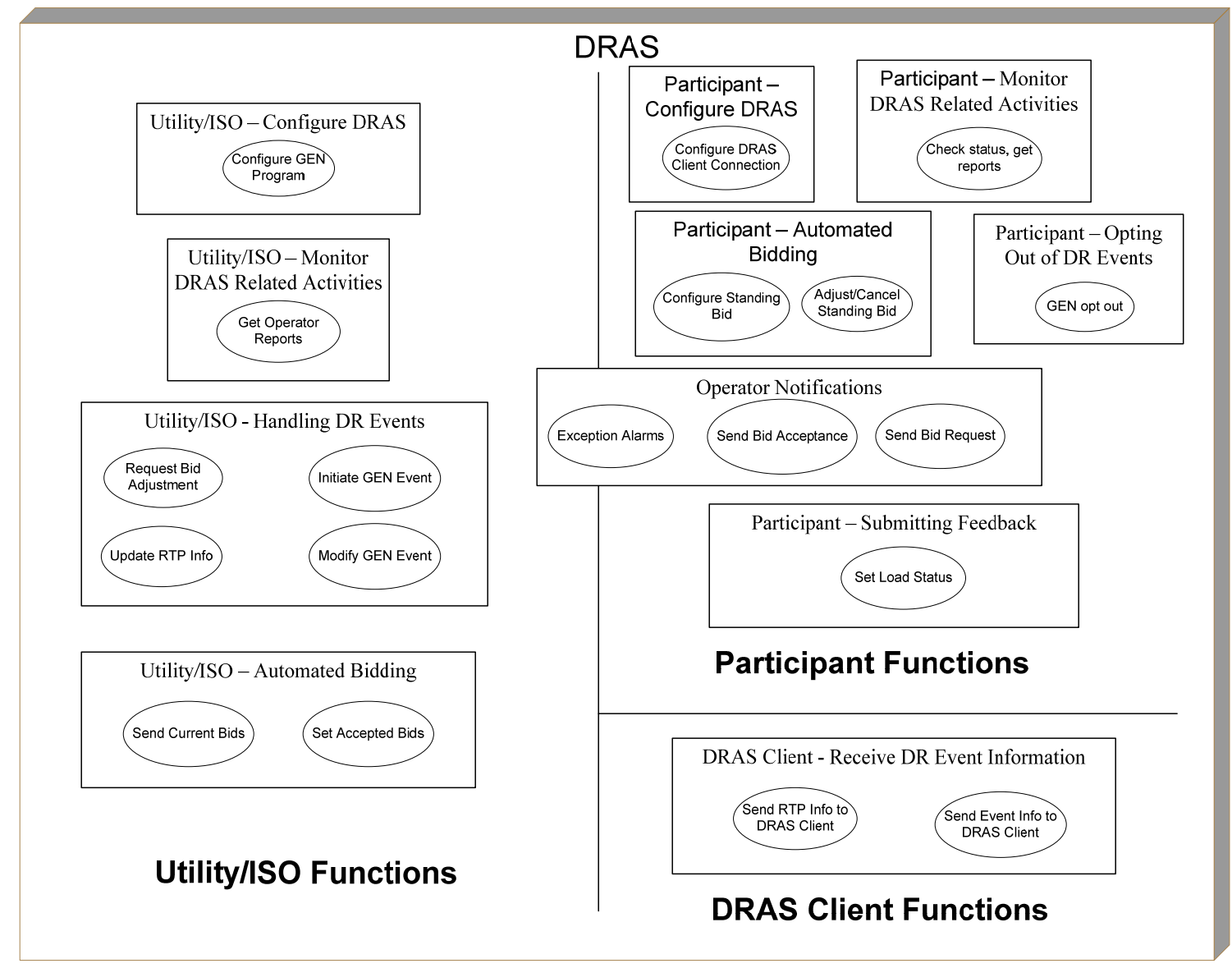

Figure 6. Use Case References to Functional Specification

The interface functions supported by the DRAS can be classified into three groups:

- Utility and ISO functions

- Participant functions

- DRAS Client functions

This classification reflects the role of the DRAS as an integration point between the utility or ISO and the participants and the DRAS Clients. Because the DRAS may not be a standalone entity, but instead may be integrated with a utility's IT infrastructure, it is not a requirement that the DRAS support those functions tagged as utility or ISO functions. In the case where the DRAS is not integrated with the utility IT infrastructure and supplied as a standalone entity, then it should support the interfaces tagged as utility or ISO. 
Furthermore, a particular implementation of the DRAS may contain a Web server that is responsible for serving up a Web-based user interface (UI) to the participant operators. In this scenario it is not a requirement that the participant functions be made available for third parties to implement their own Web-based UI and therefore the methods specified as part of the participant interface need not exist. Instead the functions normally provided by the participant interface will be provided by some sort of UI or tool that is integrated with the DRAS and therefore need not utilize the methods specified in the participant interface. If it is a requirement that the DRAS allows third

parties to build Web-based clients for participants then the methods described as part of the participant interface must exist as specified below.

In any case, the DRAS must support the methods tagged as belonging to the DRAS Client interface.

The participant, utility or ISO, and DRAS Client interfaces are further decomposed into specific methods and covered in more detail below.

In addition to the functional descriptions given below, the data model entities used as parameters for each of these methods are also described. The conceptual data models developed are from the point of view of the utilities, participants, and DRAS Clients and are depicted in the conceptual entity relationship diagram (ERD) in Section 6.4.1.

For the remainder of this document the term function and method are interchangeable and take on their normal meaning, i.e. referring to a software interface construct that is used to execute a certain well defined set of functionality.

\subsection{Common Requirements}

All DRAS functions must satisfy the following requirements:

- They must all be implemented using some type of reliable communications, meaning that it must be possible to determine if information exchanged as part of executing the function was received correctly.

- All functions must adhere to the minimum security policies specified in Section 10 .

- All functions must be implemented using industry standard Web services.

- All functions must restrict access based upon a well documented set of security roles.

\subsubsection{DRAS User Accounts and Security Roles}

For security reasons, each of the functions accessed through the interfaces of the DRAS defined in this specification can only be accessed by users with an appropriate authorization on the DRAS. Further details are described in DRAS security policies. In addition accounts that are created to allow access to functions on the DRAS have one or more security roles. The following types of security roles are used: 
- DRAS Operator-DRAS operators are highly trusted individuals with wide ranging access to all information and functions of the DRAS documented in this specification including creating other users with their appropriate security roles. DRAS operators cannot be created via any of the interfaces documented in this specification.

- Participant Manager-Participant managers have access to all the information associated with a particular participant account. They are created by using the functions described in Section 0.3. Within the context of this document a participant manager may also be referred to as a "Participant Operator".

- DRAS Client-The DRAS Client represents the software agent for the machine to machine communications between the DRAS and the participant facility. DRAS Clients are created as part of the configuration process described in Section 0.2.

- Utility and ISO Operator-Utility and ISO operators have access to all the utility methods and functions in the DRAS. This process is beyond the scope of this document.

- DRAS Client Installer (Technical Coordinators) - These are the individuals that are responsible for installing and testing DRAS Clients in the field. How these accounts are created are beyond the scope of this document.

- Participant manager and DRAS Client accounts are established as part of the configuration of the DRAS as described in Section 0. The DRAS operator, utility program operator, and DRAS Client installer accounts are managed by processes that are outside the scope of this document.

\subsubsection{Logs and Reports}

The DRAS must track and log the items described in this section. In general each of the items logged are accessible by the various operators. In addition the DRAS must monitor exception conditions that result in so called alarms. In general, alarms result in the notification of various operators as described in Section 6.3.3.

DRAS operators and utility program operators may access the logs and alarms of all the transactions regardless of the participant while participant managers may only access the logs and alarms associated with their transactions. Also no one should be permitted to delete logs.

As a practical matter the DRAS cannot keep logs forever since their size will eventually fill up available storage resources. It is therefore not a requirement that the DRAS to keep logs indefinitely. The process to specify the historical DRAS communications logs is beyond the scope of this document. The various items that are logged by the DRAS are detailed below.

\subsubsection{The DRAS Client Communications State}

The DRAS is required to track the communications state of each DRAS Client. A DRAS Client may be in one of the following communications states: 
- Online-the DRAS Client is communicating properly.

- Degraded (online with errors - note that the threshold for this is implementationspecific)

- Communications Failed.

- Out of Service (provisioned offline for testing, maintenance, etc.)

As described by the functions in Section 0, the DRAS Client's communication state can be queried at any time.

\subsubsection{All Transactions with the DRAS}

The DRAS must log all invocations of functions as part of its normal operation. The information recorded is specified by the TransactionLog entity as described in the data model section. As described in Section 0 the DRAS Client's transaction logs may be queried at any time.

\subsubsection{Exceptions and Alarm Conditions}

The following alarms must be tracked by the DRAS.

- DRAS Client communication going ON or OFF line.

In general alarms result in operator notifications as described in Section 6.3.3. In addition alarms must be logged by the DRAS so that they may be queried using the functions described in Section 0.

\subsubsection{Operator Notifications}

The DRAS may send notifications to various human operators under a variety of circumstances. In order to notify the human operators the DRAS must support email notification and may support interfacing to third party notification systems. Third party notification systems include pagers, voice mail, text messages, etc. The process of how the DRAS interfaces to third party notification systems is beyond the scope of this document.

In general, participant operators and utility program operators may be sent notifications by the DRAS. The email contact information for a participant operator is specified in the ParticipantAccount object for that participant as described in the data model section. The utility program operator's email contact information is specified when their account is set up by the DRAS operator. The process to specify the format of the email messages that are sent to the various operators is beyond the scope of this document.

The DRAS must send the following notifications:

- DRAS Client ON or OFF line. The DRAS must track when a DRAS Client goes ON or OFF line and when it does it must send an email notification to the following operators: 
o The participant operator(s) who manages that DRAS Client if so configured to receive notifications.

o The utility program operator who configured the participant account to receive these notifications.

o All DRAS operators.

- DR event initiated. When the utility or ISO issues a DR event or modifies an existing DR event then a notification must be sent to all participant operators that are associated with DRAS Clients that would normally receive that event. Note that the program may require bidding by the participant in which case this notification may be a request for a bid.

- Participant bids accepted or rejected. When a participant's bids have been accepted or rejected the participant operator must be notified.

\subsubsection{Testing}

The DRAS must support the testing of DR event configurations and communications with DRAS Clients. It must support the following types of tests:

- End to end testing of DR events. It supports this by allowing the utility or ISO to initiate so called test events. These are treated like any other DR events by the DRAS including sending the DR event information to DRAS Clients. The only difference is that there is a field in the DR event information that signifies that the event is a test event. When the DRAS Client receives test events it may choose to deal with it in whatever fashion is appropriate for the test scenario in the facility, including ignoring it.

- DRAS Client testing. There is a way for DRAS Client installers to take a DRAS Client off line in the DRAS and send it test messages. The DRAS Client installer has the ability to set various state variables that are used by DRAS Clients so that they can be manually controlled. See Section 7.3.6.

\subsection{Introduction to Data Entities Used By Interface Functions}

This section gives a brief description of the various data entities used for information exchange via the various functions described in this specification. This section is only meant to give a brief introduction and conceptual overview of the various data entities.

The conceptual data model represents the various data elements that are part of the DRAS operation as they are viewed from the point of view of the utility or ISO and the participants. It is not intended to represent a specific database schema that is implemented in the DRAS but rather represent the various data elements that are used by, the utility or ISO, participants, and DRAS Clients when they interface to the DRAS.

The conceptual data model is represented by several Entity-Relationship (ER) diagram. Each diagram is a collection of entities and the relationships between them. Each Entity represents a basic data element with the following characteristics: 
- Name of the data entity

- Primary Key of the entity ("PK"). This is an identifier that represents how the entity may be referenced by other entities.

- Data elements or attributes. These are the various fields of the entity.

- References to other entities. Often a particular entity will refer to another entity, represented by the foreign key ("FK"). These foreign keys are the primary keys of the entities that they refer to.

\section{Key to Interface Function Figures}

Each discussion in this section focuses on those entities that are shaded in the corresponding reference figures. Un-shaded entities have been defined elsewhere as past of another process. Furthermore, attributes in bold are required while the others are optional. Arrows are used when one entity refers to another. The text label on the arrow notes the type of relationship between the two entities. The number sequence associated with the arrow specifies how many of the entities are referred to in the relationship, for example:

- $1 . .^{*}$ means at least one but possibly many (one to many)

- 1 means exactly one (one to one)

- * means zero to many

Note that while this section is relatively descriptive in nature, the name given for each of the entities and their accompanying fields and attributes match the names are given in Section 8, which contains a more detailed description of the schemas for each of the entities presented here.

\subsubsection{Data Entities in Support of Utility and ISO Use Case Actions}

The entities in this section are specifically used by the various operations in support of the utility or ISO functions. As such they are organized according to the various functions that may be performed.

\subsubsection{Utility Issues DR Event}

These are the entities used when the Utility or ISO initiates a DR event. In particular the UtilityDREvent entity is used to specify all the information associated with a DR event and contains the following general attributes:

- eventIdentifier-This is a globally unique id that is specified by the utility or ISO when the DR event is issued. It is subsequently used to associate and retrieve information related to a particular DR event.

- programName-This is an identifier that specifies which DR program the DR event is being issued for.

- eventModNumber-This is a sub identifier to the event identifier and is used specifically to determine when changes have been made to the DR event information since the last time it was issued. For example, the very first time a 
DR event is initiated by the utility or ISO, this has a number of 0 and will continue to have a value of 0 for each and every subsequent transmission unless the DR event is subsequently modified by the utility or ISO. At that time this number is increased to the next version number, indicating the original event was modified.

- utilityITName-This is an optional field which is the name and/or version number of the utility IT system that initiated the DR event.

- destinations-This is a list of identifiers that specifies who is to receive the DR event. Note that it can be any of the following:

- Explicit Participant Account User ID(s) (uid)

- Group identifiers

- DRAS Client location specifications

- eventTiming-Various timing parameters for the event, including:

- notificationTime-This is the time at which the participants should be notified of the DR event.

- startTime-This is the date and time that the DR event becomes active.

- endTime-This is the date and time that the DR event ends.

- biddingInformation - If a program supports bidding by the participants then the following fields are also included.

- openingTime-This is the time at which the participants may start placing bids.

- closingTime-This is the time at which the bidding will close and is the deadline for which the utility may receive the bids from the DRAS.

- Event Information-This is the information associated with the DR event and is a list of EventInfoInstance entity as described below. 


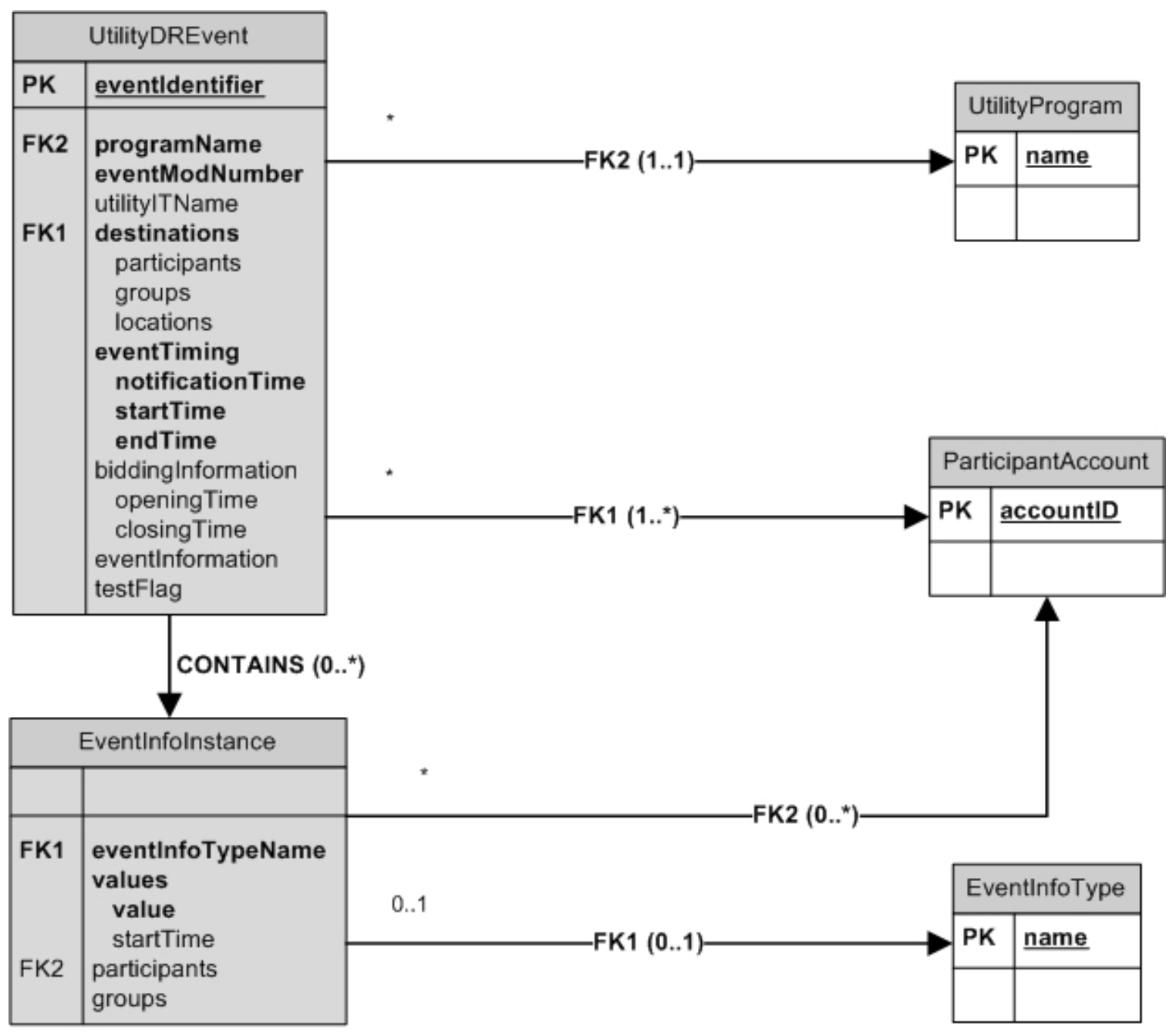

Figure 7. Utility Issued DR Event Entity

Each UtilityDREvent may contain a list of EventInfoInstance variables which are used to describe the information that may accompany a DR event. Each EventInfoInstance entity contains the following general attributes:

- eventInfoTypeName-this is a name that is used to correlate this value against a predefined type of values for this program as described in Section 6.5.3.3.2. Note that the type name provides a way for the various values associated with a DR event to be identified. The various types are defined when the DR program is defined and given names which are referred to here.

- values-these are a list of one or more values of the defined type. If there is more than one value then each value corresponds to a specific time slot within the DR event ACTIVE period. Essentially they will form a schedule of values. This is described in more detail in Section 6.5.

- participants-this is a list of participants for which these values apply. It allows the information associated with a DR event to be applied to specific participants. 
- groups-this is a list of groups for which these values apply. Since participants may belong to a group, groups are another way of specifying which participants are to receive the information.

As can be seen by the diagram it is assumed that the following entities exist before an UtilityDREvent is created that refer to them:

- UtilityProgram

- ParticipantAccount

- EventInfoType

\subsubsection{Utility Configuration of DRAS}

When the utility or ISO configures the DRAS, it uses the entities shown in Figure 8.

The UtilityProgram entity represents all the information associated with a DR program that is created by a utility or ISO. Each program has a set of attributes that describe how the program is managed and run from the point of view of the DRAS and participants. The attributes include the following type of information:

- A name.

- A ProgramConstraint entity in the form of time and date attributes which specify when a DR event can be issued.

- List of participants that participate in the program.

- Parameters that control how bidding is performed by a participant as part of the program.

- Specifications for the type of information that can be associated with a DR event in the form of EventInfoType entities.

- A priority for the program in relation to other programs.

The EventInfoType entities are part of the UtilityProgram entities and are used to specify the type of information that may be associated with a DR event when one is issued. Examples of this kind of information may include things like real time prices, shed or shift levels, etc. An EventInfoType contains the following general attributes.

- A name.

- A specification for the data type of the information.

- A specification for constraints on the allowable values such as minimum and maximum values.

- A specification for a schedule if the values may change according to some fixed schedule during the course of a DR event. An example might be prices where there is one price for the first hour of the DR event, a different price for the second hour, and yet another price for the remaining time in the DR event.

EventInfoType entities are described in more detail in Section 6.5. 
The ParticipantAccount entity contains all the attributes associated with a participant. It contains the following types of information:

- Participant name.

- Access credentials (e.g. user name and password) for accessing the functions of the DRAS. Note that a ParticipantAccount may have multiple DRAS Clients associated with their account and each of the DRAS Clients will have access credentials are that are distinct and separate from the access credentials specified here.

- Groups that the participant may belong to. Groups are a way to refer to more than one participant for various operations of the DRAS.

- Programs and dynamic pricing activities that the participants may participate in.

- ProgramConstraint variables associated with how the participant may participate in a Program.

- DRASClient entities that describe the DRAS Clients that the participant will use to communicate with the DRAS.

- Bidding information for any standing bid that the participant may set up.

- Contact information for the DRAS to send notifications to the participant operators.

The ParticipantAccount entity is created by the utility and various fields may be edited by the participant. See Section 8.5 for a more detailed description of the ParticipantAccount entity. 


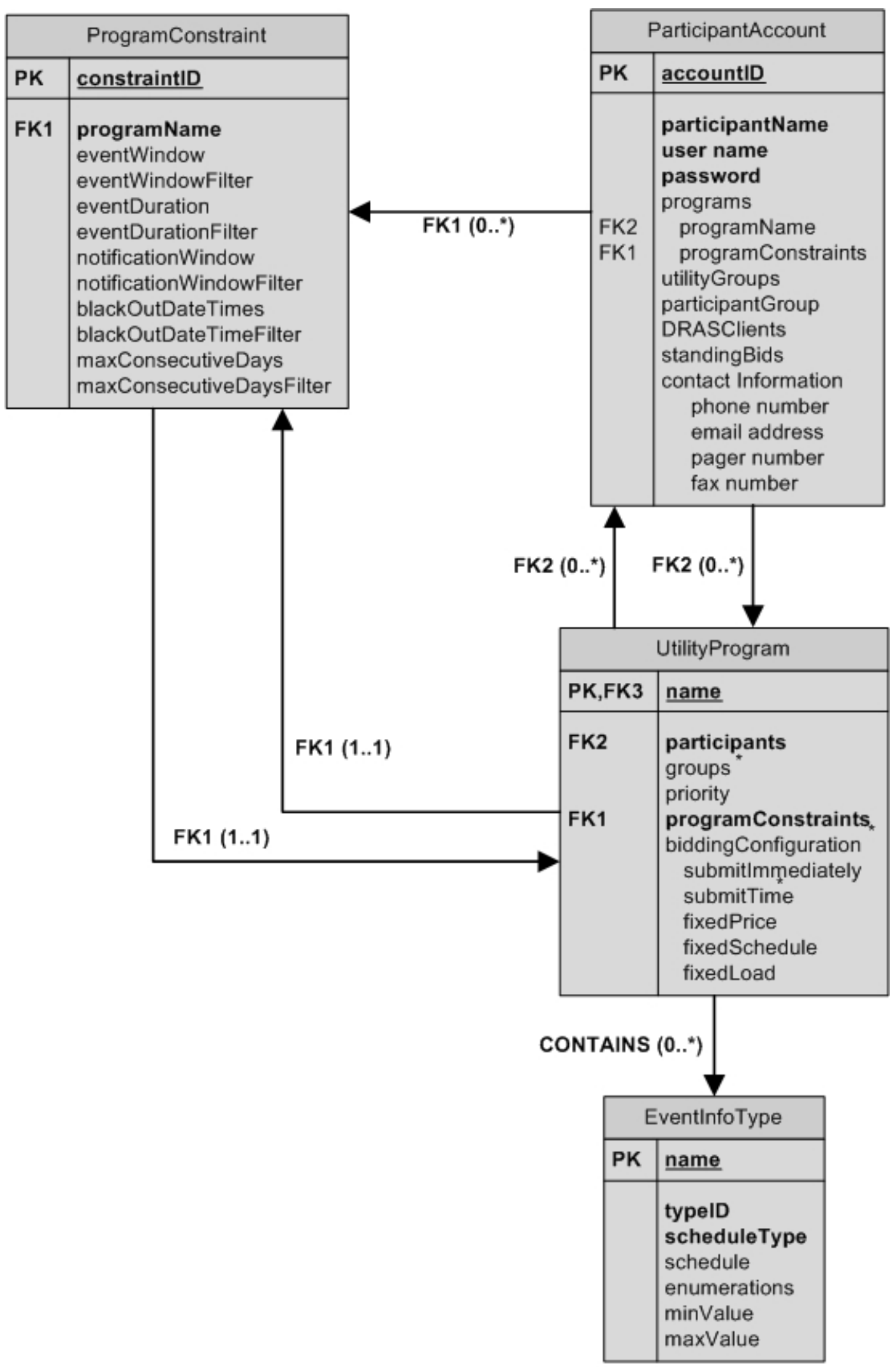

Figure 8. Utility Configuration Entities 


\subsubsection{Utility Manages Bids}

When a participant submits bids to participate in a DR event, those bids are sent to the utility or ISO. The data entities used to represent the bids are described in Section 6.4.2.2.

\subsubsection{Utility gets Logs and Alarms}

The DRAS is required to maintain logs and track alarms that record a variety of activities associated with the DRAS. The data entities used to represent these activities are show in the conceptual ER diagrams in Figure 9. Each entity contains enough information so that it is possible to determine who, when, and what was involved. These are described in more detail in Section 8.7.
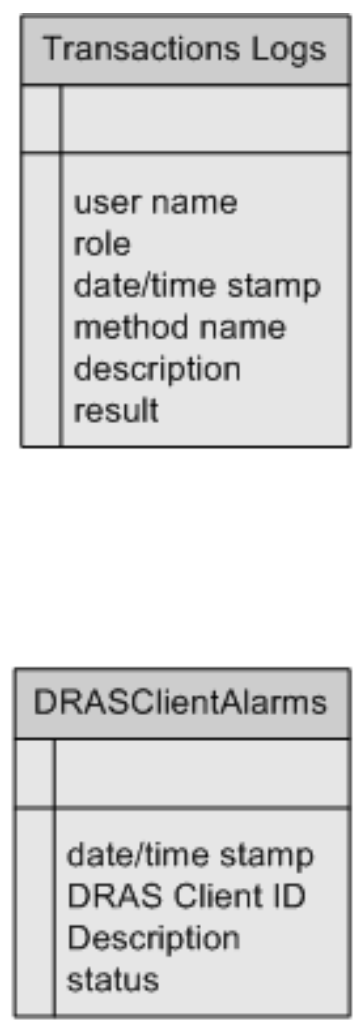

Figure 9. Utility Logs and Client Alarms

\subsubsection{Data Entities in Support of Participant Operator Functions}

\subsubsection{Participant Configuration}

The conceptual ER diagram, Figure 10, shows the various entities that may be created and configured by the participant. They represent the pieces of data that are used for various operations involving the participant.

The utility or ISO creates a ParticipantAccount. The participant may edit only a subset of the attributes associated with this entity. See Section 8.5 for a detailed description. 
The participant may define a number of ProgramConstraints. Each of these is associated with a particular program and defines limitations of how a participant may participate in that program. Note that the ProgramConstraints which are defined for a particular participant are intended to customize the various schedule attributes of how a participant participates in a program and are distinct from the ProgramConstraints that a utility may define globally for a program. The ProgramConstraints for the participant supersede the ProgramConstraints that are defined for the program as a whole. See Sections 6.5.2.2 and 8.4 for a detailed description of this entity.

A ParticipantAccount may have a number of Bid entities that represent standing bids associated with specific programs that may require bidding. See Sections 6.6 and 8.11 for a more detailed description of this entity.

A ParticipantAccount may also define a number of DRASClient entities. A DRASClient entity represents the information associated with a DRAS Client and includes the following types of information:

- Type of DRAS Client-Simple or Smart type.

- Programs that the DRAS Client may participate in.

- ProgramConstraints that define how the DRAS Client will participate in programs.

- Communications parameters that define how the DRAS Client communicates with the DRAS.

- Location information.

- ResponseSchedule entities for the various programs and dynamic pricing that the DRAS Client may participate in if the DRAS Client is a Simple type.

See Section 8.10 for a more detailed description of a DRASClient entity. See Section 6.5.3.2 for a more detailed description of Simple and Smart DRAS Clients.

The ResponseSchedule entities are used by Simple DRAS Clients and represent the translation of the DR event information into simple levels and status. As shown in the Figure 10 a ResponseSchedule entity is a collection of OperationStates. See Sections 6.5 and 8.3 for a more detailed description. 


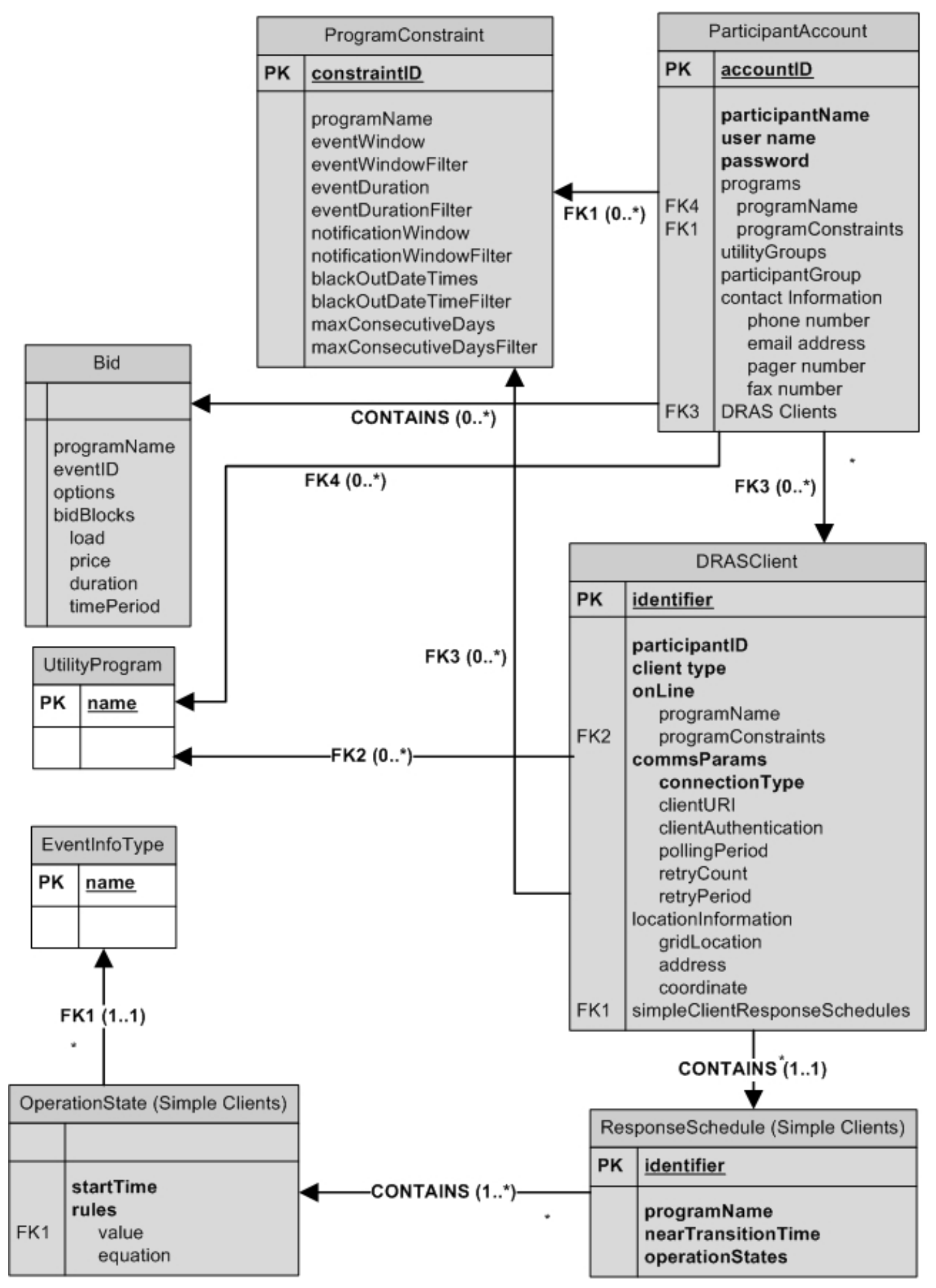

Figure 10. Participant Configuration Entities 


\subsubsection{Participant Operator Submit Bids}

When a DR event is issued by the utility or ISO requiring bidding then the participant uses the Bid entity to submit those bids. The Bid entity is described in more detail in Section 8.11.

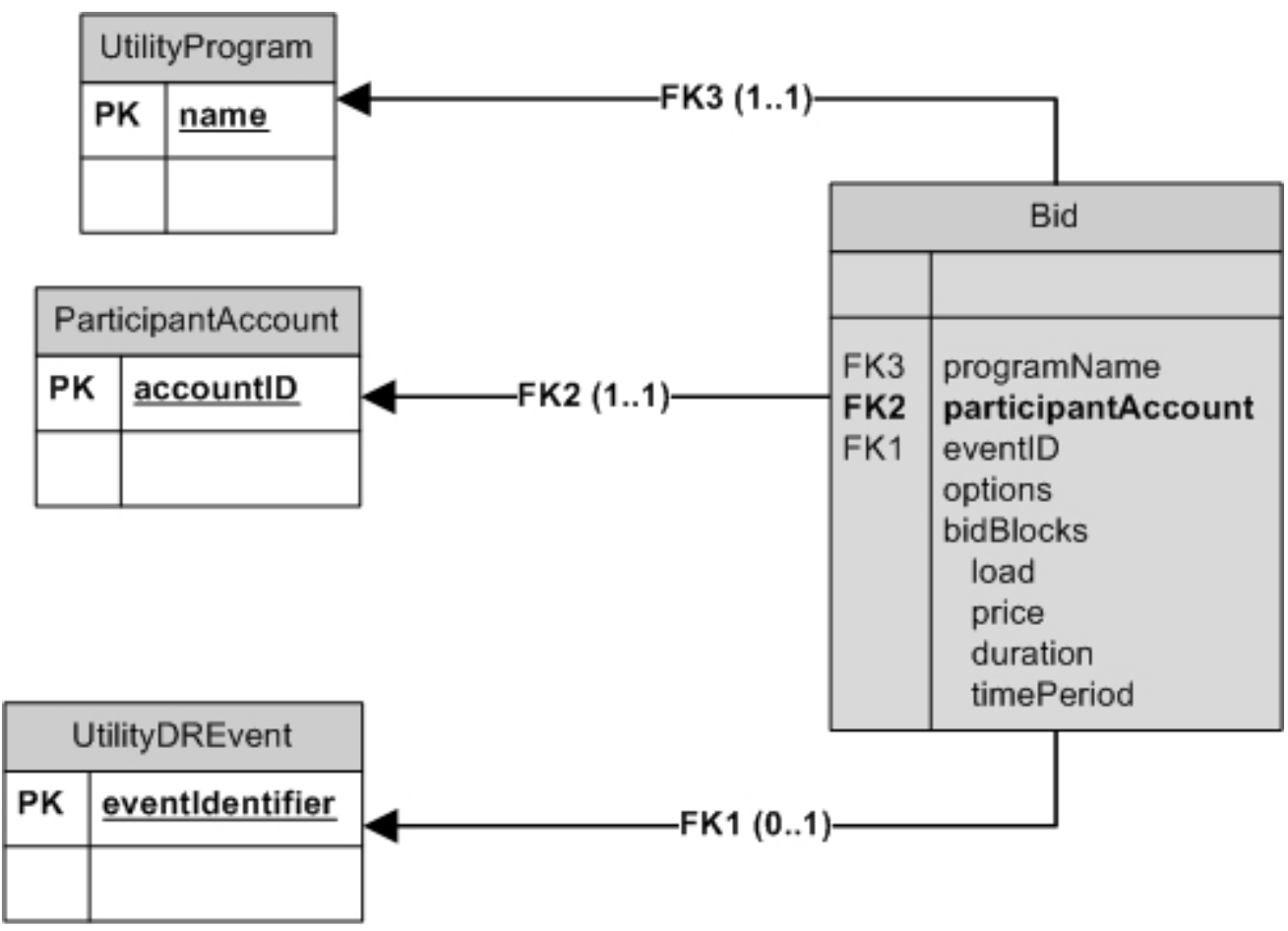

Figure 11. Participant Submit Bid Entity

\subsubsection{Participant Operator Opt-out State of DR Events}

A participant may choose to opt-out or override participating in DR events. The participant does this by using the OptOutState entity to set up one or more conditions within the DRAS that define when the participant will not participate in a DR event. The OptOutState may have the following attributes:

- Which DR programs the conditions apply to. If not specified, it defaults to all programs.

- Which DRAS Clients the conditions apply to. If not specified, it defaults to all DRAS Clients.

- Which DR event the conditions apply to. If not specified, it defaults to all DR events.

- A schedule which defines when the conditions apply.

Note that there can be more than one such set of conditions established by the participant. See Section 8.6. 


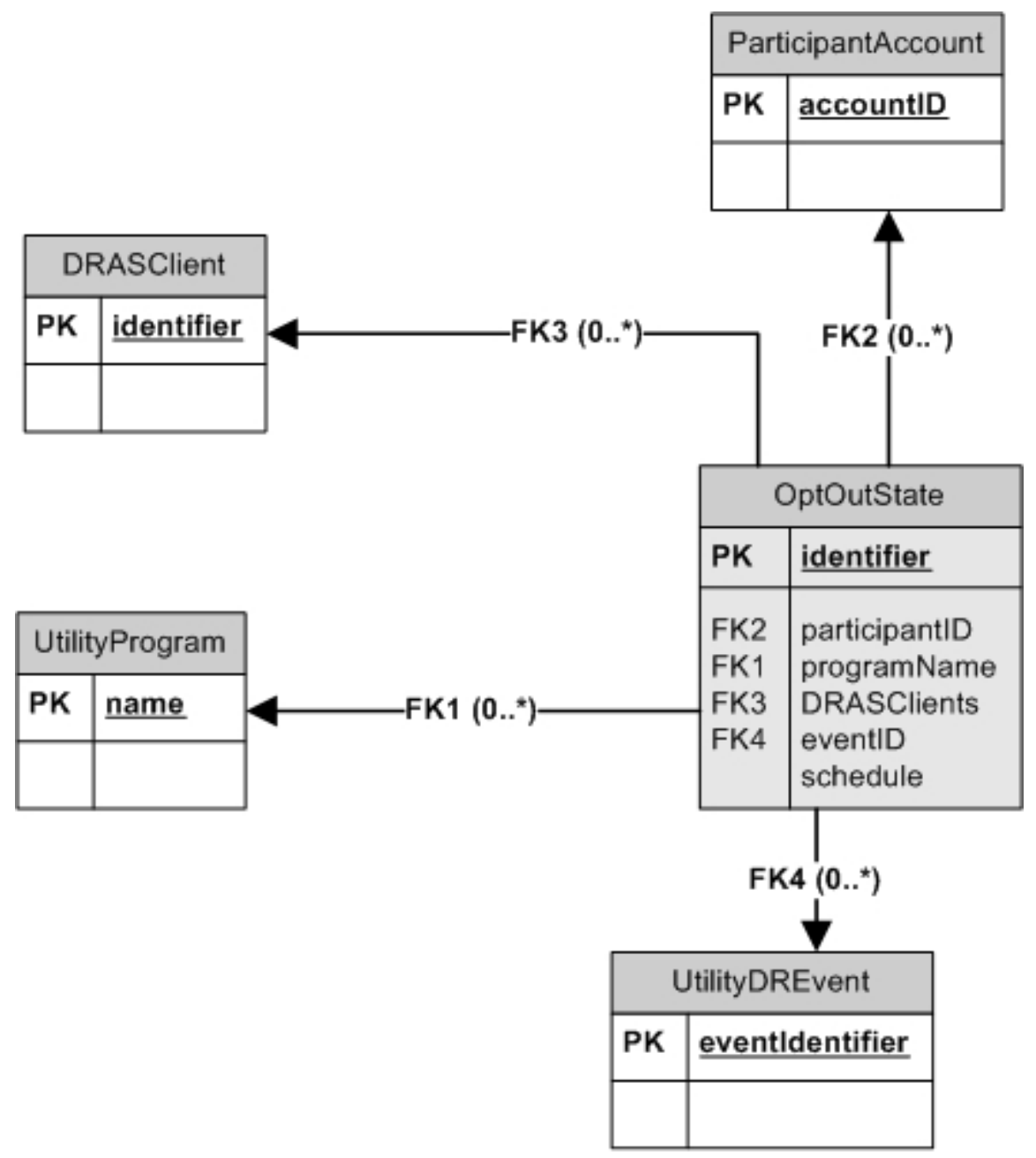

Figure 12. Participant Opt-Out Entities

\subsubsection{Participant Operator Submits Feedback}

The participant may use the ParticipantFeedback entity to notify the DRAS about various conditions or information related to the participant. Examples include demand data and how the participant is responding to a DR event. The Feedback information can be read by the utility or ISO. See Section 8.8 for a more detailed description of the ParticipantFeedback entity. Note that ParticipantFeedback may be submitted by various roles within the system both human and machine. This entity would be used by all such transactions to submit the feedback. 


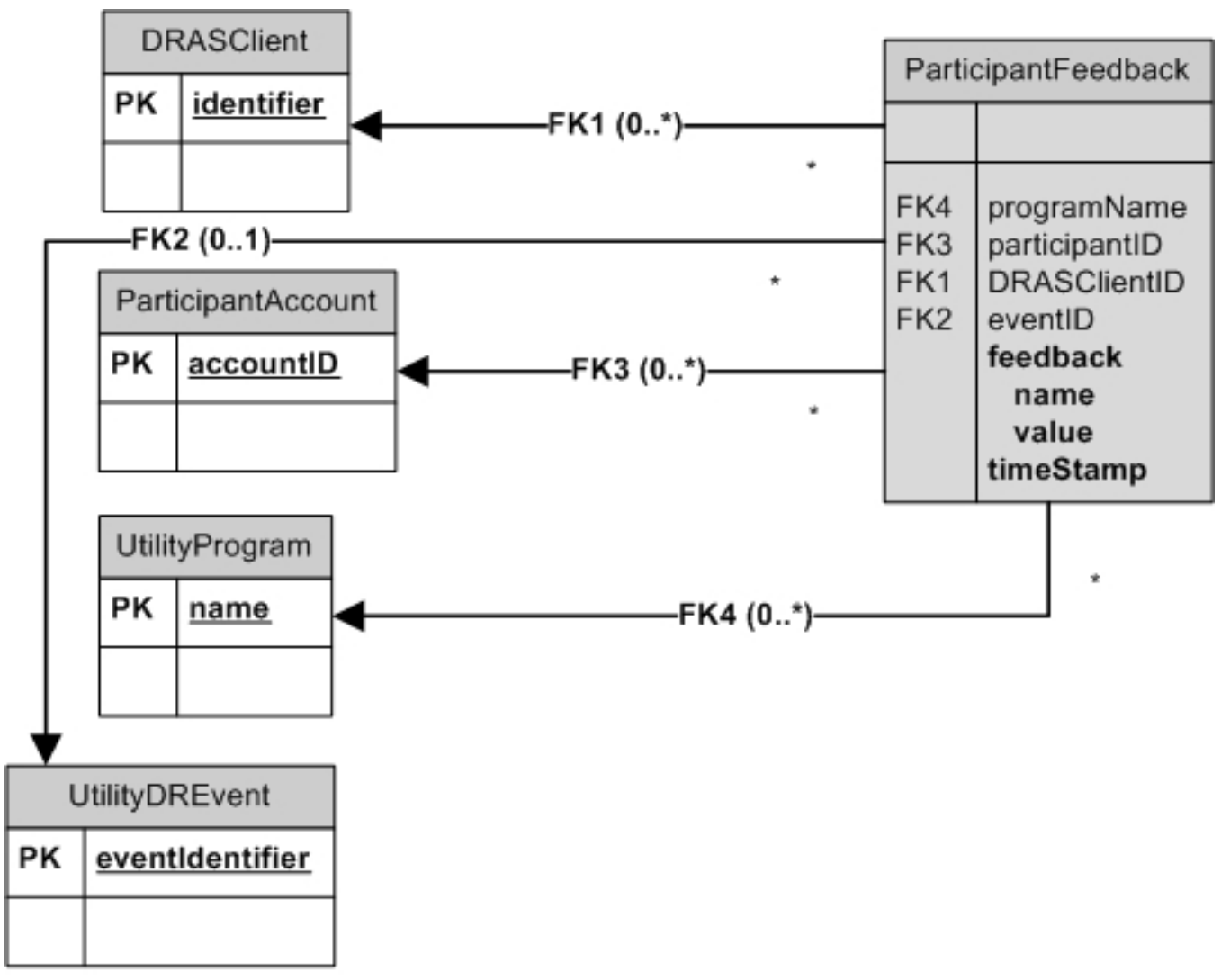

Figure 13. Participant Feedback Entities

\subsubsection{Participant Operator gets Logs and Alarms}

The DRAS is required to maintain logs and track alarms that record a variety of activities associated with the DRAS. The data entities used to represent these activities are show in the conceptual ER diagrams in Figure 14 (repeated here for reference; Figure 14 is the same as Figure 9). Each entity contains enough information so that it is possible to determine who, when, and what was involved in a DR event. These are described in more detail in Section 8.7. The participant operator is only allowed to access logs for which they have access rights, i.e. those items related to their ParticpantAccounts. 

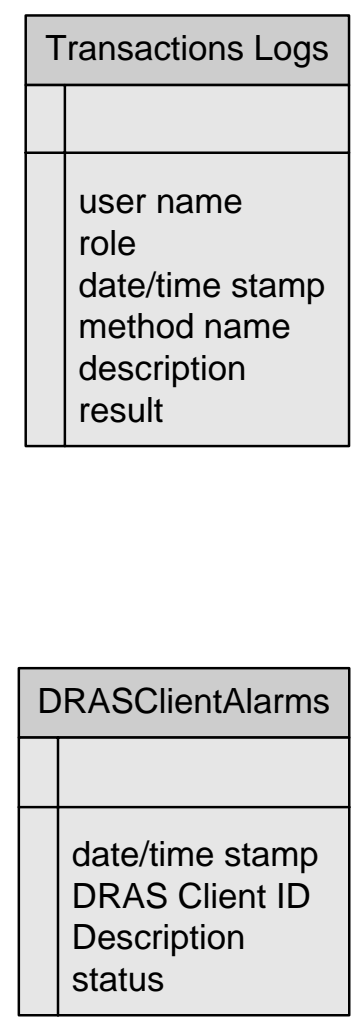

Figure 14. Client Alarms and Utility Logs

\subsubsection{Data Entities In Support of DRAS Client Functions}

As shown in the conceptual ER diagram, Figure 15, the interaction between the DRAS and the DRAS Client involves two entities. The first is the EventState which is a representation of the "state" that the DRAS Client may be in with respect to a DR event. This entity is sent from the DRAS to the DRAS Client. The second is a confirmation message (EventStateConfirmation) or acknowledgement that the DRAS Client sends to the DRAS to notify the DRAS that it has received an EventState message. Note that most of the fields in the EventStateConfirmation are simply copies of the fields from the EventState message that it is replying to. Note that the DRAS Name field provides a means for DRAS Clients to interact with more than one DRAS even though each DRAS may have no knowledge or interdependencies between them.

The EventState contains all the relevant information that describes a specific DR event to the DRAS Client. The same EventState entity is used for both Simple and Smart DRAS Client types. The EventState is described in more detail in Sections 6.5.3 and 8.12. Note that specific Web service interfaces may have a slightly different schema for the EventState as described in Section 9.3. 


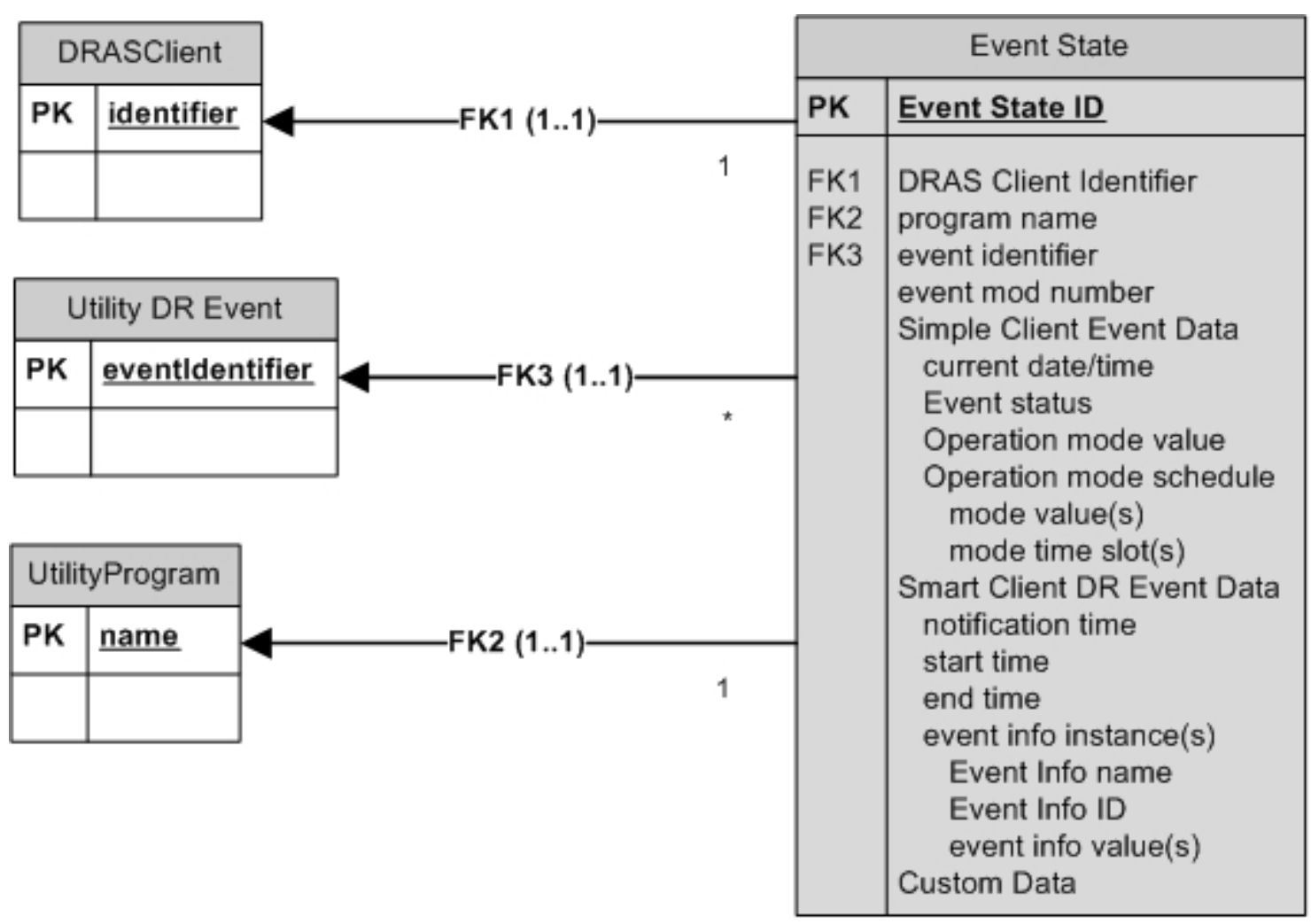

\begin{tabular}{|l|l|}
\hline \multicolumn{2}{|l|}{ Event State Confirmation } \\
\hline PK & Event State ID \\
\hline & $\begin{array}{l}\text { DRAS Client Identifier } \\
\text { program name } \\
\text { event identifier } \\
\text { event mod number } \\
\text { current date/time } \\
\text { operation mode value } \\
\text { opt-in } \\
\text { Event State ConfirmationCol1 } \\
\text { DRAS Name }\end{array}$ \\
\hline
\end{tabular}

Figure 15. DRAS Client DR Event State Entities

\subsection{DR Event Models}

This section details how DR events are viewed and modeled by the utility or ISO that initiate the events and the DRAS Clients that receive the events.

The general assumptions concerning the issuing of DR events within the DRAS include:

- The DRAS is assumed to be managed by a single business entity. While there may be multiple utility or ISO operator accounts, there is only a single business 
entity that is responsible for issuing DR events. This means that if there is a DRAS that is managed by a third party there cannot be more than one utility or ISO that can issue DR events through that DRAS. This assumption is designed to avoid conflicts between events and other issues that should be resolved by the entity that is issuing the DR events.

- Only one event for a specific DRAS Client may be active at any time. See below for a more formal definition of an "active" event. There may be multiple events issued and pending, but only one of them will be active.

- DR programs may have a priority associated with them.

- DR events with overlapping active periods may be issued, but only if they are from different programs and dynamic pricing and only if the programs have a priority associated with them. DR events for programs and dynamic pricing with higher priorities supersede the events of programs and dynamic pricing with lower priorities. If two programs and dynamic pricing with overlapping events have the same priority then the program whose event was activated first takes priority.

\subsubsection{Utility or ISO View of a DR Event}

When the utility or ISO initiates a DR event it uses an UtilityDREvent entity which contains the following general attributes:

- The DR program the event is for.

- Time and date parameters concerning when the event will take place.

- Time and date parameters concerning when participants should be notified of the upcoming event.

- Who and/or where to send the DR event information.

- Information associated with the event (EventInfo). This is program specific information that is related to the event, e.g. RTP or shed or shift level.

\subsubsection{DR Event Time Parameters and States}

Figure 16 shows the different time parameters associated with DR event states. The participants are notified of an upcoming event at the Issue Time (A).The time period between the Event Start Time (B) and the Event End Time (C) is known as the "ACTIVE" state for the DR event. The "ACTIVE" state for the DR event is the only state or time during which the EventInfo is valid. The time period from the Issue Time (A) till the Start Time (B) is the "PENDING" state of the DR event. The "PENDING" state of the DR event is the time during which the DR event is pending, "IDLE" periods, with respect to this DR event, are the times before the Issue Time (A) and after the End Time (C). Event Information (D) is the information associated with a DR event - See Section 6.5.1.2 for what information is included. Another way to look at state transitions is provided in Figure 17; this figure is described in detail in Section 6.5.3.4. 


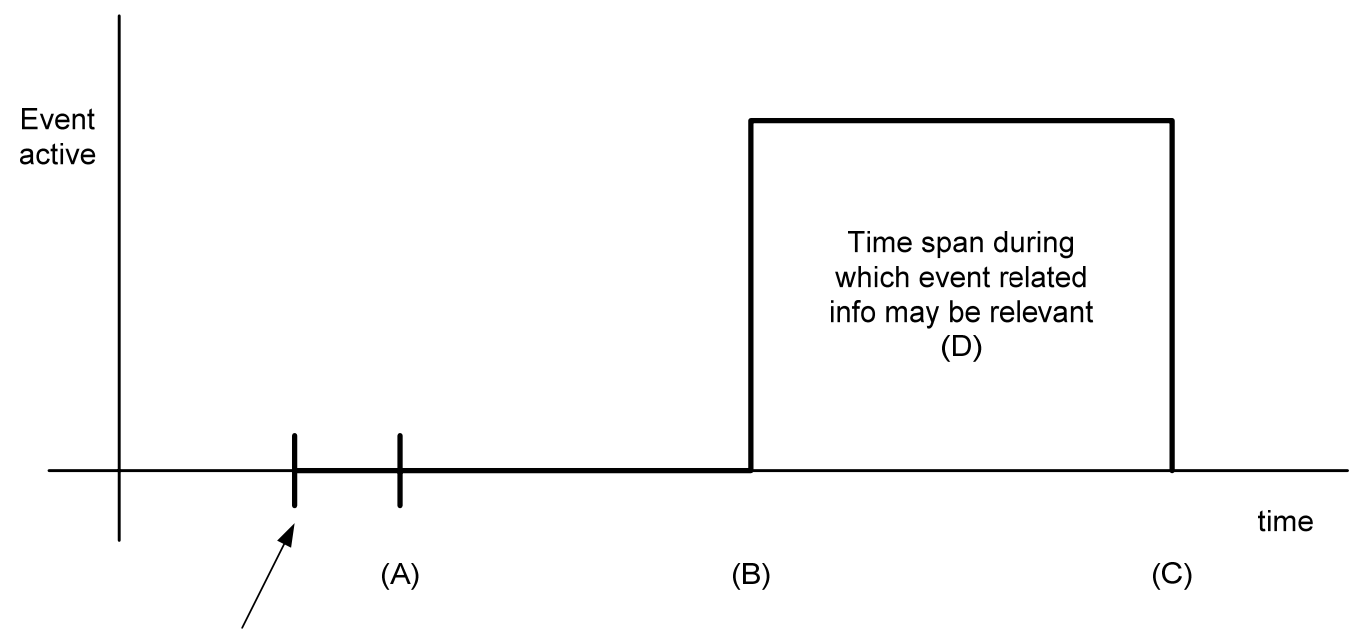

DR Event sent to

DRAS

Figure 16. DR Event Model (Utility or ISO View)

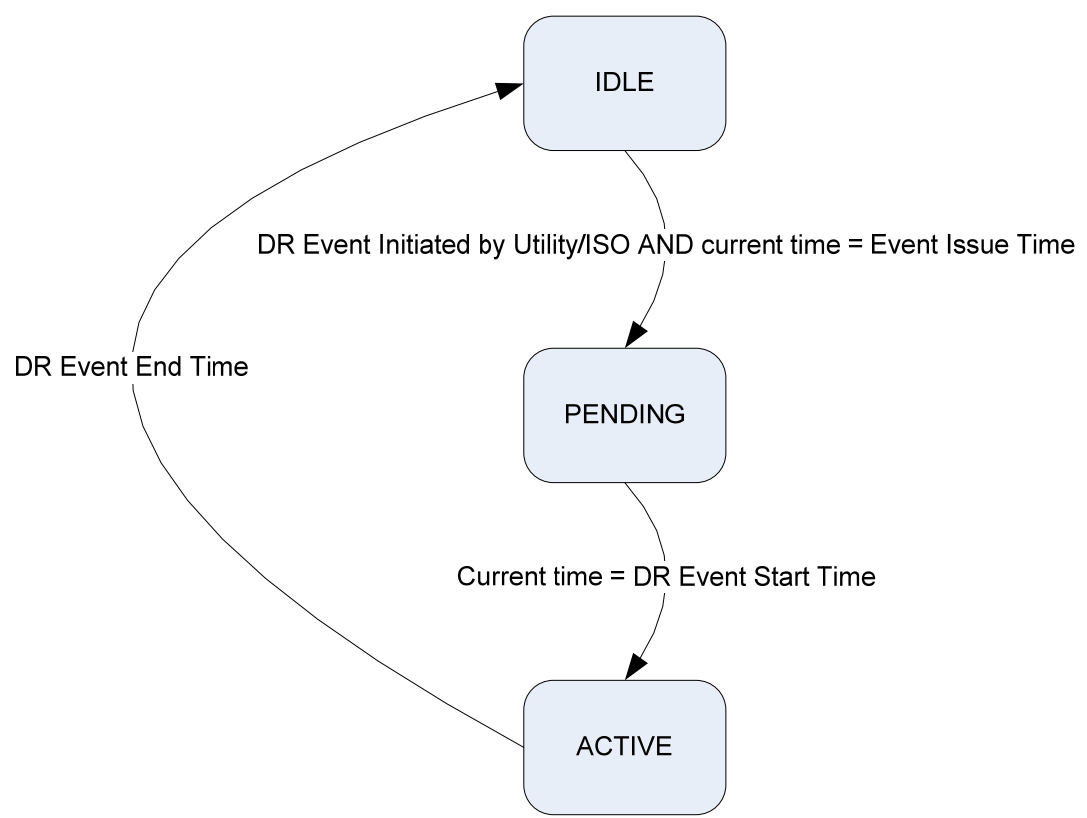

Figure 17. State Transition Diagram

\subsubsection{DR Event Information}

DR programs and dynamic pricing are typically designed to use a variety of information to cause reactions by participants to DR events that are issued by the utility or ISO. In some cases prices are used to trigger responses to the DR events while in other case it might be a shed or shift level. In general there can be a wide range of different types of information associated with a DR event depending upon how the DR program was designed. Therefore the data models used to describe the information associated with 
DR events are designed to accommodate the wide range of information that may be associated with a DR event. This information is represented by EventInfo entities.

When a program is defined within the DRAS there are specifications associated with the program that define what type of information may be associated with a DR event when one is issued for that program. Each type specification for an EventInfo is referred to as an EventInfoType. A program may be defined that allows for multiple different types to be associated with a program. Each EventInfoType contains the following attributes and elements.

\section{EventInfoType}

- name-this is the name of the type of event in use. Analogous to a variable name.

- typeID-this identifies the type of information and may take on one of the following values:

o PRICE_ABSOLUTE - Price number, i.e. $\$ 0.25$

o PRICE_RELATIVE - Change in price, i.e. $-\$ 0.05$

o PRICE_MULTIPLE - Multiple of current price, i.e. 1.5

o LOAD_LEVEL - Amount of load based on an enumeration, i.e. moderate, high, etc.

o LOAD_AMOUNT - Fixed amount of load to shed or shift, i.e. $5 \mathrm{MW}$

o LOAD_PERCENTAGE - Percentage of load to shed or shift, i.e. 10\%

o GRID_RELIABILITY - Number from 0-100 signifying the reliability of the grid. 100 signifies the highest level of reliability while 0 is the lowest.

- scheduleType - This specifies how a schedule may be associated with the DR event information is defined and may take on the following values:

o NONE-there is no schedule and thus EventInfo does not change values during the entire DR event ACTIVE state.

o DYNAMIC-The time schedule is not fixed during configuration, but can be set when the DR event is issued.

o STATIC-The schedule is fixed when the DR program is configured within the DRAS

- $\quad$ schedule-If the scheduleType is STATIC, this is the configured schedule. A schedule is a sequence of time slots that are valid over the entire ACTIVE period of a DR event.

- enumerations - This is a list that defines a fixed set of values that the EventInfo instance may take. If defined, the EventInfo instance is an enumeration and can take on any of the values in the list. If left undefined, the EventInfo instance can take on any contiguous value between the minValue and maxValue.

- minValue-minimum possible value of an EventInfo instance.

- maxValue-maximum possible value of an EventInfo instance. 
Note that when a DR event is issued the EventInfo instances that are associated with the DR event may take on values that change according to some schedule during the ACTIVE state of the DR event. Also note that the schedule that defines when these values change may be defined as part of the definition of an EventInfoType or it may be defined when the DR event is issued. See Section 8.9 for a detailed description of the EventInfoType schema.

How the EventInfoTypes are used by the DRAS Clients varies depending upon whether it is a Simple or Smart DRAS Client. See Section 6.5.3 for a more detailed description of how DRAS Clients view DR events and how they use the EventInfo instances and types of information.

\subsubsection{Propagation of DR Events by the DRAS}

The propagation of DR events from the utility or ISO to the DRAS Clients is controlled by a number of data entities and parameters that are configured by the utility or ISO and/or the participant. In general there exists the following hierarchy:

- Programs and dynamic pricing (DR events are issued as part of a program)

- Participants which belong to programs

o DRAS Clients which belong to participants and programs

When a DR event (UtilityDREvent) is issued there is a so called "destination" attribute which specifies which DRAS Clients will ultimately receive the DR event information. The destination specification may take on one or more of the following attributes:

- Participants-this is a list of participants that reference ParticpantAccount entities that should receive the DR event. If a participant is specified then it is assumed that all the DRAS Clients that belong to that participant will receive the DR event. The exceptions are those DRAS Clients that explicitly do not belong to the program that the DR event was issued for.

- Groups-Participants can belong to a Utility Group. Utility Groups are used as a shorthand way of specifying more than one participant. Specifying a Utility Group is functionally equivalent to specifying each individual participant that belongs to that group.

- DRAS Clients-this is simply an explicit list of the specific DRAS Clients that should receive the DR event.

- Location-Each of the DRAS Clients may have a location attribute that is used to specify where geographically or on the utility or ISO grid that a DRAS Client is located. That location attribute can be used as a means to specify which DRAS Clients should receive a DR event.

Each of the attributes above may be used to define a set of DRAS Clients. For example each participant may have one or more DRAS Clients associated with their account on 
the DRAS. Therefore if a particular ParticipantAccount is specified then it is referring to all the DRAS Clients associated with that account. Since each of the attributes above represent a different criteria for specifying a set of DRAS Clients they can be used in conjunction with each other to specify a specific set of DRAS Clients by logically taking the intersection of all the sets of DRAS Clients that are specified by each attribute.

It should be noted that these propagation rules are a form of business logic within the DRAS and must be implemented by each DRAS that complies with this OpenADR specification. The various parameters and assumptions that the DRAS must follow are shown in Figure 18.

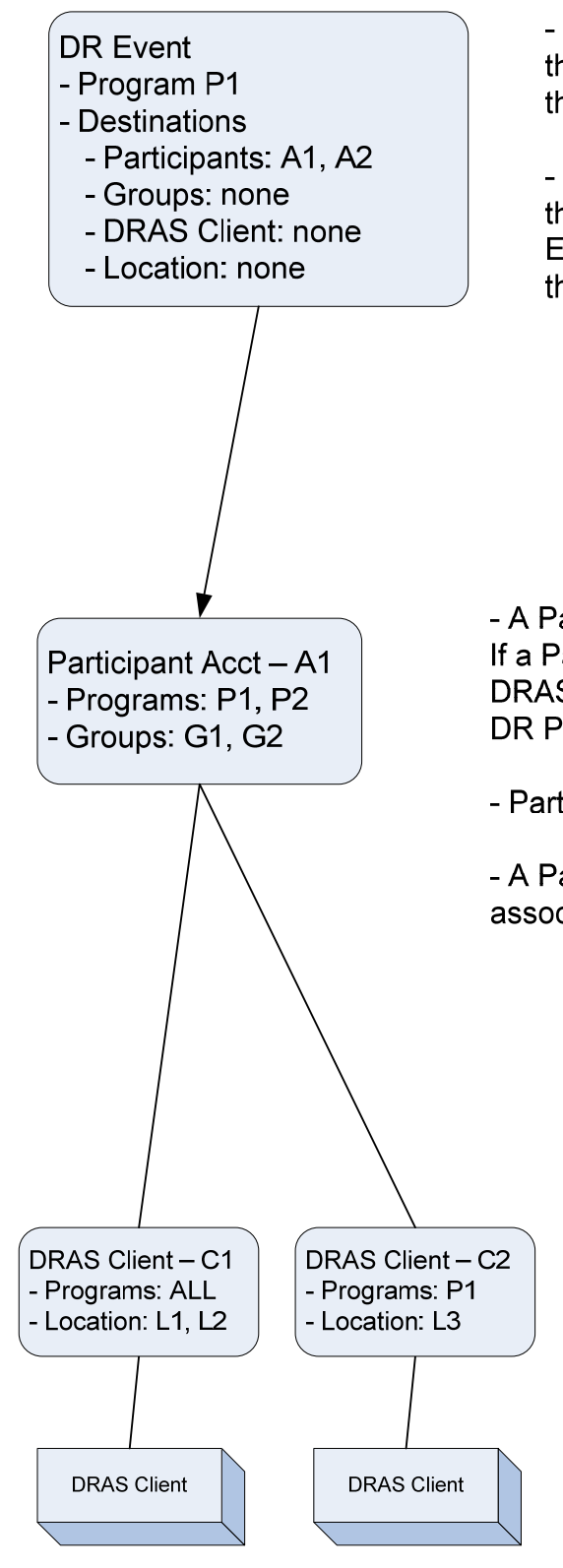

\begin{abstract}
- DR Events are associated with a particular Program. Only those DRAS Clients that belong to that Program will receive that event.
\end{abstract}

- DR Events have a "destination" specification that is used by the DRAS to determine which DRAS Clients receive the DR Event. The destination specification may have one or more of the following attributes:

- List of participants.

- List of Utility groups.

- List of DRAS Clients.

- List of locations.

- A Participant Account may belong to one or more DR Programs. If a Participant Account does not belong to a DR Program then the DRAS Clients associated with it will not receive DR Events for that DR Program.

- Participant Accounts may belong to one or more Groups.

- A Participant Account may have one or more than DRAS Clients associated with it.

- DRAS Clients are associated with one and only one Participant.

- By default a DRAS Client belongs to all the same Programs that the Participant Account that it is associated with belongs to, but it may also belong to a subset of such Programs. If a DRAS Client does not belong to a DR Program then it will not receive DR Events for that DR Program.

- A DRAS Client may have a location specification consisting of one or more of the following attributes: - grid location

- address

- lat/lon coordinates

Figure 18. Relevant Attributes and Structures for Event Propagation 


\subsubsection{DR Event Propagation Examples}

Figures 19 through 26 shows how a DR event must be propagated by the DRAS based upon various destination parameters. Figure 19 provides the sample configuration that will be used as a basis for Figures 20 through 26, which provide examples of how the same configuration may be used to propagate DR events in different ways depending upon the destination specification for a DR event. The bold arrows in each diagram illustrate where the DR event is logically being propagated, including:

- DR Event Propagation for program P2-All Participant Accounts (Figure 20)

- DR Event Propagation for Program P2-Specific Participant Accounts A3, A4 ( Figure 21)

- DR Event Propagation for Program P1-Specific Participant Account A1 ( Figure 22)

- DR Event Propagation for Program P2-Groups G2, G4 ( Figure 23)

- DR Event Propagation for Program P2-Specific DRAS Clients C1, C4, C5 ( Figure 24)

- DR Event Propagation for Program P2-Specific Locations L1, L3 ( Figure 25)

- DR Event Propagation for Program P2-Groups G2 and Participants A4 Specified ( Figure 26) 

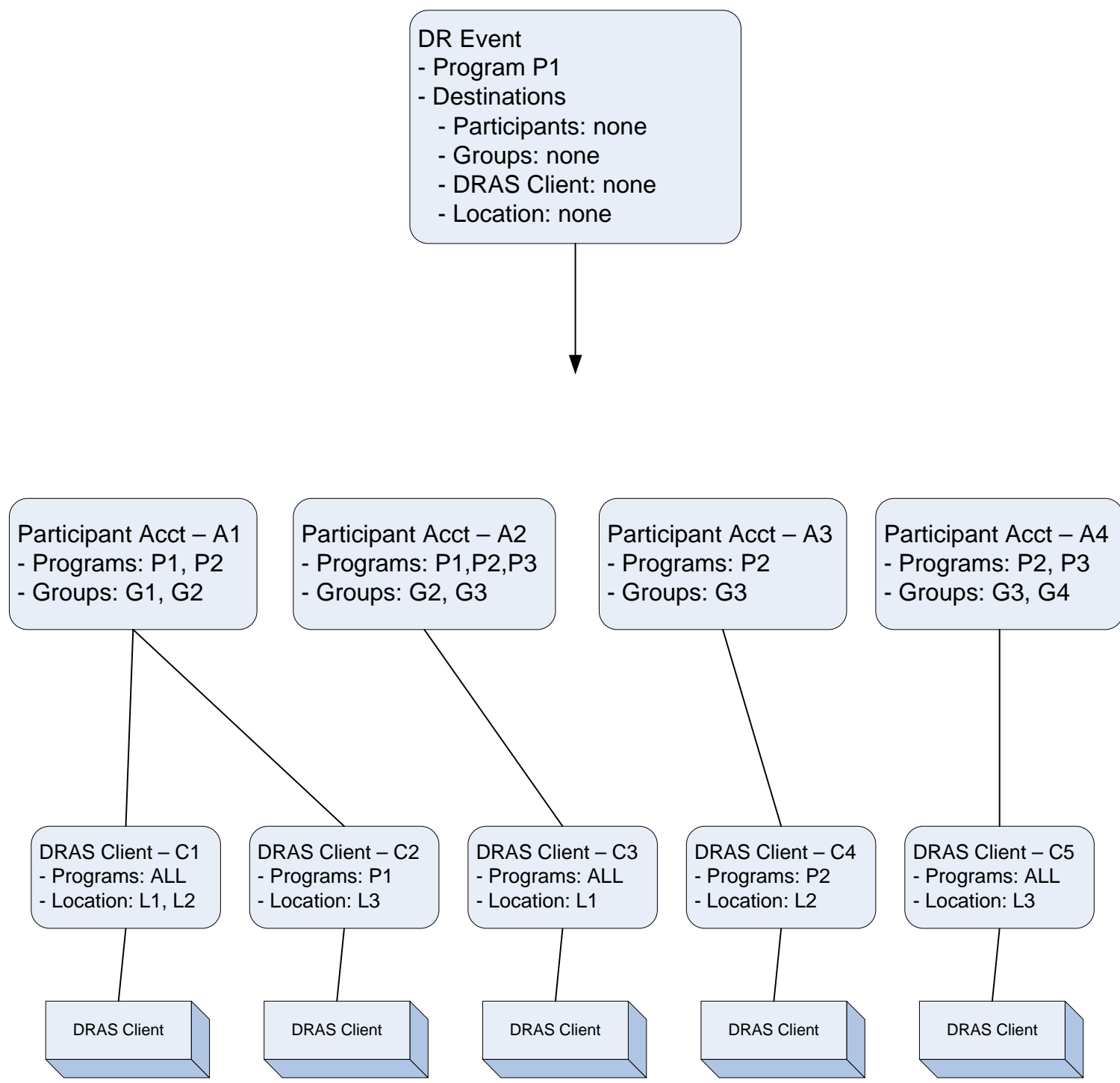

Figure 19. Sample Configuration - Participant Accounts and DRAS Clients 


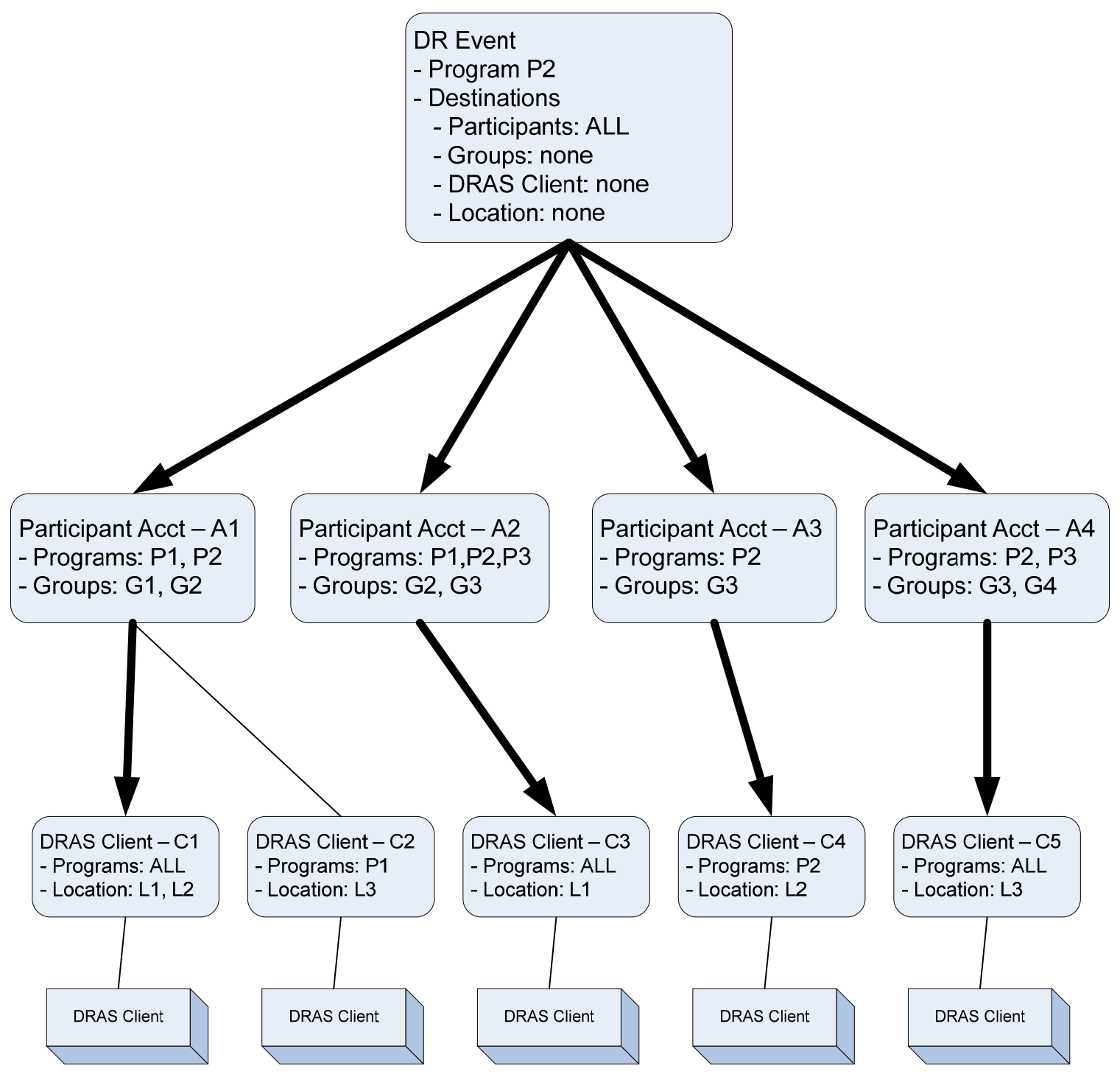

Figure 20. DR Event Propagation for Program P2-All Participant Accounts 


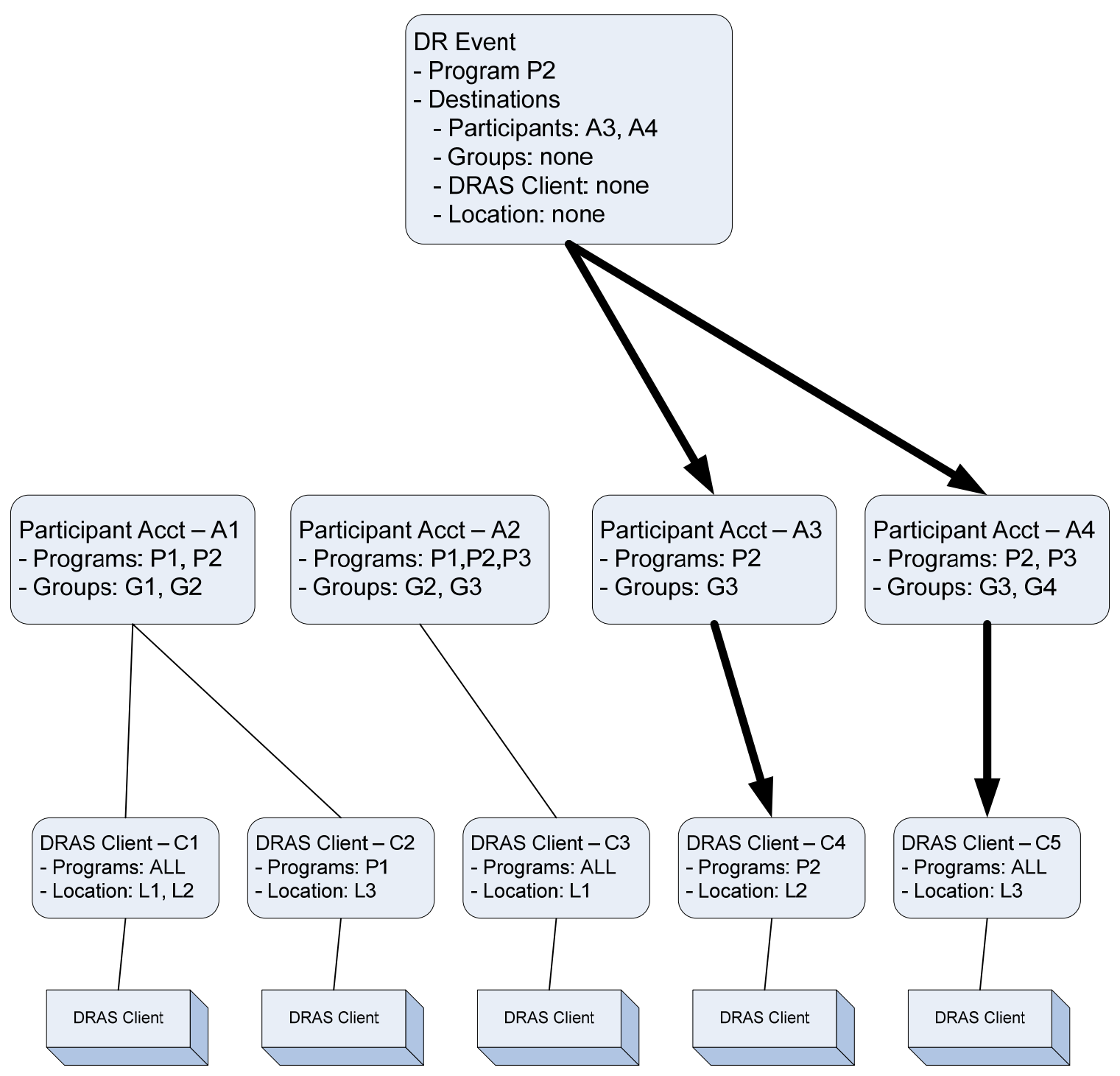

Figure 21. DR Event Propagation for Program P2-Specific Participant Accounts A3, A4 


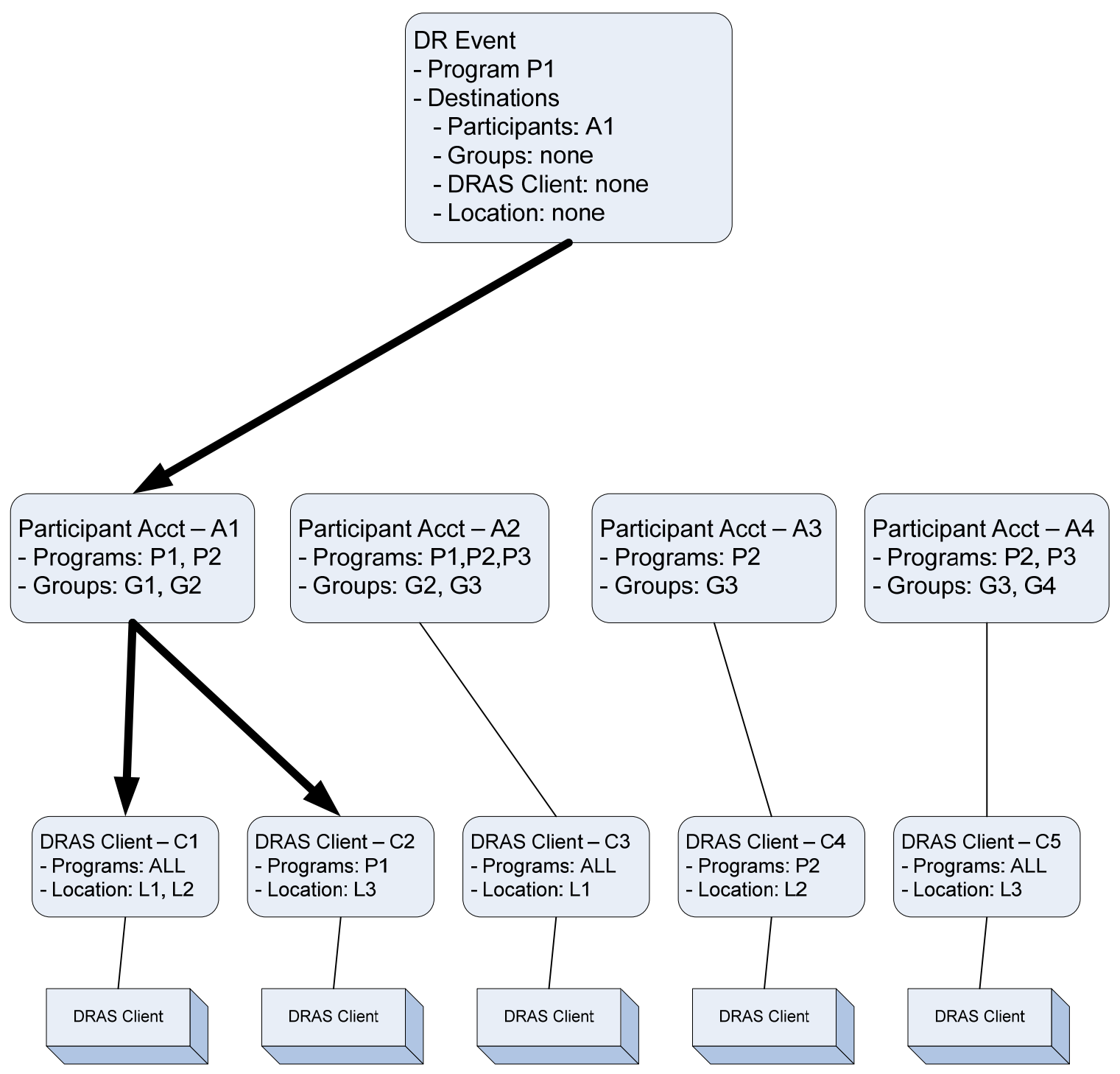

Figure 22. DR Event Propagation for Program P1-Specific Participant Account A1 


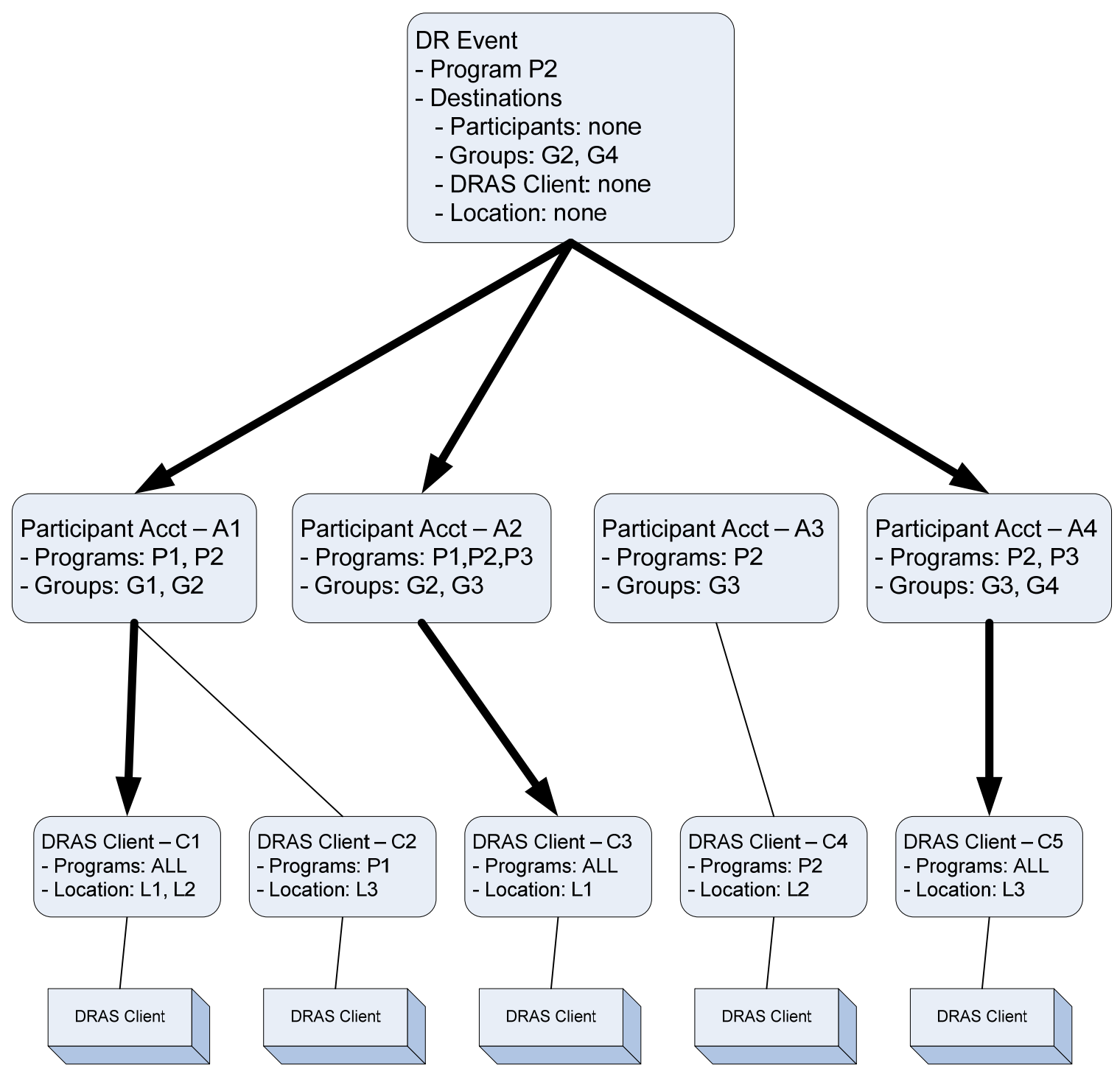

Figure 23. DR Event Propagation for Program P2-Groups G2, G4 


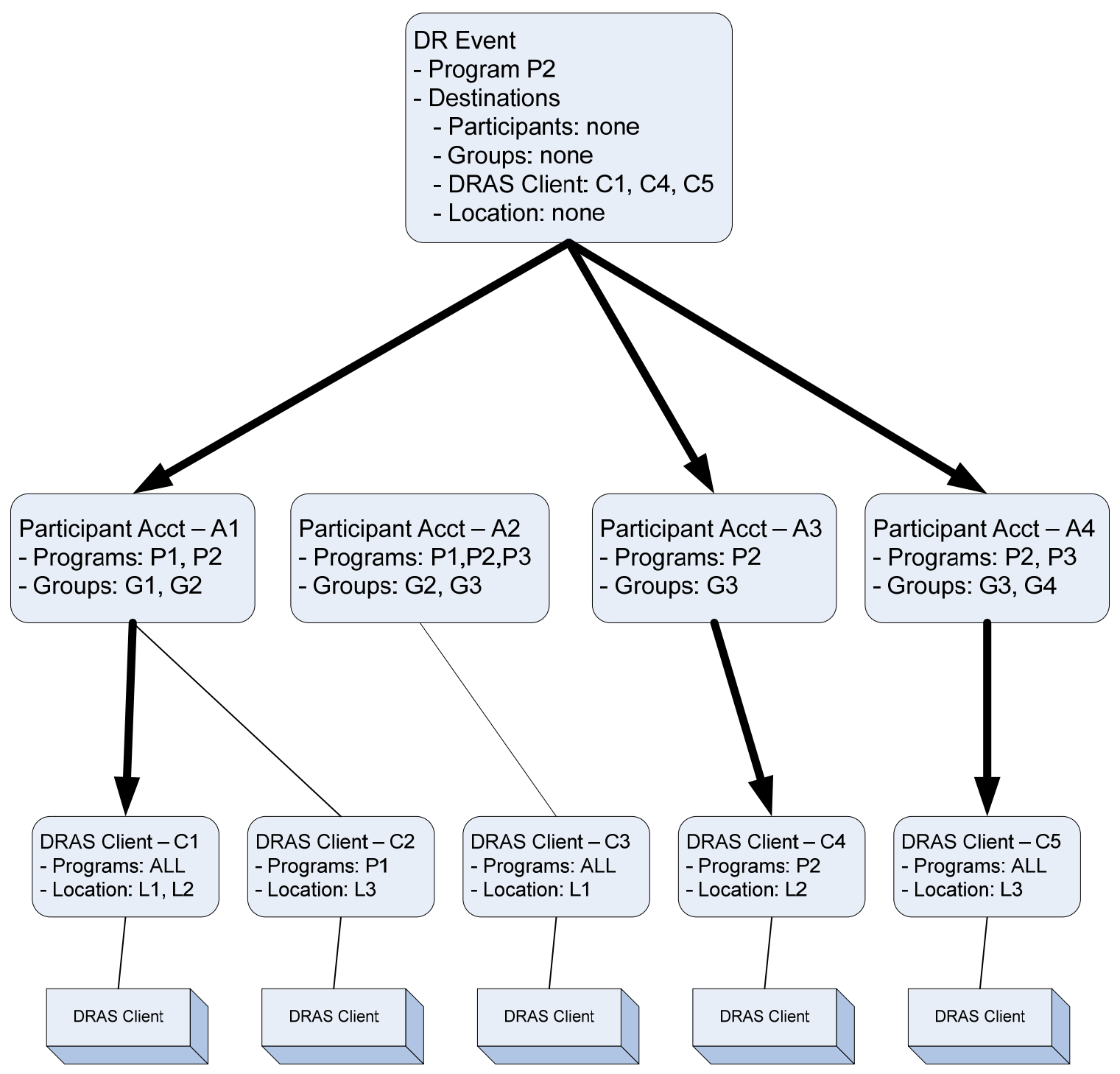

Figure 24. DR Event Propagation for Program P2-Specific DRAS Clients C1, C4, C5 


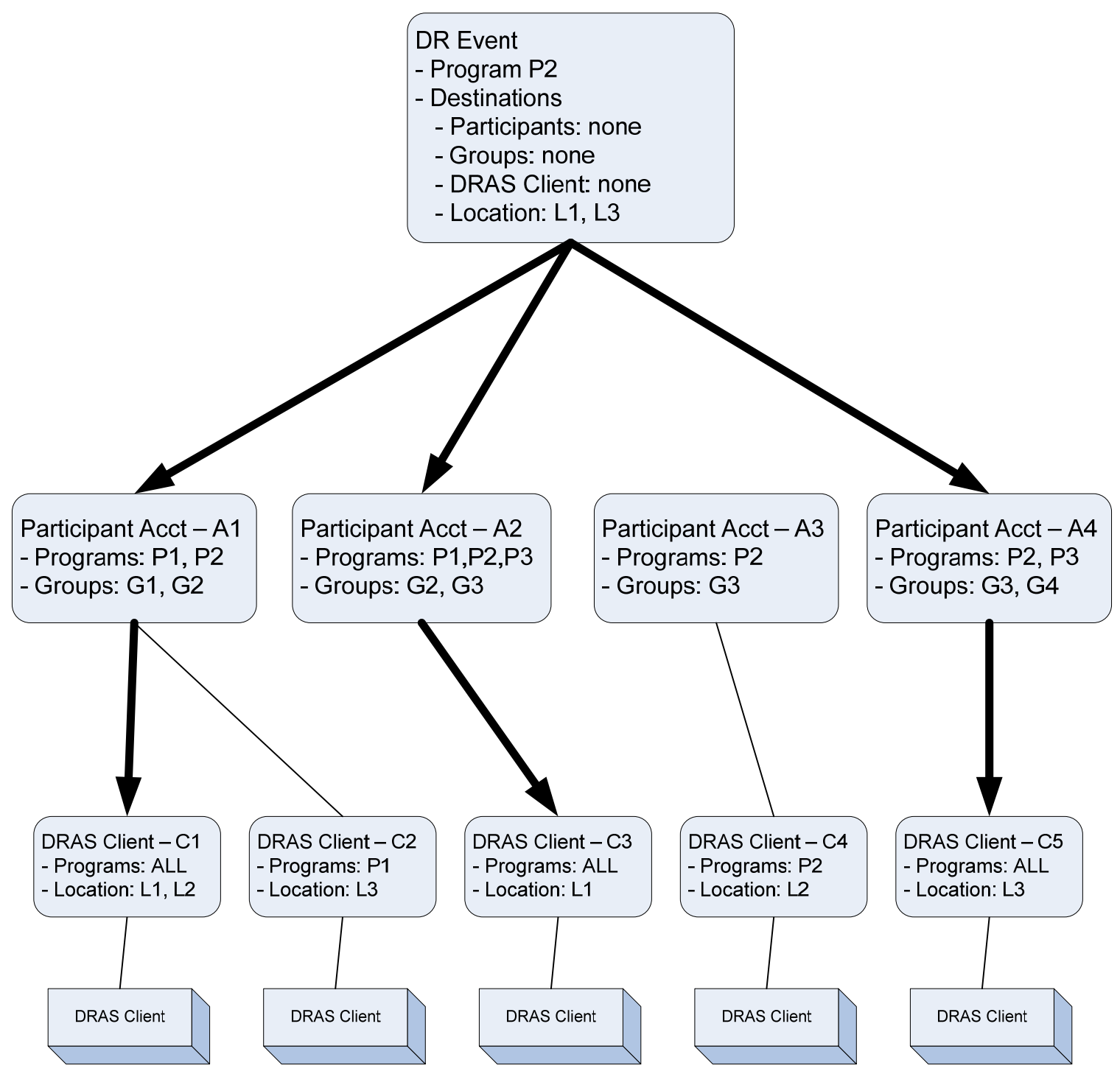

Figure 25. DR Event Propagation for Program P2-Specific Locations L1, L3 


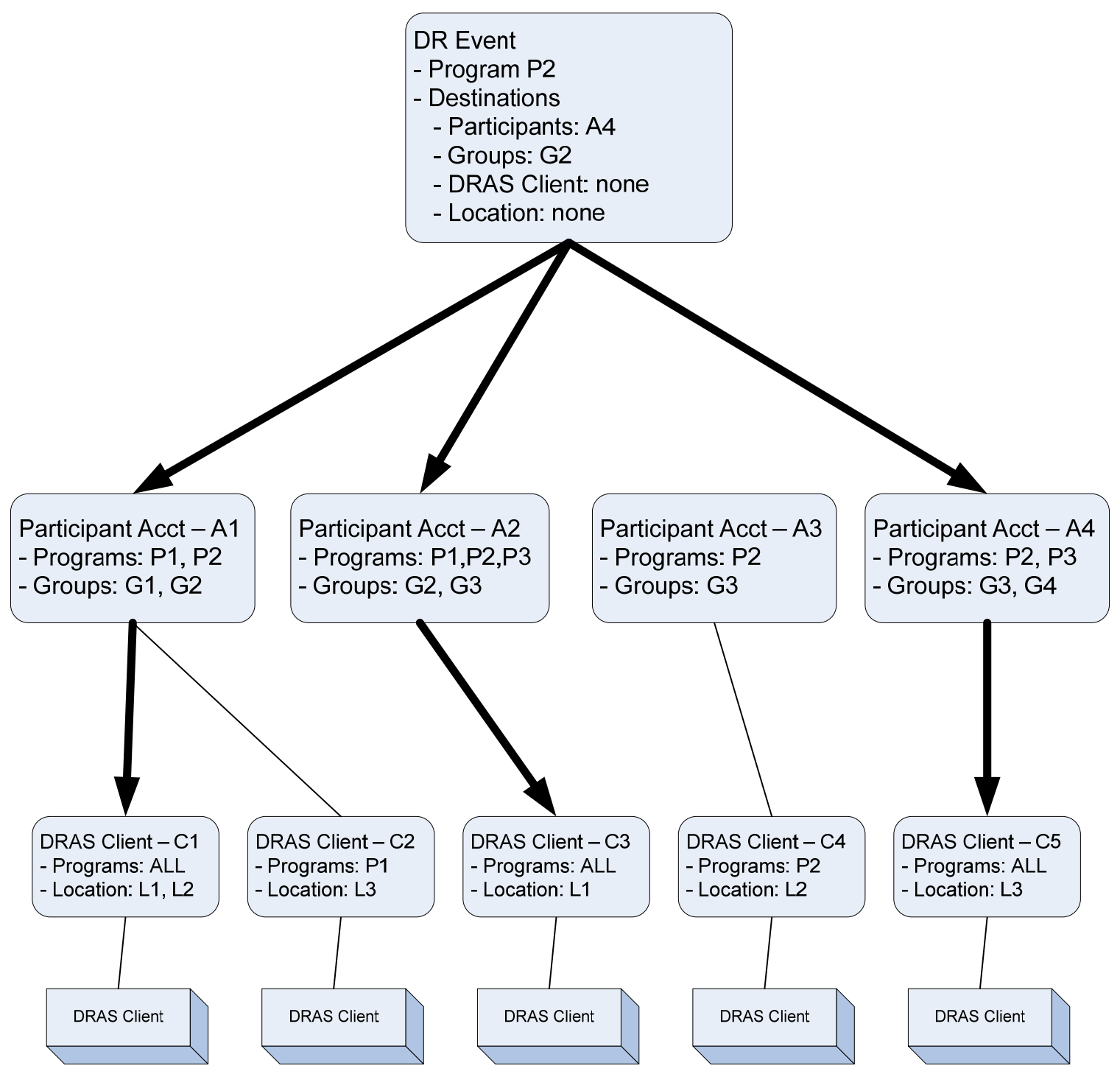

Figure 26. DR Event Propagation for Program P2-Groups G2 and Participants A4 Specified 


\subsubsection{Program Constraints}

This section describes how so called program constraints effect the propagation of DR events. Program constraints are represented by the ProgramConstraint entity. The ProgramConstraint entity represents a set of parameters that constrain various time and date parameters associated with a particular DR event. See Sections 6.4.1.2 and 8.4 for a more detailed discussion of the ProgramConstraint entity. When a DR event is issued the DRAS compares the parameters of the DR event against various sets of program constraints as the DR event is propagated. Figure 27 shows the different points at which ProgramConstraint variables maybe applied to a DR event as it is propagated to a specific DRAS Client.

First the DRAS compares the DR program against ProgramConstraint variables that are configured to be part of the program. In addition to Program Constraint variables associated with a program as a whole it is possible for the utility or ISO to create Program Constraint variables that are applicable to a single DR event. If ProgramConstraint variables exist for a single DR event then they will supersede any ProgramConstraint variables that may exist for the program as a whole. No comparisons are made if a program or a DR event does not have ProgramConstraint variables.

Next the DR event is compared against the ProgramConstraint variables for every participant that is deemed to receive the DR event. Each participant may or may not associate a set of ProgramConstraint variables with their ParticipantAccount that are related to a particular program. If there are no ProgramConstraint variables for the participant and program in question then no comparison is done.

Next the DR event is compared against ProgramConstraint variables for every DRAS Client that is deemed to receive the DR event as described above. Each DRAS Client may or may not associate a set of ProgramConstraint variables that are related to a particular program. If there are no Program Constraint variables for the DRAS Client and program in question then no comparison is done.

The attributes of the ProgramConstraint variables at successive levels must not be in conflict with the attributes at the level above it. What this means is that attributes of the ProgramConstraint variables of a DRAS Client must not be in conflict with attributes of the Program Constraint variables of a participant which are in turn must not be in conflict with attributes of the ResponseSchedule for a program as a whole. Constraints are considered to be in conflict if it is impossible for any DR event to satisfy any of the constraints. 


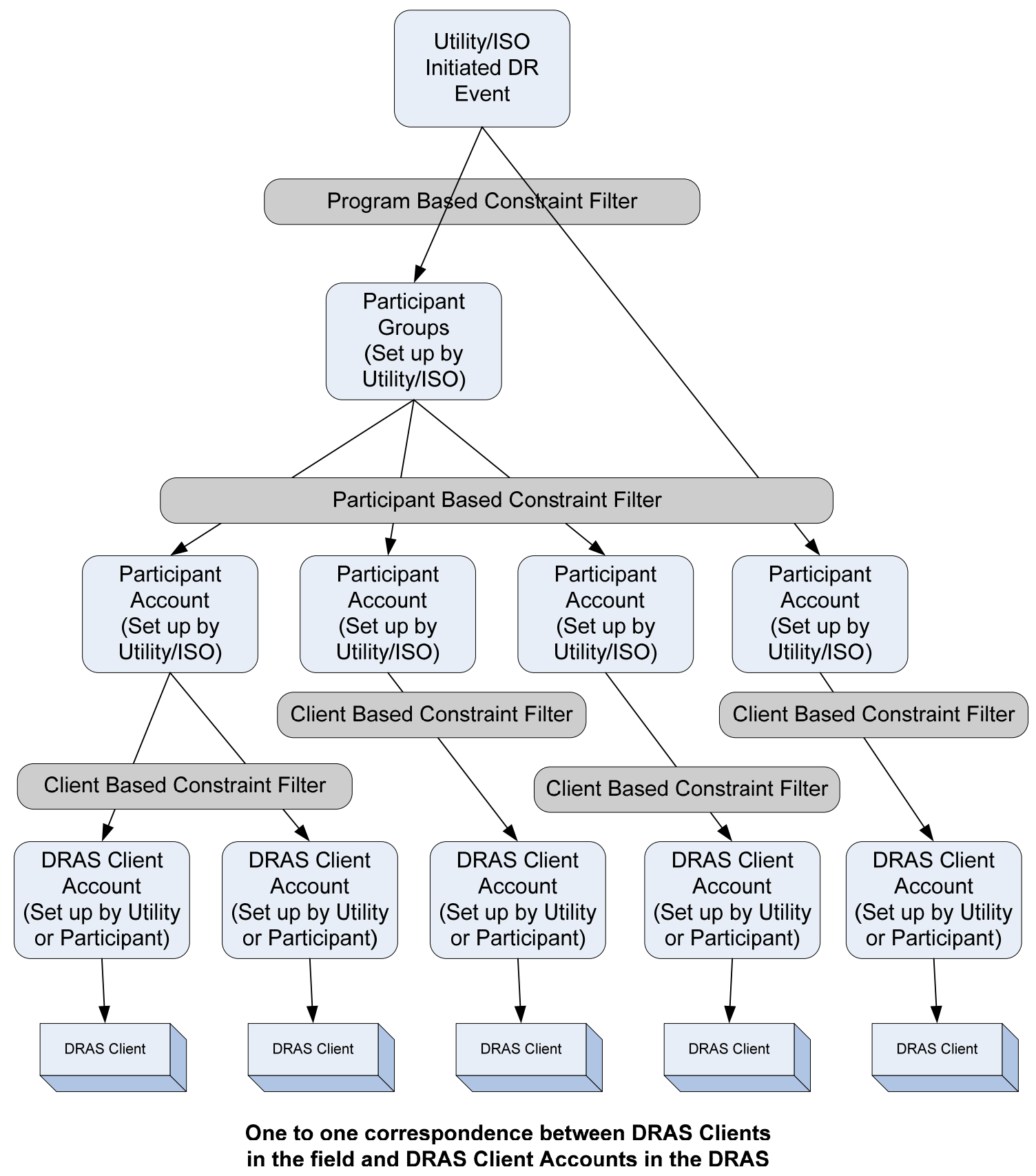

Figure 27. DR Event Constraint Model (Which DRAS Clients Receive the Event) 
Figure 28 shows the comparison process of the DR event as it progresses through the DR event propagation process. The various parameters compared against the issued DR event include:

- Event window-this is the valid time window during the day that a DR event can occur.

- Event duration-this is the maximum event duration for a DR event.

- Notification window-this is the minimum and maximum time before an event that a participant can be notified of a DR event.

- Blackout dates-these are dates during which DR events can not be issued.

- Valid dates-these are the dual of the blackout dates and represent the ONLY dates during which a DR event may be issued.

- Max consecutive days-this is the maximum consecutive days that a DR event can be issued.

Each of these parameters is optional, but if they are defined then they are compared to the following DR event parameters:

- Notification time

- Start time

- End time

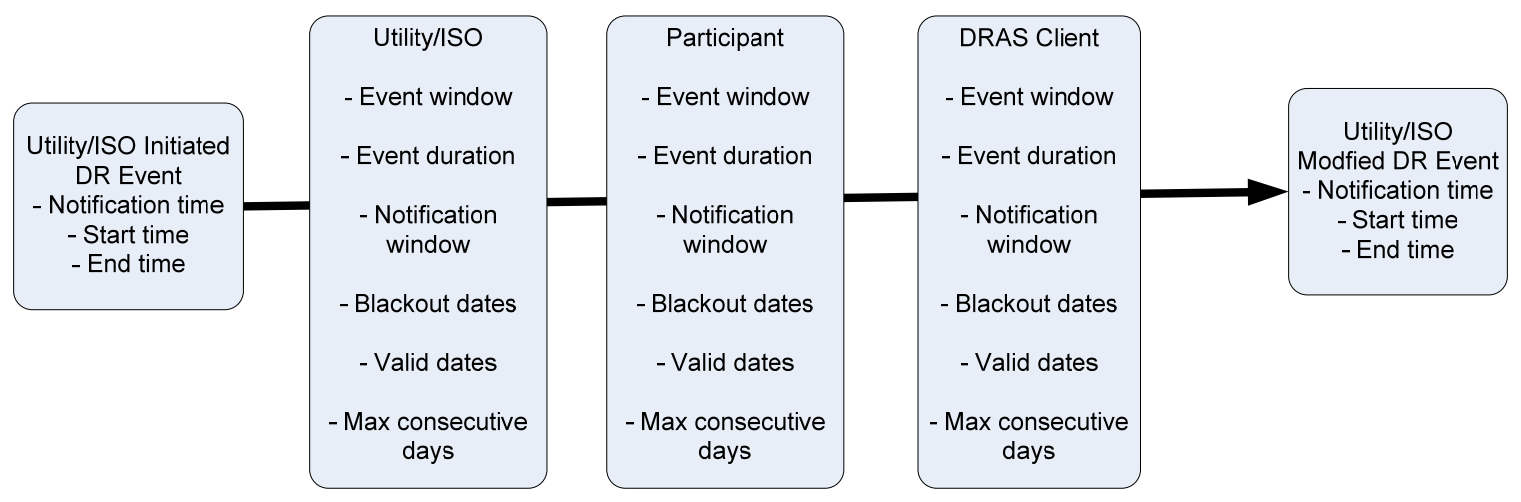

Figure 28. Program Constraints and Filters

The process of comparison checks the parameters of the DR event against the relevant parameters of the ProgramConstraint entity. If there is a mismatch then the DRAS must take an action which is further specified in the Program Constraint entity. Each parameter in the Program Constraint entity has a corresponding attribute which specifies how the DRAS will respond to mismatches between the DR event and the ProgramConstraint entity. The possible actions are the following:

- ACCEPT-simply accept the issued DR event regardless of any conflicts.

- REJECT-reject any DR events that conflict with configured constraints. 
- FORCE-regardless of what the issued DR events parameters are (even if there is no conflict) force them to be the parameters that were configured in the ProgramConstraint entity.

- RESTRICT-modify the DR event parameters so that they legally fall within the bounds of the attributes in the ProgramConstraint entity.

Each of these are referred to as "filter constraints" since they specify how the DR event may be filtered by the DRAS.

Figures 28 through 30 illustrate the various actions taken when there are mismatches between the DR event and the attribute of the Program Constraint variables. Each diagram represents a different schedule related attribute of the DR event and how that specific attribute is handled when there is a conflict between the DR event that is issued and some ProgramConstraint that it is being compared against.

Time of day

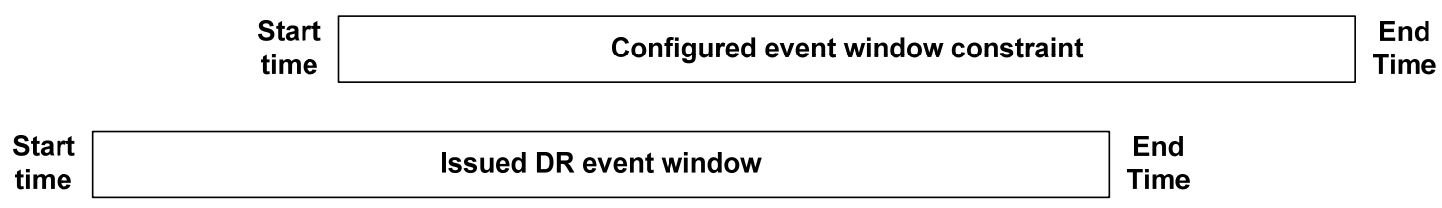

\begin{tabular}{|c|c|c|c|}
\hline \multirow{2}{*}{$\begin{array}{l}\text { Start } \\
\text { time }\end{array}$} & \multicolumn{2}{|c|}{ Filtered DR event window (ACCEPT FILTER) } & $\begin{array}{l}\text { End } \\
\text { Time }\end{array}$ \\
\hline & $\begin{array}{l}\text { Start } \\
\text { time }\end{array}$ & \multicolumn{2}{|c|}{ Filtered DR event window (FORCE FILTER) } \\
\hline & $\begin{array}{l}\text { Start } \\
\text { time }\end{array}$ & Filtered DR event window (RESTRICT FILTER) & $\begin{array}{l}\text { End } \\
\text { Time }\end{array}$ \\
\hline
\end{tabular}

NO DR event issued (REJECT FILTER)

Figure 29. DR Event Window Depending Upon Filter Constraints 


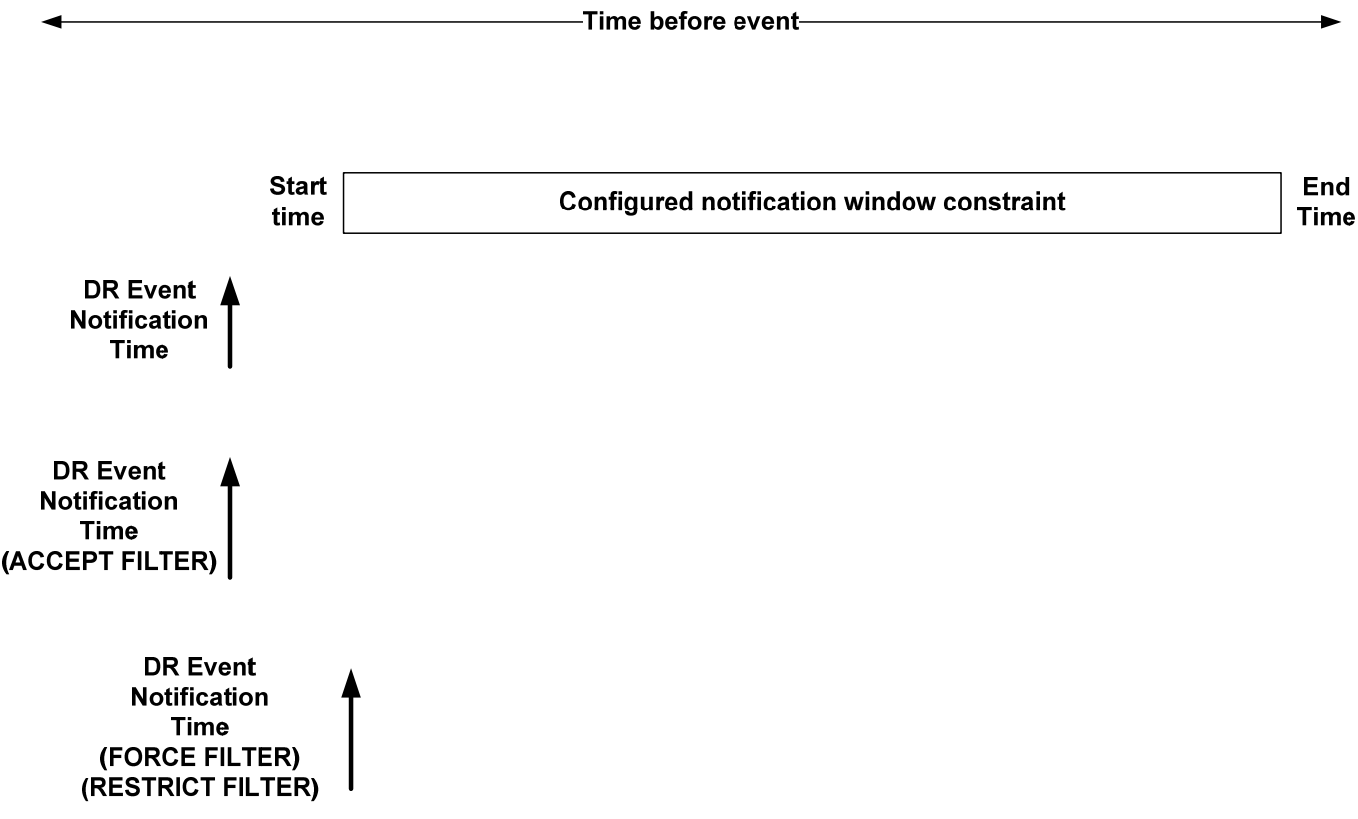

NO DR event issued (REJECT FILTER)

Figure 30. DR Event Notification Time Depending Upon Filter Constraints

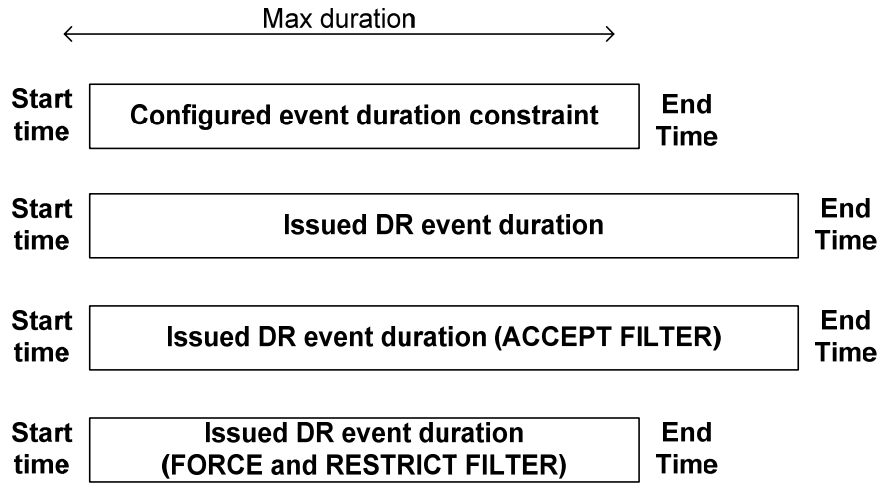

NO DR event issued (REJECT FILTER)

Figure 31. DR Event Duration Depending Upon Duration Constraints

\subsubsection{DRAS Client View of DR Events}

This section presents the DR event model used by the DRAS Client.

When a DR event is issued by the utility or ISO, information concerning the DR event is sent to the various DRAS Clients at the appropriate time depending upon the state of the DR event and the state of the DRAS Client. The type of information sent from the DRAS to the DRAS Client concerning the DR event includes the following: 
- DRAS Client identifier

- Program name associated with the DR event

- Identifier for the DR event that was issued by the utility or ISO

- Transaction identifier for the DR event message. Used to identify specific DR event messages.

- Flag to signify if the DR event is a test message

- Flag to signify whether the DRAS Client is on or off line

- Simple DRAS Client data-this data is intended to be used by Simple DRAS Clients

o Event status

o Operation mode value

o Current time

o Operation mode schedule

- Smart DRAS Client data-this information is intended to be used by Smart DRAS Clients.

o Event notification time

o Event start time

o Event end time

o Event information (EventInfo instances)

- Custom data-this is a placeholder for information that is intended to be used for purposes beyond the scope of this document. It may be used to send proprietary commands or information to a DRAS Client.

This collection of information is represented by the EventState entity and represents the state that a DRAS Client is in with respect to a particular DR event. See Section 8.12 for a more detailed description of the EventState data model and schema.

This rest of this section describes in more detail how the EventState entity is exchanged with the DRAS Client.

\subsubsection{Modes of Interaction (PUSH versus PULL)}

The DRAS and the DRAS Client may exchange EventState information using two different modes of interaction - PUSH and PULL.

In the PUSH mode ( Figure 32), the EventState information is "pushed" from the DRAS to the DRAS Client. This means that the communication of the EventState information is initiated by the DRAS. In terms of Web services this means that that DRAS Client is the Web server and the DRAS is the Web client. 


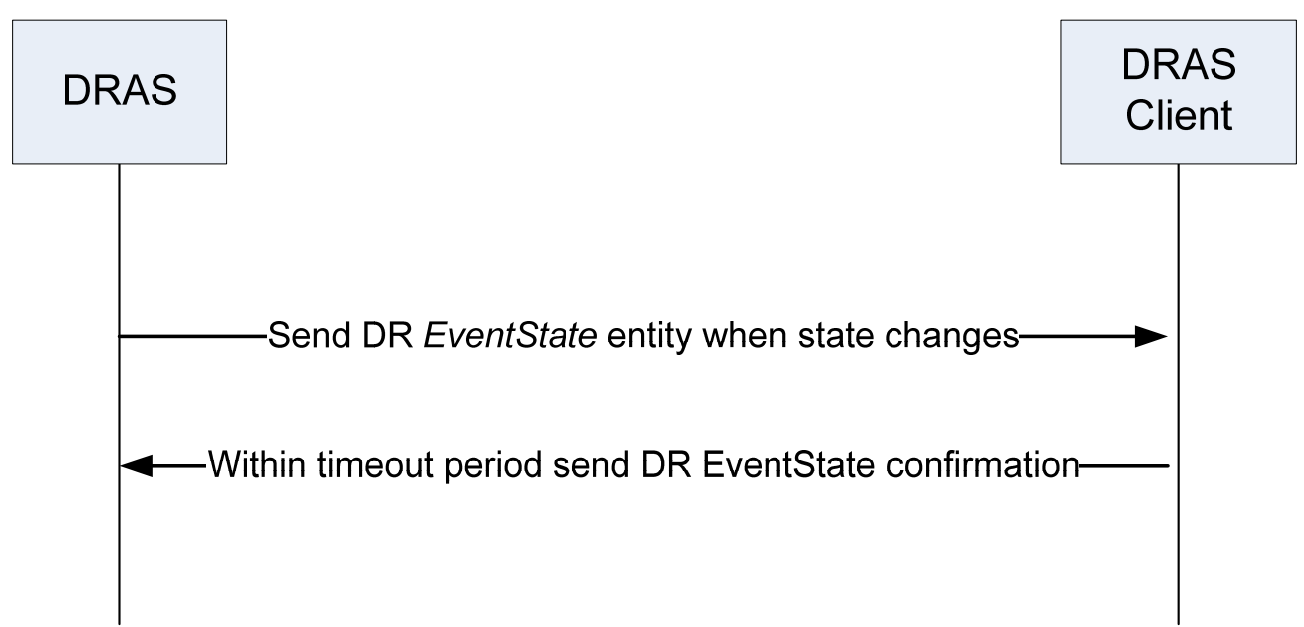

Figure 32. PUSH Model Sequence Diagram

Note that in the PUSH model of information exchange the EventState is transmitted to the DRAS Client whenever the state of the DR event that is being monitored by the DRAS changes. The various states of the EventState are discussed more detail below.

In the PULL mode ( Figure 33), the EventState information is "pulled" from the DRAS by the DRAS Client. This means that the communication of the EventState information is initiated by the DRAS Client. In other words the DRAS Client polls the DRAS for the EventState information. In terms of Web services this means that the DRAS is the Web server and the DRAS Client is the Web client. In the PULL model of information exchange the EventState may be requested by the DRAS Client at any time.

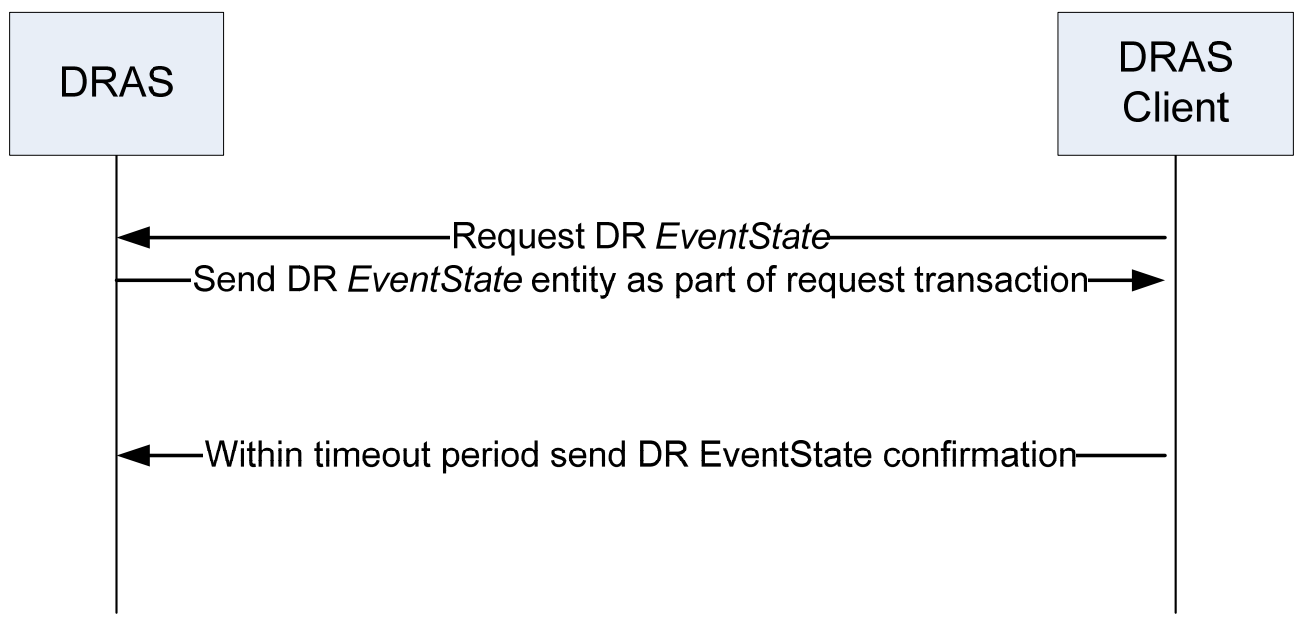

Figure 33. PULL Model Sequence Diagram

Note that in both cases the DRAS Client sends a confirmation message to the DRAS upon receipt of the EventState information. This confirmation message is intended to give an additional level of confirmation beyond the reliable communications used for the Web services interface and to allow the DRAS Client to give additional information 
related to how it intends to respond to the DR event. More information concerning the details of the confirmation message data model can be found in 8.12.

The DRAS must use the EventStateConfimation message as a means to confirm that the DRAS Client has received the EventState information. If an EventStateConfirmation message is not received within some time out period then the DRAS must assume that the EventState message was not received by the DRAS Client.

The DRAS must support two-way communications for both the PUSH and the PULL model of interaction, but the DRAS Client is only required to support one or the other. Typically the PULL model may be used since the DRAS Client has more control over the communications including the ability to more easily communicate through firewalls and being network-friendly. The PUSH method would typically be used in scenarios where very low latency of the messages delivery is required when a state change occurs. The specific situation of implementing PUSH versus PULL models is outside the scope of this document.

\subsubsection{Simple Versus Smart DRAS Clients}

The DRAS supports two different types of DRAS Clients-Simple and Smart.

The Smart DRAS Client is assumed to be capable of dealing with all the EventInfo information that may be associated with a DR event that is initiated by the utility or ISO. It can parse all the EventInfo information and make decisions about how to respond to that particular DR event information.

On the other hand there are many cases where a Simple DRAS client is needed. These cases have simplified EMCS that are incapable of any sophisticated logic or the ability to deal with the wide range of information types that may be associated with a DR event. In these cases, the DRAS translates the EventInfo information associated with a DR event into a much simpler form, known as a Simple DRAS Client.

The DRAS must be capable of dealing with both Smart and Simple DRAS Clients. Details on both of these scenarios are described below.

\subsubsection{DR Event Information}

DR programs are typically designed to use a variety of information to cause reactions by participants to DR events that are issued by the utility or ISO. In some cases prices are used to trigger responses to the DR events while in other cases it might be a shed or shift level. In general there can be a wide range of different types of information associated with a DR event depending upon how the DR program was designed. Therefore the data models used to describe the information associated with DR events are designed to accommodate the wide range of information that may be associated with a DR event. This information is represented by EventInfoInstance entities.

When a program is defined within the DRAS there are specifications associated with the program that define what type of information may be associated with a DR event when 
one is issued for that program. Each type specification for an EventInfoInstance is referred to as an EventInfoType. A program may be defined that allows for multiple different types to be associated with a program. Each EventInfoType contains the following attributes and elements:

\section{EventInfoType}

- name-this is the name of the type. Analogous to a variable name.

- typeID-this identifies the type of information and may take on one of the following values:

o PRICE_ABSOLUTE - Price number, i.e. $\$ 0.25$

o PRICE_RELATIVE - Change in price, i.e. $-\$ 0.05$

o PRICE_MULTIPLE - Multiple of current price, i.e. 1.5

o LOAD_LEVEL - Amount of load based on an enumeration, i.e. moderate, high, etc.

o LOAD_AMOUNT - Fixed amount of load to shed or shift, i.e. $5 \mathrm{MW}$

o LOAD_PERCENTAGE - Percentage of load to shed or shift, i.e. 10\%

o GRID_RELIABILITY - Number signifying the reliability of the grid

- $\quad$ scheduleType - This specifies how a schedule may be associated with the DR Event information is defined and may take on the following values:

o NONE-there is no schedule and thus the EventInfo does not change values during the entire DR event ACTIVE state.

o DYNAMIC-The time schedule is not fixed during configuration, but can be set when the DR event is issued.

o STATIC-The schedule is fixed when the DR program is configured within the DRAS

- $\quad$ schedule-If the scheduleType is STATIC then this is the configured schedule. A schedule is a sequence of time slots that are valid over the entire ACTIVE period of a DR event. Each time slot may take on a different value in the EventInfoInstance

- enumerations - This is a list that defines a fixed set of values that the EventInfo instance may take. If defined, the EventInfoInstance is an enumeration and can take on any of the values in the list. If left undefined, the EventInfo instance can take on any contiguous value between the minValue and maxValue.

- minValue-minimum possible value of an EventInfoInstance.

- maxValue-maximum possible value of an EventInfoInstance.

Note that when a DR event is issued, the EventInfoInstance variables that are associated with the DR event may take on values that change according to some schedule during the ACTIVE state of the DR event. Also note that the schedule that defines when these values change may be defined as part of the definition of an EventInfoType or it may be 
defined when the DR event is issued. See Section 8.9 for a detailed description of the EventInfoType schema.

Note that the DRAS Client never sees the EventInfoType definitions within the DRAS. Nonetheless it is useful to understand their structure in order to understand how the DRAS translates information from the various EventInfoInstance variables described below into the simple levels used by a Simple DRAS Client.

The EventInfoType defines the type of information that is associated with a DR event and are specified as part of a program. Thus when a DR event is actually initiated it contains instances of the EventInfoType that were defined to belong to the program. These instances are referred to as an EventInfoInstance and from the DRAS Clients point of view are defined as follows.

\section{EventInfoInstance}

- eventInfoName-this is the same as the name field in the corresponding EventInfoType definition.

- eventInfoTypeID-This is the type identifier of the data and takes on the value defined in the typeID of the EventInfoType definition.

- eventInfoValues-These are the actual values of the instances. There may be more than one if there is a schedule of values.

See Section 8.12 for a detailed description of the EventInfoInstance schema, especially as it applies to how the schedule of values is defined.

\subsection{Smart DRAS Client Event Information}

The EventState message sent to a DRAS Client contains the following set of fields:

- Smart DRAS Client type data-this information is intended to be used by Smart DRAS Client

o Event notification time

o Event start time

o Event end time

o Event information (EventInfoInstance)

This information is derived directly from the DR event (UtilityDREvent) that was issued by the utility or ISO. The "event information" field is simply the collection of

EventInfoInstance data as described above. In general the event timing (notification, start, and end times) is the same as what was specified in the DR event when it was issued by the utility or ISO, but there may be exceptions if there were ProgramConstraint variables defined within the DRAS that caused these values to be altered. For more information on Program Constraint see Section 6.5.2.2.

It is assumed that a Smart DRAS Client is capable of parsing and dealing with the various fields defined above. 


\subsection{Simple DRAS Client Event Information}

It is assumed that a Simple DRAS Client type is not capable of dealing with the sophistication of the information that a Smart DRAS Client is. Therefore the EventState information for a participant with a Simple DRAS Client is a simplified derivation of the information sent by the utility or ISO when the DR event is initiated. In this case there are the following two state variables that describe the DR event state:

- Operation Mode: This depicts the operational state of the facility and can take on the following values:

(1) NORMAL operation

(2) MODERATE shed or shift

(3) HIGH shed or shift

(4) SPECIAL

- Event Status: This depicts the current temporal state of a DR event and can take on the following values:

(1) NONE-no event pending

(2) FAR-event pending far into the future.

(3) NEAR-event pending soon.

(4) NOW-event currently in process.

In general, the Event status variable always transitions from NONE to FAR to NEAR to NOW. The transition from FAR to NEAR is a configurable parameter of a program and in fact there may not even be a NEAR state in which case the FAR value could simply be interpreted as meaning "Event Pending".

The Operation Mode variable takes on values according to a schedule during the event that is defined by the participant or the utility or ISO. This schedule is specified by using a set of rules that determine how the EventInfoInstance of the UtilityDREvent is translated into one of the simple values of the operation mode. Since the participant is free to schedule how the Operation Mode variable changes, this defines a so called "Response Schedule" for how that participant responds to DR events. The response schedule is represented by the ResponseSchedule entity.

A ResponseSchedule is an ordered list of rules represented by the OperationStateSpec entity. An OperationStateSpec within a ResponseSchedule represents a set of rules that are valid within a specific time slot of the ACTIVE period of the DR event. These rules dictate how the Operation Mode variable will transition during the time slot of the OperationStateSpec The time slots that define the different OperationStateSpec entities form points at which the Operation Mode values may transition, but the Operation Mode values may also transition at times in the middle of the time slot associated with an OperationStateSpec if the value associated with an EventInfoInstance happens to change then. 
The rules within an OperationStateSpec are expressed in terms of a table wherein each row in the table represents a Boolean equation such that if the equation is true then the corresponding Operation Mode value will be set. The equations are Boolean comparisons of the existing EventInfoType names for this program. Table 3 is an example table for a single OperationStateSpec entity where there are two EventInfoTypes with the names of RTP and BID that were defined for the program.

Table 3 OperationStateSpec Entity

\begin{tabular}{|l|l|}
\hline Value & Equation \\
\hline MODERATE SHED OR SHIFT & RTP $>5$ \&ID $>10$ \\
\hline HIGH SHED OR SHIFT & RTP $>10$ \& BID $>10$ \\
\hline MODERATE SHED OR SHIFT & RTP $>5 \&$ BID $<5$ \\
\hline SPECIAL & RTP $>15$ \\
\hline NORMAL OPERATION & TRUE \\
\hline
\end{tabular}

Note that each row in the table is evaluated from top to bottom until one of the equations is true. Whichever equation is true then the corresponding value is used to set the operation mode value. Note that if none of the rows are true then the operation mode value does not change. It is therefore good practice to put a default TRUE value at the very end which will be used if none of the other equations are true.

The following Boolean operations should be supported:

- AND

- OR

- $\mathrm{XOR}$

- NOT

- GREATER THAN, GREATER THAN OR EQUAL

- $\quad$ LESS THAN, LESS THAN OR EQUAL

- $\quad$ EQUAL, NOT EQUAL

- GROUPING, i.e. parenthesis

In general the equation can be represented as a simple string. It is beyond the scope of this document to define the exact syntax of the rules strings.

Figure 34 shows a number of different Operation Mode transitions during the ACTIVE period of the DR event. These transitions of the Operation Mode value could be caused either by the EventInfoInstance of the DR event changing values or by the transition from one OperationStateSpec to another within a ResponseSchedule. 


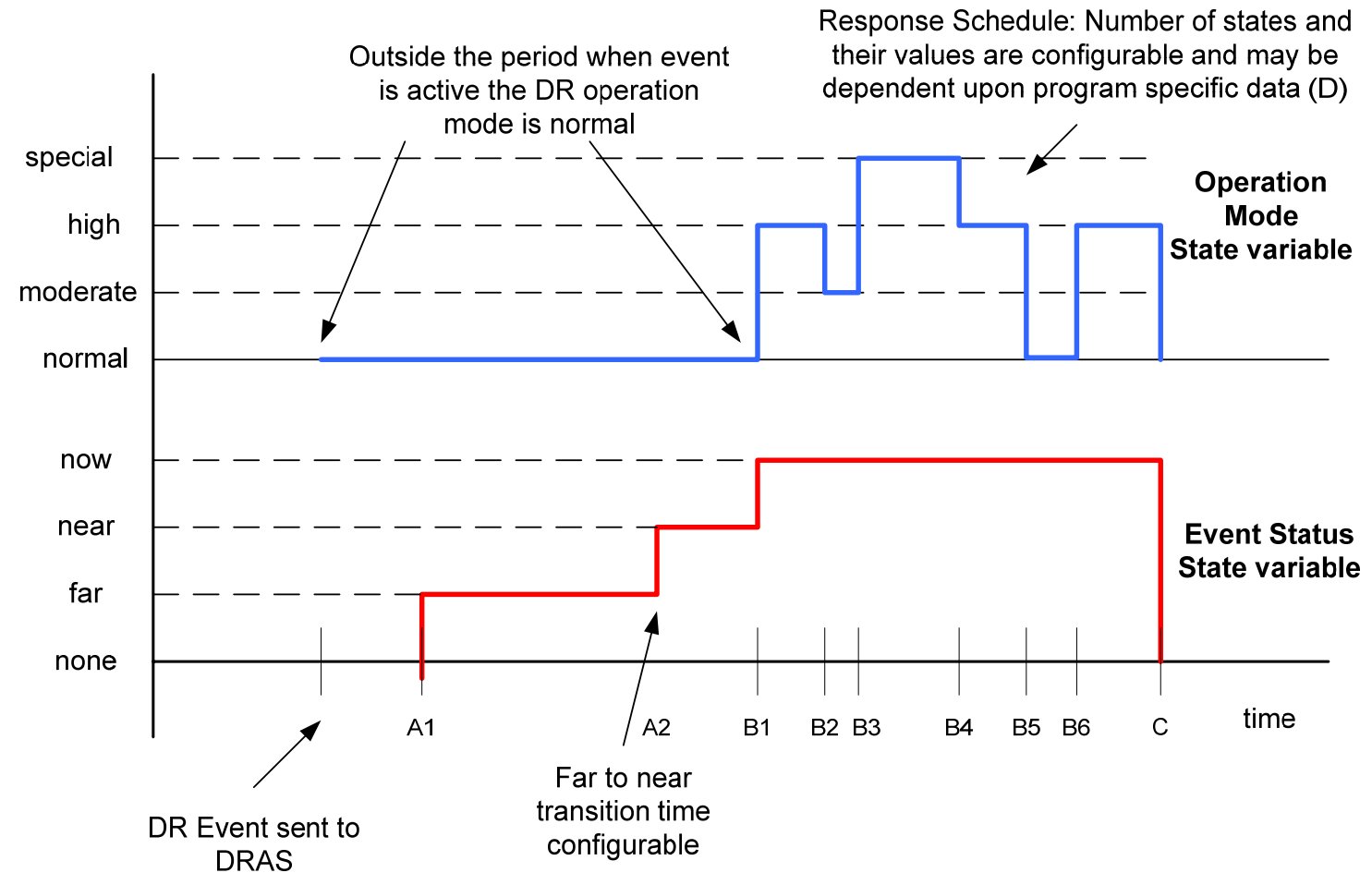

Figure 34. DR Event State Model (Simple Client View)

\subsubsection{DR Event States}

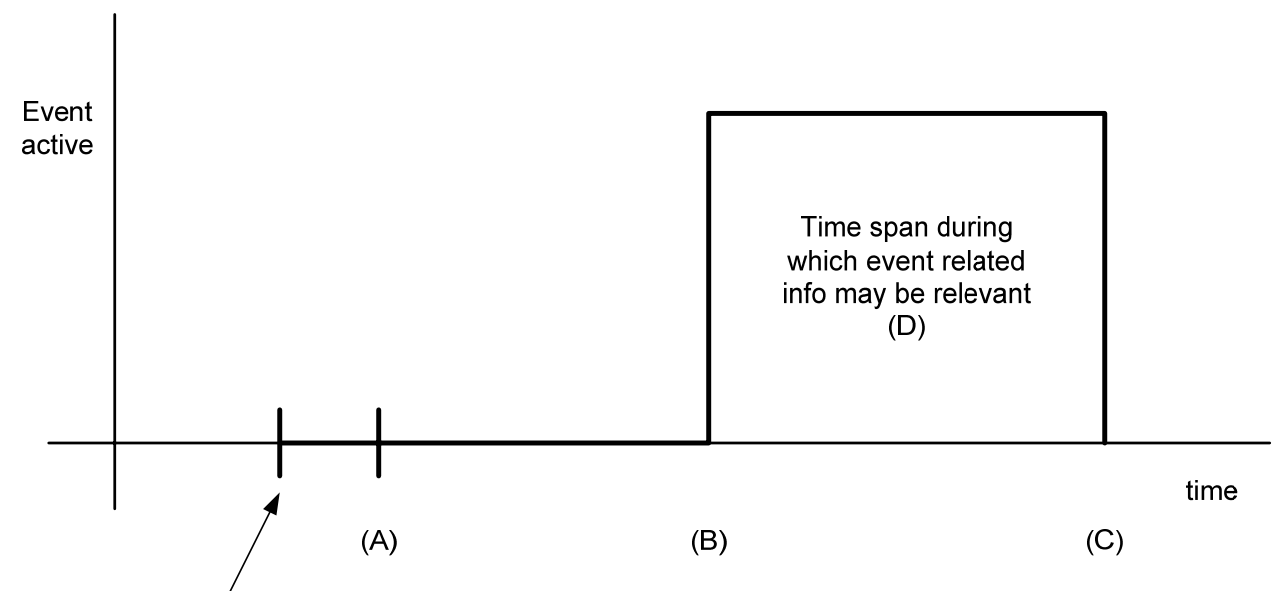

DR Event sent to

DRAS

\section{Figure 35. DR Event Model (Utility or ISO View)}

For reference purposes, the DR Event Model (Figure 16) is repeated here as Figure 35. From the DRAS point of view a DRAS Client may be in one of the following modes of operation:

- opt-in-DR events are handled and sent to the DRAS Client as they normally would be. 
- opt-out-The DRAS Client has opted out from receiving DR event information and none will be sent when they are in this state.

- test-This is used for test purposes and is analogous to the DRAS Client being off line. No DR events will be sent automatically from the DRAS, but a DRAS installer may send test messages to the DRAS Client.

Note that the states listed above are all from the point of view of the DRAS and the DRAS Client responds to the state the DRAS is in. It is the DRAS that changes its behavior and where the logic resides in relation to the DRAS Client.

The DRAS is responsible for tracking the event states for each of the DRAS Clients in order to send the DR event information to the DRAS Client at the appropriate time. From the DRAS Client's point of view there is a so-called DR event state the DRAS Clients are in which is represented by the EventState entity. Normally a DRAS Client's event state is "IDLE" meaning that there are currently no active or pending DR events. This changes when the utility or ISO initiates a DR event in the DRAS. The DRAS tracks the DR event state for each DRAS Client and can provide the current state information at any time for that DRAS Client. It can be in different states, depending upon whether the participant uses a Smart DRAS Client or a Simple DRAS Client.

Figure 36 is the state transition diagram for a general DR event. For a Smart DRAS Client these are the only states that are relevant, but for a Simple DRAS Client there may be sub states during the ACTIVE period which each represent the various level transitions of the Operation Mode variable.

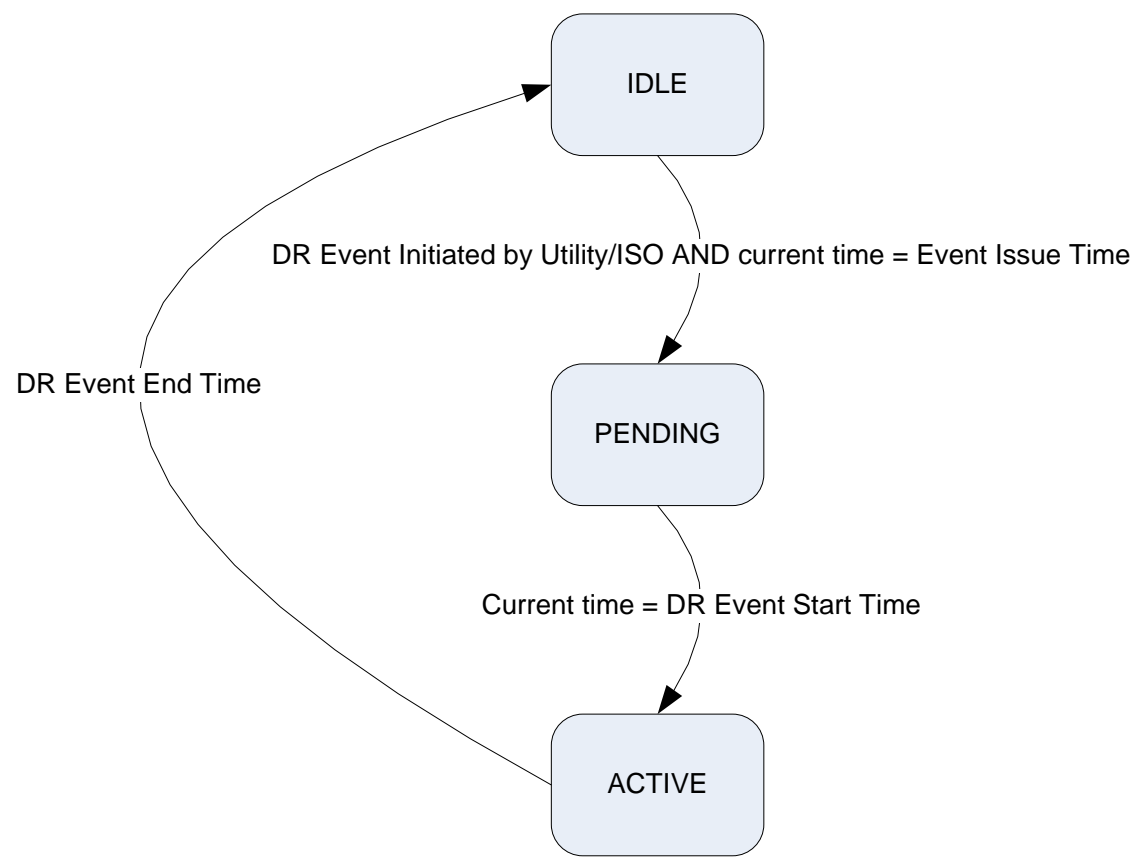

Figure 36. Transition Diagram for a General DR Event 
Figure 37 shows the example state transition diagram that corresponds to Figure 36. Note that the large states correspond directly to Figure 36 and are the only states that exist for a Smart DRAS Client, whereas the sub-states within the ACTIVE state are additional states that may exist for a Simple DRAS Client.

The notion of states are important because the transition times from one state to another define the times when the EventState entity is sent from the DRAS to the DRAS Client in PUSH mode. In PULL mode the DRAS Client polls the DRAS and it simply responds with the appropriate EventState that corresponds to the time when the poll was made.

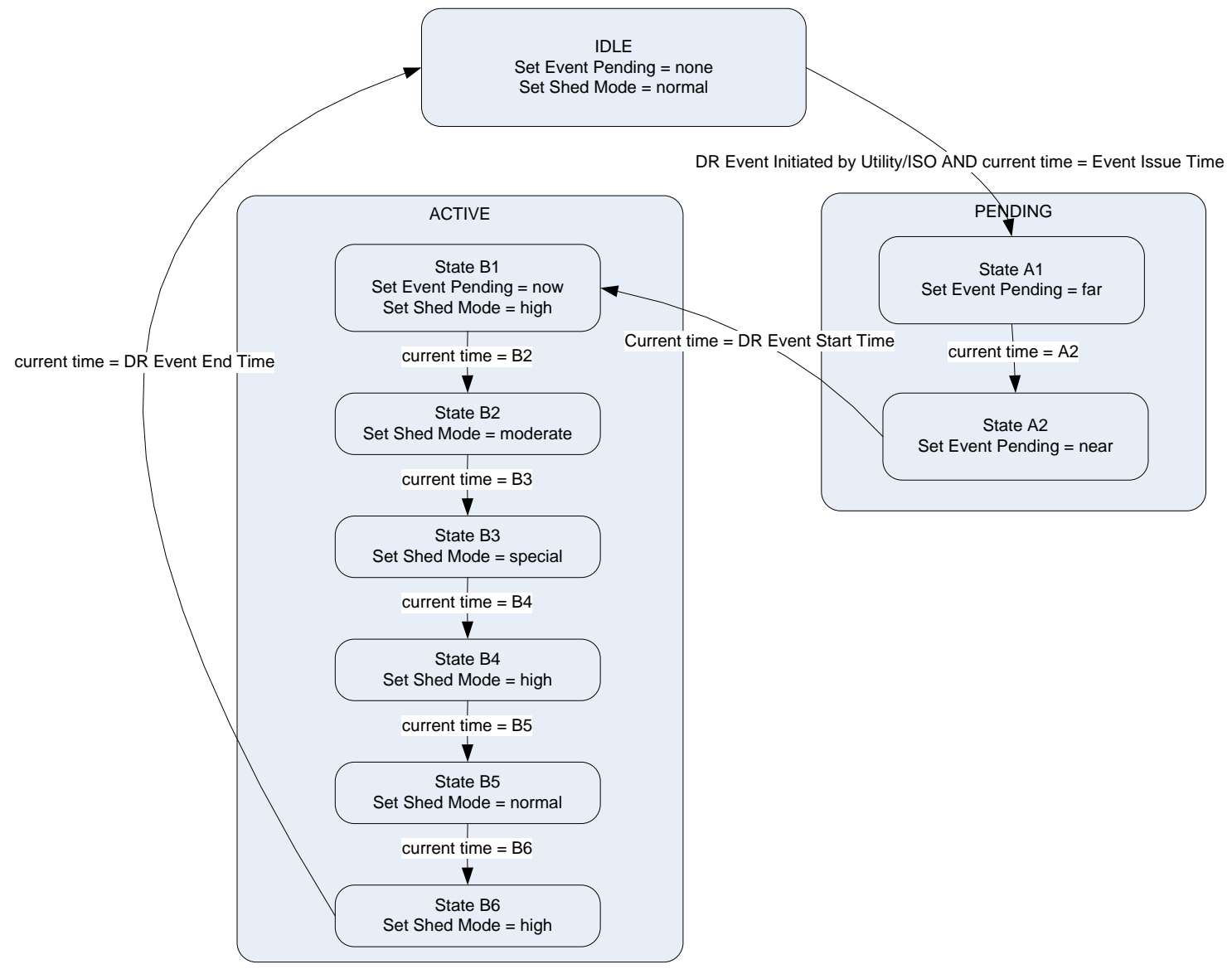

Figure 37. DRAS Client DR Event State Simple DRAS Client State Transition Diagram

\subsection{DR Automated Bidding Models}

The DRAS may support automated bidding by participants into DR programs by supporting the concept of a "standing bid" for that participant. A standing bid is a bid that will be submitted by the DRAS for a participant if no other bid is submitted by the participant. The ability to automatically submit standing bids increases the level of participation in programs that require bidding. In some case the utility's or ISO's IT infrastructure will already support the notion of standing bids and in those cases it is not necessary for the DRAS to provide this functionality. In fact there may be scenarios where there are programs that require bidding, but all the bidding is handled by a 
different system than the DRAS, including the handling of standing bids. In this case, from the DRAS point of view, the program will not require bidding and all the DR events are simply issued by the utility or ISO as in the case of programs with no bidding. The utility or ISO will simply handle all the bidding as they normally would and use the DRAS to issue the DR events.

The remainder of this section assumes that the DRAS is handling the automated submission of standing bids. From the participant's point of view there is a so called bid state that they are in (see Figure 37). Normally the state is "IDLE" meaning that there are currently no outstanding requests for bids. This changes when the utility or ISO initiates a DR event for a program that may require bidding. In this case the DRAS will issue a request for bids by notifying the participant operators via email or some other means. The DRAS then tracks the bid state for each participant and can provide the current state information at any time for that participant. The state variables associated with each bid request include:

- Program-the program associated with requests for Bids.

- Notify time-the time that participants are notified of the request for Bids.

- Start time-the start time of the bidding

- End time-the end time of the bidding

- Bid info-this gives program and DR event related information related to bidding.

The Bid entity is used by both the utility or ISO and the participants to represent bids as described in Section 8.11. Bid state transition diagrams are shown in Figures 38 and 39.

There is a separate state diagram for each program and DR event that a participant may submit bids for. The bidding sequences in Figure 38 are initiated by the utility or ISO when they initiate a DR event for a program that requires bidding by the participant. In some cases the bidding is open and closed according to some fixed schedule which is not associated with a specific event. In this case the opening and closing of the entry of bids is simply according to some schedule. 


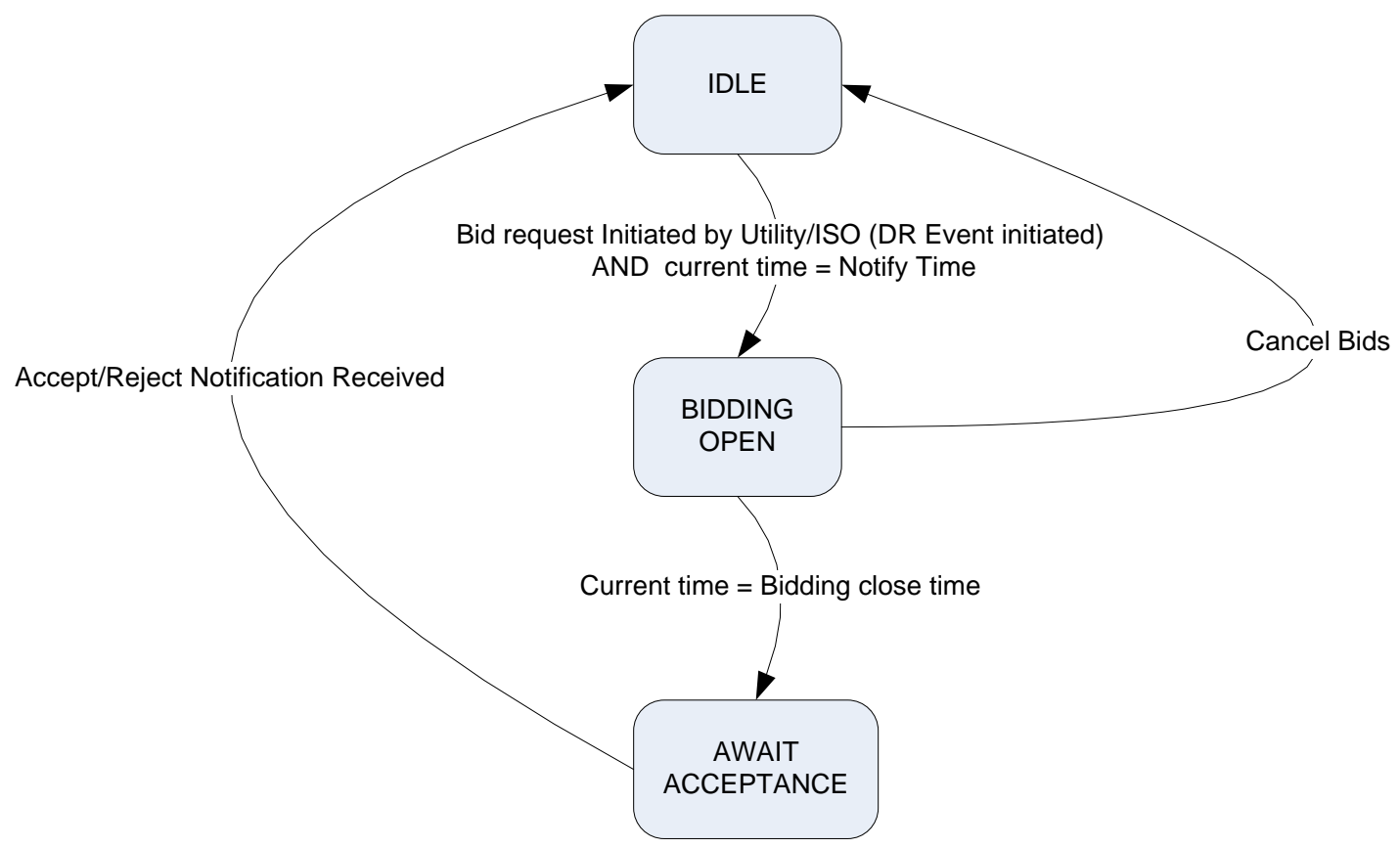

Figure 38. State Transition Diagram for a Participant's Bid State

Figure 39 shows the sequence diagram for the bidding process. Note the following:

- Step 3 is optional and if it is not performed then the standing bid for the participant is submitted.

- Step 4a represents the submission of the bid to the utility or ISO after the bid is submitted by the participant. If Step 3 is not performed by the participant then this step is not performed. This step may happen immediately after Step 3 or at a scheduled time (i.e. like Step $4 \mathrm{~b}$ in Figure 38).

- Step $4 \mathrm{~b}$ is performed at a specifically scheduled time and represents the submission of the standing bid if the participant does not manually submit a bid as part of Step 3. Since this happens at a scheduled time, participants have time to submit bids or change their standing bid.

Note that the DRAS should be configurable such that Step 4a is not performed immediately after it is submitted by the participant, but rather at a scheduled time like the standing bid is submitted.

- Steps 2 and 6 are machine to human communications and can utilize either email or some third-party notification system. 


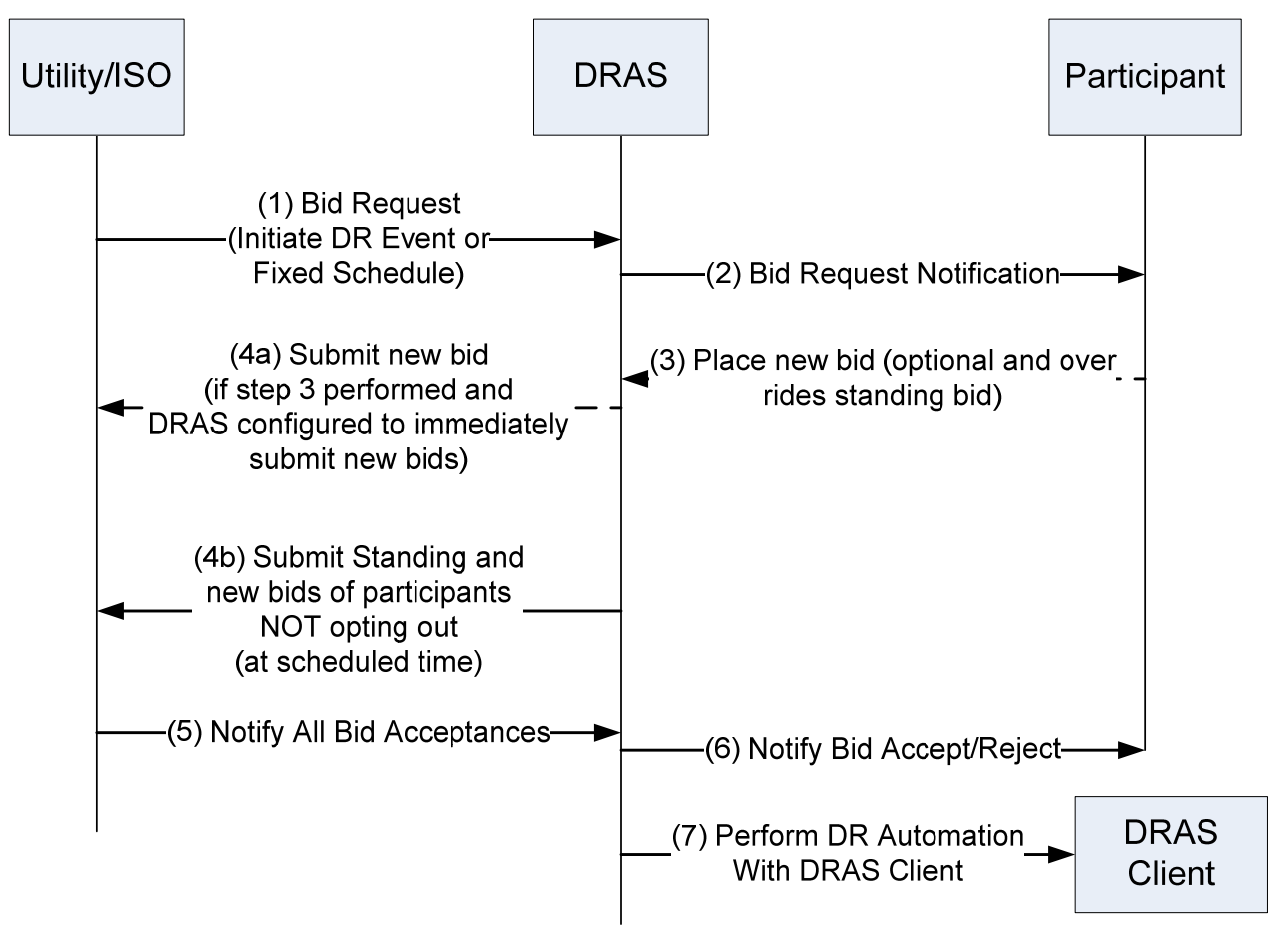

Figure 39. Bidding Sequence Diagram

When the utility or ISO notifies the DRAS of all bid acceptances (step 5), the acceptances come in the form of a list of UtilityDREvent entities that describe each of the DR events that need to be issued to the participants whose bids were accepted. This allows for the utility or ISO to customize a DR event for each participant that will reflect the bids that they made. Note that it is a requirement that each of the UtilityDREvent entities that are issued as part of the list of accepted participants have the SAME event identifier as the original DR event that was issued and initiated the request for bids.

Upon receiving the list of UtilityDREvent that signify the participants whose bids were accepted the DRAS will notify the participant operators (Step 6) and send the DR events to the relevant DRAS Clients (Step 7). 


\section{Functional Specifications}

This section gives a brief description of the various functions required by the DRAS in order to support the use cases and the interfaces described above for a DR event. These functions are organized according to which of the three interfaces described above that they belong to:

- Utility or ISO Operator Interface

- Participant Operator Interface

- DRAS Client Interface

For a more detailed description of each function in the various interfaces see Section 9.

\subsection{Utility or ISO Operator Functions}

These are the functions required by the utility or ISO if they are interfacing to a compliant DRAS. As noted earlier the DRAS functionality may be integrated with the utility's or ISO's IT infrastructure which means that these functions may not exist.

Note that except where noted in Section 9, users with the following security roles may access methods in this interface:

- All DRAS Operators

- All Utility and/or ISO Operators

\subsubsection{Utility or ISO Handling DR Events}

These are the functions associated with initiating and managing DR events for participants in a DR program and include the following functions:

- Initiate DR Event

- Edit or Cancel Existing DR Event

- Get Pending Event Information

Before a utility or ISO can use these methods to initiate a DR event the following configuration steps must have been performed by the utility or ISO:

- Participant accounts setup including defining DRAS Clients

- DR programs set up

Further configuration may be required by the participant before the DR event information can be received by a participant.

\section{InitiateDREvent}

This function is implemented on the DRAS and is used by the utility or ISO to initiate a DR event. The entire event information is passed in concerning the event along with a specification of the participants and or DRAS Clients that should receive the event. In 
the case where bidding is required as part of the program then this method call will initiate the bidding process.

\section{ModifyDREvent}

This function is used to edit a previously issued DR event. It must reference the DR event identifier that was assigned by the utility or ISO when the original event was issued. The following types of modifications can be made to an already initiated DR event:

- Cancel a DR Event

- Change the participant list

- Modify DR Event parameters

\section{AdjustDREventParticipants}

This function will modify the list of participants that will receive an already existing DR event. It is somewhat redundant with the functionality available with the more general ModifyDREvent method, but is deemed to be done frequently enough to merit its own method to make it easier.

\section{GetDREventInformation}

This function is used to get information related to currently pending or active DR events within the DRAS.

\section{$\underline{\text { SetEventConstraint }}$}

This method is used to set ProgramConstraint variables that are applied to a specific DR event.

\section{GetEventConstraint}

This method is used to fetch the ProgramConstraint variables that are applied to a specific DR event.

\subsubsection{Utility or ISO Support for Automated Bidding}

The following functions are used by the utility or ISO to manage the participant's bidding that is associated with a particular DR event. These functions include the following:

- Query the DRAS about existing bids

- DRAS send bids to the utility's or ISO's IT system

- Close the bidding

- Notify the DRAS of which bids were accepted or rejected The bidding process proceeds as described in Section 6.6. 
When participant bids are sent from the DRAS to the utility or ISO both a PUSH and PULL model is supported by the DRAS. In the PUSH model the bid information is sent by the DRAS to the utility or ISO by initiating the communications with the utility's or ISO's IT system. It does this by invoking a function on the utility's or ISO's IT system.

In the PULL model the utility or ISO queries the DRAS for the bidding information by invoking a function of the DRAS to retrieve the information.

\section{GetCurrentBids (PULL MODEL)}

This function allows the utility or ISO to request the current participant bids from the DRAS. The utility or ISO can either request bids for a specific event or all the standing bids for a specific program.

\section{SetCurrentBids (PUSH MODEL)}

This function is implemented on the utility's or ISO's IT system and allows the DRAS to proactively send the participant's bid information to the utility or ISO. It is beyond the scope of this document as to how this method's end point URL is specified and configured or how the DRAS' credentials for invoking this method are configured with the DRAS.

\section{CloseBidding}

This function is used to explicitly close the bidding for a particular DR event. When bidding is closed participants can no longer submit bids. When a DR event that requires bidding is issued it has a time in which the bidding will close. This function can close that bidding before that time.

\section{$\underline{\text { SetBidStatus }}$}

This function is used to notify the DRAS of which previously submitted participant bids have been rejected and accepted.

\subsubsection{Utility or ISO Configure DRAS}

These functions are used by the utility or ISO to configure the DRAS to support the DR programs and specifically the delivery of DR events to the participants in the DR program. The functions configure and edit the account and program information associated with a DR program. The various data model entities associated with DR programs from the utility's and ISO's point of view are described in Sections 6.4.1 and 8.

Depending upon the business process, the utility or ISO may also take responsibility for performing the configuration of the data entities normally configured by the participant. In that case the functions specified in Section 7.3 would also be performed by the utility or ISO. 


\subsubsection{Manage Programs}

The following functions are associated with managing DR programs within the DRAS. From the point of view of the DRAS a DR program is represented by the UtilityProgram entity and thus these functions represent the manipulation of that entity within the DRAS.

\section{CreateProgram}

This function is used to create a new DR program.

\section{ModifyProgram}

This function is used to modify an existing program that was created with the CreateProgram function.

\section{DeleteProgram}

This function is used to delete an existing program that was created with the CreateProgram function.

\section{GetPrograms}

This function is used to get all the information related to programs including the program constraints and event Info Types associated with the program.

\section{AdjustProgramParticipants}

This function is used to add or remove a participant from a program.

\subsubsection{Manage Participant Accounts}

The following functions are associated with managing participant accounts, and specifically the management of those accounts as they relate to programs. Each participant account is represented by a ParticipantAccount entity as described in Sections 6.3.4 and 8.5. A ParticipantAccount object is associated with an UtilityProgram object which allows the participant represented by that account to receive DR events from the DRAS. In addition a participant may have a set of ProgramConstraints that are specific to how that participant operates within a specific program. Note that these are different from the ProgramConstraints that were associated with the program as a whole and are specific to the participant. This is described in more detail in Section 6.5.2.

\section{CreateParticipantAccounts}

This function is used to create one or more participant accounts.

\section{ModifyParticipantAccounts}

This function is used modify existing participant accounts. 


\section{DeleteParticipantAccounts}

This function is used to delete participant accounts that were created with the CreateParticipantAccounts function.

\section{GetParticipantAccounts}

This function is used to fetch information related to participant accounts.

\section{GetGroups}

This function returns all the groups for all the participants.

\subsubsection{Utility or ISO Monitoring of DRAS Related Activities}

The DRAS logs various transaction and alarms as described in Section 6.3.2. The functions described in this section provide a means to query those logs.

\section{GetDRASClientCommsStatus}

This function is for retrieving a DRAS Client's current communication state.

\section{GetDRASTransactions}

This function is used to retrieve any of the transaction logs associated with the DRAS.

\section{GetDRASClientAlarms}

This function is used to retrieve DRAS Client Alarms that have been logged within the DRAS.

\section{GetParticipantFeedback}

This function is used to fetch a list of Feedback objects based upon a set of search criteria.

\subsection{DRAS Client Functions}

This section is a functional description of the methods required for the DRAS and DRAS Client to exchange information concerning DR events. As described above there is both a PUSH and a PULL model of interaction between the DRAS and the DRAS Client. In both cases an EventState entity is passed from the DRAS to the DRAS Client and a Confirmation message is sent from the DRAS Client to the DRAS to acknowledge receipt of the message. Section 8.12 gives a detailed description of the EventState and EventStateConfirmation entities.

This section only specifies the functional requirements of the methods that are to be implemented on the DRAS and the DRAS Client. Unlike other functions described in this section that are intended to be implemented using SOAP there are in fact a number of different ways in which the functions in this section can be implemented such as BACnet Web services and simple REST (Representational State Transfer). See Section 9.3 for a more detailed description of the actual methods that are used. 


\section{Send DR Event Information (PUSH) to DRAS Client}

This function is implemented on the DRAS Client. An EventState entity is sent by the DRAS to a DRAS Client whenever the participant's DR event state changes or when the DR event information has been changed by the utility or ISO. This includes canceling the DR event. When the DRAS Client receives the EventState entity it must send the DRAS an EventStateConfirmation entity as described in Section 6.5.3. Sending the confirmation message includes the DRAS Client invoking the method on the DRAS that is used for sending confirmation messages as described in Section 6.5.3. See Section 8.12 for a description of the EventState object.

\section{Get DR Event Information (PULL for DRAS Client)}

This function is used in the PULL model of interaction between the DRAS and DRAS Client to fetch information concerning any pending DR events. It logically operates the same as its PUSH cousin and allows the exchange of the same information. When the DRAS Client receives the EventState entity it must send the DRAS an EventStateConfirmation entity as described in Section 8.12. As part of the security policy the DRAS must ensure that the security credentials that were used by the DRAS Client to invoke this function match the various parameters passed into this function.

\section{Parameters}

- Participant ID

- Program ID

- DRAS Client ID (for example, username and password)

\section{Return Values}

- An EventState object as described in Section 8.12.

\section{Authorized Users}

- All DRAS Operators

- DRAS Client associated with the Participant ID

\section{Send Event State Confirmation to DRAS}

This function is implemented on the DRAS and is used by the DRAS Client to send confirmation that it has received a specific instance of an EventState object. If this function is not invoked by the DRAS Client within the time out period after the DRAS sends an EventState object the DRAS must assume that the EventState object was not received properly by the DRAS Client. As part of the security policy the DRAS must ensure that the security credentials that were used by the DRAS Client to invoke this function match the various parameters associated with the EventStateConfirmation object.

Note that the EventStateConfirmation object also contains a flag which specifies whether the DRAS Client is opting in or out of the DR event. An EventStateConfirmation object can therefore be used to initiate the opt-out state at the DRAS Client and to notify the DRAS of this state. Since the opt-out state can be set within the DRAS Client at any time, the 
EventStateConfirmation object can be sent at any time using a previously confirmed EventState.

\section{Parameters}

- EventStateConfirmation object as described in Section 8.12.

\section{Return Values}

- SUCCESS or FAILURE

\section{Authorized Users}

- All DRAS Operators

- DRAS Client associated with the EventState Confirmation object.

\subsection{Participant Operator Functions}

The functions described in this section are part of the participant operator interface. In general users with the following security roles may access the functions within this interface:

- All DRAS Operators

- All Utility or ISO Operators

- All Participant Managers

For security reasons if the role of the user accessing this method is a participant manager then the user name or password of the participant manager must match the user name or password on the participant accounts that are associated with the return values.

Also a few of the functions described in this section may be accessed by user with the DRAS Client installer security role.

\subsubsection{Opting Out of DR Events}

An opt-out state is a set of conditions for which the participant will not be participating in DR events. An opt-out state is represented by the OptOutState entity as described in Section 8.6, opt-out states can be based upon specific DR events, programs, or DRAS Clients. In addition there can be a schedule associated with an opt-out state. There may be multiple opt-out states associated with a participant and the DRAS must check each of the configured opt-out states to determine is a participant will receive a DR event. It is implementation specific for the DRAS to define what happens if an opt-out schedule starts or ends in the middle of a DR event period.

The functions in this section are for managing opt-out states for participants. Participant managers can only manage opt-out states for accounts that are associated with their ParticipantAccount.

The functions described in this section may also be used by the automation system in the facility in the use case where the opt-out state of the facility is set at the automation system and not through some specific user interface on the DRAS. Note that the opt-out 
state of a facility can also be set through the DRAS Client interaction by using the EventStateConfirmation which contains a flag that specifies whether the DRAS Client is opting out of a DR event.

\section{CreateOptOutState}

This function is used to create an opt-out state for a participant.

\section{DeleteOptOutState}

This function is used to delete a previously created opt-out state for a participant.

\section{GetOptOutState}

This function is used to fetch opt-out states for a participant.

\subsubsection{Submitting Feedback (Facility Status) to DRAS}

These functions are used by the participant to provide feedback concerning the status of the facility. The manner in which the feedback is provided might include a number of different actors including both human and machine which may include the DRAS Client.

\section{SetDREventFeedback}

This function is used to send information to the DRAS concerning the state of the facility and how the participant or DRAS Client reacted to the DR event being issued. Note that in general feedback may be sent at any time and may or may not be associated with a specific DR event.

\section{GetDREventFeedback}

This function is used to fetch a list of feedback objects that were created with the SetDREventFeedback function.

\subsubsection{Automated Bidding}

The functions described in this section are used by the participants to manage their bids that are associated with those DR events that require bidding. Bids are represented by the Bid entity. The bidding process is described in Section 6.6.

\section{SubmitStandingBid}

This function is used to set a participant's standing bid for a program.

\section{GetStandingBid}

This function is used to fetch a participant's standing bid for a program.

\section{DeleteStandingBid}

This function is used to delete a participant's standing bid for a program. 


\section{SubmitBid}

This function is used to set a participant's real-time bid for a program.

\section{GetBid}

This function is used to fetch a participant's real-time bid for a program.

\subsubsection{Configuration of Participant Related Information in DRAS}

These functions are used by the participants to configure the DRAS for the reception of DR events that are associated with a specific program. The following entities are managed by the participant and are involved in how they operate and receive DR events:

- ParticipantAccount

- DRASClient

- ResponseSchedule

- ProgramConstraints

Note that it is not a requirement that the utility or ISO allow access by any participant to the configuration functions detailed in this section. In that case all configuration functions would be performed by a utility or DRAS operator.

\subsubsection{Manage Participant Accounts}

The participant accounts are created by the DRAS or the utility or ISO operators. It is not required that the DRAS allow access to ParticipantAccount information by the participant. The DRAS may be configured to operate in a number of different ways:

- Participant has no access to ParticipantAccount information and the utility or ISO is responsible for all configuration of this information as described above.

- Participant may view, but can not modify any of the fields in the ParticipantAccount entity.

- Participant can both view and modify the information in the ParticipantAccount entity.

It is beyond the scope of this document to specify how the DRAS is configured to operate in one of the modes described above, but it is the assumption of this section that it is configured to operate in mode 2 or 3 as described above.

\section{GetParticipantAccounts}

This function is used to get the participant's account information (ParticipantAccount).

\section{ModifyParticipantAccount}

This function is used to modify existing participant accounts. While certain fields of the ParticipantAccount may be viewed by a participant manager, they may not be modified. These fields include the following: 
- Participant uid

- Participant name

- User name

- Program names

\subsubsection{Managing DRAS Clients}

Each participant may have multiple DRAS Clients associated with their account. These functions are used to manage the DRAS Clients.

\section{CreateDRASClient}

This function is used to create a DRAS Client. Note that program constraints and response schedules may be created through a separate set of functions and need not be created using this function.

\section{ModifyDRASClient}

This function is used to modify an existing DRAS Client.

\section{DeleteDRASClient}

This function is used to delete an existing DRAS Client.

\section{GetDRASClientInfo}

This function is used to fetch DRAS Client information associated with participant.

\subsubsection{Managing Program Constraints}

These functions are used to manage program constraints. A participant's program constraints are always associated with a particular program and in addition they are also associated with either the participant account as a whole or a specific DRAS Client.

Therefore these functions are used to manage both the program constraints that are for a participant as a whole as well as the constraints that are associated with a specific DRAS Client.

\section{GetParticipantProgramConstraints}

This function is used to fetch the ProgramConstraints object associated with the participant as a whole.

\section{SetParticipantProgramConstraints}

This function is used to assign the ProgramConstraints object associated with the participant as a whole.

\section{DeleteParticipantProgramConstraints}

This function is used to delete the Program Constraints object associated with the participant as a whole. 


\section{GetDRASClientProgramConstraints}

This function is used to fetch the ProgramConstraints object associated with a specific DRAS Client.

\section{SetDRASClientProgramConstraints}

This function is used to assign the Program Constraint object associated with a specific DRAS Client.

\section{DeleteDRASClientProgramConstraints}

This function is used to delete the Program Constraint object associated with a specific DRAS Client.

\subsubsection{Managing Simple DRAS Client Response Schedules}

As described in Section 6.5.3.3.2, response schedules are primarily intended for Simple DRAS Clients and are used to specify how to translate the EventInfo information associated with a DR event into the simpler Operation Mode variable transitions during the ACTIVE period of the DR event. The ResponseSchedule entity is used to define these translation rules and is associated with specific programs and DRAS Clients.

In order to create a ResponseSchedule for a DRAS Client the following information is required from the DRAS and may be used by a participant UI to create a ResponseSchedule:

- List of all EventInfoTypes that may be associated with the program in question.

- A consolidated set of Program Constraints that apply to a particular DRAS Client. If there is an explicit set of program constraints defined for a DRAS Client then those are used, else if there are program constraints defined for the participant as a whole then those are used, else the program constraints that are defined for the program as a whole is used.

Because of the way that program constraints are defined it is always possible to derive a set of ProgramConstraints that will be applied to a particular DRAS Client for a specific program.

With this information it should be possible to build a tool or editor that can be used to create response schedules.

The functions described in this section are used to manage the response schedules.

\section{GetProgramInformation}

This function is used to retrieve the information necessary to help an operator edit and specify a response schedule and its operating states. 


\section{CreateResponseSchedule}

This function is used to create a ResponseSchedule for a specific DRAS Client and program.

\section{DeleteResponseSchedule}

This function is used to delete a ResponseSchedule for a specific DRAS Client and program.

\section{GetResponseSchedule}

This function is used to fetch a ResponseSchedule for a specific DRAS Client and program.

\subsubsection{Monitoring of DRAS Related Activities}

The DRAS logs various transaction and alarms as described in Section 6.3.2. The functions described in this section provide a means to query those logs.

\section{GetDRASClientCommsStatus}

This function is for retrieving a DRAS Client's current communication state.

\section{GetDRASTransactions}

This function is used to retrieve any of the transaction logs associated with the DRAS.

\section{GetDRASClientAlarms}

This function is used to retrieve DRAS Client Alarms that have been logged within the DRAS.

\subsubsection{Installation and Testing of DRAS Clients}

These functions are used to test the communications between a DRAS Client and the DRAS. It does this by allowing the DRAS Client to be put into test mode. When a DRAS Client is in the test mode of operation then it is considered to be off line by the DRAS and does not receive normal EventState messages when DR events occur. If the DRAS Client is a Simple DRAS Client then these functions can be used to manually set the Operation Mode values of the DRAS Client. In the case of a Smart DRAS Client it can be used to test the reception of EventState messages.

\section{SetTestMode}

This function puts a DRAS Client into or out of test mode. If a DRAS Client is in test mode then it is essentially off line and will not receive any automated DR signals from the DRAS. 


\section{$\underline{\text { SetTestModeState }}$}

This function sends a test message to a DRAS Client and if it is a Simple DRAS Client then it can be used to set the operation mode and event state values. The DRAS Client must be in test mode for this method to work.

\section{GetTestModeState}

This function is used to get the current operation state of the DRAS Client if it is in test mode. 


\section{Detailed Data Models and Schemas}

The detailed schemas described in this section are all specified and documented using XML Schema Definition (XSD). All the documentation for the schemas is embedded in their respective XSD schema files. What is displayed in this section is simply a human readable form of that documentation that was extracted from the XSD file using a particular tool. If so desired the reader may take the machine readable source XSD file and browse it with a tool of their choosing.

The recent XSD versions are available on the LBNL Website- http://openadr.lbl.gov/src/. For example, http://openadr.lbl.gov/src/EventState.xsd refers to the EventState.xsd http://openadr source file.

\subsection{UtilityProgram}

See UtilityProgram.xsd

\subsection{UtilityDREvent}

See UtilityDREvent.xsd

\subsection{ResponseSchedule}

See $\underline{\text { ResponseSchedule.xsd }}$

\subsection{ProgramConstraint}

See ProgramConstraint.xsd

\subsection{ParticipantAccount}

See ParticipantAccount.xsd

\subsection{OptOutState}

See OptOutState.xsd

\subsection{Logs}

See Logs.xsd

\subsection{Feedback}

See FeedBack.xsd 


\subsection{Eventlnfo}

See EventInfo.xsd

\subsection{DRASClient}

See DRASClient.xsd

\subsection{Bid}

See Bid.xsd

\subsection{EventState}

See EventState.xsd 


\section{Detailed API Specifications}

The detailed Application Programming Interfaces (APIs) described in this section are for the most part specified using a Web Service Description Language (WSDL), which is the standard method for specifying a SOAP Web service interface. All the documentation for the methods and their parameters specified in the APIs are embedded in their respective WSDL files. What is displayed in this section is simply a human readable form of that documentation that was extracted from the WSDL file using a particular tool. If so desired the reader may take the source WSDL file and browse it with a tool of their choosing.

Recent API versions are posted on the LBNL Website (http://openadr.lbl.gov/src/) and include Bacnet DRAS Definition and DRAS Instance XML files For example, http://openadr.lbl.gov/src/bacnet.wsdl refers to the bacnet.wsdl source file.

\subsection{Utility Program Operator APIs}

This section details the methods in the API that are provided for the utility program operator methods as described in this section. There is a one to one correspondence between the functions described in that section and the method documentation presented here.

See UtilityOperator.wsdl

See UtilityInterface.wsdl

\subsection{Participant Operator APIs}

See ParticipantOperator.wsdl

\subsection{DRAS Client APIs}

This section details the methods of the API that are used for the communications between the DRAS and a DRAS Client. As previously discussed, the DRAS Client API is responsible for communicating DR EventState information to the DRAS Client in the participant's facility whether it is an end user or an aggregator. This API also includes the EventStateConfirmation message that is also sent from the DRAS Client to the DRAS upon receipt of the EventState information. See Sections 6.5.3 and 7.2 for a more detailed description of this interaction.

Many potential DRAS Clients are very limited in capabilities and not sophisticated enough to support a SOAP interface. Therefore there are two three distinct types of interfaces described here-one using a simple REST type of Web service interface, a simple SOAP type of Web service, and one using a more sophisticated SOAP interface that utilizes a BACnet Web Services (BWS) interface. 
The DRAS must support the BACnet simple SOAP Web service interface and may support may support the simple REST Web service interface and may support the BACnet SOAP Web service interface. Table 4 below indicates (with ' $X$ ") the required and optional services to be supported by this OpenADR specification and their supporting communication models.

Table 4Required Services and Supported Communication Models

\begin{tabular}{|l|c|c|c|}
\hline DRAS Client API & Required & PUSH & PULL \\
\hline Simple REST Services & & & $\mathrm{X}$ \\
\hline Simple SOAP Services & $\mathrm{X}$ & $\mathrm{X}$ & $\mathrm{X}$ \\
\hline BACnet SOAP Service (CSML) & & $\mathrm{X}$ & $\mathrm{X}$ \\
\hline
\end{tabular}

$\mathrm{CSML}=$ Control Systems Modeling Language

\subsubsection{Use of Simple REST Services to Exchange DR EventState Information}

It is recommended that DRAS Clients use a SOAP interface to the DRAS, but for DRAS Clients with limited communications capabilities that do not support a full SOAP protocol stack, the simple REST interface presented in this section is a means to interface to the DRAS and exchange DR event information. The REST interface must support all the requirements of the DRAS Security Policy as described in Section 10.

The REST interface does not support the PUSH model of interaction with the DRAS. A DRAS Client interacting with the DRAS uses Hyper Transfer Text Protocol (HTTP) Get to fetch EventState entities from the DRAS and subsequently uses HTTP Post to send the EventStateConfirmation messages back to the DRAS. This is depicted in the sequence diagram in Figure 40.

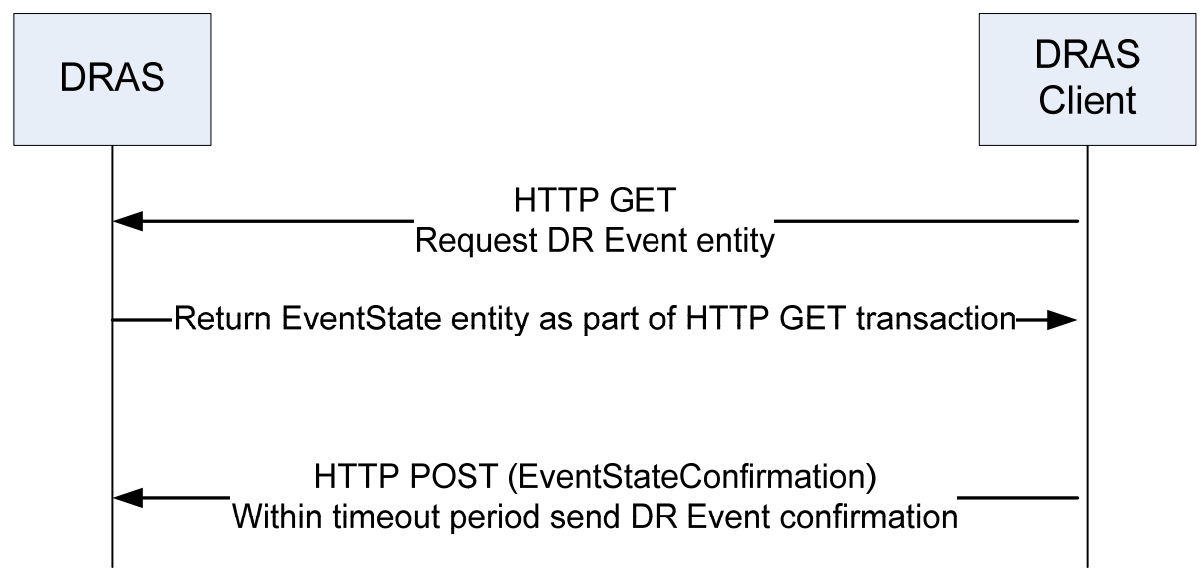

Figure 40. REST DRAS Client PULL Interaction Sequence Diagram

When the HTTP GET is invoked the DRAS returns an EventState entity. The EventState entity is documented in Section 8.12. 
When the HTTP POST is invoked to send the confirmation message an EventStateConfirmation entity is posted. The EventStateConfirmation entity is documented in Section 8.12.

Note that the path names used to access these services are implementation specific and not covered in this document.

\subsubsection{Use of Simple SOAP Services to Exchange DR EventState Information}

For DRAS Clients with capabilities to support a full SOAP protocol stack, the simple SOAP interface presented in this section is a means to interface to the DRAS and exchange DR event information. The simple SOAP interface must support all the requirements of the DRAS Security Policy as described in Section 10.

The DRAS and supporting DRAS Client should exchange EventState information using two different modes of interaction-PUSH and PULL-as described earlier in "Section 6.5.3.1, Modes of Interaction (PUSH versus PULL)." The following message exchanges must be supported by simple SOAP services:

- Using PULL architecture, the DRAS Client uses simple SOAP service to fetch the entire XML message of DR EventState information.

- Alternatively, using PUSH architecture, the DRAS uses simple SOAP service to send the entire XML message of DR EventState information to DRAS Client.

- The DRAS Client at the facility parses the values of EventState information.

- For both PUSH and PULL architecture, the DRAS Client at the facility acknowledges with EventStateConfirmation message to DRAS using simple SOAP service.

As shown in the sequence diagram Figure 41, in the PUSH mode of interaction the EventState information is "pushed" from the DRAS to the DRAS Client using simple SOAP service. This means, the communication of the EventState information is initiated by the DRAS. In terms of Web services this means that that DRAS Client is the Web server and the DRAS is the Web client. The DRAS Client must acknowledge the receipt of message using EventStateConfirmation message. 


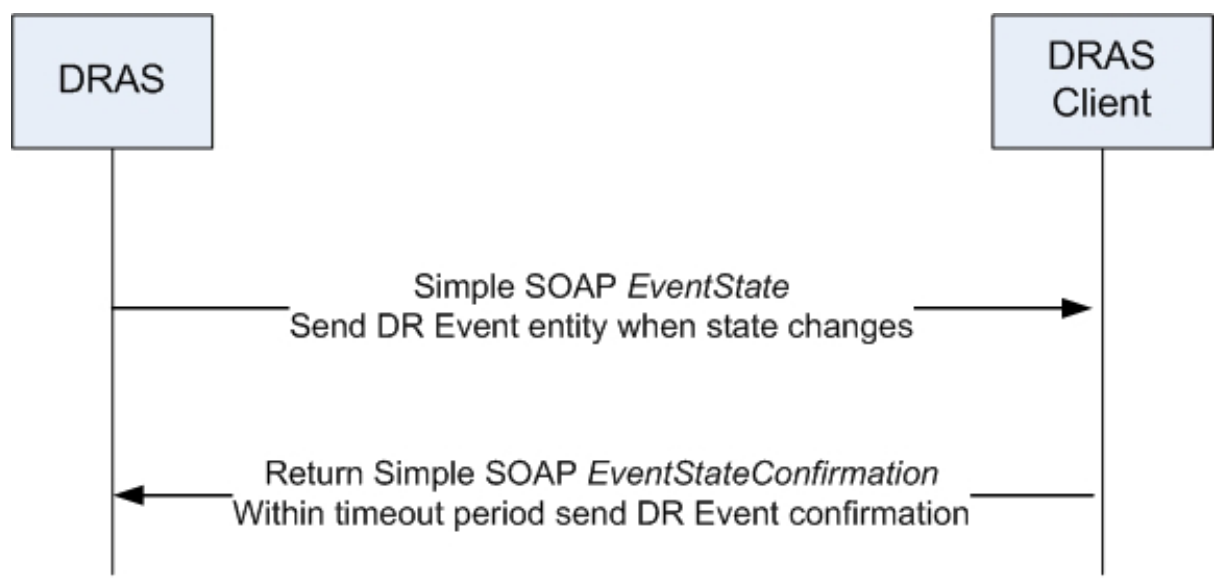

Figure 41. Simple SOAP PUSH Model Sequence Diagram

As shown in the sequence diagram Figure 42, in the PULL mode of interaction the EventState information is "pulled" from the DRAS by the DRAS Client using simple SOAP service. This means, the communication of the EventState information is initiated by the DRAS Client. In other words the DRAS Client polls the DRAS for the EventState information. In terms of Web services this means that the DRAS is the Web server and the DRAS Client is the Web client. The client must acknowledge the receipt of message using EventStateConfirmation message.

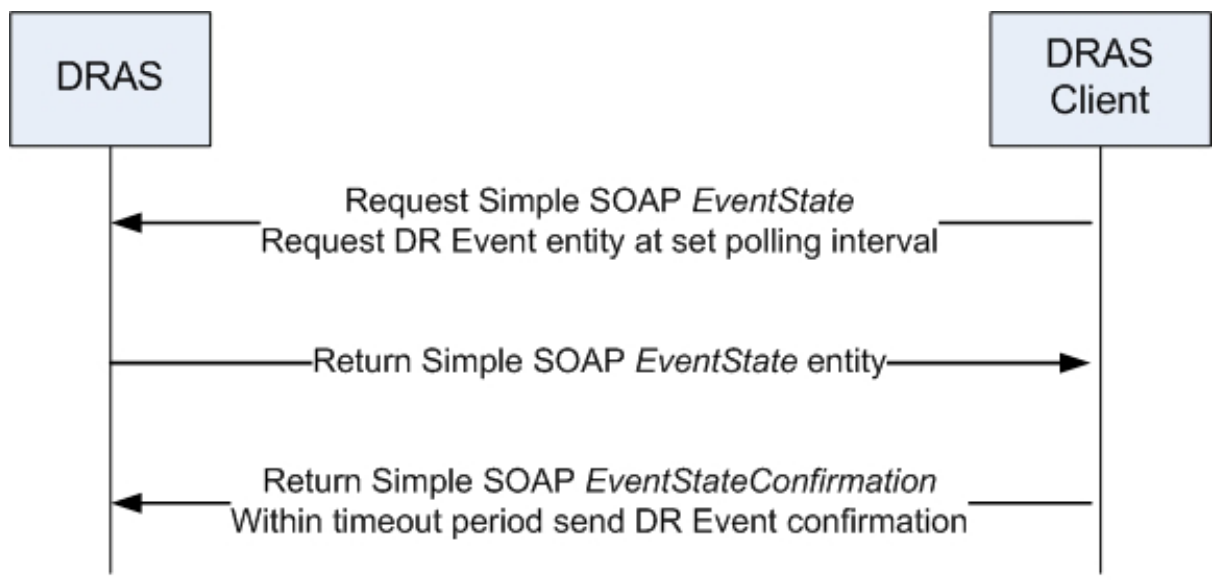

Figure 42. Simple SOAP PULL Model Sequence Diagram

\subsubsection{Simple SOAP Web Services API}

This simple SOAP services API and the minimum methods that must be supported are as described in Section 9.3.1 that are similar for simple REST services. The EventState entity is documented in Section 8.12 and the EventStateConfirmation entity is documented in Section 8.12. 


\subsubsection{Use of BACnet Web Services to Exchange DR EventState Information}

For those requiring interoperability with BACnet, this OpenADR specification may optionally use the BACnet Web Services (BWS) specification (ANSI/ASHRAE Addendum $C$ to Standard 135-2004) to communicate with BACnet-based systems.

The generic BWS data model allows interoperability with DRAS-issued DR event information and to schedule response strategies using Smart and Simple DRAS clients. The DRAS-BACnet Server and supporting DRAS Client should exchange EventState information using two different modes of interaction-PUSH and PULL-as described earlier in "Section 6.5.3.1, Modes of Interaction (PUSH versus PULL)."

To enable interoperability between the DRAS and BACnet-based controls implementing BWS, the following message exchanges must be supported:

- Using PULL architecture, the Client uses BWS' simple getValue service to read the entire XML message of DR EventState information as String value from the DRAS-BACnet Server.

- Alternatively, using PUSH architecture, the DRAS-BACnet Server uses BWS' simple setValue service to send the entire XML message of DR event state information as String value to the DRAS Client.

- The DRAS Client at the facility parses the String value of EventState information.

- For both PUSH and PULL architecture, the DRAS Client at the facility acknowledges with the String value of EventStateConfirmation information to the DRAS-BACnet Server using BWS' simple setValue service.

As shown in the sequence diagram Figure 43, in the PUSH mode of interaction the EventState information is "pushed" from the DRAS-BACnet Server to the DRAS Client to a pre-defined node path of a tree using setValue service. This means, the communication of the EventState information is initiated by the DRAS-BACnet Server. In terms of Web services this means that that DRAS Client is the Web server and the DRAS-BACnet Server is the Web client. The root of the tree is "DRAS" and the event state is written to node "DRAS/EventState." For real-time pricing this node is "DRAS/RTP." The DRAS Client must acknowledge the receipt of message using EventStateConfirmation message to the same node within specified timeout period using setValue service. 


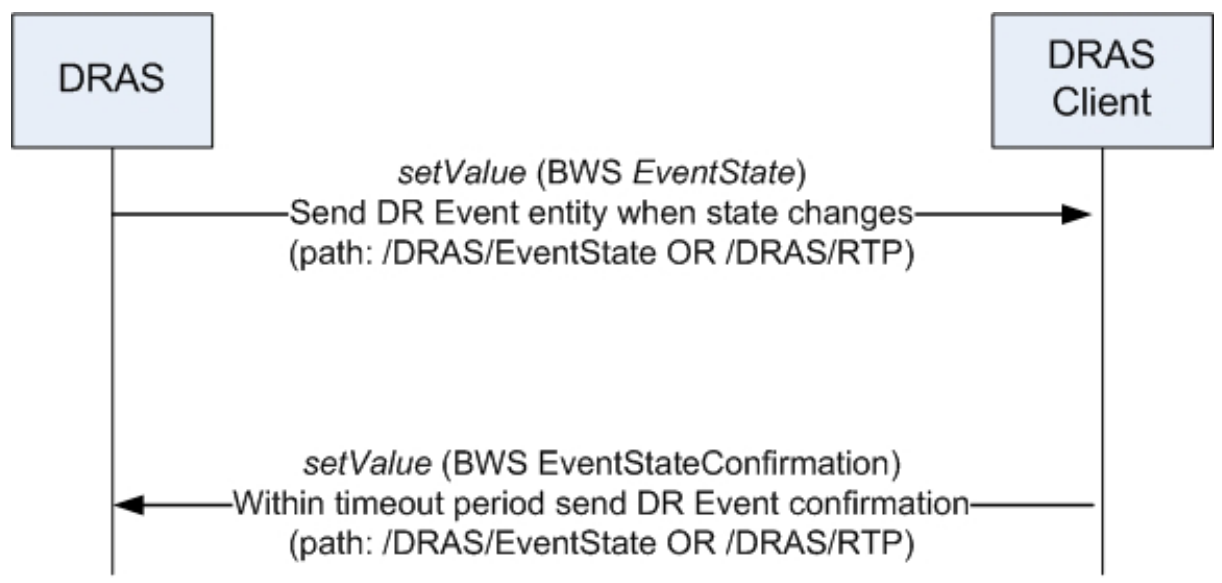

Figure 43. BWS PUSH Model Sequence Diagram

As shown in the sequence diagram Figure 44, in the PULL mode of interaction the EventState information is "pulled" from the DRAS-BACnet Server by the DRAS Client from a pre-defined node path of a tree using getValue service. This means, the communication of the EventState information is initiated by the DRAS Client. In other words the DRAS Client polls the DRAS-BACnet Server for the EventState information. In terms of Web services this means that the DRAS-BACnet Server is the Web server and the DRAS Client is the Web client. The root of the tree is "DRAS" and the event state is read from node "DRAS/EventState." For real-time pricing this node is "DRAS/RTP." The client must acknowledge the receipt of message to the same node using setValue service.

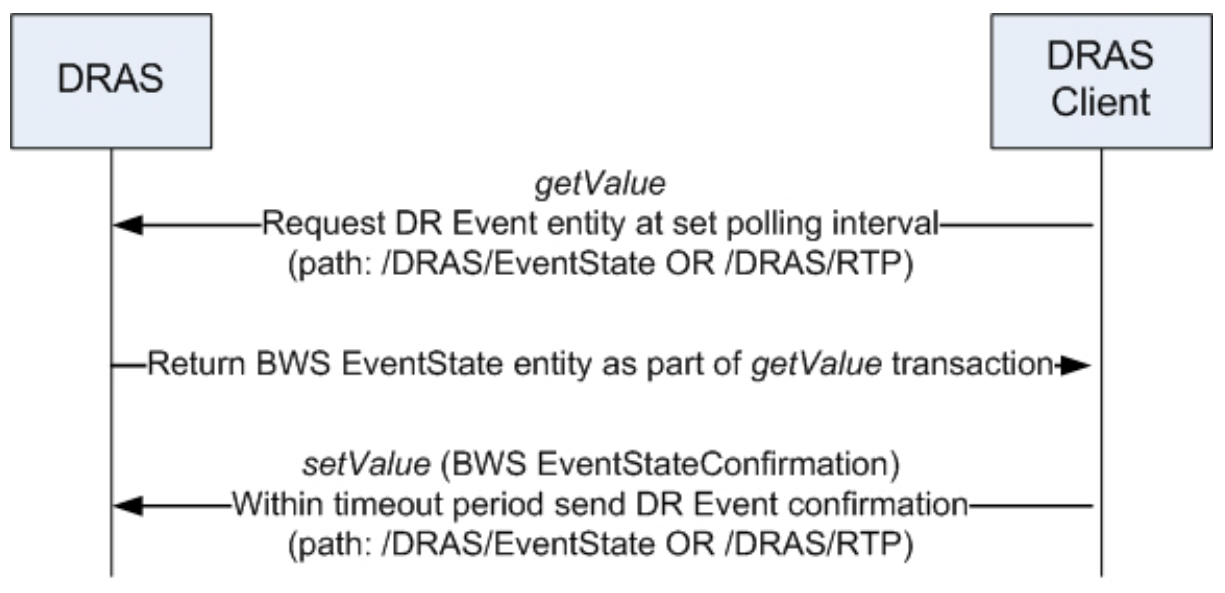

Figure 44. BWS PULL Model Sequence Diagram

\subsubsection{Services Supported by DRAS-BACnet Server}

While BWS has many services that support various aspects of control systems specifications, most of them are not relevant to the DRAS-BACnet server and getValue and setValue services are being used. The other two services getDefaultLocale and getSupportedLocals are required as per BWS specifications and must also be supported by 
the DRAS to exchange DR event data with the DRAS-BACnet server. These services are described in brief below. Refer BACnet Web Services (BWS) specification

(ANSI/ASHRAE Addendum $C$ to Standard 135-2004) for further details such as structure and procedures.

\section{getValue Service}

This BWS service is used to retrieve a single value for a single attribute of a single node. This service always returns its results as a single string.

This service can be used to retrieve primitive attributes, such as Value, and array attributes, such as PossibleValues. The format of this string result is dictated by the attribute's datatype and the service options.

If this service is used for an array attribute, then the array elements shall be concatenated into a single semicolon delimited string that can be easily split at the client since the element strings are not allowed to contain semicolon characters. If the client would rather retrieve an array of individual strings, it can use the getArray or getArrayRange service instead.

A typical programming language signature for this service is:

CString getValue(CString options, CString path)

\section{$\underline{\text { setValue Service }}$}

This BWS service is used to set a new value for a single attribute of a single node. The format of the new value is dictated by the attribute's datatype and the service options. This service always returns its results as a single string.

If the service option "readback" is true, then, after setting the value, this service shall read the value back and the result shall be as if the client had called getValue using the same path and service options. This allows the client to see the effects of any value modification by the server as well as check for errors.

Only the Value attribute is writable.

This service is required to be provided if the setValues service is provided.

A typical programming language signature for this service is:

CString setValue(CString options, CString path, CString Value)

\section{getDefaultLocale Service}

This BWS required service retrieves the locale that the server has configured for its default locale. The empty string ('"') shall be returned if there is no default locale, in which case the canonical form shall be used for all values. 
A typical programming language signature for this service is:

CString getDefaultLocale (CString options)

\section{getSupportedLocals Service}

This BWS required service can be used to retrieve the list of locales supported by the server. If the server does not support multiple locales, then this service shall return only the default locale. If the server does not support localization, and only uses the canonical form, then an array with no entries shall be returned unless the noEmptyArrays service option is true, in which case the result array shall contain a single entry for the WS_ERR_EMPTY_ARRAY error condition.

A typical programming language signature for this service is:

\section{CString[] getSupportedLocales (CString options)}

\subsubsection{EventState Schemas used for the BACnet Interface}

The DRAS-BACnet server EventState schema information is represented using Control System Modeling Language (CSML), which is both a data definition and data instance language. While the CSML file with DRAS information and data structure is machine and human readable and has an XML schema for validating the CSML file; however, the CSML in itself is a schema for control systems data model. The definition file defines the simple data structure of EventState schema and an instance file has instances of these data structures. Together these files represent implementation of devices, objects, and properties within a given device or collection of devices ${ }^{1}$.

XML schema is adequate for defining the structure of the CSML file, but is not rich enough to capture all the information that is needed to fully define a BACnet control system's data structure, presentation, and semantics. These definition and instance CSML files reference the schema defined in the supported BWS XSD file. An example of CSML implementation and supporting XSD are detailed below:

See DR-034E-22w2 Example DRAS Definition.xml

See DR-034F-22w2 Example DRAS Instance.xml

\subsubsection{BACnet Web Services API}

This section details the BWS services in the API that should be provided for the BACnet methods. This API includes all BACnet services and the minimum methods that must be supported are described in Section 9.4.2.

See bacnet.wsdl.

${ }^{1}$ http://xml.coverpages.org/facilitiesXML.html\#csml 


\section{Security Policy}

This section outlines the security policy of the communications with the DRAS and identifies the minimum security elements and interfaces that are required by this OpenADR specification.

\subsection{Scope}

In general there are many modes of attacks upon any sort of IT infrastructure ranging from intruders gaining physical access to the servers to remotely accessing the servers through open communication channels. This OpenADR specification only covers the communication protocols used to interact with the DRAS and the DRAS Clients. It is therefore only intended to cover modes of attack that would be perpetrated by using one of the communications channels that are used to implement the interface to the DRAS as described in the analysis section of Appendix C. Any other certainly necessary security measures (firewalls, intrusion detection, etc.) are not covered.

The DRAS has 3 distinct Web service interfaces, named after their principal users, who are subject to this security policy:

- Participant Interface

- DRAS Client Interface

- Utility Interface

The DRAS Client has one Web service interface (DRAS Interface) that is addressed by the DRAS.

As described above, both the DRAS and the DRAS Clients can act as a server or a client in a client/server relation.

\subsection{Access Control and Security Roles}

There are a number of types of users that require access to the DRAS. Each user may have different requirements on the type of functions they can perform and data they may access. To support limiting the access of the DRAS users based on their requirements, the DRAS must support the security roles outlined in Section 6.3.1.

These security roles are designed to limit access to the various methods in each of the Web service interfaces. Table 5 describes how each of the security roles is limited within each of the Interfaces. 
Table 5 Security Roles of Interfaces

\begin{tabular}{|l|l|l|l|l|}
\hline & \multicolumn{1}{|c|}{$\begin{array}{c}\text { Utility } \\
\text { Interface }\end{array}$} & \multicolumn{1}{|c|}{ Participant Interface } & $\begin{array}{l}\text { DRAS Client } \\
\text { Interface }\end{array}$ & $\begin{array}{l}\text { DRAS Interface } \\
\text { on DRAS Client }\end{array}$ \\
\hline DRAS & n/a* & n/a & n/a* & Full Access \\
\hline DRAS Operator & Full access & Full access & Full access & none \\
\hline $\begin{array}{l}\text { Participant } \\
\text { Operator }\end{array}$ & none & $\begin{array}{l}\text { Access to all methods, but } \\
\text { limited scope in what can } \\
\text { be done, viewed, etc. with } \\
\text { each method. }\end{array}$ & none & none \\
\hline $\begin{array}{l}\text { Utility Program } \\
\text { Operator }\end{array}$ & Full access & Full access & none & none \\
\hline DRAS Client & none & None & Full access & n/a* \\
\hline $\begin{array}{l}\text { DRAS Client } \\
\text { Installer }\end{array}$ & none & $\begin{array}{l}\text { Limited to a limited number } \\
\text { of methods used for testing. }\end{array}$ & none & none \\
\hline
\end{tabular}

${ }^{*}$ n/a-not applicable

For many of the functions described in this document the DRAS must limit the invocation of the data and methods that are accessible based upon the credentials of the user who is accessing the function. For example if a user with the participant operator role accesses the GetParticipantAccount function it must only have access to those ParticipantAccount entities that match the credentials of the user accessing the function. The various access restrictions based upon the security roles is documented with each function described above.

Additionally, for time-critical services, the DRAS can establish a connection to the DRAS Client's DRAS interface for performing a PUSH transaction.

All public communication interfaces are subject to the following requirements [RFC4949]:

- Confidentiality: the content of communication and the identity of users must be protected from third parties

- Integrity: communication must be protected from manipulation

- Authentication: communication is only allowed between authenticated and known partners

- Non-repudiation: transactions and message delivery can not be denied neither by the origin nor the recipient.

These requirements can be addressed via one of the following methods:

- DRAS Sec Method A: Secure tunnel with server-side certificates in conjunction with username or password client authentication

- DRAS Sec Method B: Secure tunnel with server-side and client side certificates

- DRAS Sec Method C: Web Services Security (reserved for future use, not described in this version) 
Additionally, authenticated clients have individual visibility of the respective server's data and services. This access control is specifically addressed in the detailed documentation for each method in the DRAS API. For each method the following is specified:

- The security role is allowed access to the method.

- For particular security roles the type of information is allowed to be returned for each method. For example a user with the participant manager role can only access information that is related to the participant account that they are associated with.

Security Management like key and certificate distribution, firmware updates, key revocation, etc. is implementation dependent and thus not in the scope of this document. The Secure Tunnel, Transport Layer Security Protocol (TLS), Version 1.0 (or newer) with Rivest, Shamir \& Adleman PK cryptography (RSA) extension has been chosen.

Certificate revocation lists must be used. Each transaction is a separate TLS Sessions that is terminated after the response. The following cipher suite choice reflects the most interoperable selection of state-of-the-art TLS APIs at creation of the OpenADR specification and shall be considered as a minimum level and must-have for the DRAS. The additional support for stronger ciphers is explicitly encouraged, as long as they are part of the official TLS specification as published by the Internet Engineering Task Force (IETF):

- Key exchange: RSA1024

- Data Encryption: 3DES (Data Encryption Standard), AES128 (Advanced Encryption Standard)

- Message Integrity Code (MIC): Secure Hash Algorithm (SHA1) - MIC: SHA1

- Message Authentication Code (MAC): Hashed MAC (HMAC) - MAC - HMACSHA1

The stable state of the system is that all API interfaces satisfy the above requirements up to a certain extent that corresponds to the particular threat level and system value. Reaching this state depends on the particular implementation. If the security of one interface depends on the security of another interface (e.g. a cryptographic key for interface A derived from a password that is received by a human user via interface $B$ and entered via interface $C$ ), it must be shown that the entire information chain satisfies the overall security requirements. Key distribution and management is therefore considered implementation dependent.

An implementation chooses the security measures for the non-API interfaces according to the usage scenario, threat levels, protected values, etc. The minimum level, given in this document, might (Client A) or might not (Client B and C) be right for a particular implementation as examples shown in Figure 45. Higher security measures can easily be integrated into the DRAS if necessary (Client C) as long as they are based on open 
standards. Communication partners with lower security levels (Client B) have to use a security proxy.

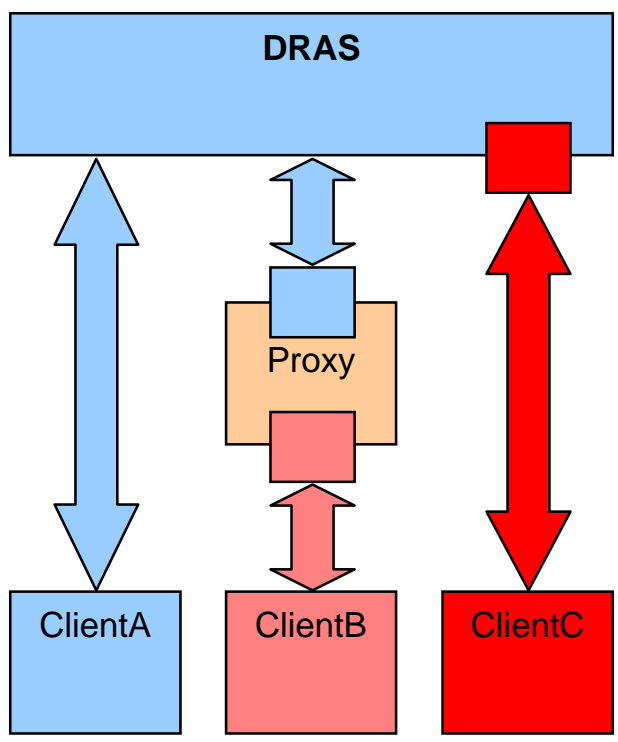

Figure 45. DRAS Communication Partners with Different Security Levels

The choice of security technologies like ciphers shall be limited to those that are open and freely available standards. 


\subsection{Future Developments}

This document represents a multi-year and multi-institutional effort to define how the DR signaling between utilities or ISOs and commercial and industrial facilities is performed to automate the process of issuing and responding to DR events and dynamic prices. Many aspects of the data models described in this document are already demonstrated and are in use in a variety of DR programs. The next steps in developing the OpenADR specification will include the following objectives:

- Expand OpenADR to additional DR programs and dynamic tariffs to gain more experience with new use cases.

- Engage a larger audience of stakeholders and industry experts to further develop the OpenADR specification.

- Continue collaboration with formal industry standards development organizations.

Objectives 2 and 3 are being satisfied by creating OpenADR groups within both the Utility Communications Architecture (UCA) International Users Group ${ }^{1}$ and Organization for the Advancement of Structured Information Standards (OASIS) ${ }^{2}$. Both these groups are highly respected with strong ties to formal standards groups like International Electrotechnical Commission (IEC) ${ }^{3}$. Future development of the OpenADR specification will take place within UCA and OASIS with the objective of moving OpenADR to a formal standards organization such as IEC. There will be a high degree of cooperation between UCA and OASIS and with each playing a complementary role in the further development of the OpenADR specification.

The official public review period was between May and July 2008, with comments accepted until November 2008. A significant number of comments were submitted by a variety of stakeholders. While many of the comments were addressed in this version, some will be addressed in future revisions within the UCA or OASIS. A detailed compilation of all the comments received can be found at http://openadr.lbl.gov/. The following is a general characterization of the type of issues that will be addressed in future OpenADR specification development:

- Feedback from Facilities to Utility or ISO: While the current version of the document has interfaces for providing feedback information from the facility to the utility or ISO, further analysis is required for more detailed specification(s) to address specific types of feedback such as control strategy or electricity load information.

- Security of Communications Channel: While a comprehensive security analysis is provided in this document, there are number of issues such as scalability, interoperability, and credential management that could benefit from further

\footnotetext{
${ }^{1}$ UCA International Users Group: http://www.ucaiug.org/

2 OASIS: http://www.oasis-open.org/

${ }^{3}$ IEC: http://www.iec.ch/
} 
analysis. In addition, it may be beneficial to harmonize the security policies with entities within OASIS and UCA.

- Network Scalability: Further detailed analysis for very high volume message traffic.

- Readability: Evaluate the need to categorize key elements of the specification. The current specification contains interfaces for the utility or ISO and participants. It may be beneficial to modify and separate the specification into more than one document, with the initial focus on the utility or ISO to participant communications.

- Expansion of Client Interfaces: Support additional DRAS Client interfaces such as Object Linking and Embedding (OLE) for Process Control (OPC) Unified Architecture (OPC-UA).

- Harmonization: Evaluate OpenADR harmonization with other industry efforts that are standardizing models for common data entities such as dynamic pricing.

- Ancillary Services: Explore the use of OpenADR in other markets such as wholesale and ancillary services.

- Implementation Guidelines: Develop OpenADR implementation and compliance guidelines to foster interoperability.

\section{Benefits to California}

OpenADR will provide benefits to California by both increasing the number of facilities that participate in demand response, and reducing the cost to conduct frequent and persistent participation in demand response. Furthermore OpenADR will improve the feasibility of achieving the state's policy goals of moving toward dynamic pricing, such as critical peak or real time pricing, for all customers. Increasing participation in demand response reduces the need for new electric supply, reduces the need for new transmission and distribution systems, and helps reduce overall electricity prices. 


\section{Definitions, Acronyms and Abbreviations}

For the purposes of this report, the following terms and definitions apply:

\begin{tabular}{|c|c|}
\hline AMI-SEC & Advanced Metering Infrastructure - SECurity \\
\hline API & Application Programming Interface \\
\hline Auto-DR & Automated Demand Response \\
\hline BACnet & Building Automation Control NETwork \\
\hline BIP & Base Interruptible Program \\
\hline $\mathrm{CBP}$ & Capacity Bidding Program \\
\hline CIP & Critical Infrastructure Protection \\
\hline $\mathrm{CPP}$ & Critical Peak Pricing \\
\hline CSML & Control Systems Modeling Language \\
\hline DBP & Demand Bidding Program \\
\hline DR & Demand Response \\
\hline DRAS & Demand Response Automation Server \\
\hline DRRC & Demand Response Research Center \\
\hline E-DBP & $\begin{array}{l}\text { Electronic Demand Bidding Program or (Automated) Demand } \\
\text { Bidding Program }\end{array}$ \\
\hline EMCS & Energy Management Control Systems \\
\hline GBP & Generic Bidding Programs \\
\hline GEBP & Generic Event-Based Programs \\
\hline HVAC & Heating, ventilation and air conditioning \\
\hline HTTP & Hyper Transfer Text Protocol \\
\hline IETF & Internet Engineering Task Force \\
\hline IP & Internet Protocol \\
\hline ISO & Independent System Operator \\
\hline IT & Information Technology \\
\hline $\mathrm{kW}$ & Kilowatt \\
\hline $\mathrm{kWh}$ & Kilowatt Hour \\
\hline NIST & National Institute of Standards and Technology \\
\hline NOC & Network Operating Center \\
\hline OASIS & $\begin{array}{l}\text { Organization for the Advancement of Structured Information } \\
\text { Standards }\end{array}$ \\
\hline PG\&E & Pacific Gas \& Electric \\
\hline PCT & Programmable Communicating Thermostat \\
\hline OpenADR & Open Automated Demand Response or Open Auto-DR \\
\hline PDC & Peak Day Credit \\
\hline REST & Representational State Transfer \\
\hline RFC & Request for Comment \\
\hline RTP & Real-Time Pricing \\
\hline SDG\&E & San Diego Gas \& Electric \\
\hline
\end{tabular}




\begin{tabular}{|l|l|}
\hline SHA & Secure Hash Algorithm \\
\hline SOAP & Simple Object Access Protocol \\
\hline TLS & Transport Layer Security \\
\hline UI & User Interface \\
\hline UIS & Utility Information System \\
\hline WSDL & Web Service Description Language \\
\hline XML & eXtensible Markup Language \\
\hline XSD & XML Schema Definition \\
\hline
\end{tabular}




\section{Appendix A: XSD Schema Files}

The following XSD schema files are posted at http://openadr.lbl.gov/src/, including both documentation and source code formats:

\begin{tabular}{|l|l|l|}
\hline$\underline{\text { Bid.xsd }}$ & 14-Jan-2009 12:08 & $3.4 \mathrm{~K}$ \\
\hline$\underline{\text { DR-034A-22w Schema.xsd }}$ & 15-Jan-2009 16:18 & $39 \mathrm{~K}$ \\
\hline$\underline{\text { DRAS.xsd }}$ & 14-Jan-2009 12:08 & $1.8 \mathrm{~K}$ \\
\hline$\underline{\text { DRASClient.xsd }}$ & 14-Jan-2009 12:08 & $11 \mathrm{~K}$ \\
\hline$\underline{\text { EventInfo.xsd }}$ & 14-Jan-2009 12:08 & $12 \mathrm{~K}$ \\
\hline$\underline{\text { EventState.xsd }}$ & 14-Jan-2009 12:08 & $25 \mathrm{~K}$ \\
\hline$\underline{\text { FeedBack.xsd }}$ & 14-Jan-2009 12:08 & $4.8 \mathrm{~K}$ \\
\hline$\underline{\text { Logs.xsd }}$ & 14-Jan-2009 12:08 & $5.7 \mathrm{~K}$ \\
\hline$\underline{\text { OptOutState.xsd }}$ & 14-Jan-2009 12:08 & $4.5 \mathrm{~K}$ \\
\hline$\underline{\text { ParticipantAccount.xsd }}$ & 14-Jan-2009 12:08 & $11 \mathrm{~K}$ \\
\hline$\underline{\text { ProgramConstraint.xsd }}$ & 14-Jan-2009 12:08 & $13 \mathrm{~K}$ \\
\hline$\underline{\text { ResponseSchedule.xsd }}$ & 14-Jan-2009 12:08 & $9.8 \mathrm{~K}$ \\
\hline$\underline{\text { UtilityDREvent.xsd }}$ & 14-Jan-2009 12:08 & $9.7 \mathrm{~K}$ \\
\hline$\underline{\text { UtilityProgram.xsd }}$ & 14-Jan-2009 12:08 & $9.8 \mathrm{~K}$ \\
\hline
\end{tabular}


APA-2 


\section{Appendix B: WSDL Interface Files}

The most recent versions of the following WSDL interface files and API specifications, including documentation and source code formats, are posted at http://openadr.lbl.gov/src/):

\begin{tabular}{|l|l|l|}
\hline DRASClientBACnet.wsdl & 14-Jan-2009 12:08 & $4.0 \mathrm{~K}$ \\
\hline ParticipantOperator.wsdl & $14-J a n-200912: 08$ & $110 \mathrm{~K}$ \\
\hline$\underline{\text { UtilityInterface.wsdl }}$ & $14-J a n-200912: 08$ & $2.5 \mathrm{~K}$ \\
\hline$\underline{\text { UtilityOperator.wsdl }}$ & $14-J a n-200912: 08$ & $66 \mathrm{~K}$ \\
\hline$\underline{\text { bacnet.wsdl }}$ & $14-J a n-200912: 08$ & $12 \mathrm{~K}$ \\
\hline
\end{tabular}


APB-1 


\section{Appendix C: Security Analysis and Requirements}

This appendix provides a high-level security analysis of the various risk factors associated with the Open Automated Demand Response Communications Specification, also known as OpenADR or Open Auto-DR. The appendix also presents a set of security requirements based on this analysis.

\section{C.1 Assumptions}

The following assumptions form the basis of the OpenADR security analysis:

This document is an Application Programming Interface (API) specification. Therefore for the sake of this discussion, the security perimeter of the DRAS encompasses the functions that define the API. The only malicious activities considered within the scope of this document are those that may be enabled by someone using one of the functions defined by this document. The malicious activity itself need not be the actual use of one of the API functions. For example, the case where third parties may be monitoring the activity of an authorized user's access of an API function which subsequently enables some other type of malicious activity that may not include the API function (i.e., stealing sensitive information).

All the functions defined in this document are implemented using industry standard Web services. This means that there is a well defined data model (typically utilizing HTTP) that defines how the functions are invoked. Web services typically utilize IP networks and more specifically the Internet, although this may not be necessarily

All users have a set of credentials and must identify themselves to the DRAS to use any of the functions defined in this document. Note that certificate management and distribution are for the actual server and client implementation and not part of the OpenADR data model itself. Also, depending on communications technology selection, user certificates will play a role in securing the client/server data communications architecture. The details of certificate management for this purpose are outside the scope of this document.

This specification assumes that there are well defined security roles for users that restrict the scope of the user's access (machine to machine and human to machine) to functions defined in OpenADR and the data that can be accessed through those functions. The management of these roles as well as their scope and granularity, while important in understanding OpenADR operations, are outside the scope of this document.

\section{C.2 Existing Security Standards}

It is the intent that the OpenADR specification leverage existing security policies and standards when applicable. The following policies and standards are therefore relevant to this effort:

- North America Electric Reliability Corporation (NERC) Critical Infrastructure Protection (CIP) CIP-002 through CIP-009 
- National Institute of Standards and Technology (NIST) SP800-82 Guide to Industrial Control Systems (ICS) Security

- Organization for the Advancement of Structured Information Standards (OASIS)Web Services Security Standards

- Open Advanced Meter Infrastructure Security (OpenAMI-SEC) Policies

It should be noted that AMI-SEC (Advanced Metering Infrastructure - SECurity) has performed an in-depth analysis of a wide range of risk factors and objectives that are within a domain similar to that of the DRAS. In many respects the scope of the AMI-SEC analysis is much broader than what is addressed by the DRAS. Where there are relevant use cases and analyses, the security policy of the DRAS should satisfy the analysis and requirements as established by the AMI-SEC working group.

It should also be noted that because the DRAS is utilizing Web services as the infrastructure to implement the specified functions, the DRAS should adhere to the security principles and policies as specified by OASIS and other committees or organizations, such as the Internet Engineering Task Force (IETF), Web Services Interoperability (WSI), World Wide Web Consortium (W3C), etc., that specialize in specifications for transactions and interactions that utilize the Internet-in particular, those involved in electronic commerce transactions.

\section{C.3 DRAS Risk Context}

This document primarily describes a set of APIs and the communications services required to support the semantics of those APIs. It also contains a suitable information model that is capable of supporting both client and server roles of a distributed demand response client/server system architecture. When taken as a whole, they provide a reasonably complete set of communication and semantic requirements for the functional aspects of a modern DRAS system. Having said this, no specification of a modern, distributed computing application can be considered complete without a thorough discussion of both the implicit and explicit security requirements needed to ensure its correct behavior.

Security issues intersect with OpenADR implementations on two fundamental levelseach of which is associated with a different set of risks. For the purpose of analysis, assume that communications between participating DR systems is reliable, private and authenticated. This means the following:

- The message byte streams sent by either client or server arrive at their destination correctly-the received byte stream is a full fidelity copy of the sent stream.

- The messages sent in either direction are not viewed or interpreted by any third party-they are considered private.

- The receiver of each message can be absolutely certain about the identity of the message sends-i.e., that the true identity of the sender is known. 
Given this ideal and, unfortunately, unrealistic depiction, operation of an OpenADR specification is reasonably secure-but not completely without risk. The facility manager who manages a DRAS Client could decide to not honor their commitments to shed loads when requested. The facility manager could refuse to honor their bid obligations or, by utilizing an unforeseen feature of energy pricing policy, attempt to "game" the system. And, of course, a latent design or coding error in a particular OpenADR implementation could surface and paralyze the entire system for an extended period of time. All of these activities would, in some way, put the OpenADR operation and the utility or ISO enterprise at risk. And, like the unforeseen "hacker" attack (see details below), they can happen with little warning and have substantial operational and monetary effects. However, as risks, these activities are reasonably foreseeable and, to a large degree, can be mitigated through good application, business practices, and policies within the OpenADR enterprise itself. Similarly, in the software implementation, diligent application of accepted, best-practice software engineering methods can substantially reduce the risks associated with technical failures in implementing the OpenADR data model (e.g. misconfigured systems, lost or corrupted transaction log files, or poorly implemented system privilege management and password access control).

Unfortunately, for automated DR purposes, OpenADR is of little value unless it is deployed in a widely-distributed environment-an environment with a multitude of risks for reliable data communications. Like all widely-distributed systems, the exposure of critical program data communications functions to real-world conditions creates increased operational risk of malicious attacks. Real world deployment of OpenADR, therefore, requires additional efforts to secure reliable and robust system operation. Specifically, these efforts are centered on securing client server communications. However, it is worth differentiating the types of new risk exposure encountered when deploying OpenADR in the "wild". Since DRAS Clients and servers are, in fact, computer systems, they are subject to attempts by hackers to log in and obtain sufficient system privileges to carry out some form of malicious attack. While this would clearly interrupt or curtail OpenADR operations, the root cause, unauthorized access to the system, is a problem for all computers directly connected to the Internet. There is little that the OpenADR specification can do to strengthen system security against illegitimate logins than, perhaps, require that it be executed on systems that enforce industry "best practices" to prevent unauthorized access. However, since the OpenADR architecture is based on reliable, private, authenticated communications between system components, it is important that this specification require additional measures to secure data communications. For this reason, great effort has been taken to specify "best practices" security implementations, such as Web services security and Transport Layer Security (TLS), for data communications.

It is worth noting that implementations of these OpenADR specifications will never, in a meaningful way, exist outside of a larger system context that are part of the infrastructure. This larger context, which includes features such as utility or ISO to DRAS connectivity, operational policies for system log security, mechanisms for 
assigning roles to individual users, key distribution, etc., contains a number of system elements and process outside the scope of this document. And, although these additional system elements interact closely with the core functions described here, they bring along additional unique risks. While the core functions described in this OpenADR specification are only meaningful when implemented as part of a larger, operational DR system, there are critical areas of the infrastructure, taken as a whole, that are correctly beyond the scope of this document. In other words, even if the core functions described in this document are implemented in a perfectly secure fashion, the overall robustness of the OpenADR enterprise is dependent on risks found at both the OpenADR specification, DRAS, and associated systems.

\section{C.4 DRAS Sources of Risk}

Table C1 lists a general classification of the types of activities that may adversely affect DRAS operations. The severity of risk is on a spectrum of 1 to 3 with 1 indicating the most severe.

Table C1. DRAS Sources of Risks and General Classification

\begin{tabular}{|l|c|}
\hline Accidental or non-Malicious Activities & Severity \\
\hline $\begin{array}{l}\text { Operational errors by either utility or ISO and customer that limit a customer's } \\
\text { ability to respond to DR events. This could range from utility or ISO database } \\
\text { data entry errors to incorrect equipment control settings at customer sites. }\end{array}$ & 3 \\
\hline $\begin{array}{l}\text { Minor telecommunications equipment failures that affect only a portion of } \\
\text { DRAS Clients. }\end{array}$ & 3 to 2 \\
\hline $\begin{array}{l}\text { Major IT equipment failure at A DRAS site or widespread, regional } \\
\text { telecommunications outage. }\end{array}$ & 1 \\
\hline $\begin{array}{l}\text { Fatal DRAS software implementation flaws that surface under unusual or } \\
\text { unexpected circumstances. }\end{array}$ & UNKNOWN \\
\hline
\end{tabular}

\begin{tabular}{|l|c|}
\hline Malicious, non-Communications Related Activities & Severity \\
\hline Participant denies submitting bid & 2 \\
\hline Participant denies receiving DR event information & 2 \\
\hline Intentional manipulation of DR events issued by the DRAS. & 3 \\
\hline $\begin{array}{l}\text { Manipulation (i.e. "gaming") of the DRAS system by one or more customers } \\
\text { on discovery of unintentional flaw in system software implementation. }\end{array}$ & 3 \\
\hline
\end{tabular}

\begin{tabular}{|l|c|}
\hline Malicious Communications-based Activities & Severity \\
\hline $\begin{array}{l}\text { Flood the DRAS communications channel with non-DR related Internet traffic } \\
\text { (Denial of Service attack). }\end{array}$ & 1 \\
\hline $\begin{array}{l}\text { Modify existing configuration data in the DRAS including programs, } \\
\text { participants, etc. }\end{array}$ & 1 \\
\hline
\end{tabular}




\begin{tabular}{|l|c|}
\hline Issue spurious or detrimental DR events & 1 \\
\hline $\begin{array}{l}\text { Modify existing DR events, including canceling them and changing which } \\
\text { DRAS Clients receive the events. }\end{array}$ & 1 \\
\hline $\begin{array}{l}\text { View data within the DRAS including participant information and user } \\
\text { activities. }\end{array}$ & 1 \\
\hline View private data contained in messages flowing to and from the DRAS. & 1 \\
\hline $\begin{array}{l}\text { Potentially gain access to a DRAS Client's credentials and directly access the } \\
\text { DRAS Client (e.g. "man in the middle attack"). }\end{array}$ & 1 \\
\hline Disable the DRAS Client from receiving DR events & 1 \\
\hline Manually issue messages to DRAS Client & 1 \\
\hline Shut down all DRAS operations & 1 \\
\hline Shut out access to other operators & 1 \\
\hline Submit Bids for participants & 1 \\
\hline Reject/Accept Bids & 1 \\
\hline Mimic the DRAS Client and intercept DR event messages & 1 \\
\hline Submit false feedback information & 1 \\
\hline Participant denies submitting bid & 2 \\
\hline Participant denies receiving DR event information & 2 \\
\hline
\end{tabular}

\section{C.5 Adverse Risk-Related Effects}

One important observation is that, regardless of the malicious or accidental nature of the above risks, when they are encountered, their effect is to limit the ability of customers to respond to DR signals. Some failure scenarios will cause longer disruptions than others and the geographical extent of the disruption will vary with the nature of the action. But the results are substantially the same. For example, some, or all, of the DR enterprise could be inoperable for some length of time. As a result, these risks have real and tangible, costs associated with them. Every hour that some, or all, of the DR enterprise is "off line", both Utilities and customers are denied the opportunity to optimize the cost of respective operations. But, there does not appear to be a clear signature that differentiates the kinds of adverse results that follow from an accidental error or a truly malicious attack.

It should also be noted that these actions, either accidental or malicious, should not present any increased risk to either the safety of utility or ISO and customer operations staff or to major electrical equipment. Given the scope of DR activities, as defined in this specification, DR events are not intended to be part of time critical, closed loop control designs that could adversely affect the safety of either personnel or equipment. While Auto-DR systems are clearly intended to be reliable and robust over long periods of time, by their distributed nature, they are always at some risk of suffering a communications failure-malicious or otherwise. The inclusion of OpenADR mediated 
load control as an essential, system-critical part of a closed loop dispatch design would be inappropriate. Therefore, at the highest level, the OpenADR applications should be designed to be "intrinsically safe" in the presence of unexpected communications failures-accidental or malevolent.

While it is clear that OpenADR systems should be designed to avoid any potential harm to people or equipment, systems can also be designed, using the existing specification, to detect and ignore "unreasonable" DR events that could otherwise trigger large, automated reductions in power use. Consider a DRAS Client that receives a DR event indicating a large and unsupportable increase in the cost of electrical power. This event could be the result of the utility or ISO operator error, malicious "hacker" activity following a communications security breech, or even a yet-undiscovered programming "bug". Regardless of the source of this "unreasonable" DR pricing event, DRAS Clients could, if not properly protected, automatically respond by shedding substantial power loads in order to avoid drastically increased power costs. While this operation may be safe, in the above sense of not endangering people or equipment, it may prove costly to large-scale industrial processes or to commercial building occupants during periods of extreme cold weather. Fortunately, OpenADR has a mechanism for detecting such unusual DR events and placing all potentially automatic changes for these events in a "pending" state, subject to DRAS Client approval. This verification can be as simple as calling the local utility or ISO dispatch office or receiving a confirming email from the relevant DRAS. The critical point is that, regardless of their accidental or malicious nature, the OpenADR specification contains mechanisms for trapping critical or unusual DR events and requiring independent verification before taking any action.

Ultimately, the result of persistent or chaotic communications malfunctions (accidental or malevolent) is the potential erosion of trust or reputation between an OpenADR enterprise and its customers. This is, perhaps, the most insidious and least understood major risk. The motivation to participate in a DR program is derived from a customer's ability to exchange reduced flexibility in electrical power consumption for a monetary value. OpenADR technology provides the mechanism to accomplish this goal by managing the exchange while minimizing the effort required on the part of the consumer. Although operational problems arise in almost any enterprise, the assumption borne out by daily experience is that, eventually, these problems can be resolved if the appropriate parties communicate. However, if unknown third parties intrude into the business relationship and instigate chaotic or, even worse, malevolent activity, then the element of trust will quickly erode. One need only look at the level of effort that successful Internet vendors put into their customer support centers to understand how critical trust is to computer communications mediated relationships.

As noted earlier, when OpenADR systems become inoperable or partially ineffective, both Utilities and customers incur both real losses and missed "opportunity costs." Since the results of either accidental or malicious actions are essentially the same, risk reduction in all the areas noted above will, in aggregate, improve the cost effectiveness of automating DR. It is clear that any reduction in the risk exposure for data 
communications disruption will benefit the OpenADR enterprise. In large part, this is exactly the risk profile experienced by most electronic commerce-based businesses. They are at risk from both internal and external actions, accidental and malicious, that ultimately drive their financial success. Therefore, the security model they apply to data communications reliability is, in large part, directly applicable to the needs of the OpenADR enterprise. By leveraging the risk analysis seen in the electronic commerce domain, one can take advantage of the larger community of interests that are focused on securing distributed data communications and, ultimately, share much of the common security infrastructure that has been developed. The primary security area focused on within this OpenADR specification, namely secure distributed data communications, are the security standards in general use by electronic commerce applications, TLS and Web services, are appropriate for addressing OpenADR communications security risks.

\section{C.6 Security Requirements}

The following set of general security requirements is derived from the risk analysis above:

- All of the Web service methods defined by the various DRAS interfaces should be accessible only by authorized users.

- The DRAS must prevent the access of sensitive information stored within the DRAS except by authorized users. Sensitive information is defined as any information that would not otherwise be publicly available.

- Information stored within the DRAS must not be modified except by authorized users.

- The DRAS must protect the confidentiality of the participants, utilities and ISOs that are using the DRAS. This means that the identity of the parties accessing and using the DRAS should be kept confidential from all unintended users.

- Information exchanged with the DRAS must maintain confidentiality and integrity from $3^{\text {rd }}$ parties (protection from inspection and interference from unintended users) while it is in transit to or from the DRAS.

- It must be possible for interfaces on utility or ISO and participant sites that are accessed by the DRAS (i.e. PUSH mode of operation) to be made secure such that those interfaces can only be accessed with the proper credentials.

- The DRAS must provide alternate channels of notification in order allow humans to verify and authorize interactions with the DRAS in scenarios where it is operationally required to have multiple levels of verification and authorization before any actions be taken in response to a message from the DRAS. An example of such an alternate channel might be an email or voice message to an operator.

- The DRAS must support the non-repudiation of the following transactions:

a. Bids submitted by participants 
b. Reception by participants of DR event messages

- Where applicable the DRAS should adopt security methods and policies from other standards bodies such as NIST, OASIS or UtilityAMI (Utility Advanced Metering Infrastructure). 


\section{Appendix D: DR Program Use Cases}

This section lists use cases related to the Open Automated Demand Response Communications Specification, also known as OpenADR or Open Auto-DR. Most use cases are derived from actual DR programs and dynamic tariffs in operation today. Some are either generalizations of exiting programs, or envisioned as yet to be defined DR programs. It is not intended to be an exhaustive list of all known DR programs and potential dynamic tariffs, but a representative sampling.

The descriptions of the DR programs and dynamic tariffs listed in this section rely on specific programs currently being offered, but should be viewed as examples that are used within the context of an automated DR program. The use cases should not be taken to literally reflect how the current DR programs operate, but how they might operate in an automated fashion with a DRAS.

\section{D.1 General Use Case Definitions and Nomenclature}

Each use case consists of the following documentation:

- A textual description of the elements of the use case.

- A diagram that depicts the relationship between various roles, actions, and systems in the use case.

- A set of use case scenarios with accompanying procedures and steps that describe the sequence of actions that are part of that scenario.

\section{D.1.1 Use Case Elements}

Each use case's elements or components include systems, actions and roles.

\section{D.1.1.1 Systems}

In use case diagrams, systems are denoted by rectangles that encompass actions, roles and other sub-systems (See Figure D1).

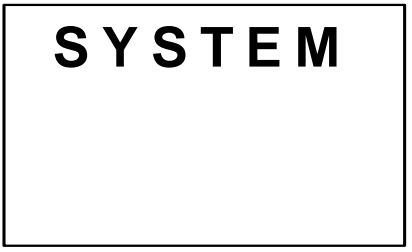

Figure D1. Use Case System

The use cases that follow consist of the following systems:

- Utility: This is the entire utility or ISO organization that is responsible for the distribution of electricity.

- Utility Information System (UIS): This is the IT system and software within the utility that is used to manage the Utilities operations. Much of what is depicted 
in this sub-system in the use case are not directly related to the DRAS, but included anyway for completeness in depicting the DR programs. Thus even though what is depicted in the use case may vary considerably between different Utilities, it is the goal of this effort that such differences do not effect the operation of the DRAS.

- DRAS: This system is the focus of this document.

- Facility: The building or factory where electricity is consumed.

- Aggregator: A third party responsible for managing a collection of facilities while providing a single interface to the utility or ISO for the management and billing of those facilities.

- DRAS Client: This is a sub-system within the facility or aggregator that is responsible for bridging communications between the DRAS and any automated system (e.g., EMCS) that is responsible for controlling energy consumption. It may be a software-based DRAS Client that is implemented with an existing subsystem such as the EMCS or it may be a dedicated piece of hardware whose only responsibility is to proxy communications between the DRAS and EMCS.

It is the goal of the OpenADR specification that the DRAS communications are independent of the platform and technologies in which the DRAS Client is implemented and that the DRAS supports a range of DRAS Clients with different capabilities. It is not within the scope of this document to specify how the DRAS Client interfaces and communicates with the facility EMCS or IT systems. The DRAS will support two classes of DRAS Clients henceforth referred to as "Intelligent" DRAS Clients and "Simple" DRAS Clients. Intelligent DRAS Clients are typically software based and are able to make sophisticated decisions based upon the information available. Therefore Intelligent DRAS Clients should receive all available information concerning DR events generated from the Utilities. On the other hand Simple DRAS Clients have little or no capabilities for making decisions are typically just hardwired to existing EMCS systems via relays. As such they receive very simple signal waveforms associated with DR events such as high, medium, and normal which get mapped to relay contacts. The mapping between the utility or ISO generated DR events and the signal levels are performed in the DRAS before being sent to the DRAS Client.

\section{D.1.1.2 Actions}

Actions are depicted as ovals and are tasks that are performed by a role within a system (Figure D2).

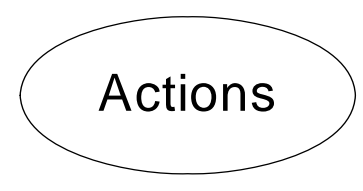

Figure D2. Use Case Actions 


\section{D.1.1.3 Roles}

Typically, a role (or an actor in a role) is a member of a system and is responsible for performing actions on a system. The relationship between a role and an action is depicted by a line from the role to the action in the diagram. A role may be filled by a human, or by some hardware or software entity. Human roles are depicted differently from machine roles as shown in Figure D3.

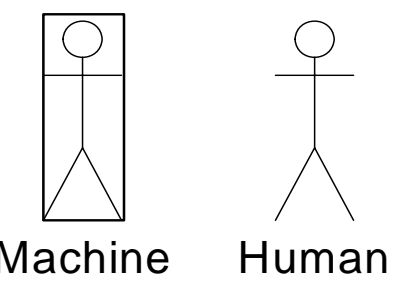

Figure D3. Use Case Roles

\section{D.2 Specific Use Cases}

The following sections outline some specific use cases of DR programs currently being offered by the utilities or ISOs.

\section{D.2.1 Critical Peak Pricing (CPP)}

Critical Peak Pricing (CPP) is a DR program that is currently offered by all three California investor owned utilities (IOUs.) Pacific Gas \& Electric's (PG\&E's) website, http://www.pge.com/biz/demand response/critical peak pricing/, provides the following description of a PG\&E CPP program:

CPP events will generally be triggered by temperature, but may also be activated by PG\&E as warranted by extreme system conditions:

- Special alerts issued by the California Independent System Operator

- Under conditions of high forecasted California spot market power prices

- For testing or evaluation purposes

\section{D.2.1.1 CPP Directly to Facility}

The following set of use cases concern CPP programs which are enacted directly with participant facilities. The use case diagram can be seen in Figure D4. 


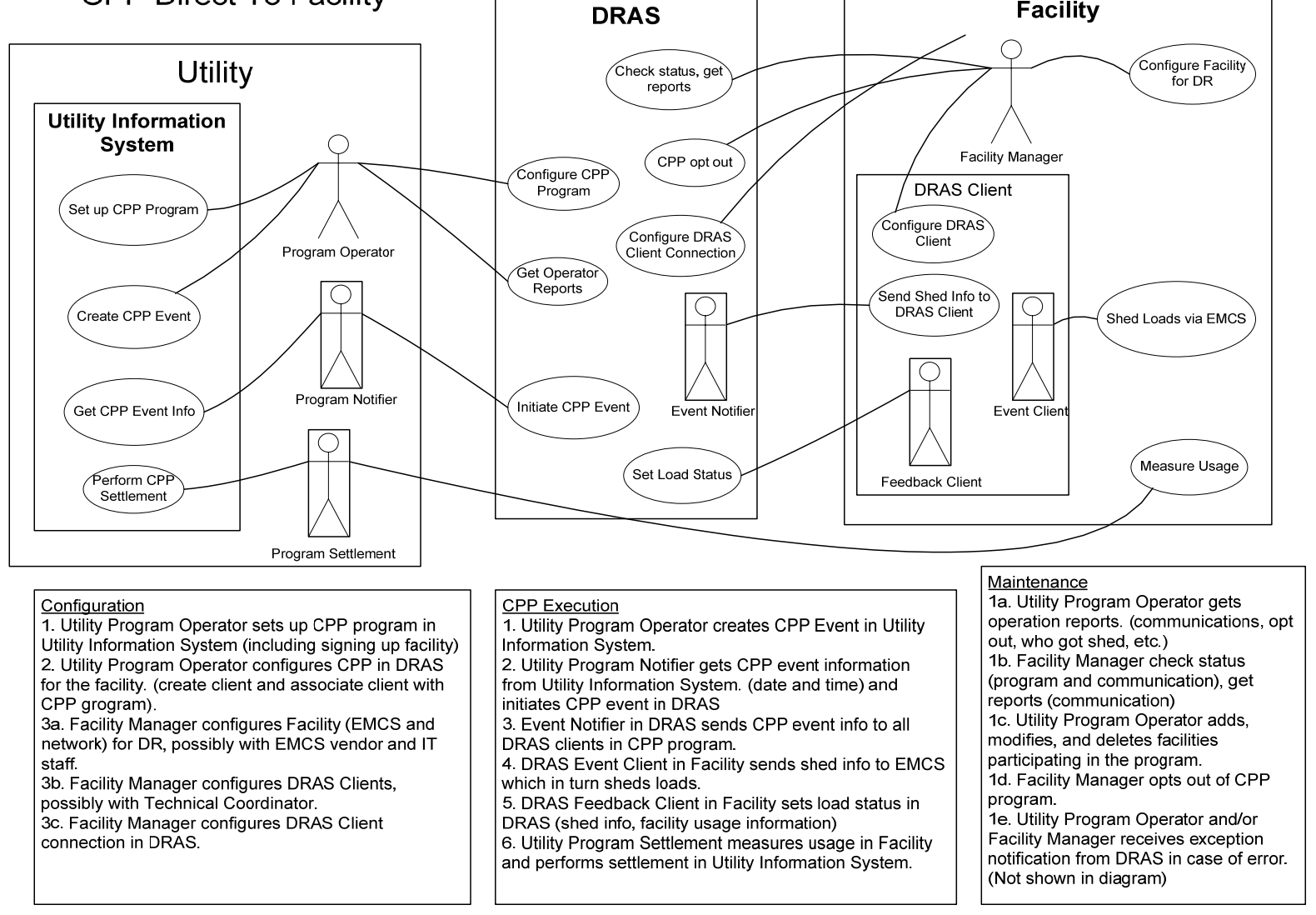

Figure D4. CPP Direct To Facility

\section{D.2.1.1.1 CPP Configuration}

This includes entering all the information necessary for the participant to participate in the CPP DR program and involves the following actions.

1. The utility program operator sets up the CPP program in the Utility Information System. This includes signing up participants and entering all required information necessary for the participant to participate in the CPP program into the UIS. The details of this process are beyond the scope of this document.

2. The utility program operator configures the CPP in the DRAS for the facility. This includes entering information into the DRAS to allow the facility operator to access the DRAS and set up their DRAS Client so that it may communicate with the DRAS. It includes entering the following information:

- Program definition

o Event launching endpoint

o Program to event to the DRAS Client signal mapping

- Utility assigned account number used for settlement

- Customer identification

- Customer password 
- Geographic location

- Grid location

3a. The facility manager configures the facility's EMCS and network for DR, possibly in conjunction with the EMCS vendor and IT staff. This could include programming the EMCS to respond to DR events and shed or shift loads appropriately. The details of this process are beyond the scope of this document.

$3 \mathrm{~b}$. The facility manager configures DRAS Clients, possibly in conjunction with the technical coordinator. DRAS Clients may take many forms, both in terms of hardware and software. This step configures the DRAS Client so that it can communicate with the Facilities systems that are responsible for managing the loads. The details of this process are beyond the scope of this document.

3c. The facility manager configures DR program parameters and DRAS Client connection in the DRAS. This step establishes the connection between the DRAS Client and the DRAS. Typically this includes the following types of information:

- Identification and password of the Customer participating in the program

- Contact information, i.e. phone number, pager, email, etc.

- DRAS Client communications parameters

o DRAS IP address

0 identification

o password

o IP connection information

o polling frequency if polling the DRAS Client

- Optionally - Load reduction potential (per time block per level)

- Exception parameters

- Opt-out dates

Note that this action is currently depicted as occurring in the DRAS, but depending upon how this step is subsequently implemented in future OpenADR standards, this may be performed in the DRAS Client and not in the DRAS.

\section{D.2.1.1.2 CPP Execution}

The actions to execute CPP events consist of the following steps:

1. The utility program operator creates the CPP event in the Utility Information System. In this step a program operator schedules the CPP event in the Utility Information System. The details of this process are beyond the scope of this document.

2. The utility program notifier gets the CPP event information from the Utility Information System and initiates the CPP event in the DRAS. The information sent to the DRAS by the utility program notifier sub-system includes: 
- Program type

- Date and time of the event

- Date and time issued

- Geographic location

- Customer list (account numbers)

3. The event notifier in the DRAS sends the CPP event information to all DRAS Clients in the CPP program. The CPP event information sent to the DRAS Clients includes:

- Utility event information for Intelligent DRAS Clients

o Date and time of the event

o Date and time issued

o Geographic location (optional)

o Mode and pending signals

- Mode signal levels for Simple DRAS Clients (e.g. normal, moderate, high)

- Event pending signal for Simple DRAS Clients (e.g. yes/no, or simple quantification of how far in advance notice is to be sent)

4. The DRAS Event Client for the facility sends shed or shift information to the EMCS which in turn sheds loads. The details of this process are beyond the scope of this document.

5. The DRAS Feedback Client for the facility sends the system load status to the DRAS. This is a feedback mechanism that is used to record how the facility responded to the DR event. It includes the following information:

- Program identifier

- Facility identifier

- Date and time of the event (shed or shift)

- Shed data in $\mathrm{kW} / \mathrm{kWh}$

- Load reduction end uses (HVAC, lighting, etc.)

6. The utility program settlement measures usage in the facility and performs settlement in the Utility Information System. How this process is done is beyond the scope of this document.

\section{D.2.1.1.3 CPP Maintenance}

This scenario consists of a set of actions which are necessary to maintain the CPP program. Unlike the configuration and execution scenarios this set of actions are less a prescribed set of steps and more a set of possible actions that may be performed by the various roles at unrelated times.

1a. The utility program operator gets the operation reports. The utility program operator can get the following status information from the DRAS at any time: 
- Event status (for all participants)

o current outstanding events

- Load reduction potential based upon all customers in program (optional)

- Feedback from DRAS Clients (for those that have optional feedback)

o event logs

$1 \mathrm{~b}$. The facility manager checks the status. The utility program operator can get the following status information from the DRAS at any time:

- DRAS Client communications status

o current status

o last contact

o current signals levels

o current customer manual control levels (opt-out)

o communication logs

o signal logs

o manual control logs

- Event status (same as above)

1c. The utility program operator adds, modifies and deletes facilities participating in the program. This is similar to the original configuration step.

1d. The facility manager opts out of the DR program. At any time, the facility manager can opt-out of the DR program on the DRAS. When in an opt-out condition, DR events are not propagated to the DRAS Client. The opt-out can be either for the entire program or a single event or a specific event-mode (e.g. normal, moderate, high)

1e. The utility program operator and/or the facility manager receives an exception notification from the DRAS in case of an error (this is not shown in the diagram). When an error condition occurs in the DRAS which may require some sort of action by either the participant or the utility the DRAS will send a message to the respective operators via email, voice or pager. Note that this alarming interface does not cover exception conditions that are part of the DRAS operation and managed by the DRAS operator, which are outside the scope of this document. The type of exceptions covered by this interface includes:

- DRAS Client communications failure.

\section{D.2.1.2 CPP via Aggregator}

In the actual CPP programs currently offered by PG\&E, the aggregators do not typically play a role. The following use cases envision how an aggregator might play a role with the DRAS. It is very similar to the direct to facility use case. The use case diagram can be seen in Figure D5. 


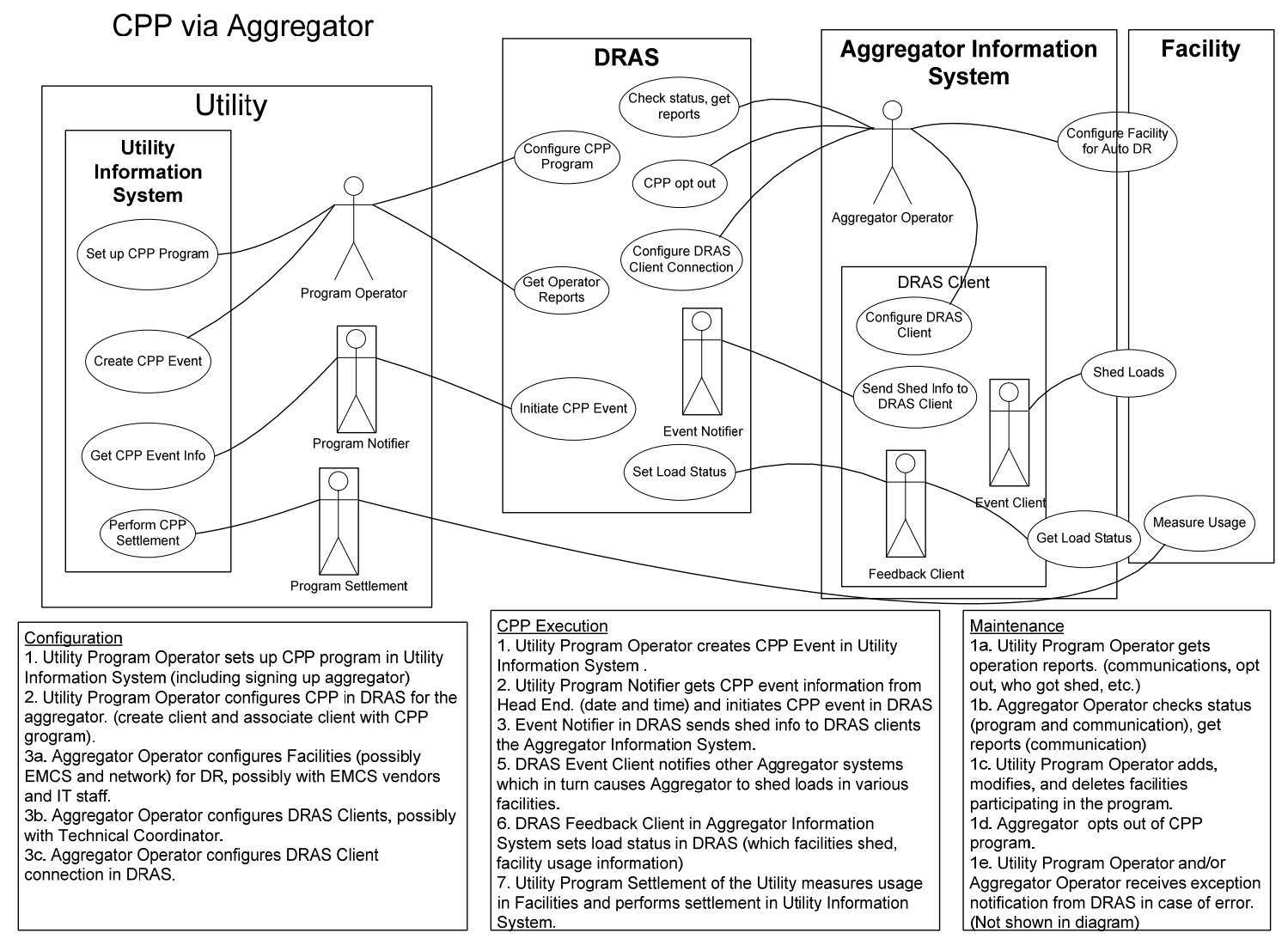

Figure D5. CPP via Aggregator

\section{D.2.1.2.1 CPP Configuration}

This includes entering all the information necessary for the participant to participate in the CPP DR program and involves the following actions.

1. The utility program operator sets up the CPP program in the Utility Information System. This includes signing up participants and entering all required information necessary for the participant to participate in the CPP program into the UIS. The details of this process are beyond the scope of this document.

2. The utility program operator configures the CPP in the DRAS for the facility. This includes entering information into the DRAS to allow the facility operator to access the DRAS and set up their DRAS Client so that it may communicate with the DRAS. It includes entering the following information:

- Program definition

o Event launching endpoint

o Program to event to DRAS Client signal mapping

- Utility assigned account number used for settlement

- Customer identification

- Customer password 
- Geographic location

- Grid location

3a. The aggregator configures the facility (EMCS and network) for DR, possibly in conjunction with the EMCS vendor and IT staff. The details of this process are beyond the scope of this document.

$3 \mathrm{~b}$. The aggregator configures the DRAS Clients, possibly in conjunction with the technical coordinator. DRAS Clients may take many forms, both in terms of hardware and software. This step configures the DRAS Client so that it can communicate with the aggregator's systems that are responsible for managing the loads. The details of this process are beyond the scope of this document.

3c. The aggregator configures the DR program parameters and the DRAS Client connection in the DRAS. This step establishes the connection between the DRAS Client and the DRAS. Typically this includes the following types of information:

- Identification and password of the Customer participating in the program.

- Contact information, i.e. phone number, pager, email, etc.

- DRAS Client communications parameters.

o DRAS IP address

o identification

0 password

o IP connection information

o polling frequency if a polling the DRAS Client.

- Optionally - Load reduction potential (per time block per level)

- Exception parameters.

- Opt-out dates

Note that this action is currently depicted as occurring within the DRAS, but depending upon how this step is subsequently implemented in a future OpenADR standards, this may be performed within the DRAS Client and not within the DRAS.

\section{D.2.1.2.2 CPP Execution}

The set of actions to execute CPP events include the following steps:

1. The utility program operator creates CPP event in the Utility Information System. In this step a program operator schedules a CPP event in the Utility Information System. The details of this process are beyond the scope of this document.

2. The utility program notifier gets CPP event information from the Utility Information System and initiates CPP event in the DRAS. The information sent to the DRAS by the utility program notifier sub-system includes the following information: 
- Program type

- Date and time of the event

- Date and time issued

- Geographic location

- Customer list (account numbers)

3. The event notifier in the DRAS sends CPP event information to all DRAS Clients in CPP program. The CPP event information sent to the DRAS Clients includes the following:

- Utility event information for Intelligent DRAS Clients

o Date and time of the event

o Date and time issued

- Geographic location (optional)

o Mode and pending signals

- Mode signal levels for Simple DRAS Clients (e.g. normal, moderate, high)

- Event pending signal for Simple DRAS Clients (e.g. yes/no, or simple quantification of how far in advance notice is to be sent)

4. The DRAS Event Client for the facility sends shed or shift information to the aggregator's system which in turn causes the aggregator to shed or shift loads in various facilities. How this process is done is beyond the scope of this document.

5. The DRAS Feedback Client for the aggregator's system sends the system load status to the DRAS. This is a feedback mechanism that is used to record how the facility responded to the DR event. It includes the following information:

- Program identifier

- Facility identifier

- Date and time of the event (shed or shift)

- Shed data in $\mathrm{kW} / \mathrm{kWh}$

- Load reduction end uses (HVAC, lighting, etc.)

6. The utility program settlement measures usage in the facility and performs settlement in the Utility Information System. The details of this process are beyond the scope of this document.

\section{D.2.1.2.3 CPP Maintenance}

This scenario consists of a set of actions which are necessary to maintain the CPP program. Unlike the Configuration and Execution scenarios this set of actions are less a prescribed set of steps and more a set of possible actions that may be performed by the various roles at unrelated times.

1a. The utility program operator gets the operation reports. The utility program operator can get the following status information from the DRAS at any time: 
- Event status (for all participants)

o current outstanding events

- Load reduction potential based upon all customers in program (optional)

- Feedback from DRAS Clients (for those that have optional feedback)

o event logs

$1 b$. The facility manager checks status. The utility program operator can get the following status information from the DRAS at any time:

- DRAS Client communications status

o current status

o last contact

o current signals levels

o current customer manual control levels (opt-out)

o communication logs

o signal logs

o manual control logs

- Event status (same as above)

1c. The utility program operator adds, modifies and deletes facilities participating in the program. This is similar to the original configuration step.

$1 \mathrm{~d}$. The aggregator opts out of the DR program. At any time, the aggregator or the aggregator acting as facility manager can opt out of the DR program on the DRAS. When there is an opt-out condition, DR events are not propagated to the DRAS Client. The opt-out can be either for the entire program or a single event.

1e. The utility program operator and/or the aggregator operator receive an exception notification from the DRAS in case of an error (this is not shown in the diagram). When an error condition occurs in the DRAS which may require some sort of action by either the participant or the utility the DRAS will send a message to the respective operators via email, voice or pager. Note that this alarming interface does not cover exception conditions that are part of the DRAS operation and managed by the DRAS operator, which is outside the scope of this document. The types of exceptions covered by this interface include:

- DRAS Client communications failure.

\section{D.2.2 Demand Bidding Program (DBP)}

The Demand Bidding Program (DBP) is offered by PG\&E. PG\&E's website, http://www.pge.com/mybusiness/energysavingsrebates/demandresponse/dbp/, has the following description of this program: 
The (Automated) Demand Bidding Program (E-DBP) pays an incentive to reduce electric loads according to a voluntary bid made for a scheduled load reduction on the following non-holiday weekday. Under this program, participants receive a credit equal to the product of the qualified kilowatt $(\mathrm{kW})$ energy reduction and the incentive price of up to $\$ 0.60 / \mathrm{kWh}$.

\section{How the Day-Ahead Program Works}

When the forecasted system reserve margins for the next day result in the California Independent System Operator (CAISO) issuing an Alert Notice, or when the CAISO day-ahead forecasted peak demand is 43,000 megawatts (MW) or greater, or when Pacific Gas and Electric Company (PG\&E), in its sole opinion, forecasts that resources will not be adequate, PG\&E may request load reduction bids from customers for the following non-holiday weekday. Customers seeking to participate in the E-DBP can submit bids for a proposed level of curtailment.

Participating customers will have until 12:00 noon on the day before a proposed curtailment event to submit bids via the ITRON's Inter-Act website. Upon evaluation from Pacific Gas and Electric Company, customers will be notified of bid acceptance after 4 p.m. of the same day. Unless a specific megawatt (MW) limit is requested, PG\&E will accept all bids. Participants must bid a minimum of two consecutive hours throughout the day and must meet the minimum energy reduction threshold of 50 kilowatts $(\mathrm{kW})$ for single account and 200 kilowatts $(\mathrm{kW})$ for aggregated accounts.

\section{Day-Ahead Incentives}

The Day-Ahead E-DBP incentives are calculated on an hourly basis, and will be equal to the product of the qualified $\mathrm{kW}$ reduction for each hour a bid was accepted and the incentive price of $\$ 0.50 / \mathrm{kWh}$.

\section{How the Day-Of Program Works}

When the CAISO issues any alert during the day reflecting stress on the system, PG\&E may implement an E-DBP event for that same day. Customers have one hour after a Day-Of event has been issued to submit bids for a proposed level of curtailment. Unless a specific megawatt (MW) limit is requested, PG\&E will accept all bids.

If a customer already has accepted-bids for the Day-Ahead program customers may 1) increase its bids for those hours that the Day-Ahead and Day-Of DBP events coincide, and 2) submit new bids for those hours in the Day-Of DBP event that were not a part of the Day-Ahead event. If no adjustments to the bid are made the participation is automatically transferred to the Day-Of program with the higher incentive price. 


\section{Day-Of Incentives}

The Day-Of E-DBP incentives are calculated on an hourly basis, and will be equal to the product of the qualified $\mathrm{kW}$ reduction for each hour a bid was accepted and the incentive price of $\$ 0.60 / \mathrm{kWh}$.

\section{D.2.2.1 DBP Directly to Facilities}

The following set of use cases concern CPP programs which are enacted directly with participant facilities. The use case diagram can be seen in Figures D6 and D7.

\section{DB Program Configuration}

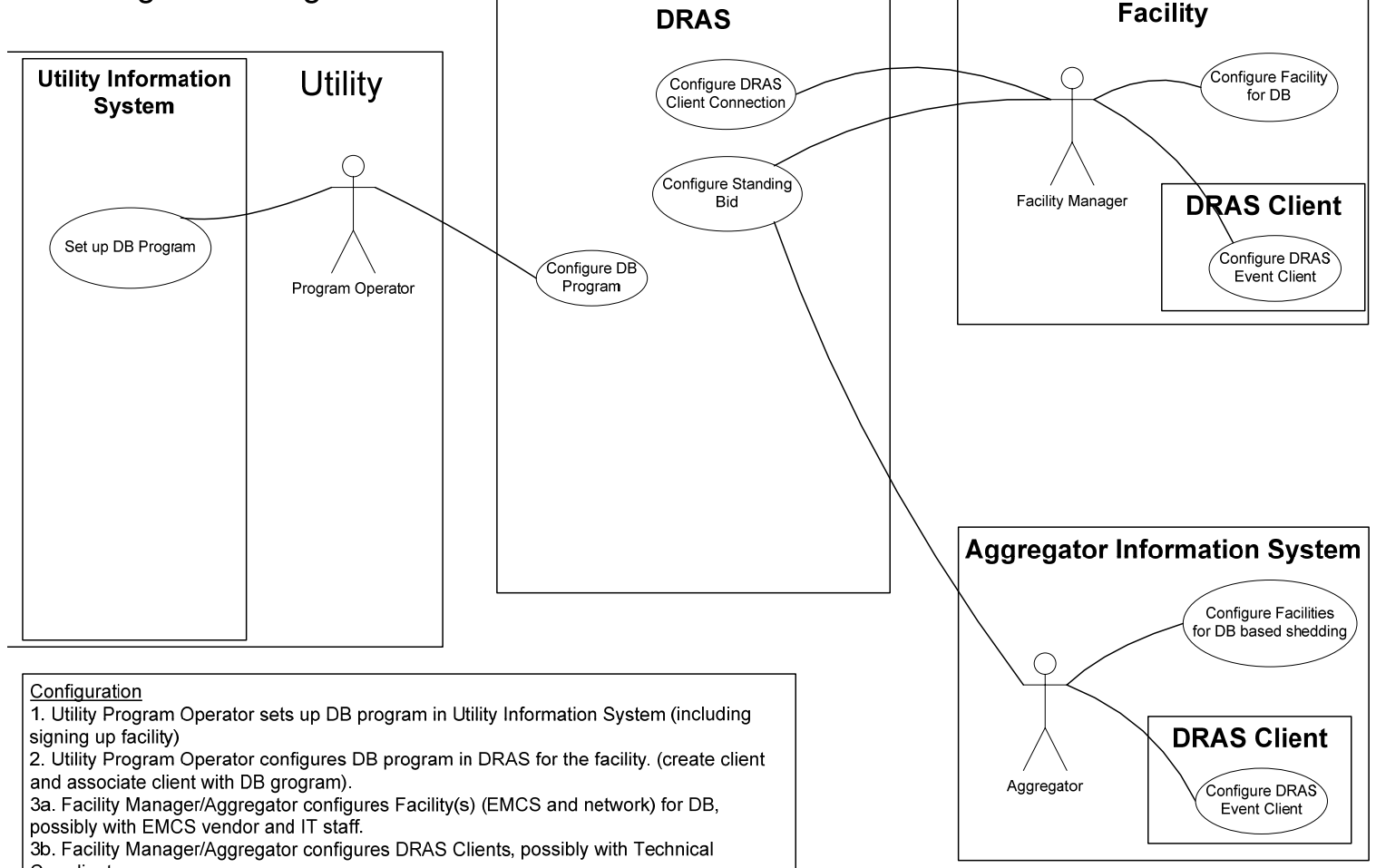

Coordinator.

3c. Facility Manager/Aggregator sets standing bid in the DRAS

3d. Facility Manager/Aggregator configures DRAS Client connection in DRAS.

Figure D6. DB Program Configuration 
DBP Direct To Facility

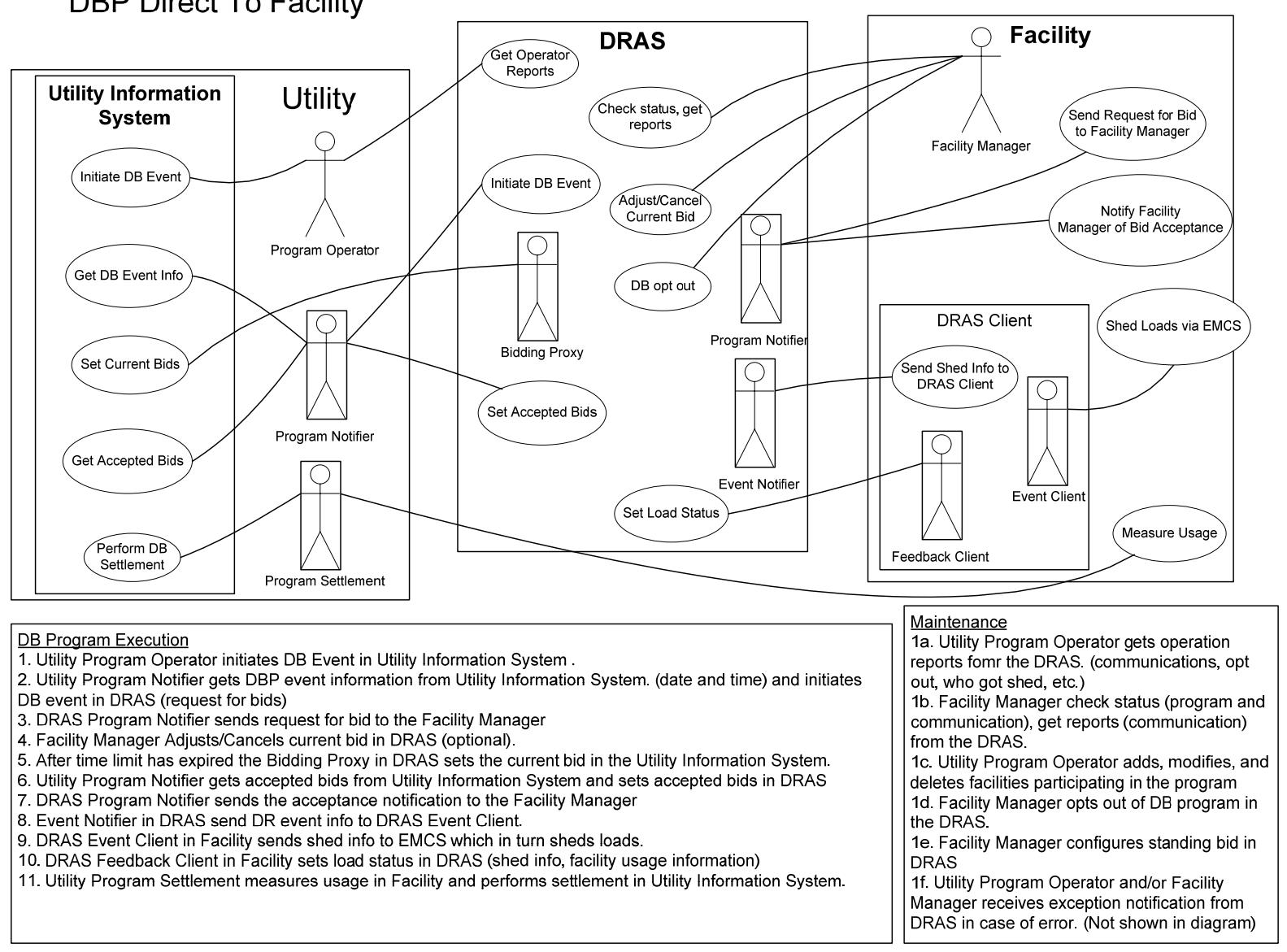

\section{Figure D7. DBP Direct to Facility}

\section{D.2.2.1.1 DBP Configuration}

This includes entering all the information necessary for the participant to participate in the DBP DR program and involves the following actions.

1. The utility program operator sets up the DBP program in the Utility Information System. This includes signing up participants and entering all the required information necessary for the participant to participate in the DBP program into the UIS. The details of this process are beyond the scope of this document.

2. The utility program operator configures the DBP in the DRAS for the facility. This includes entering information into the DRAS to allow the facility operator to access the DRAS and set up their DRAS Client so that it may communicate with the DRAS. It includes entering the following information:

- Program definition

o Event launching endpoint

o Program to event to DRAS Client signal mapping

- Utility assigned account number used for settlement

- Customer identification 
- Customer password

- Geographic location

- Grid location

3a. The facility manager configures the facility (EMCS and network) for DR, possibly in conjunction with the EMCS vendor and IT staff. This could include programming the EMCS to respond to DR events and shed or shift loads appropriately. The details of this process are beyond the scope of this document.

$3 b$. The facility manager configures the DRAS Clients, possibly in conjunction with the technical coordinator. DRAS Clients may take many forms, both in terms of hardware and software. This step configures the DRAS Client so that it can communicate with the Facilities systems that are responsible for managing the loads. The details of this process are beyond the scope of this document.

3c. The facility manager configures a standing bid in the DRAS. This is the bid that will be automatically placed by the DRAS when a request for bids comes from the utility. It includes the following:

- Load reduction bids per time block (price and load amount)

$3 \mathrm{~d}$. The facility manager configures the DR program parameters and DRAS Client connection in the DRAS. This step establishes the connection between the DRAS Client and the DRAS. Typically this includes the following types of information:

- Identification and password of the Customer participating in the program.

- Contact information, i.e. phone number, pager, email, etc.

- DRAS Client communications parameters.

o DRAS IP address

o identification

o password

o IP connection information

o polling frequency if a polling DRAS Client.

- Optionally-Load reduction potential (per time block per level)

- Exception parameters.

- Opt-out dates

Note that this action is currently depicted as occurring in the DRAS, but depending upon how this step is subsequently implemented in a future OpenADR standards, this may be performed in the DRAS Client and not in the DRAS. 


\section{D.2.2.1.2 DBP Execution}

The set of actions to execute DBP events include the following steps:

1. The utility program operator creates or schedules the DBP event in the Utility Information System. The details of this process are beyond the scope of this document.

2. The utility program notifier gets the DBP event information from the Utility Information System and initiates a DBP event in the DRAS. The information sent to the DRAS by the utility program notifier sub-system includes the following information:

- Program type

- Date and time of the event

- Date and time issued

- Geographic location

- Customer list (account numbers)

- Request For Bids (RFB) issue date and time

- RFB close time

- Price offered for load reduction per time block

3. The DRAS program notifier sends request for bid to the facility manager. This notification typically comes in the form of an email, phone call or page.

4. The facility manager adjusts or cancels their current bid in the DRAS. This is an optional step and allows the Manager to adjust their bid for that particular event. If this step is not performed then the DRAS will submit the standing bid after some period of time.

5. After time limit has expired the Bidding Proxy in the DRAS sets the current bid in the Utility Information System. The information sent by the DRAS includes the following:

- Customer account number

- Load reduction bids per time block

6. The utility program notifier gets accepted bids from the Utility Information System and sets accepted bids in the DRAS. The accepted bids form the set of participants that the DRAS will send DB shed or shift events to. The information concerning accepted bids include:

- Customer list (account number)

- Accept or reject

- Load reduction bids per time block (for verification)

7. The DRAS program notifier sends the acceptance notification to the facility manager. The manager is notified of an accepted bid via phone, email, or page. 
8. The event notifier in the DRAS sends the DBP event information to all DRAS Clients whose bids were accepted. The DBP event information sent to the DRAS Clients includes the following:

- Utility event information for Intelligent DRAS Clients

o Date and time of the event

o Date and time issued

- Geographic location (optional)

o Mode and pending signals

- Mode signal levels for Simple DRAS Clients (e.g. normal, moderate, high)

- Event pending signal for Simple DRAS Clients (e.g. yes/no, or simple quantification of how far in advance notice is to be sent)

9. The DRAS Event Client in the facility sends shed or shift information to the EMCS which in turn causes loads to be shed or shift. How this process is done is beyond the scope of this document.

10. The DRAS Feedback Client in the aggregator's system sends the system load status information to the DRAS. This is a feedback mechanism that is used to record how the facility responded to the DR event. It includes the following information:

- Program identifier

- Facility identifier

- Date and time of the event (shed or shift)

- Shed data in $\mathrm{kW} / \mathrm{kWh}$

- Load reduction end uses (HVAC, lighting, etc.)

- Event Type (Day-Ahead or Day-Of)

11. The utility program settlement measures usage in the facility and performs settlement in the Utility Information System. How this process is done is beyond the scope of this document.

\section{D.2.2.1.3 DBP Maintenance}

This scenario consists of a set of actions which are necessary to maintain the DBP program. Unlike the configuration and execution scenarios this set of actions are less a prescribed set of steps and more a set of possible actions that may be performed by the various roles at unrelated times.

1a. The utility program operator gets the operation reports. The utility program operator can get the following status information from the DRAS at any time:

- Event status (for all participants)

o current outstanding events

- Load reduction potential based upon all customers in program (optional) 
- Feedback from DRAS Clients (for those that have optional feedback) o event logs

- Bids and signal levels per time block for current events (for all participants, indivually and grouped together)

1b. The facility manager checks status. The facility manager can get the following status information from the DRAS at any time:

- DRAS Client communications status

o current status

o last contact

o current signals levels

o current customer manual control levels (opt-out)

o communication logs

o signal logs

o manual control logs

- Event status (same as above)

- Bids and signal levels per time block for current events

1c. The utility program operator adds, modifies or deletes facilities participating in the program. This is similar to the original configuration step.

1d. The facility manager opts out of the DR program. At any time, the facility manager can opt-out of the DR program on the DRAS. When there is an opt-out condition, DR events are not propagated to the DRAS Client. The opt-out can be either for the entire program or a single event.

1e. The facility manager adjusts standing bid in the DRAS.

1f. The utility program operator and/or the facility manager receive an exception notification from the DRAS in case of an error (this is not shown in the diagram). When an error condition occurs in the DRAS which may require some sort of action by either the participant or the utility the DRAS will send a message to the respective operators via email, voice or pager. Note that this alarming interface does not cover exception conditions that are part of the DRAS operation and managed by the DRAS operator, which is outside the scope of this document. The types of exceptions covered by this interface include:

- DRAS Client Communications Failure 


\section{D.2.2.2 DBP via Third Party Aggregators}

The following set of use cases concern DBP programs which are enacted with aggregators. The use case diagram can be seen in Figure D8.

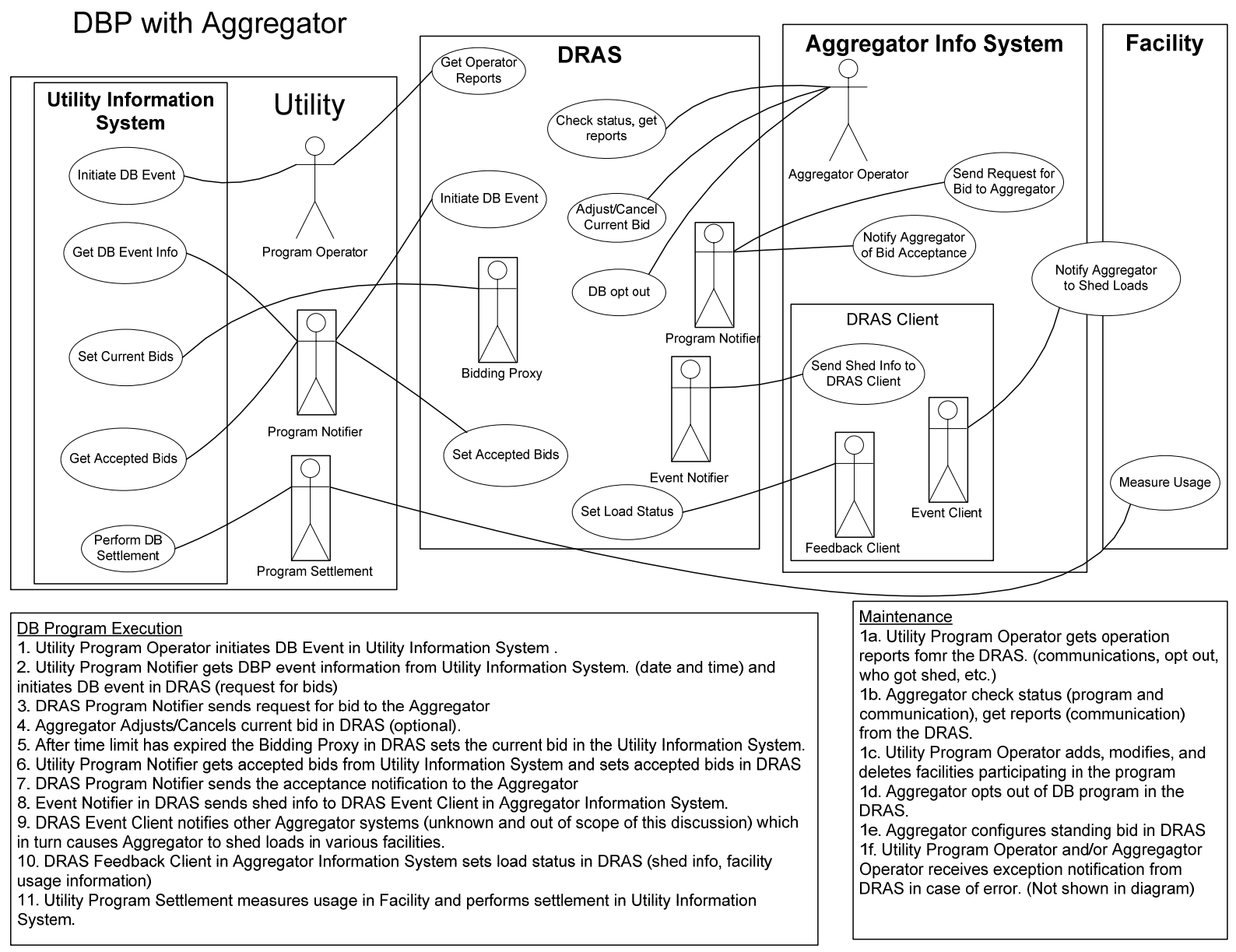

\section{Figure D8. DBP with Aggregator}

\section{D.2.2.2.1 DBP Configuration}

This includes entering all the information necessary for the participant to participate in the DBP DR program and involves the following actions:

1. The utility program operator sets up the DBP program in the Utility Information System. This includes signing up participants and entering all required information necessary for the participant to participate in the DBP program into the UIS. The details of this process are beyond the scope of this document.

2. The utility program operator configures the DBP in the DRAS for the facility. This includes entering information into the DRAS to allow the facility operator to access the DRAS and set up their DRAS Client so that it may communicate with the DRAS. It includes entering the following information: 
- Program definition

- Event launching endpoint

o Program to event to DRAS Client signal mapping

- Utility assigned account number used for settlement

- Customer identification

- Customer password

- Geographic location

- Grid location

3a. The aggregator configures their systems for DR. The details of this process are beyond the scope of this document.

$3 \mathrm{~b}$. The aggregator configures the DRAS Clients, possibly in conjunction with the technical coordinator. DRAS Clients may take many forms, both in terms of hardware and software. This step configures the DRAS Client so that it can communicate with the aggregator systems that are responsible for managing the loads. The details of this process are beyond the scope of this document.

3c. The aggregator sets their standing bid in the DRAS. This is the bid that will be automatically placed by the DRAS when a request for bids comes from the utility. It includes the following:

Load reduction bids per time block (price and load amount)

$3 \mathrm{~d}$. The aggregator configures the DR program parameters and the DRAS Client connection in the DRAS. This step establishes the connection between the DRAS Client and the DRAS. Typically this includes the following types of information:

- Identification and password of the Customer participating in the program.

- Contact information, i.e. phone number, pager, email, etc.

- DRAS Client communications parameters.

o DRAS IP address

o identification

o password

o IP connection information

o polling frequency if a polling DRAS Client.

- Optionally - Load reduction potential (per time block per level)

- Exception parameters.

- Opt-out dates

Note that this action is currently depicted as occurring in the DRAS, but depending upon how this step is subsequently implemented in a future OpenADR standards, this may be performed in the DRAS Client and not in the DRAS. 


\section{D.2.2.2.2 DBP Execution}

The set of actions to execute DBP events include the following steps:

1. The utility program operator creates the DBP event in the Utility Information System. In this step a program operator schedules a DBP event in the Utility Information System. The details of this process are beyond the scope of this document.

2. The utility program notifier gets DBP event information from the Utility Information System and initiates the DBP event in the DRAS. The information sent to the DRAS by the utility program notifier sub-system includes the following information:

- Program type

- Date and time of the event

- Date and time issued

- Geographic location

- Customer list (account numbers)

- Request For Bids (RFB) issue date and time

- $\quad$ RFB close time

- Price offered for load reduction per time block

3. The DRAS program notifier sends a request for bid to the aggregator. This notification typically comes in the form of an email, phone call or page.

4. The aggregator adjusts or cancels their current bid in the DRAS. This is an optional step and allows the Manager to adjust their bid for that particular event. If this step is not performed then the DRAS will submit the standing bid after some pre-set period of time.

5. After the time limit has expired, the Bidding Proxy in the DRAS sets the current bid in the Utility Information System. The information sent by the DRAS includes the following:

- Customer account number

- Load reduction bids per time block

6. The utility program notifier gets accepted bids from the Utility Information System and sets accepted bids in the DRAS. The accepted bids form the set of participants that the DRAS will send DB shed or shift events to. The information concerning accepted bids include:

- Customer list (account number)

- Accept or reject

- Load reduction bids per time block (for verification)

7. The DRAS program notifier sends the acceptance notification to the facility manager. The manager is notified of an accepted bid via phone, email, or page. 
8. The event notifier in the DRAS sends DBP event information to all DRAS Clients whose bids were accepted. The DBP event information sent to the DRAS Clients includes the following:

- Utility event information for Intelligent DRAS Clients

o Date and time of the event

o Date and time issued

- Geographic location (optional)

o Mode and pending signals

- Mode signal levels for Simple DRAS Clients (e.g. normal, moderate, high)

- Event pending signal for Simple DRAS Clients (e.g. yes/no, or simple quantification of how far in advance notice is to be sent)

9. The DRAS Event Client for the facility sends shed or shift information to the aggregator's system which in turn causes the aggregator to shed or shift loads in various facilities. How this process is done is beyond the scope of this document.

10. The DRAS Feedback Client for the aggregator's system sends the system load status to the DRAS. This is a feedback mechanism that is used to record how the facility responded to the DR event. It includes the following information:

- Program identifier

- Facility identifier

- Date and time of the event (shed or shift)

- Shed data in $\mathrm{kW} / \mathrm{kWh}$

- Load reduction end uses (HVAC, lighting, etc.)

- Event Type (Day-Ahead or Day-Of)

11. The utility program settlement measures usage in the facility and performs settlement in Utility Information System. How this process is done is beyond the scope of this document.

\section{D.2.2.2.3 DBP Maintenance}

This scenario consists of a set of actions which are necessary to maintain the DBP program. Unlike the Configuration and Execution scenarios this set of actions are less a prescribed set of steps and more a set of possible actions that may be performed by the various roles at unrelated times.

1a. The utility program operator gets operation reports. The utility program operator can get the following status information from the DRAS at any time:

- Event status (for all participants)

o current outstanding events

- Load reduction potential based upon all customers in program (optional) 
- Feedback from DRAS Clients (for those that have optional feedback) o event logs

- Bids and signal levels per time block for current events (for all participants, indivually and grouped together)

1b. The aggregator checks status. The aggregator can get the following status information from the DRAS at any time:

- DRAS Client communications status

o current status

o last contact

o current signals levels

o current customer manual control levels (opt-out)

o communication logs

o signal logs

o manual control logs

- Event status (same as above)

- Bids and signal levels per time block for current events

1c. The utility program operator adds, modifies and deletes facilities participating in the program. This is similar to the original configuration step.

$1 \mathrm{~d}$. The aggregator opts out of the DR program. At any time, the aggregator or the aggregator acting as facility manager can opt out of the DR program on the DRAS. When there is an opt-out condition, DR events are not propagated to the DRAS Client. The opt-out can be either for the entire program or a single event.

1e. The aggregator adjusts standing bid in the DRAS.

1f. The utility program operator and/or the aggregator receive an exception notification from the DRAS in case of an error (this is not shown in the diagram). When an error condition occurs in the DRAS which may require some sort of action by either the participant or the utility the DRAS will send a message to the respective operators via email, voice or pager. Note that this alarming interface does not cover exception conditions that are part of the DRAS operation and managed by the DRAS operator, which is outside the scope of this document. The types of exceptions covered by this interface include:

- DRAS Client communications failure.

\section{D.2.3 Capacity Bidding Program (CBP)}

The Capacity Bidding Program (CBP) is offered by PG\&E. PG\&E's website, http://www.pge.com/mybusiness/energysavingsrebates/demandresponse/cbp/, provides the following description of this program: 
The Capacity Bidding Program (E-CBP) is a mandatory program that pays you a monthly incentive to reduce your load to a pre-determined amount when an electric resource generation facility reaches or exceeds heat rates of 15,000 BTU/kWh. E-CBP operates seasonally from May 1 to October 31.

\section{How It Works}

To participate in the program, you must simply identify which of the two option best suites you and your business line - Day Ahead or Day Of program hours are 11:00 a.m. to 7:00 p.m., Monday through Friday excluding Pacific Gas and Electric Company holidays and weekends.

\section{Day-Ahead Curtailment}

- Maximum of one event per day

- Will not exceed 24 events per month

- Notices will be issued by 3:00 p.m. on the business day before the operation day.

\begin{tabular}{|c|c|c|c|c|c|c|}
\hline \multicolumn{6}{|l|}{ Directly-Enrolled Customer in Day-Ahead Option } \\
\hline Product & May & June & July & August & September & October \\
\hline $1-4$ & $\$ 0.00 / \mathrm{kW}$ & $\$ 2.97 / \mathrm{kW}$ & $\$ 12.48 / \mathrm{kW}$ & $\$ 17.26 / \mathrm{kW}$ & $\$ 10.64 / \mathrm{kW}$ & $\$ 0.00 / \mathrm{kW}$ \\
\hline $2-6$ & $\$ 0.00 / \mathrm{kW}$ & $\$ 2.97 / \mathrm{kW}$ & $\$ 12.48 / \mathrm{kW}$ & $\$ 17.26 / \mathrm{kW}$ & $\$ 10.64 / \mathrm{kW}$ & $\$ 0.00 / \mathrm{kW}$ \\
\hline $4-8$ & $\$ 0.00 / \mathrm{kW}$ & $\$ 2.97 / \mathrm{kW}$ & $\$ 12.48 / \mathrm{kW}$ & $\$ 17.26 / \mathrm{kW}$ & $\$ 10.64 / \mathrm{kW}$ & $\$ 0.00 / \mathrm{kW}$ \\
\hline Aggregators in Day-Ahead Option & & & & & \\
\hline
\end{tabular}

\section{Day-Of Curtailment}

- Maximum of one event per day

- Will not exceed 24 events per month

- Notifies customer 30 minutes before the CAISO Hour Ahead Market closes (about 3 before the operation event hour starts) 


\begin{tabular}{|c|c|c|c|c|c|c|}
\hline \multicolumn{7}{|c|}{ Aggregators in Day-Of Option } \\
\hline Product & May & June & July & August & September & October \\
\hline $1-4$ & $\$ 0.00 / \mathrm{kW}$ & $\$ 3.42 / \mathrm{kW}$ & $\$ 14.35 / \mathrm{kW}$ & $\$ 19.85 / \mathrm{kW}$ & $\$ 12.24 / \mathrm{kW}$ & $\$ 0.00 / \mathrm{kW}$ \\
\hline $2-6$ & $\$ 0.00 / \mathrm{kW}$ & $\$ 3.42 / \mathrm{kW}$ & $\$ 14.35 / \mathrm{kW}$ & $\$ 19.85 / \mathrm{kW}$ & $\$ 12.24 / \mathrm{kW}$ & $\$ 0.00 / \mathrm{kW}$ \\
\hline $4-8$ & $\$ 0.00 / \mathrm{kW}$ & $\$ 3.42 / \mathrm{kW}$ & $\$ 14.35 / \mathrm{kW}$ & $\$ 19.85 / \mathrm{kW}$ & $\$ 12.24 / \mathrm{kW}$ & $\$ 0.00 / \mathrm{kW}$ \\
\hline \multicolumn{7}{|c|}{ Aggregators in Day-Of Option } \\
\hline Product & May & June & July & August & September & October \\
\hline $1-4$ & $\$ 0.00 / \mathrm{kW}$ & $\$ 4.27 / \mathrm{kW}$ & $\$ 17.94 / \mathrm{kW}$ & $\$ 24.81 / \mathrm{kW}$ & $\$ 15.30 / \mathrm{kW}$ & $\$ 0.00 / \mathrm{kW}$ \\
\hline $2-6$ & $\$ 0.00 / \mathrm{kW}$ & $\$ 4.27 / \mathrm{kW}$ & $\$ 17.94 / \mathrm{kW}$ & $\$ 24.81 / \mathrm{kW}$ & $\$ 15.30 / \mathrm{kW}$ & $\$ 0.00 / \mathrm{kW}$ \\
\hline $4-8$ & $\$ 0.00 / \mathrm{kW}$ & $\$ 4.27 / \mathrm{kW}$ & $\$ 17.94 / \mathrm{kW}$ & $\$ 24.81 / \mathrm{kW}$ & $\$ 15.30 / \mathrm{kW}$ & $\$ 0.00 / \mathrm{kW}$ \\
\hline
\end{tabular}

\section{Incentives}

Incentives and penalties will depend on the option that is elected. For directly enrolled customers, incentive will be based on capacity nomination for each month and energy incentives during event days, and will reflect the difference between directly enrolled customer specific energy baseline level and energy consumed during event with respects to nomination.

\section{D.2.3.1 CBP Directly to Facilities}

The following set of use cases concern CBP programs which are enacted directly with participant facilities. The use case diagram can be seen in Figures D9 and D10. 


\section{CBP Program Configuration}

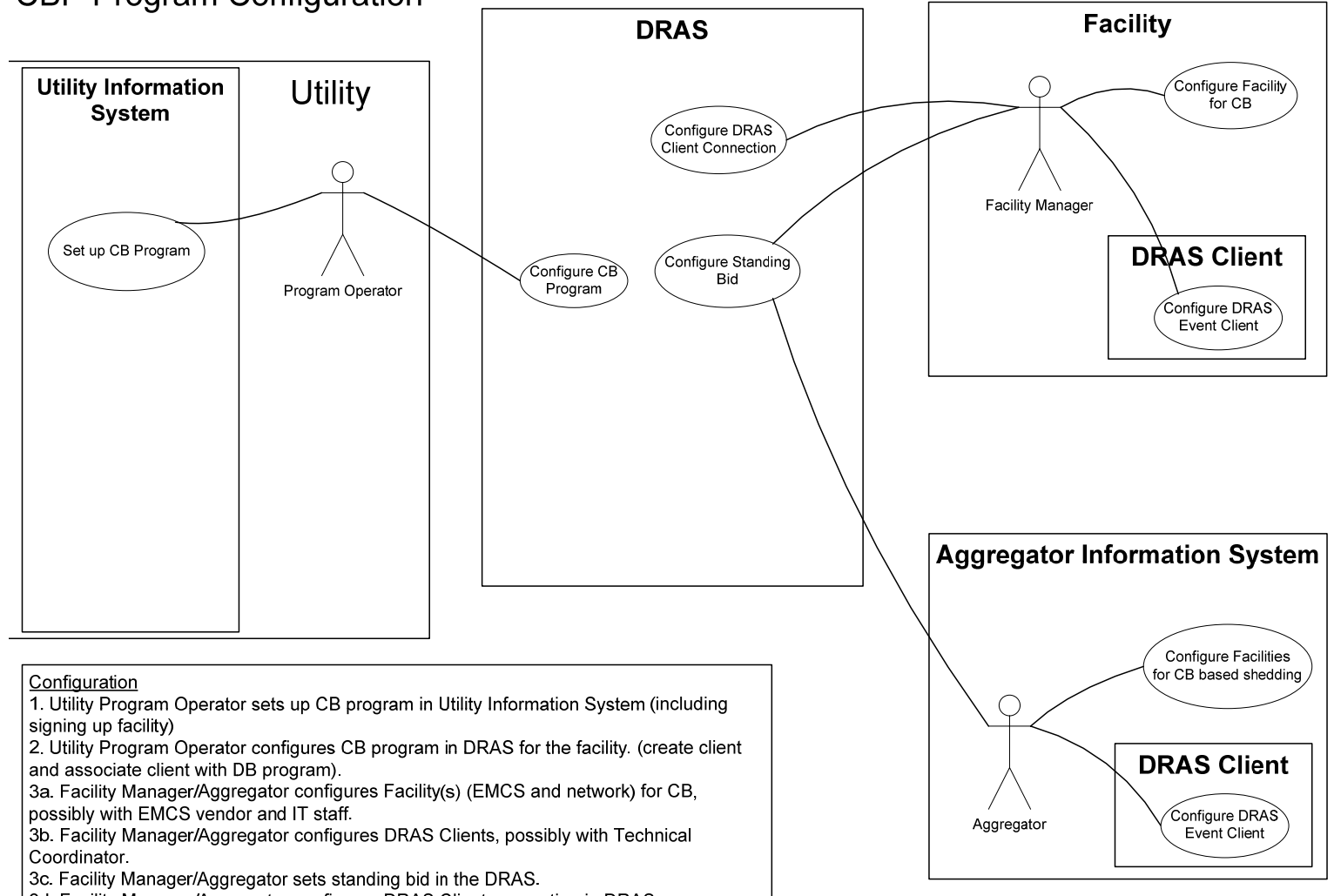

3c. Facility Manager/Aggregator sets standing bid in the DRAs.

3d. Facility Manager/Aggregator configures DRAS Client connection in DRAS.

Figure D9 CBP Program Configuration 


\section{CBP Direct To Facility}

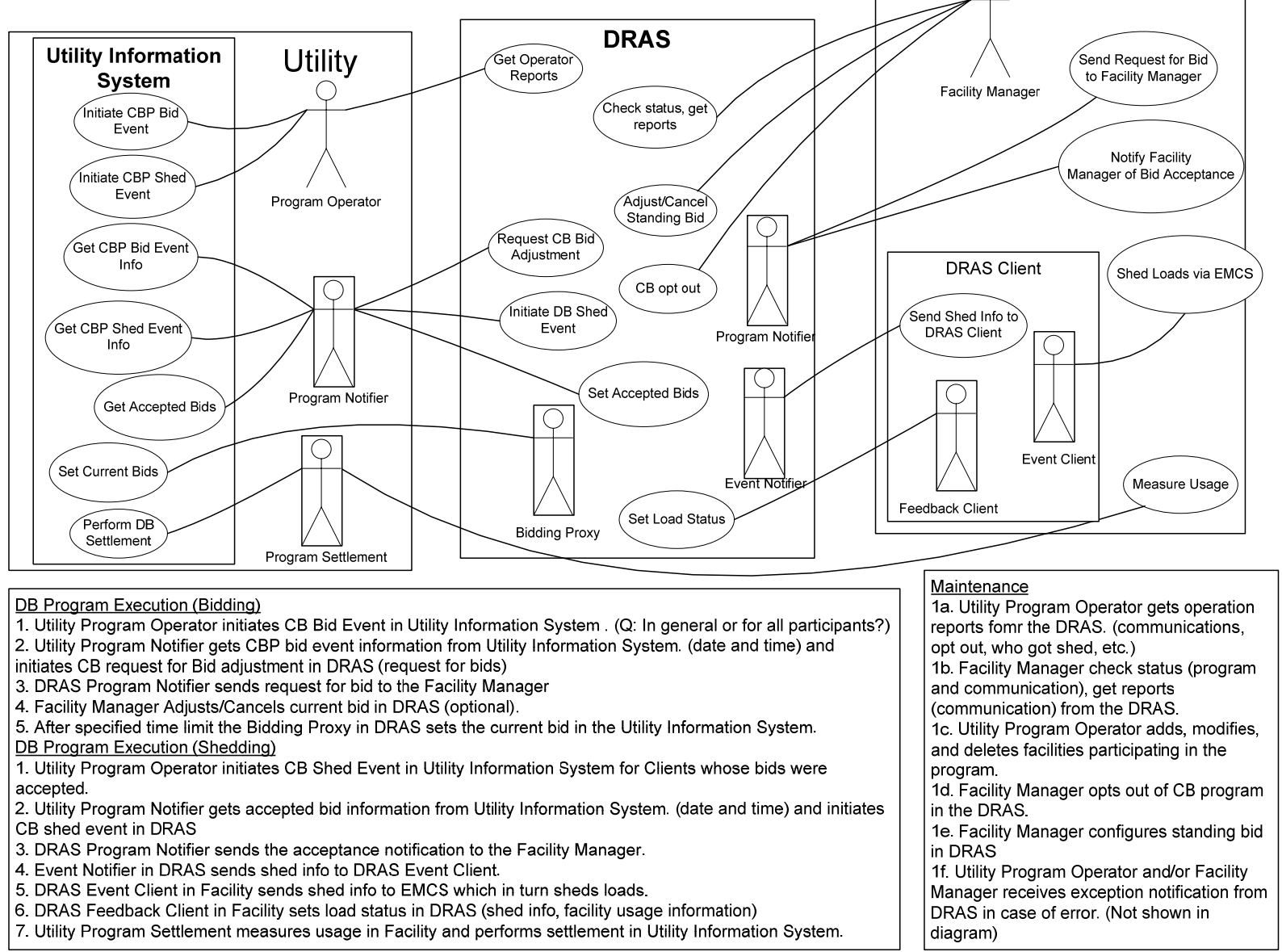

Figure D10. CBP Direct to Facility

\section{D.2.3.1.1 CBP Configuration}

This includes entering all the information necessary for the participant to participate in the DBP DR program and involves the following actions.

1. The utility program operator sets up the DBP program in the Utility Information System. This includes signing up participants and entering all required information necessary for the participant to participate in the DBP program into the UIS. The details of this process are beyond the scope of this document.

2. The utility program operator configures the DBP in the DRAS for the facility. This includes entering information into the DRAS to allow the facility operator to access the DRAS and set up their DRAS Client so that it may communicate with the DRAS. It includes entering the following information:

- Program definition

o Event launching endpoint

o Program to event to DRAS Client signal mapping

- Utility assigned account number used for settlement 
- Customer identification

- Customer password

- Geographic location

- Grid location

3a. The facility manager configures the facility's EMCS and network for DR, possibly in conjunction with the EMCS vendor and IT staff. This could include programming the EMCS to respond to DR events and shed or shift loads appropriately. The details of this process are beyond the scope of this document.

$3 b$. The facility manager configures the DRAS Clients, possibly in conjunction with the technical coordinator. DRAS Clients may take many forms, both in terms of hardware and software. This step configures the DRAS Client so that it can communicate with the Facilities systems that are responsible for managing the loads. The details of this process are beyond the scope of this document.

3c. The facility manager sets the standing bid in the DRAS. This is the bid that will be automatically placed by the DRAS when a request for bids comes from the Utility. It includes the following:

- Load reduction bids per time block (price and load amount)

$3 \mathrm{~d}$. The facility manager configures the DR program parameters and the DRAS Client connection in the DRAS. This step establishes the connection between the DRAS Client and the DRAS. Typically this includes the following types of information:

- Identification and password of the Customer participating in the program

- Contact information, i.e. phone number, pager, email, etc.

- DRAS Client communications parameters

o DRAS IP address

o identification

o password

o IP connection information

o polling frequency if a polling DRAS Client

- Optionally-Load reduction potential (per time block per level)

- Exception parameters

- Opt-out dates

Note that this action is currently depicted as occurring in the DRAS, but depending upon how this step is subsequently implemented in a future OpenADR standards, this may be performed in the DRAS Client and not in the DRAS. 


\section{D.2.3.1.2 CBP Execution}

CBP includes a bidding process, but unlike DBP the request for bids are not linked directly to the shedding events. The bidding process and the shedding process are two distinct sets of steps. Because of this de-coupling it is unlikely that the DRAS would play a useful role in automating the bidding process, but it is still documented here for completeness.

The bidding process includes the following steps:

1. The utility program operator creates the CBP Bidding event in the Utility Information System. In this step a program operator schedules the CBP Bidding event in the Utility Information System. The details of this process are beyond the scope of this document.

2. The utility program notifier gets the CBP bidding event information from the Utility Information System and initiates the CBP Request for bids in the DRAS. The information sent to the DRAS by the utility program notifier sub-system includes the following information:

- Program type

- Date and time of the event

- Date and time issued

- Geographic location

- Customer list (account numbers)

- Request For Bids (RFB) issue date and time

- RFB close time

- Price offered for load reduction per time block

3. The DRAS program notifier sends the request for bid to the facility manager. This notification typically comes in the form of an email, phone call or page.

4. The facility manager adjusts or cancels their current bid in the DRAS. This is an optional step and allows the Manager to adjust their bid for that particular event. If this step is not performed, the DRAS will submit the standing bid after a preset period of time.

5. After the pre-bid time limit has expired, the Bidding Proxy in the DRAS sets the current bid in the Utility Information System. The information sent by the DRAS includes the following:

- Customer account number

- Load reduction bids per time block

The shedding process includes the following steps:

1. The utility program operator creates the CBP Shed event in the Utility Information System. In this step a program operator schedules the CBP Shed 
event in the Utility Information System. The details of this process are beyond the scope of this document.

2. The utility program notifier gets the accepted bids from the Utility Information System and initiates the CB Shed in the DRAS. The accepted bids form the set of participants that the DRAS will send CB shed or shift events to. The information concerning accepted bids includes:

- Customer list (account number)

- Accept or reject

- Load reduction bids per time block (for verification)

3. The DRAS program notifier sends the acceptance notification to the facility manager. The manager is notified of an accepted bid via phone, email, or page.

4. The event notifier in the DRAS sends the CB event information to all the DRAS Clients whose bids were accepted. The DBP event information sent to the DRAS Clients includes the following information:

- Utility event information for Intelligent DRAS Clients

o Date and time of the event

o Date and time issued

o Geographic location (optional)

o Mode and pending signals

- Mode signal levels for Simple DRAS Clients (e.g. normal, moderate, high)

- Event pending signal for Simple DRAS Clients (e.g. yes/no, or simple quantification of how far in advance notice is to be sent)

5. The DRAS Event Client for the facility sends the shed or shift information to the EMCS which in turn causes loads to be shed or shift in related facilities. How this process is done is beyond the scope of this document.

6. The DRAS Feedback Client for the participant facility sends the system load status information to the DRAS. This is a feedback mechanism that is used to record how the facility responded to the DR event. It includes the following information:

- Program identifier

- Facility identifier

- Date and time of the event (shed or shift)

- Shed data in $\mathrm{kW} / \mathrm{kWh}$

- Load reduction end uses (HVAC, lighting, etc.)

- Event Type (Day-Ahead or Day-Of) 
7. The utility program settlement measures usage in the facility and performs settlement in the Utility Information System. How this process is done is beyond the scope of this document.

\section{D.2.3.1.3 CBP Maintenance}

This scenario consists of a set of actions which are necessary to maintain the DBP program. Unlike the Configuration and Execution scenarios this set of actions are less a prescribed set of steps and more a set of possible actions that may be performed by the various roles at unrelated times.

1a. The utility program operator gets the operation reports. The utility program operator can get the following status information from the DRAS at any time:

- Event status (for all participants)

o current outstanding events

- Load reduction potential based upon all customers in program (optional)

- Feedback from DRAS Clients (for those that have optional feedback)

o event logs

- Bids and signal levels per time block for current events (for all participants, indivually and grouped together)

1b. The facility manager checks status. The facility manager can get the following status information from the DRAS at any time:

- DRAS Client communications status

o current status

o last contact

o current signals levels

o current customer manual control levels (opt-out)

o communication logs

o signal logs

o manual control logs

- Event status (same as above)

- Bids and signal levels per time block for current events

1c. The utility program operator adds, modifies or deletes facilities participating in the program. This is similar to the original configuration step.

1d. The facility manager opts out of the DR program. At any time, the facility manager can opt out of the DR program on the DRAS. When there is an opt-out condition, DR events are not propagated to the DRAS Client. The opt-out can be either for the entire program or a single event.

1e. The facility manager adjusts their standing bid in the DRAS. 
1f. The utility program operator and/or the facility manager receive an exception notification from the DRAS in case of an error (this is not shown in the diagram). When an error condition occurs in the DRAS which may require some sort of action by either the participant or the utility the DRAS will send a message to the respective operators via email, voice or pager. Note that this alarming interface does not cover exception conditions that are part of the DRAS operation and managed by the DRAS operator, which is outside the scope of this document. The types of exceptions covered by this interface include:

- DRAS Client communications failure.

\section{D.2.3.2 CBP via Aggregators}

The following set of use cases concern CBP programs which are enacted with aggregators. The use case diagram based on difference configurations can be seen in Figures D10 and D11.

\section{CBP Direct To Facility}

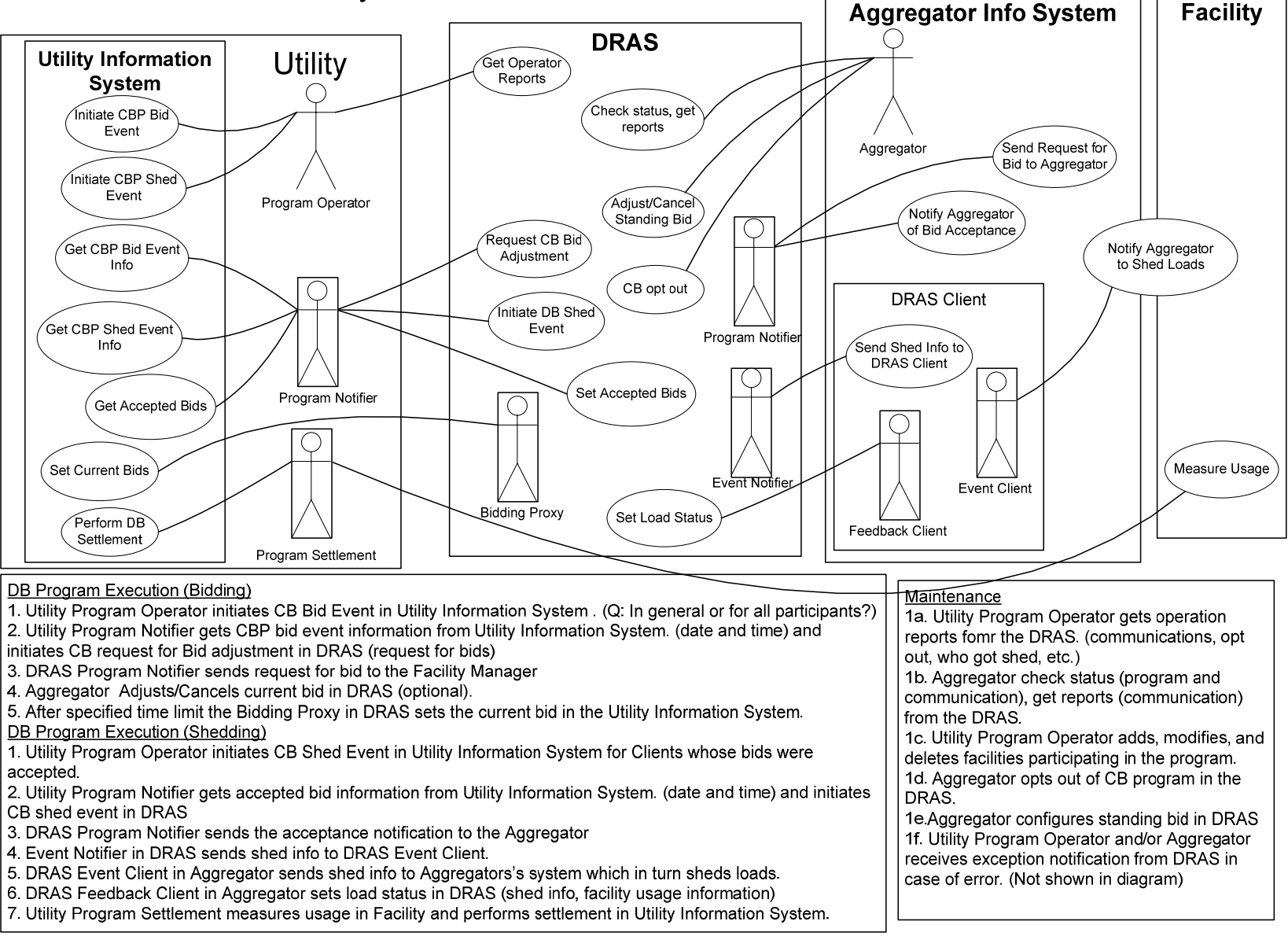

Figure D11. CBP Direct to Facility 


\section{D.2.3.2.1 CBP Configuration}

This includes entering all the information necessary for the participant to participate in the DBP DR program and involves the following actions:

1. The utility program operator sets up the DBP program in the Utility Information System. This includes signing up participants and entering all required information necessary for the participant to participate in the DBP program into the UIS. The details of this process are beyond the scope of this document.

2. The utility program operator configures the DBP in the DRAS for the facility. This includes entering information into the DRAS to allow the facility operator to access the DRAS and set up their DRAS Client so that it may communicate with the DRAS. It includes entering the following information:

- Program definition

o Event launching endpoint

o Program to event to DRAS Client signal mapping

- Utility assigned account number used for settlement

- Customer identification

- Customer password

- Geographic location

- Grid location

3a. The aggregator configures their systems for DR. The details of this process are beyond the scope of this document.

$3 \mathrm{~b}$. The aggregator configures the DRAS Clients, possibly in conjunction with the technical coordinator. DRAS Clients may take many forms, both in terms of hardware and software. This step configures the DRAS Client so that it can communicate with the aggregator systems that are responsible for managing the loads. The details of this process are beyond the scope of this document.

3c. The aggregator sets a standing bid in the DRAS. This is the bid that will be automatically placed by the DRAS when a request for bids comes from the utility. It includes the following:

- Load reduction bids per time block (price and load amount)

3d. The aggregator configures DR program parameters and DRAS Client connection in the DRAS. This step establishes the connection between the DRAS Client and the DRAS. Typically this includes the following types of information:

- Identification and password of the Customer participating in the program

- Contact information, i.e. phone number, pager, email, etc.

- DRAS Client communications parameters

- DRAS IP address

o identification 
0 password

o IP connection information

o polling frequency if a polling DRAS Client

- Optionally - Load reduction potential (per time block per level)

- Exception parameters

- Opt-out dates

Note that this action is currently depicted as occurring in the DRAS, but depending upon how this step is subsequently implemented in a future OpenADR standards, this may be performed in the DRAS Client and not in the DRAS.

\section{D.2.3.2.2 CBP Execution}

CBP includes a bidding process, but unlike DBP the request for bids are not linked directly to the shedding events. Therefore the bidding process and the shedding process are two distinct sets of steps. Because of this de-coupling it is unlikely that the DRAS would play a useful role in automating the bidding process, but it is documented here for completeness.

The bidding process includes the following steps:

1. The utility program operator creates the CBP Bidding event in the Utility Information System. In this step a program operator schedules a CBP Bidding event in the Utility Information System. The details of this process are beyond the scope of this document.

2. The utility program notifier gets the CBP bidding event information from the Utility Information System and initiates the CBP Request for bids in the DRAS. The information sent to the DRAS by the utility program notifier sub-system includes the following information:

- Program type

- Date and time of the event

- Date and time issued

- Geographic location

- Customer list (account numbers)

- Request For Bids (RFB) issue date and time

- RFB close time

- Price offered for load reduction per time block

3. The DRAS program notifier sends the request for bid to the aggregator. This notification typically comes in the form of an email, phone call or page.

4. The aggregator adjusts or cancels the current bid in the DRAS. This is an optional step and allows the Manager to adjust their bid for that particular event. If this 
step is not performed then the DRAS will submit the standing bid after some preset period of time.

5. After the pre-bid time limit has expired, the Bidding Proxy in the DRAS sets the current bid in the Utility Information System. The information sent by the DRAS includes the following:

- Customer account number

- Load reduction bids per time block

The shedding process includes the following steps:

1. The utility program operator creates the CBP Shed event in the Utility Information System. In this step a program operator schedules a CBP Shed event in the Utility Information System. The details of this process are beyond the scope of this document.

2. The utility program notifier gets accepted bids from the Utility Information System and initiates the CBP Shed in the DRAS. The accepted bids form the set of participants that the DRAS will send the CB shed or shift events to. The information concerning accepted bids include:

- Customer list (account number)

- Accept or reject

- Load reduction bids per time block (for verification)

3. The DRAS program notifier sends the acceptance notification to the aggregator. The aggregator operator is notified of an accepted bid via phone, email, or page.

4. The event notifier in the DRAS sends the CBP event information to all DRAS Clients whose bids were accepted. The CBP event information sent to the DRAS Clients includes:

- Utility event information for Intelligent DRAS Clients

o Date and time of the event

o Date and time issued

o Geographic location (optional)

o Mode and pending signals

- Mode signal levels for Simple DRAS Clients (e.g. normal, moderate, high)

- Event pending signal for Simple DRAS Clients (e.g. yes/no, or simple quantification of how far in advance notice is to be sent)

5. The DRAS Event Client for the facility sends shed or shift information to the aggregator's system which in turn causes the aggregator to shed or shift loads in various facilities. How this process is done is beyond the scope of this document.

6. The DRAS Feedback Client in the aggregator's system sends the system load status to the DRAS. This is a feedback mechanism that is used to record how the facility responded to the DR event. It includes the following information: 
- Program identifier

- Facility identifier

- Date and time of the event (shed or shift)

- Shed data in $\mathrm{kW} / \mathrm{kWh}$

- Load reduction end uses (HVAC, lighting, etc.)

- Event Type (Day-Ahead or Day-Of)

7. The utility program settlement measures usage in the facility and performs settlement in the Utility Information System. The details of this process are beyond the scope of this document.

\section{D.2.3.2.3 CBP Maintenance}

This scenario consists of a set of actions which are necessary to maintain the DBP program. Unlike the Configuration and Execution scenarios this set of actions are less a prescribed set of steps and more a set of possible actions that may be performed by the various roles at unrelated times.

1a. The utility program operator gets the operation reports. The utility program operator can get the following status information from the DRAS at any time:

- Event status (for all participants)

o current outstanding events

- Load reduction potential based upon all customers in program (optional)

- Feedback from DRAS Clients (for those that have optional feedback)

o event logs

- Bids and signal levels per time block for current events (for all participants, indivually and grouped together)

$1 \mathrm{~b}$. The aggregator checks status. The aggregator can get the following status information from the DRAS at any time:

- DRAS Client communications status

o current status

o last contact

o current signals levels

o current customer manual control levels (opt-out)

o communication logs

o signal logs

o manual control logs

- Event status (same as above)

- Bids and signal levels per time block for current events 
1c. The utility program operator adds, modifies or deletes facilities participating in the program. This is similar to the original configuration step.

$1 \mathrm{~d}$. The aggregator opts out of the DR program. At any time, the aggregator or the aggregator acting as facility manager can opt out of the DR program on the DRAS. When there is an opt-out condition, DR events are not propagated to the DRAS Client. The opt-out can be either for the entire program or a single event.

1e. The aggregator adjusts their standing bid in the DRAS.

1f. The utility program operator and/or the aggregator receive an exception notification from the DRAS in case of an error (this is not shown in the diagram). When an error condition occurs in the DRAS which may require some sort of action by either the participant or the utility, the DRAS will send a message to the respective operators via email, voice or pager. Note that this alarming interface does not cover exception conditions that are part of the DRAS operation and managed by the DRAS operator, which is outside the scope of this document. The types of exceptions covered by this interface include:

- DRAS Client communications failure.

\section{D.2.4 Base Interruptible Program (BIP)}

The Base Interruptible Program (BIP) is offered by PG\&E. PG\&E's website, http://www.pge.com/mybusiness/energysavingsrebates/demandresponse/baseinterrupti ble/, provides the following description of this program:

The Base Interruptible Program, or E-BIP, is a program that pays to reduce your electricity consumption to a pre-determined amount when the California Independent System Operator (CAISO) issues a load curtailment notice or in the event of a local reliability emergency. There are two separate options for participation. The mandatory option (option A) gives participating customers a monthly payment for committing to reduce electricity demand to a predetermined point when called upon. The voluntary option (option B) pays customers a fixed amount per kilowatt reduced during a curtailment event.

\section{How It Works}

To participate in the program, participants must identify a designated load, or "firm service level", below your average peak demand. To participate, you must set a firm service level at least 15 percent of your highest monthly maximum load with a minimum designated load of $100 \mathrm{~kW}$. New participants must elect one of two available options.

\section{Option A:}

- Gives the customer a 30 minute notification prior to mandatory curtailment of load exceeding the established firm service level. 
- Monthly participation incentives are determined by the Potential Load Reduction as calculated by the difference between the customer's average monthly seasonal demand and the designated firm service level.

- Monthly incentives are determined by the following table:

\begin{tabular}{|c|c|}
\hline Potential Load reduction & Monthly Incentive \\
\hline $500 \mathrm{~kW}$ and below & $\$ 8.00$ per kW \\
\hline $501 \mathrm{~kW}-1,000 \mathrm{~kW}$ & $\$ 8.50$ per kW \\
\hline $1,001 \mathrm{~kW}$ and above & $\$ 9.00$ per kW \\
\hline
\end{tabular}

Failure to reduce loads during an event will result in a $\$ 6.00$ charge per kilowatt hour for energy use over the firm service level.

\section{Option B:}

- Gives the customer a four-hour notification and $\$ 0.60$ per $\mathrm{kWh}$ incentive for a qualifying load reduction during the curtailment event.

- To qualify for the incentive, customers must reduce load to within $15 \%$ of the designated firm service level.

- There is no penalty for failing to reduce loads during an event.

To participate, you must commit to curtail: At least 15 percent of your average monthly load or a minimum of $100 \mathrm{~kW}$, whichever is greater

E-BIP curtailment events are limited to:

Option A: A maximum of one event per day and four hours per event. The program will not exceed 10 events per month, or 120 hours per calendar year.

Option B: There are no event limits on this voluntary option.

\section{Aggregators}

A customer may enroll directly with Pacific Gas and Electric Company or with a third-party aggregator. An aggregator is an entity appointed by a customer, to act on behalf of the customer with respect to all aspects of the program, including receipt of notices, receipt of incentive payments, and payment of penalties.

\section{D.2.4.1 BIP Directly to Facilities}

The following set of use cases concern BIP programs which are enacted directly with participant facilities. The use case diagram can be seen in Figure D12. 


\section{BIP Direct To Facility}

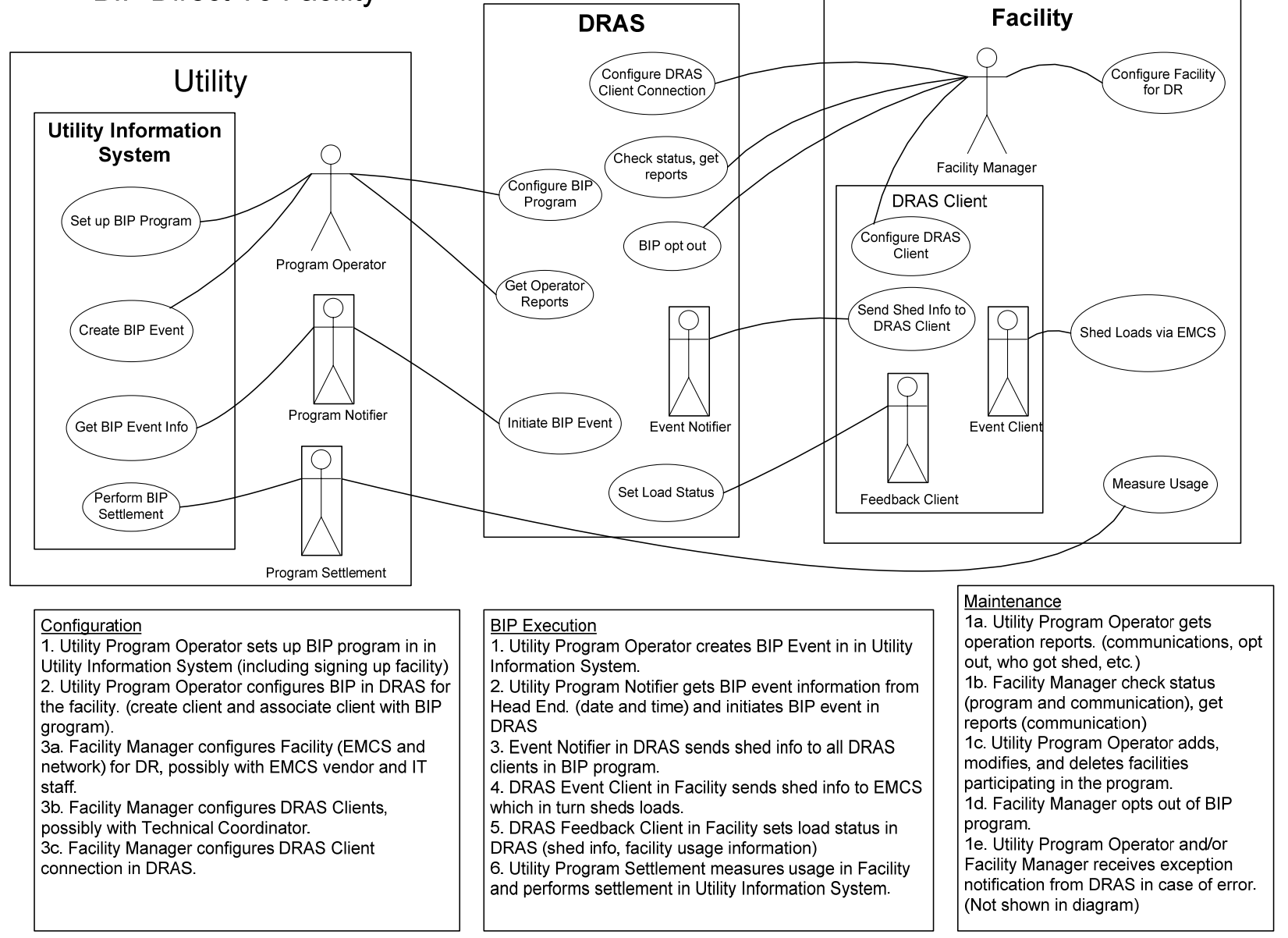

Figure D12. BIP Direct to Facility

\section{D.2.4.1.1 BIP Configuration}

This includes entering all the information necessary for the participant to participate in the BIP DR program and involves the following actions.

1. The utility program operator sets up the BIP program in the Utility Information System. This includes signing up participants and entering all required information necessary for the participant to participate in the BIP program into the UIS. The details of this process are beyond the scope of this document.

2. The utility program operator configures the BIP in the DRAS for the facility. This includes entering information into the DRAS to allow the facility operator to access the DRAS and set up their DRAS Client so that it may communicate with the DRAS. It includes entering the following information:

- Program definition

o Event launching endpoint

o Program to event to DRAS Client signal mapping

- Utility assigned account number used for settlement 
- Customer identification

- Customer password

- Geographic location

- Grid location

3a. The facility manager configures the facility's EMCS and network for DR, possibly in conjunction with the EMCS vendor and IT staff. This could include programming the EMCS to respond to DR events and shed or shift loads appropriately. The details of this process are beyond the scope of this document.

$3 b$. The facility manager configures DRAS Clients, possibly in conjunction with the technical coordinator. DRAS Clients may take many forms, both in terms of hardware and software. This step configures the DRAS Client so that it can communicate with the Facility's systems that are responsible for managing the loads. The details of this process are beyond the scope of this document.

3c. The facility manager configures the DR program parameters and DRAS Client connection in the DRAS. This step establishes the connection between the DRAS Client and the DRAS. Typically this includes the following types of information:

- Identification and password of the Customer participating in the program.

- Contact information, i.e. phone number, pager, email, etc.

- DRAS Client communications parameters

o DRAS IP address

o identification

o password

o IP connection information

o polling frequency if a polling DRAS Client

- Optionally-Load reduction potential (per time block per level)

- Exception parameters

- Opt-out dates

Note that this action is currently depicted as occurring in the DRAS, but depending upon how this step is subsequently implemented in a future OpenADR standards, this may be performed in the DRAS Client and not in the DRAS.

\section{D.2.4.1.2 BIP Execution}

The set of actions needed to execute BIP events include the following steps:

1. The utility program operator creates the BIP event in the Utility Information System. In this step a program operator schedules a BIP event in the Utility Information System. The details of this process are beyond the scope of this document. 
2. The utility program notifier gets the BIP event information from the Utility Information System and initiates the BIP event in the DRAS. The information sent to the DRAS by the utility program notifier sub-system includes the following information:

- Program type

- Date and time of the event

- Date and time issued

- Geographic location

- Customer list (account numbers)

3. The event notifier in the DRAS sends the BIP event information to all DRAS Clients in BIP program. The BIP event information sent to the DRAS Clients includes the following:

- Utility event info for Intelligent DRAS Clients

0 Date and time of the event

o Date and time issued

o Geographic location (optional)

o Mode and pending signals

- Mode signal levels for Simple DRAS Clients (e.g. normal, moderate, high)

- Event pending signal for Simple DRAS Clients (e.g. yes/no, or simple quantification of how far in advance notice is to be sent)

4. The DRAS Event Client for the facility sends shed or shift information to the EMCS which in turn sheds loads. How this process is done is beyond the scope of this document.

5. The DRAS Feedback Client for the facility sends the system load status to the DRAS. This is a feedback mechanism that is used to record how the facility responded to the DR event. It includes the following information:

- Program identifier

- Facility identifier

- Date and time of the event (shed or shift)

- Shed data in $\mathrm{kW} / \mathrm{kWh}$

- Load reduction end uses (HVAC, lighting, etc.)

- Event Type (Day-Ahead or Day-Of)

6. The utility program settlement measures usage in the facility and performs settlement in the Utility Information System. How this process is done is beyond the scope of this document. 


\section{D.2.4.1.3 BIP Maintenance}

This scenario consists of a set of actions which are necessary to maintain the BIP program. Unlike the Configuration and Execution scenarios this set of actions are less a prescribed set of steps and more a set of possible actions that may be performed by the various roles at unrelated times.

1a. The utility program operator gets the operation reports. The utility program operator can get the following status information from the DRAS at any time:

- Event status (for all participants)

o current outstanding events

- Load reduction potential based upon all customers in program (optional)

- Feedback from DRAS Clients (for those that have optional feedback)

o event logs

1b. The facility manager checks the status. The utility program operator can get the following status information from the DRAS at any time:

- DRAS Client communications status

o current status

o last contact

o current signals levels

o current customer manual control levels (opt-out)

o communication logs

o signal logs

o manual control logs

- Event status (same as above)

1c. The utility program operator adds, modifies or deletes facilities participating in the program. This is similar to the original configuration step.

1d. The facility manager opts out of the DR program. At any time, the facility manager can opt out of the DR program on the DRAS. When there is an opt-out condition, DR events are not propagated to the DRAS Client. The opt-out can be either for the entire program or a single event.

1e. The utility program operator and/or the facility manager receive an exception notification from the DRAS in case of an error (this is not shown in the diagram). When an error condition occurs in the DRAS which may require some sort of action by either the participant or the utility the DRAS will send a message to the respective operators via email, voice or pager. Note that this alarming interface does not cover exception conditions that are part of the DRAS operation and managed by the DRAS operator, which is outside the scope of this document. The types of exceptions covered by this interface include: 
- DRAS Client communications failure.

\section{D.2.4.2 BIP via Third Party Aggregators}

The following use cases envision how an aggregator might play a role with the DRAS for BIP. This is depicted in Figure D13. It is very similar to the direct to the facility use case.

BIP via Aggregator

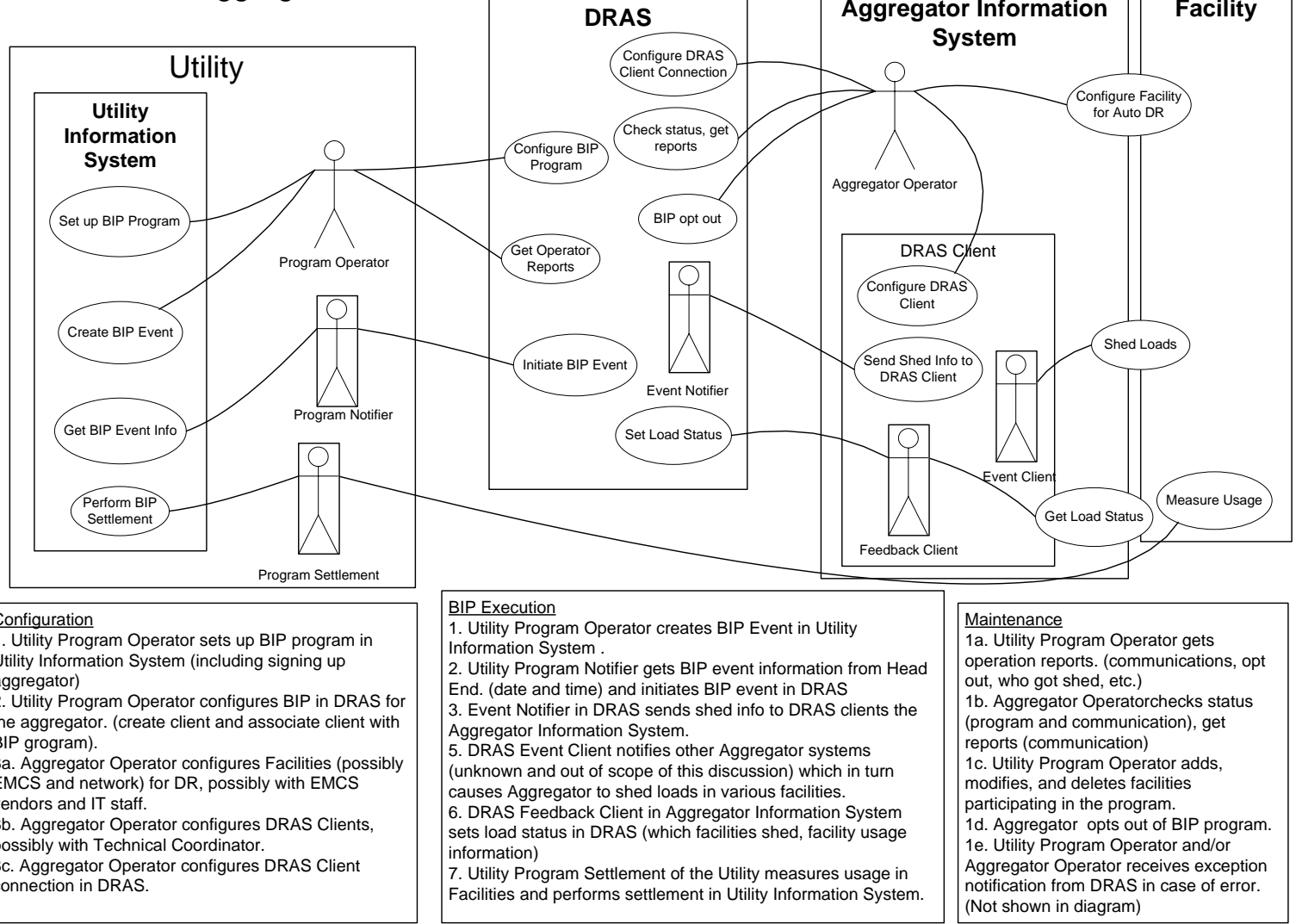

\section{Figure D13. BIP via Aggregator}

\section{D.2.4.2.1 BIP Configuration}

This includes entering all the information necessary for the participant to participate in the BIP DR program and involves the following actions.

1. The utility program operator sets up the BIP program in the Utility Information System. This includes signing up participants and entering all required information necessary for the participant to participate in the BIP program into the UIS. The details of this process are beyond the scope of this document.

2. The utility program operator configures the BIP in the DRAS for the facility. This includes entering information into the DRAS to allow the facility operator to access the DRAS and set up their DRAS Client so that it may communicate with the DRAS. It includes entering the following information:

- Program definition 
o Event launching endpoint

o Program to event to DRAS Client signal mapping

- Utility assigned account number used for settlement

- Customer identification

- Customer password

- Geographic location

- Grid location

3a. The aggregator configures the facility's EMCS and network for DR, possibly in conjunction with the EMCS vendor and IT staff. The details of this process are beyond the scope of this document.

3b. The aggregator configures DRAS Clients, possibly in conjunction with the technical coordinator. DRAS Clients may take many forms, both in terms of hardware and software. This step configures the DRAS Client so that it can communicate with the aggregator's systems that are responsible for managing the loads. The details of this process are beyond the scope of this document.

3c. The aggregator configures the DR program parameters and the DRAS Client connection in the DRAS. This step establishes the connection between the DRAS Client and the DRAS. Typically this includes the following types of information:

- Identification and password of the customer participating in the program

- Contact information, i.e. phone number, pager, email, etc.

- DRAS Client communications parameters

o DRAS IP address

o identification

0 password

o IP connection information

0 polling frequency if a polling DRAS Client

- Optionally-Load reduction potential (per time block per level)

- Exception parameters

- Opt-out dates

Note that this action is currently depicted as occurring in the DRAS, but depending upon how this step is subsequently implemented in a future OpenADR standards, this may be performed in the DRAS Client and not in the DRAS.

\section{D.2.4.2.2 BIP Execution}

The set of actions to execute BIP events include the following steps:

1. The utility program operator creates the BIP event in the Utility Information System. In this step a program operator schedules a BIP event in the Utility 
Information System. The details of this process are beyond the scope of this document.

2. The utility program notifier gets the BIP event information from the Utility Information System and initiates the BIP event in the DRAS. The information sent to the DRAS by the utility program notifier sub-system includes the following:

- Program type

- Date and time of the event

- Date and time issued

- Geographic location

- Customer list (account numbers)

3. The event notifier in the DRAS sends the BIP event information to the appropriate DRAS Clients in BIP program. The BIP event information sent to the DRAS Clients includes the following:

- Utility event information for Intelligent DRAS Clients

o Date and time of the event

o Date and time issued

o Geographic location (optional)

o Mode and pending signals

- Mode signal levels for Simple DRAS Clients (e.g. normal, moderate, high)

- Event pending signal for Simple DRAS Clients (e.g. yes/no, or simple quantification of how far in advance notice is to be sent)

4. The DRAS Event Client for the facility sends shed or shift information to the aggregator's system which in turn causes the aggregator to shed or shift loads within facilities. How this process is done is beyond the scope of this document.

5. The DRAS Feedback Client in the aggregator's system sends the system load status information to the DRAS. This is a feedback mechanism that is used to record how the facility responded to the DR event. It includes the following information:

- Program identifier

- Facility identifier

- Date and time of the event (shed or shift)

- Shed data in $\mathrm{kW} / \mathrm{kWh}$

- Load reduction end uses (HVAC, lighting, etc.)

- Event Type (Day-Ahead or Day-Of) 
6. The utility program settlement measures usage in the facility and performs settlement in the Utility Information System. How this process is done is beyond the scope of this document.

\section{D.2.4.2.3 BIP Maintenance}

This scenario consists of a set of actions which are necessary to maintain the BIP program. Unlike the configuration and execution scenarios this set of actions are less a prescribed set of steps and more a set of possible actions that may be performed by the various roles at unrelated times.

1a. The utility program operator gets the operation reports. The utility program operator can get the following status information from the DRAS at any time:

- Event status (for all participants)

o current outstanding events

- Load reduction potential based upon all customers in program (optional)

- Feedback from DRAS Clients (for those that have optional feedback)

o event logs

1b. The facility manager checks status. The facility manager can get the following status information from the DRAS at any time:

- DRAS Client communications status

o current status

o last contact

o current signals levels

o current customer manual control levels (opt-out)

o communication logs

o signal logs

o manual control logs

- Event status (same as above)

1c. The utility program operator adds, modifies or deletes facilities participating in the program. This is similar to the original configuration step.

1d. The aggregator opts out of the DR program. At any time, the aggregator or the aggregator acting as facility manager can opt out of the DR program on the DRAS. When there is an opt-out condition, DR events are not propagated to the DRAS Client. The opt-out can be either for the entire program or a single event.

1e. The utility program operator and/or the aggregator receive an exception notification from the DRAS in case of an error (this is not shown in the diagram). When an error condition occurs in the DRAS which may require some sort of action by either the participant or the utility the DRAS will send a message to the 
respective operators via email, voice or pager. Note that this alarming interface does not cover exception conditions that are part of the DRAS operation and managed by the DRAS operator, which is outside the scope of this document. The types of exceptions covered by this interface include:

- DRAS Client communications failure.

\section{D.2.5 Peak Day Credit (PDC)}

Peak Day Credit (PDC) is a DR program offered by San Diego Gas \& Electric (SDG\&E). The following is a brief description from the SDG\&E web site, http://www.sdge.com/peakday2020/:

What is the Peak Day Credit program?

Participating businesses can receive a credit on their bill when they reduce their electric usage an average of 10-20\% during on-peak hours when given one-day advance notice. Peak Day is part of SDG\&E's Demand Response program portfolio-a component of SDG\&E's long-term resource plan, and one of the ways SDG\&E is helping the regional economy.

\section{How does the program work?}

You will receive notification the day before a peak energy day is called. On a peak energy day, you will be asked to reduce your electricity consumption by an average of $10-20 \%$ from 11 a.m. to 6 p.m. The program runs through September 30.

\section{How is a peak energy day determined?}

A peak energy day may be called one of two ways: 1) when both the next day forecasted temperature at Miramar Marine Corps Air Station is 84 degrees or higher, and the current day's local electric system load reaches 3,620 megawatts; 2) due to extreme conditions such as an alert called by the California Independent System Operator.

\section{D.2.5.1 PDC Directly to Facilities}

The following set of use cases concern PDC programs which are enacted directly with participant facilities. The use case diagram can be seen in Figure D14. 
PDC Direct To Facility

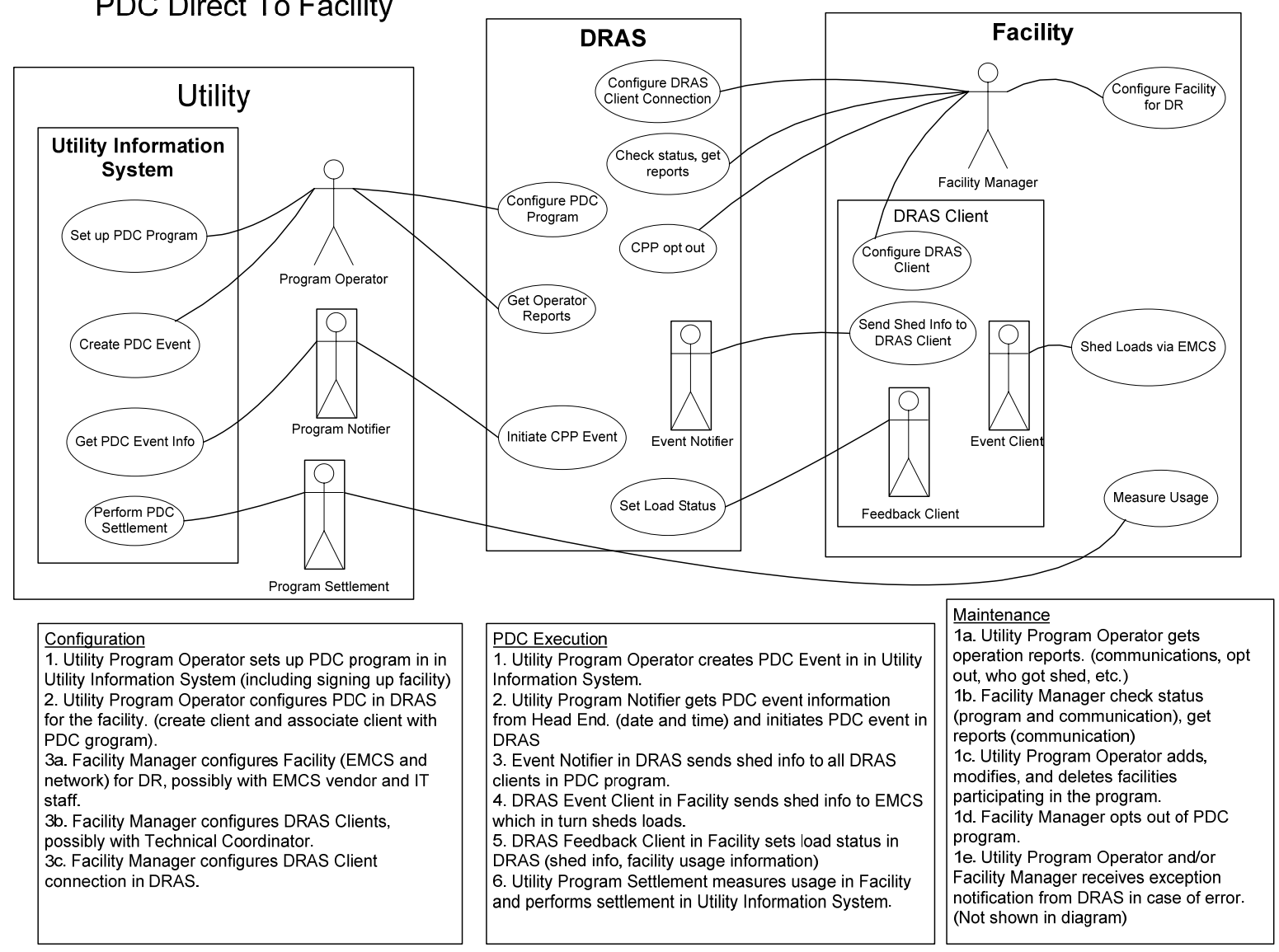

Figure D14. PDC Direct to Facility

\section{D.2.5.1.1 PDC Configuration}

This includes entering all the information necessary for the participant to participate in the PDC DR program and involves the following actions.

1. The utility program operator sets up the PDC program in the Information System. This includes signing up participants and entering all required information necessary for the participant to participate in the PDC program into the UIS. The details of this process are beyond the scope of this document.

2. The utility program operator configures the PDC in the DRAS for the facility. This includes entering information into the DRAS to allow the facility operator to access the DRAS and set up their DRAS Client so that it may communicate with the DRAS. It includes entering the following information:

- Program definition

o Event launching endpoint

o Program to event to DRAS Client signal mapping

- Utility assigned account number used for settlement

- Customer identification 
- Customer password

- Geographic location

- Grid location

3a. The facility manager configures the facility's EMCS and network for DR, possibly in conjunction with the EMCS vendor and IT staff. This could include programming the EMCS to respond to DR events and shed or shift loads appropriately. The details of this process are beyond the scope of this document.

$3 b$. The facility manager configures the DRAS Clients, possibly in conjunction with the technical coordinator. DRAS Clients may take many forms, both in terms of hardware and software. This step configures the DRAS Client so that it can communicate with the Facilities systems that are responsible for managing the loads. The details of this process are beyond the scope of this document.

3c. The facility manager configures the DR program parameters and DRAS Client connection in the DRAS. This step establishes the connection between the DRAS Client and the DRAS. Typically this includes the following types of information:

- Identification and password of the Customer participating in the program.

- Contact information, i.e. phone number, pager, email, etc.

- DRAS Client communications parameters

o DRAS IP address

o identification

o password

o IP connection information

o polling frequency if a polling DRAS Client

- Optionally - Load reduction potential (per time block per level)

- Exception parameters

- Opt-out dates

Note that this action is currently depicted as occurring in the DRAS, but depending on how this step is subsequently implemented in a future OpenADR standards, this may be performed in the DRAS Client and not in the DRAS.

\section{D.2.5.1.2 PDC Execution}

The set of actions to execute PDC events include the following steps:

1. The utility program operator creates the PDC event in the Utility Information System. In this step a program operator schedules a PDC event in the Utility Information System. The details of this process are beyond the scope of this document.

2. The utility program notifier gets the PDC event information from the Utility Information System and initiates PDC event in the DRAS. The information sent 
to the DRAS by the utility program notifier sub-system includes the following information:

- Program type

- Date and time of the event

- Date and time issued

- Geographic location

- Customer list (account numbers)

3. The event notifier in the DRAS sends the PDC event information to the appropriate DRAS Clients in PDC program. The PDC event information sent to the DRAS Clients includes the following:

- Utility event information for Intelligent DRAS Clients

o Date and time of the event

o Date and time issued

o Geographic location (optional)

o Mode and pending signals

- Mode signal levels for Simple DRAS Clients (e.g. normal, moderate, high)

- Event pending signal for Simple DRAS Clients (e.g. yes/no, or simple quantification of how far in advance notice is to be sent)

4. The DRAS Event Client for the facility sends shed or shift information to the EMCS which in turn sheds loads. How this process is done is beyond the scope of this document.

5. The DRAS Feedback Client for the facility sends the system load status to the DRAS. This is a feedback mechanism that is used to record how the Facility responded to the DR event. It includes the following information:

- Program identifier

- Facility identifier

- Date and time of the event (shed or shift)

- Shed data in $\mathrm{kW} / \mathrm{kWh}$

- Load reduction end uses (HVAC, lighting, etc.)

- Event Type (Day-Ahead or Day-Of)

6. The utility program settlement measures usage in Facility and performs settlement in the Utility Information System. How this process is done is beyond the scope of this document.

\section{D.2.5.1.3 PDC Maintenance}

This scenario consists of a set of actions which are necessary to maintain the PDC program. Unlike the configuration and execution scenarios this set of actions are less a 
prescribed set of steps and more a set of possible actions that may be performed by the various roles at unrelated times.

1a. The utility program operator gets the operation reports. The utility program operator can get the following status information from the DRAS at any time:

- Event status (for all participants)

o current outstanding events

- Load reduction potential based upon all customers in program (optional)

- Feedback from DRAS Clients (for those that have optional feedback)

o event logs

1b. The facility manager checks status. The facility manager can get the following status information from the DRAS at any time:

- DRAS Client communications status

o current status

o last contact

o current signals levels

o current customer manual control levels (opt-out)

o communication logs

o signal logs

o manual control logs

- Event status (same as above)

1c. The utility program operator adds, modifies and deletes facilities participating in the program. This is similar to the original configuration step.

1d. The facility manager opts out of the DR program. At any time, the facility manager can opt out of the DR program on the DRAS. When there is an opt-out condition, DR events are not propagated to the DRAS Client. The opt-out can be either for the entire program or a single event.

1e. The utility program operator and/or the facility manager receive an exception notification from the DRAS in case of an error (this is not shown in the diagram). When an error condition occurs in the DRAS which may require some sort of action by either the participant or the utility the DRAS will send a message to the respective operators via email, voice or pager. Note that this alarming interface does not cover exception conditions that are part of the DRAS operation and managed by the DRAS operator, which is outside the scope of this document. The types of exceptions covered by this interface include:

- DRAS Client communications failure. 


\section{D.2.5.2 PDC via Third Party Aggregators}

The following use cases envision how an aggregator might play a role with the DRAS for PDC. This is depicted in Figure D15. It is very similar to the direct to facility use case.

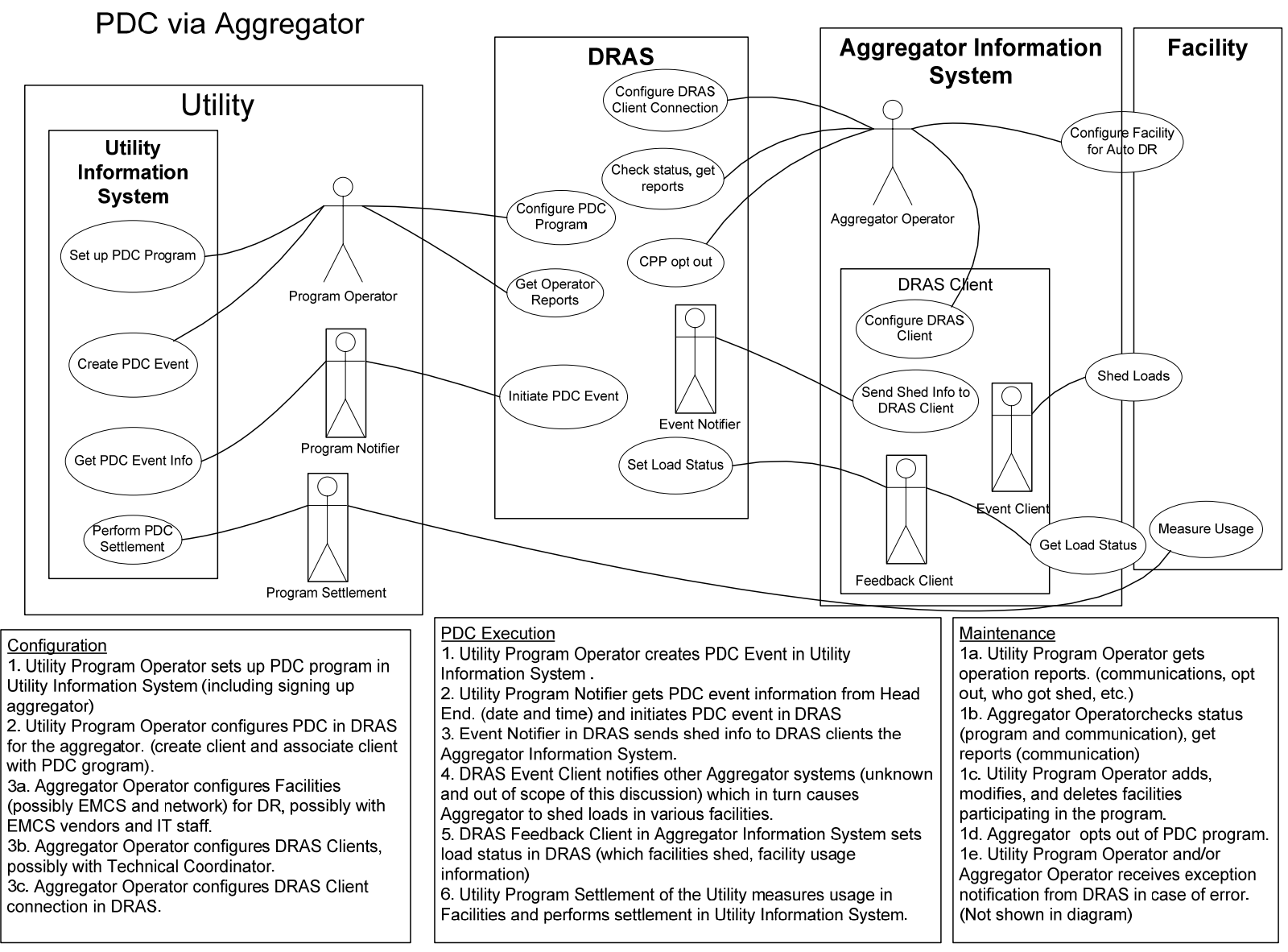

Figure D15. PDC via Aggregator

\section{D.2.5.2.1 PDC Configuration}

This includes entering all the information necessary for the participant to participate in the PDC DR program and involves the following actions.

1. The utility program operator sets up the PDC program in the Utility Information System. This includes signing up participants and entering all required information necessary for the participant to participate in the PDC program into the UIS. The details of this process are beyond the scope of this document.

2. The utility program operator configures the PDC in the DRAS for the facility. This includes entering information into the DRAS to allow the facility operator to access the DRAS and set up their DRAS Client so that it may communicate with the DRAS. It includes entering the following information:

- Program definition 
o Event launching endpoint

o Program to event to DRAS Client signal mapping

- Utility assigned account number used for settlement

- Customer identification

- Customer password

- Geographic location

- Grid location

3a. The aggregator configures the facility's EMCS and network for DR, possibly in conjunction with the EMCS vendor and IT staff. The details of this process are beyond the scope of this document.

$3 \mathrm{~b}$. The aggregator configures the DRAS Clients, possibly in conjunction with the technical coordinator. DRAS Clients may take many forms, both in terms of hardware and software. This step configures the DRAS Client so that it can communicate with the aggregator's systems that are responsible for managing the loads. The details of this process are beyond the scope of this document.

3c. The aggregator configures DR program parameters and DRAS Client connection in the DRAS. This step establishes the connection between the DRAS Client and the DRAS. Typically this includes the following types of information:

- Identification and password of the Customer participating in the program.

- Contact information, i.e. phone number, pager, email, etc.

- DRAS Client communications parameters.

o DRAS IP address

o identification

0 password

o IP connection information

o polling frequency if a polling DRAS Client.

- Optionally - Load reduction potential (per time block per level)

- Exception parameters.

- Opt-out dates

Note that this action is currently depicted as occurring in the DRAS, but depending upon how this step is subsequently implemented in a future OpenADR standards, this may be performed in the DRAS Client and not in the DRAS.

\section{D.2.5.2.2 PDC Execution}

The set of actions to execute PDC events include the following steps:

1. The utility program operator creates the PDC event in the Utility Information System. In this step a program operator schedules the PDC event in the Utility 
Information System. The details of this process are beyond the scope of this document.

2. The utility program notifier gets the PDC event information from the Utility Information System and initiates the PDC event in the DRAS. The information sent to the DRAS by the utility program notifier sub-system includes the following information:

- Program type

- Date and time of the event

- Date and time issued

- Geographic location

- Customer list (account numbers)

3. The event notifier in the DRAS sends PDC event information to all DRAS Clients in PDC program. The PDC event information sent to the DRAS Clients includes the following:

- Utility event information for Intelligent DRAS Clients

o Date and time of the event

o Date and time issued

o Geographic location (optional)

o Mode and pending signals

- Mode signal levels for Simple DRAS Clients (e.g. normal, moderate, high)

- Event pending signal for Simple DRAS Clients (e.g. yes/no, or simple quantification of how far in advance notice is to be sent)

4. The DRAS Event Client in the facility sends shed or shift information to aggregator's system which in turn causes aggregator to shed or shift loads in various facilities. How this process is done is beyond the scope of this document.

5. The DRAS Feedback Client in the aggregator's system sends the system load status to the DRAS. This is a feedback mechanism that is used to record how the facility responded to the DR event. It includes the following information:

- Program identifier

- Facility identifier

- Date and time of the event (shed or shift)

- Shed data in $\mathrm{kW} / \mathrm{kWh}$

- Load reduction end uses (HVAC, lighting, etc.)

- Event Type (Day-Ahead or Day-Of)

6. The utility program settlement measures usage in the facility and performs settlement in the Utility Information System. How this process is done is beyond the scope of this document. 


\section{D.2.5.2.3 PDC Maintenance}

This scenario consists of a set of actions which are necessary to maintain the PDC program. Unlike the configuration and execution scenarios this set of actions are less a prescribed set of steps and more a set of possible actions that may be performed by the various roles at unrelated times.

1a. The utility program operator gets the operation reports. The utility program operator can get the following status information from the DRAS at any time:

- Event status (for all participants)

o current outstanding events

- Load reduction potential based upon all customers in program (optional)

- Feedback from DRAS Clients (for those that have optional feedback)

o event logs

1b. The facility manager checks status. The utility program operator can get the following status information from the DRAS at any time:

- DRAS Client communications status

o current status

o last contact

o current signals levels

o current customer manual control levels (opt-out)

o communication logs

o signal logs

o manual control logs

- Event status (same as above)

1c. The utility program operator adds, modifies or deletes facilities participating in the program. This is similar to the original configuration step.

$1 \mathrm{~d}$. The aggregator opts out of the DR program. At any time, the aggregator or the aggregator acting as facility manager can opt out of the DR program on the DRAS. When there is an opt-out condition, DR events are not propagated to the DRAS Client. The opt-out can be either for the entire program or a single event.

1e. The utility program operator and/or the aggregator receive an exception notification from the DRAS in case of an error (this is not shown in the diagram). When an error condition occurs in the DRAS which may require some sort of action by either the participant or the utility the DRAS will send a message to the respective operators via email, voice or pager. Note that this alarming interface does not cover exception conditions that are part of the DRAS operation and managed by the DRAS operator, which is outside the scope of this document. The types of exceptions covered by this interface include: 
- DRAS Client communications failure.

\section{D.2.6 DR Programs with Programmable Communicating Thermostat (PCT)}

Programmable Communicating Thermostats (PCTs) are currently being developed that in the future may allow small commercial and residential facilities to participate in DR programs. One of the PCT communication modes currently being developed uses a broadcast wireless network such as sub-carrier FM. Such a network would allow large numbers of PCTs to simultaneously receive a single broadcast transmission of information. Upon receiving the information, the PCT can take appropriate action by changing the set point of the HVAC unit that it is controlling, thus providing a means to shed or shift loads.

The use case for the PCT scenario includes a PCT Network Operating Center (NOC) that is responsible for managing all broadcast transmissions to the PCT. The DRAS will interface to the NOC to facilitate communications with the PCT much like it does in the previous use cases. From the DRAS point of view, the PCTs do not represent a radical departure from other participant sites or devices.

This section presents a proposed architecture and use case for communicating with PCTs in DR programs. The use case diagram is depicted in Figure D16.

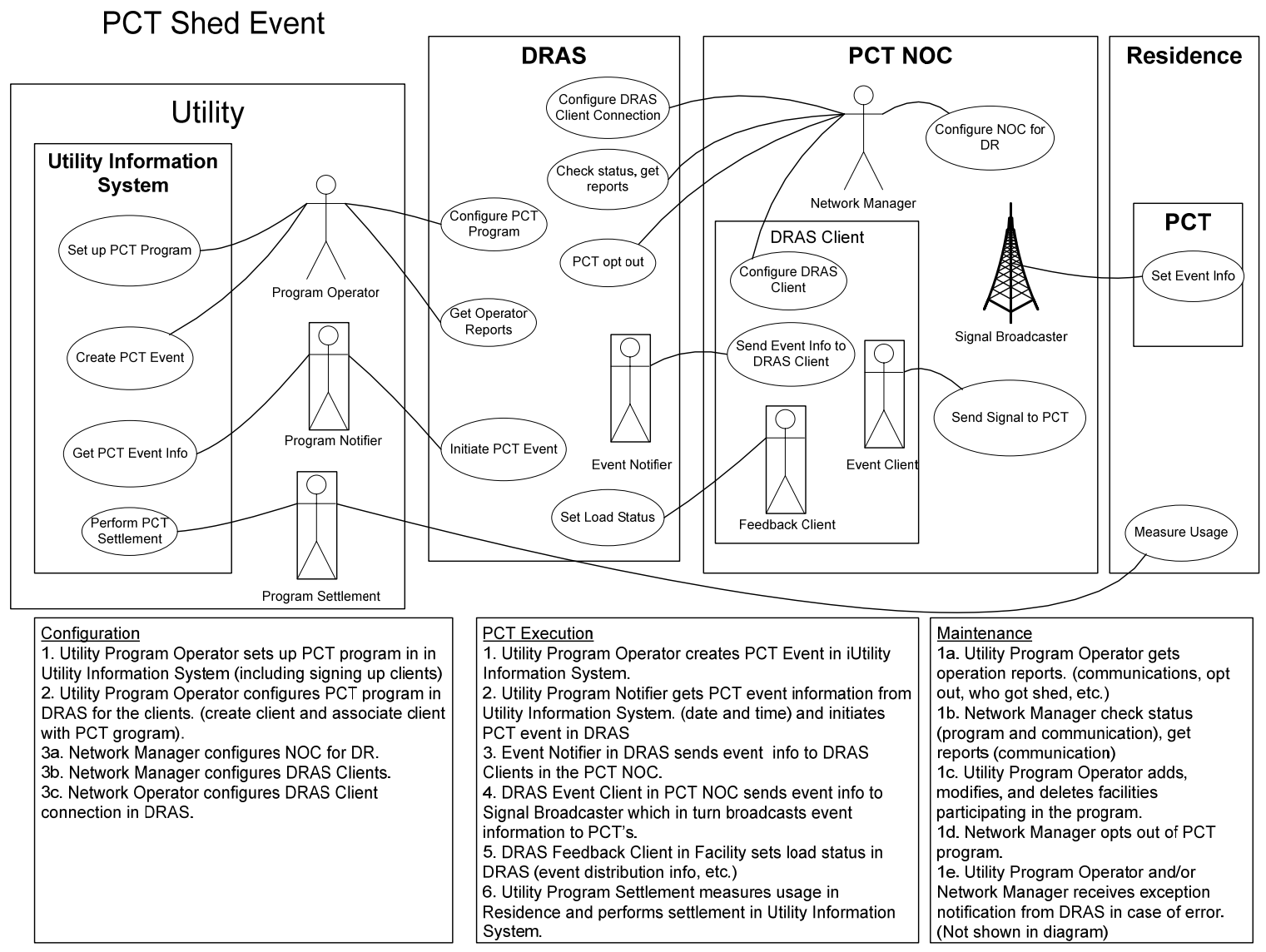

Figure D16. PCT Shed Event 


\section{D.2.6.1.1 PCT DR Configuration}

This includes entering all the information necessary for the participant to participate in the PCT DR program and involves the following actions:

1. The utility program operator sets up the PCT program in the Utility Information System. This includes signing up participants and entering all required information necessary for the participant to participate in the PCT program into the UIS. The details of this process are beyond the scope of this document.

2. The utility program operator configures the PCT DR in the DRAS for the facility. This includes entering information into the DRAS to allow the facility operator to access the DRAS and set up their DRAS Client so that it may communicate with the DRAS. It includes entering the following information:

- Program definition

o Event launching endpoint

o Program to event to DRAS Client signal mapping

- Utility assigned account number used for settlement

- Customer identification

- Customer password

- Geographic location

- Grid location

- Grouping parameter

3a. The Network Manager configures the NOC for the DR program. This may include grouping PCTs to match the grouping parameters set up by the utility DR program. The details of this process are beyond the scope of this document.

3b. The Network Manager configures the DRAS Clients, possibly in conjunction with the technical coordinator. DRAS Clients may take many forms, both in terms of hardware and software. This step configures the DRAS Client so that it can communicate with the Facilities' systems that are responsible for managing the loads. The details of this process are beyond the scope of this document.

3c. The Network Manager configures the DR program parameters and the DRAS Client connection in the DRAS. This step establishes the connection between the DRAS Client and the DRAS. Typically this includes the following types of information:

- Identification and password of the Customer participating in the program

- Contact information, i.e. phone number, pager, email, etc.

- DRAS Client communications parameters

- DRAS IP address

o identification

o password 
o IP connection information

0 polling frequency if a polling DRAS Client

- Optionally-Load reduction potential (per time block per level)

- Exception parameters

- Opt-out dates

Note that this action is currently depicted as occurring in the DRAS, but depending upon how this step is subsequently implemented in a future OpenADR standards, this may be performed in the DRAS Client and not in the DRAS.

\section{D.2.6.1.2 PCT DR Execution}

The set of actions to execute PCT events include the following steps:

1. The utility program operator creates the PCT DR event in the Utility Information System. In this step a program operator schedules a PCT event in the Utility Information System. The details of this process are beyond the scope of this document.

2. The utility program notifier gets the PCT DR event information from the Utility Information System and initiates the PCT DR event in the DRAS. The information sent to the DRAS by the utility program notifier sub-system includes the following:

- Program type

- Date and time of the event

- Date and time issued

- Geographic location

- Customer list (account numbers)

3. The event notifier in the DRAS sends the PCT DR event information to the appropriate DRAS Clients in the PCT DR program. The PCT event information sent to the DRAS Clients includes the following:

- Utility event information for Intelligent DRAS Clients

o Date and time of the event

o Date and time issued

o Geographic location (optional)

o Mode and pending signals

- Mode signal levels for Simple DRAS Clients (e.g. normal, moderate, high)

- Event pending signal for Simple DRAS Clients (e.g. yes/no, or simple quantification of how far in advance notice is to be sent)

4. The DRAS Event Client in the NOC sends event information to the PCT Signal Broadcaster which in turn broadcasts the event information to the PCTs. How this process is done is beyond the scope of this document. 
5. The DRAS Feedback Client in the NOC sends the system load status to the DRAS. This is a feedback mechanism that is used to record how the facility responded to the DR event. It includes the following information:

- Program identifier

- Facility identifier

- Date and time of the event (shed or shift)

- Shed data in $\mathrm{kW} / \mathrm{kWh}$

- Load reduction end uses (HVAC, lighting, etc.)

- Event Type (Day-Ahead or Day-Of)

6. The utility program settlement measures usage in the facility and performs settlement in the Utility Information System. How this process is done is beyond the scope of this document.

\section{D.2.6.1.3 PCT DR Maintenance}

This scenario consists of a set of actions which are necessary to maintain the PCT program. Unlike the configuration and execution scenarios this set of actions are less a prescribed set of steps and more a set of possible actions that may be performed by the various roles at unrelated times.

1a. The utility program operator gets the operation reports. The utility program operator can get the following status information from the DRAS at any time:

- Event status (for all participants)

o current outstanding events

- Load reduction potential based upon all customers in program (optional)

- Feedback from DRAS Clients (for those that have optional feedback)

o event logs

1b. The Network Manager checks status. The utility program operator can get the following status information from the DRAS at any time:

- DRAS Client communications status

o current status

o last contact

o current signals levels

o current customer manual control levels (opt-out)

o communication logs

o signal logs

o manual control logs

- Event status (same as above) 
1c. The utility program operator adds, modifies or deletes facilities participating in the program. This is similar to the original configuration step.

1d. The Network Manager opts out of the DR program. At any time, the Network Manager can opt out of the DR program on the DRAS. When there is an opt-out condition, DR events are not propagated to the DRAS Client. The opt-out can be either for the entire program or a single event.

1e. The utility program operator and/or Network Manager receive an exception notification from the DRAS in case of an error (this is not shown in the diagram). When an error condition occurs in the DRAS which may require some sort of action by either the participant or the utility the DRAS will send a message to the respective operators via email, voice or pager. Note that this alarming interface does not cover exception conditions that are part of the DRAS operation and managed by the DRAS operator, which is outside the scope of this document. The types of exceptions covered by this interface include:

- DRAS Client communications failure.

\section{D.2.7 Generic Real-Time Pricing Based Programs (RTP)}

So far the use cases presented are event based meaning that a DR event is generated that is propagated to the participants. Another useful stream of information that may be used to manage DR programs is Real-Time Pricing (RTP). RTP is somewhat different than an event in that it is potentially a continuously changing quantity that may change more frequently than the normal DR events. This may be a continuous tariff and not a utility program per se. Furthermore there are two modes of operation that may effect how RTP information is used to manage participant loads:

1. Real-time prices are propagated from the Utilities to the participants via the DRAS and the participants then use this information to shed or shift loads.

2. Real-time prices are sent by the Utilities to the DRAS which then in turn generates DR events based upon some configured business logic for a particular participant or program. Since simple DR events are presumably easier for the participants to deal with in their shedding logic this alleviates the need for the participants to do any complicated programming to use the RTP information.

Clearly any use case that uses RTP information should support both the scenarios listed above.

The following use cases show how Real-Time Pricing may be used for DR programs in conjunction with the DRAS. The use case diagram for this scenario is shown in Figure D17. 


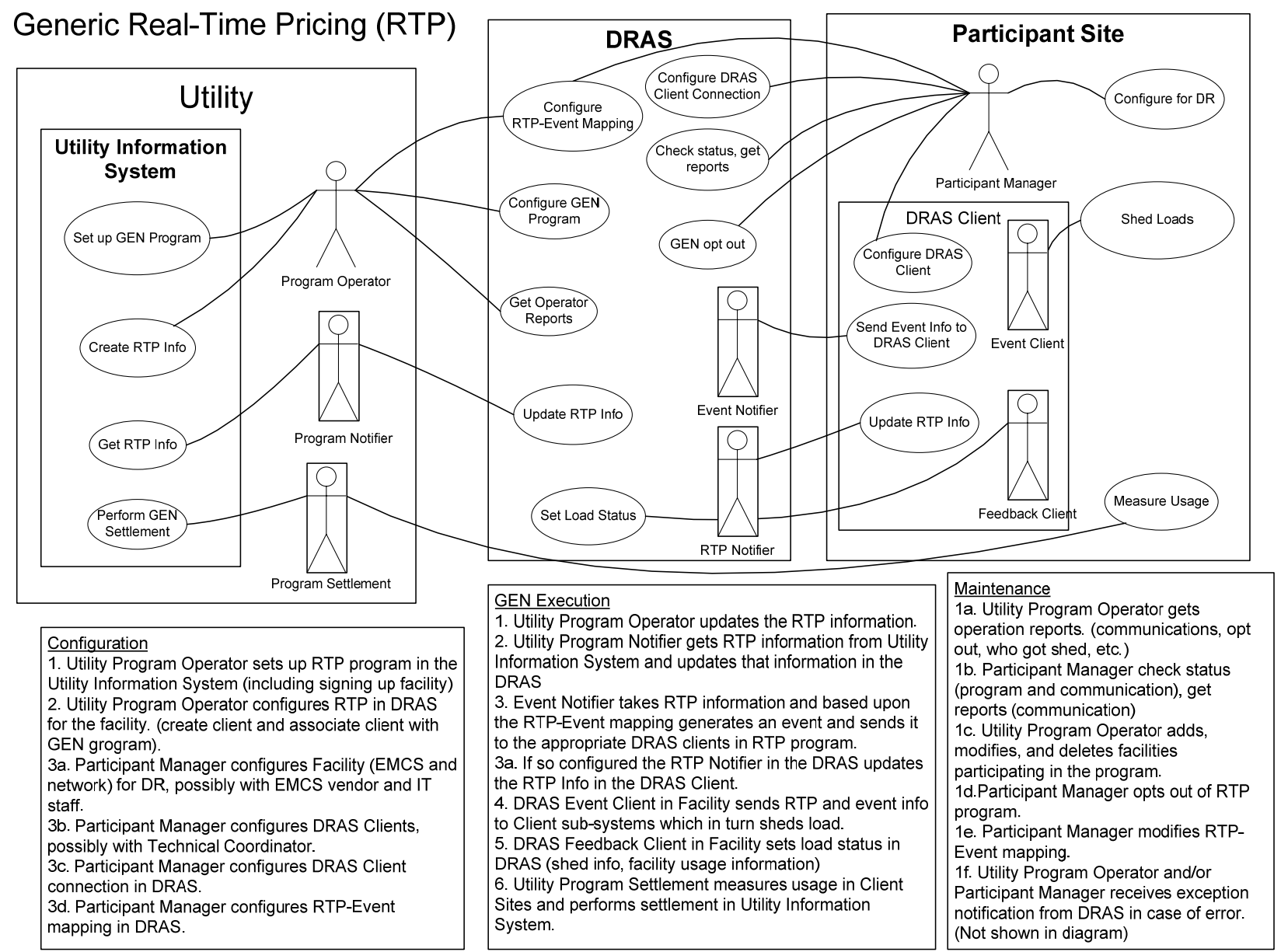

Figure D17. Generic Real-Time Pricing (RTP)

\section{D.2.7.1 RTP Configuration}

This includes entering all the information necessary for the participant to participate in the RTP DR program and involves the following actions:

1. The utility program operator sets up the RTP program in the Utility Information System. This includes signing up participants and entering all required information necessary for the participant to participate in the RTP program into the UIS. The details of this process are beyond the scope of this document.

2. The utility program operator configures the RTP in the DRAS for the facility. This includes entering information into the DRAS to allow the participant Manager to access the DRAS and set up their DRAS Client so that it may communicate with the DRAS. It includes entering the following information:

- Program definition

o Event launching endpoint

o Program to event to DRAS Client signal mapping

- Utility assigned account number used for settlement 
- Customer identification

- Customer password

- Geographic location

- Grid location

3a. The participant manager configures the participant site's EMCS and/or network for DR, possibly in conjunction with the EMCS vendor and IT staff. This could include programming the EMCS to respond to DR events and shed or shift loads appropriately. The details of this process are beyond the scope of this document.

$3 b$. The participant manager configures the DRAS Clients, possibly in conjunction with the technical coordinator. DRAS Clients may take many forms, both in terms of hardware and software. This step configures the DRAS Client so that it can communicate with the Facility's systems that are responsible for managing the loads. The details of this process are beyond the scope of this document.

3c. The participant manager configures the DR program parameters and DRAS Client connection in the DRAS. This step establishes the connection between the DRAS Client and the DRAS. Typically this includes the following types of information:

- Identification and password of the Customer participating in the program

- Contact information, i.e. phone number, pager, email, etc.

- DRAS Client communications parameters

o DRAS IP address

o identification

o password

o IP connection information

o polling frequency if a polling DRAS Client

- Optionally - Load reduction potential (per time block per level)

- Exception parameters

- Opt-out dates

Note that this action is currently depicted as occurring in the DRAS, but depending upon how this step is subsequently implemented in a future OpenADR standards, this may be performed in the DRAS Client and not in the DRAS.

$3 \mathrm{~d}$. The participant manager configures the RTP-event mapping in the DRAS. This is the logic that determines what types of events are generated in response to the RTP information received by the DRAS. Note that the participant site may not receive events at all, but may prefer to receive the RTP information. This is part of the configuration process. Also note that this configuration process may be performed by the program operator of the utility. 


\section{D.2.7.2 RTP Execution}

The set of actions to execute RTP programs include the following steps:

1. The utility program operator updates the RTP information in the Utility Information System. This may be done automatically and could be scheduled to occur at some regular time interval or as pricing information changes and needs to be propagated to the participant as part of the DR program. The details of how the pricing information is changed are beyond the scope of this document.

2. The utility program notifier gets the RTP information from the Utility Information System and updates the pricing information in the DRAS. The information sent to the DRAS by the utility program notifier sub-system includes the following information:

- Program type

- RTP information

- Date and time of the RTP information

- Date and time issued

- Geographic location

- Customer list (account numbers)

3a. The event notifier in the DRAS takes the RTP information and, based upon the RTP-event mapping, generates an event and sends it to the appropriate DRAS Clients in the RTP program. The event information includes the following:

- Utility event information for Intelligent DRAS Clients

o Date and time of the event

o Date and time issued

o Geographic location (optional)

o Mode and pending signals

- Mode signal levels for Simple DRAS Clients (e.g. normal, moderate, high)

- Event pending signal for Simple DRAS Clients (e.g. yes/no, or simple quantification of how far in advancee notice is to be sent)

3b. When configured, the RTP notifier in the DRAS updates the RTP information in the appropriate DRAS Clients. The RTP information includes the following:

- Utility RTP information for Intelligent DRAS Clients

o Date and time of the RTP

o sDate and time issued

o Geographic location (optional)

4. Based upon the price and/or event information received the DRAS Event Client for the participant site sends event information to all appropriate participant side 
systems which results in loads being shed or shifted. The details of this process are beyond the scope of this document.

5. The DRAS Feedback Client for the participant site sends the system load status to the DRAS. This is a feedback mechanism that is used to record how the participant site responded to the DR event. It includes the following information:

- Program identifier

- Facility identifier

- Date and time of the event (shed or shift)

- Shed data in $\mathrm{kW} / \mathrm{kWh}$

- Load reduction end uses (HVAC, lighting, etc.)

- Event Type (Day-Ahead or Day-Of)

6. The utility program settlement measures usage in the facility and performs settlement in the Utility Information System. The details of this process are beyond the scope of this document.

\section{D.2.7.3 RTP Maintenance}

This scenario consists of a set of actions which are necessary to maintain the RTP program. Unlike the configuration and execution scenarios this set of actions are less a prescribed set of steps and more a set of possible actions that may be performed by the various roles at unrelated times.

1a. The utility program operator gets the operation reports. The utility program operator can get the following status information from the DRAS at any time:

- Event status (for all participants)

o current outstanding events

- Load reduction potential based upon all customers in program (optional)

- Feedback from DRAS Clients (for those that have optional feedback)

o event logs

1b. The participant manager checks the status. The utility program operator can get the following status information from the DRAS at any time:

- DRAS Client communications status

o current status

o last contact

o current signals levels

o current customer manual control levels (opt-out)

o communication logs

o signal logs 
o manual control logs

- Event status (same as above)

1c. The utility program operator adds, modifies or deletes facilities participating in the program. This is similar to the original configuration step.

1d. The participant manager opts out of the DR program. At any time, the facility manager can opt out of the DR program on the DRAS. When there is an opt-out condition, DR events are not propagated to the DRAS Client. The opt-out can be either for the entire program or a single event.

1e. The participant manager modifies the RTP-event mapping logic.

1f. The utility program operator and/or the participant manager receive an exception notification from the DRAS in case of an error (this is not shown in the diagram). When an error condition occurs in the DRAS which may require some sort of action by either the participant or the utility the DRAS will send a message to the respective operators via email, voice or pager. Note that this alarming interface does not cover exception conditions that are part of the DRAS operation and managed by the DRAS operator, which is outside the scope of this document. The types of exceptions covered by this interface include:

- DRAS Client communications failure. 